\title{
Photoinduced Formation of Hybrid Aryl Pd-Radical Species Capable of 1,5-HAT: Selective Catalytic Oxidation of Silyl Ethers into Silyl Enol Ethers
}

\author{
Marvin Parasram, Padon Chuentragool, Dhruba Sarkar, and Vladimir Gevorgyan* \\ Department of Chemistry, University of Illinois at Chicago, 845 West Taylor Street, Chicago, Illinois 60607- \\ 7061, United States, vlad@uic.edu
}

Table of Contents

1. General Information 2

2. Synthesis of Starting Materials $\quad 3$

3. Optimization Tables 17

4. Oxidation of Silyl Ethers 19

5. Mechanistic Studies $\quad 31$

6. Stoichiometric Studies 36

7. NMR Spectral Data $\quad 39$

$\begin{array}{ll}\text { 8. } & \text { References } \\ & 104\end{array}$ 


\section{General Information}

NMR spectra were recorded on Bruker Avance DRX-500 (500 MHz) or DPX-400 (400 MHz)

instrument. ${ }^{1} \mathrm{H}$ signals are referenced to residual $\mathrm{CHCl}_{3}$ at $7.26 \mathrm{ppm} .{ }^{13} \mathrm{C}$ signals are referenced to $\mathrm{CDCl}_{3}$ at $77.0 \mathrm{ppm}$. GC/MS analysis was performed on a Hewlett Packard Model $6890 \mathrm{GC}$ interfaced to a Hewlett Packard Model 5973 mass selective detector (15 m x 0.25 mm capillary column, HP-5MS). Column chromatography was carried out employing Silicycle Silica-P flash silica gel $(40-63 \mu \mathrm{m})$. Precoated silica gel plates F-254 were used for thin-layer analytical chromatography. LRMS and HRMS analyses were performed on Micromass 70 VSE mass spectrometer. Anhydrous solvents purchased from Aldrich were additionally purified on PureSolv PS-400-4 by Innovative Technology, Inc. purification system and/or stored over calcium hydride. All starting materials were purchased from Strem Chemicals, Aldrich, Gelest Inc., TCI America, or Alfa Aesar, or synthesized via known literature procedures. The $34 \mathrm{~W}$ Blue LED lamp (Kessil KSH150B LED Grow Light), 23W Philips Household CFL, and Vornado 133 Small Air Circulator fan were purchased from amazon.com. All manipulations with transition metal catalysts were conducted in oven-dried glassware under inert atmosphere using a combination of glovebox and standard Schlenk techniques. 


\section{Synthesis of Starting Materials}

\section{Preparation of aryl chloro silane tether 1A:}

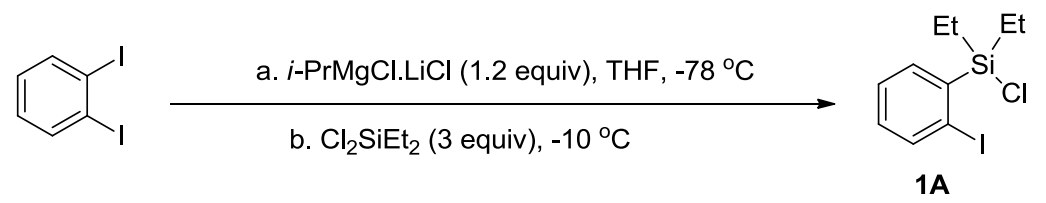

An oven dried $50 \mathrm{~mL}$ 2-neck flask connected with three ways gas adapter, containing a stirring bar, was charged with 1,2-diiodobenzene $(27 \mathrm{mmol})$. The flask was flushed with Argon three times, and then THF (35 mL) was added under Argon atmosphere. The reaction mixture was cooled to $-78{ }^{\circ} \mathrm{C}$ (dry ice and acetone bath), and turbo Grignard (1.3 $\mathrm{M}$ in THF, $32.4 \mathrm{mmol}$ ) was added to the reaction mixture. Reaction mixture was stirred at $-78{ }^{\circ} \mathrm{C}$ for $1 \mathrm{~h}$ until all 1,2 diiodobenzene was consumed (judged by GC/MS analysis). The resulting mixture was added diethyl dichlorosilane $(81 \mathrm{mmol})$ at $-78{ }^{\circ} \mathrm{C}$. Reaction mixture was stirred at $-10{ }^{\circ} \mathrm{C}$ for $3 \mathrm{~h}$ and then room temperature overnight. The excess dichlorosilane and THF were removed by vacuum for 3 hours and then $30 \mathrm{~mL}$ of dry hexanes were added under Argon atmosphere. The mixture was filtered under vacuum, washed with two portions of $10 \mathrm{~mL}$ hexanes, and left under vacuum for 3 more hours to remove hexanes. (78\% yields, $75-80 \%$ purity).

\section{Purification of aryl chloro silane tether 1A:}

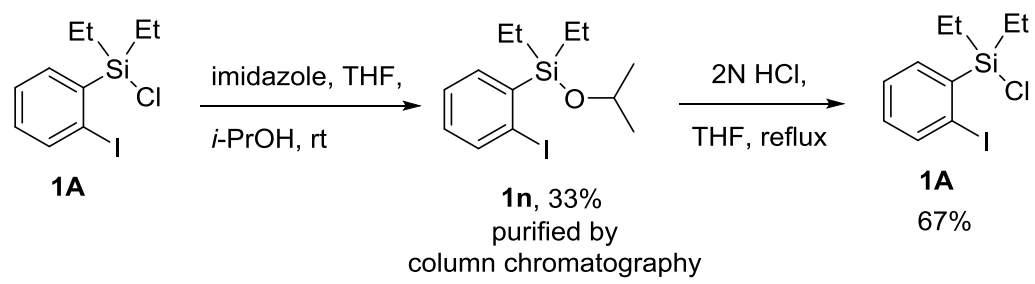

To a stirred mixture of imidazole (4.1g, $40 \mathrm{mmol}, 2$ equiv) and THF (60 mL), chlorosilane 1A (22 mmol, 1.1 equiv) was added at $\mathrm{rt}$ under argon atmosphere. To this mixture, isopropanol (20 mmol, 1 equiv) in $20 \mathrm{~mL}$ of THF was added. The mixture was stirred until completion of the reaction as judged by GC/MS. To this mixture, hexane $(30 \mathrm{~mL})$ was added and then filtered. The filtrate was then concentrated under reduced pressure. The residue was purified by column chromatography in 50:1 Hexanes: EtOAc to afford compound 1n.

To a solution of silylether 1 n $(2.5 \mathrm{mmol}, 1$ equiv $)$ in THF $(10 \mathrm{~mL}), 2 \mathrm{~N} \mathrm{HCl}$ in ether $(7.5 \mathrm{mmol}$, 3 equiv) was added at $\mathrm{rt}$ under argon atmosphere. The reaction mixture was refluxed until 
completion of the reaction as judged by GC/MS. The resulted solution was connected to vacuum for 30 minutes and then $10 \mathrm{~mL}$ of dry hexanes were added under Argon atmosphere. The mixture was filtered under vacuum, washed with two portions of $5 \mathrm{~mL}$ hexanes, and left under vacuum for 2 hours to remove hexanes. (67\% yields, $90 \%$ purity). ${ }^{1} \mathrm{H}$ NMR (500 MHz, $\left.\mathrm{CDCl}_{3}\right): \delta \mathrm{ppm} 7.88(\mathrm{~d}, J=8.0 \mathrm{~Hz}, 1 \mathrm{H}), 7.79(\mathrm{~d}, J=7.5 \mathrm{~Hz}, 1 \mathrm{H}), 7.40(\mathrm{t}, J=7.5 \mathrm{~Hz}, 1 \mathrm{H}), 7.10$ $(\mathrm{t}, J=8.0 \mathrm{~Hz}, 1 \mathrm{H}), 1.42-1.35(\mathrm{~m}, 2 \mathrm{H}), 1.25-1.19(\mathrm{~m}, 2 \mathrm{H}), 1.02(\mathrm{t}, J=8.0 \mathrm{~Hz} 6 \mathrm{H}) .{ }^{13} \mathrm{C}$ NMR $\left(126 \mathrm{MHz}, \mathrm{CDCl}_{3}\right) \delta \mathrm{ppm} 6.8,8.6,101.7,127.3,131.8,138.5,139.5,139.8$. LRMS expected for $\mathrm{C}_{10} \mathrm{H}_{24} \mathrm{IClSi}[\mathrm{M}]: 324.0$, found: 323.9 .

\section{General procedure for the preparation of silyl ethers 1a-y:}

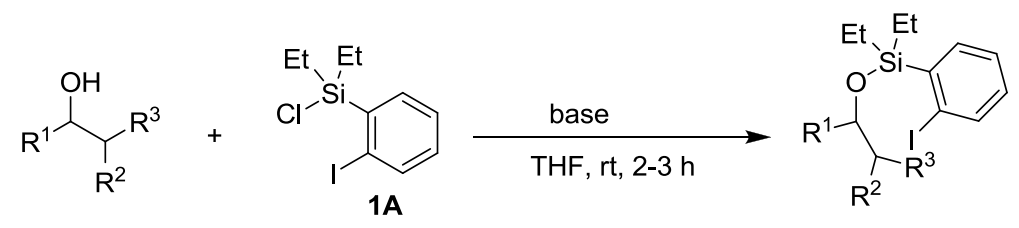

Procedure A: To a stirred mixture of imidazole ( $410 \mathrm{mg}, 4 \mathrm{mmol}, 2$ equiv) and THF (20 mL), chlorosilane 1A (2.4 mmol, 1.2 equiv) was added at rt under argon atmosphere. To this mixture, alcohol ( $2 \mathrm{mmol}, 1$ equiv) in $5 \mathrm{~mL}$ of THF was added. The mixture was stirred until completion of the reaction as judged by GC/MS. To this mixture, hexane $(10 \mathrm{~mL})$ was added and then filtered. The filtrate was then concentrated under reduced pressure. The residue was purified by column chromatography in 50:1 Hexanes: EtOAc.

Procedure B: To a stirred mixture of alcohol ( $2 \mathrm{mmol}, 1$ equiv) and THF (10 mL), MeLi (1.34 $\mathrm{mL}, 1.5 \mathrm{M}, 2 \mathrm{mmol}, 1$ equiv) was added dropwise at $0{ }^{\circ} \mathrm{C}$ under argon atmosphere. To this mixture, HMPA ( $0.35 \mathrm{~mL}, 2 \mathrm{mmol}, 1$ equiv) was added, followed by, chlorosilane 1A (2.2 mmol, 1.1 equiv) in $5 \mathrm{~mL}$ of THF was added at $0{ }^{\circ} \mathrm{C}$. The mixture was stirred until completion of the reaction by GC/MS. After completion the mixture was quenched with saturated ammonium chloride solution and extracted with DCM (3x $50 \mathrm{~mL})$. The combined organic layer was washed with brine. The organic layer was dried and filtered. The filtrate was then concentrated under reduced pressure. The residue was purified by column chromatography in 50:1 - 20:1 Hexanes: EtOAc. 


\section{Silyl Ether (1) Analytics:}

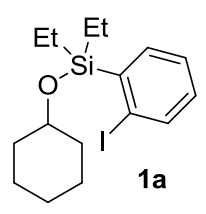

Was prepared according to general procedure $\mathbf{A}$ in $32 \%$ yield. Colorless oil. $\mathbf{R}_{\mathrm{f}}$ (hexanes): 0.31 . ${ }^{1} \mathrm{H}$ NMR (500 MHz, $\mathrm{CDCl}_{3}$ ): $\delta$ ppm 7.83-7.85 (m, 1H), 7.64-7.62 (m, 1H), 7.36-7.33 (m, 1H), 7.05-7.01 (m, 1H), 3.80-3.74 (m, 1H), 1.87-1.85 (m, 2H), 1.76-1.75 (m, 2H), 1.52-1.42 (m, $3 \mathrm{H}), 1.28-1.23(\mathrm{~m}, 3 \mathrm{H}), 1.14-1.0 .98(\mathrm{~m}, 4 \mathrm{H}), 0.96-0.93(\mathrm{~m}, 6 \mathrm{H}) .{ }^{13} \mathrm{C} \mathrm{NMR}\left(126 \mathrm{MHz}, \mathrm{CDCl}_{3}\right)$ $\delta$ ppm 5.5, 7.0, 24.2, 25.6, 35.9, 71.4, 102.7, 126.8, 130.9, 138.1, 139.6, 142.8. HRMS (EI+) calcd. for $\mathrm{C}_{16} \mathrm{H}_{25} \mathrm{IOSi}[\mathrm{M}]: 388.0720$, found: 388.0722 .

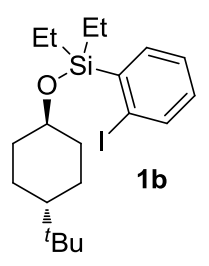

Was prepared according to general procedure $\mathbf{A}$ in $41 \%$ yield. Colorless oil. $\mathbf{R}_{\mathrm{f}}$ (hexanes): 0.35 . ${ }^{1} \mathrm{H}$ NMR (500 MHz, $\mathrm{CDCl}_{3}$ ): $\delta$ ppm 7.85-7.84 (m, 1H), 7.61-7.7.59 (m, 1H), 7.35-7.32 (m, 1H), 7.05-7.01 (m, 1H), 3.68-3.61 (m, 1H), 2.02-2.00 (m, 2H), 1.77-1.75 (m, 2H), 1.43-1.37 (m, 2H), 1.13-1.03 (m, 2H), 1.03-1.01 (m, 1H), 1.01-0.98 (m, 2H), 0.98-0.94 (m, 8H), 0.85$0.83(\mathrm{~m}, 9 \mathrm{H}) .{ }^{13} \mathrm{C} \mathrm{NMR}\left(126 \mathrm{MHz}, \mathrm{CDCl}_{3}\right) \delta \mathrm{ppm}$ 5.4, 7.0, 25.8, 27.6, 32.3, 36.4, 47.2, 72.6, 102.7, 126.8, 130.9, 138.1, 139.7, 142.8. HRMS (ESI) calcd. for $\mathrm{C}_{20} \mathrm{H}_{33} \mathrm{IOSi}[\mathrm{M}]+1$ : 444.1345, found: 444.1347.

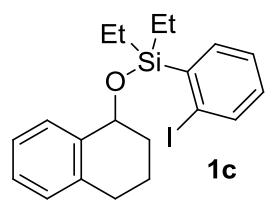

Was prepared according to general procedure $\mathbf{A}$ in $28 \%$ yield. Colorless oil. $\mathrm{R}_{\mathrm{f}}$ (hexanes:EtOAc = 20:1): 0.33. ${ }^{1} \mathrm{H}$ NMR $\left(500 \mathrm{MHz}, \mathrm{CDCl}_{3}\right): \delta \mathrm{ppm} 7.86(\mathrm{~d}, J=7.7 \mathrm{~Hz}, 1 \mathrm{H})$, $7.64(\mathrm{dd}, J=7.3 \mathrm{~Hz}, 1.8 \mathrm{~Hz}, 1 \mathrm{H}), 7.42-7.41$ (m, 1H), 7.33-7.31 (m, 1H), 7.20-7.17 (m, 2H), 7.11-7.10 (m, 1H), $7.05(\mathrm{dt}, J=7.7 \mathrm{~Hz}, 1.8 \mathrm{~Hz}, 1 \mathrm{H}), 5.00-4.98$ (m, 1H), 2.91-2.85 (m, 1H), 2.78-2.72 (m, 1H), 2.13-2.11 (m, 1H), 2.07-1.95 (m, 2H), 1.81-1.74 (m, 1H), 1.24-1.17 (m, 2H), 1.12-1.05 (m, 2H), 1.02-0.97 (m, 6H). $\left.{ }^{13} \mathrm{C} \mathrm{NMR} \mathrm{(126} \mathrm{MHz,} \mathrm{CDCl}_{3}\right): \delta \mathrm{ppm} 5.5,5.9,7.0$, 
7.1, 19.4, 29.0, 32.7, 69.8, 102.7, 125.7, 126.9, 127.1, 128.4, 128.8, 131.4, 137.0, 138.4, 139.3, 139.7, 142.4. HRMS (EI+) calcd. for $\mathrm{C}_{20} \mathrm{H}_{25} \mathrm{IOSi}[\mathrm{M}]: 436.0720$, found: 436.0726 .

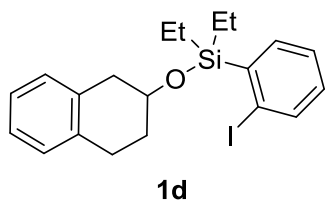

Was prepared according to general procedure $\mathbf{A}$ in $38 \%$ yield. Colorless oil. $\mathrm{R}_{\mathrm{f}}$ (hexanes:EtOAc $=20: 1): 0.33 .{ }^{1} \mathrm{H}$ NMR $\left(500 \mathrm{MHz}, \mathrm{CDCl}_{3}\right): \delta \mathrm{ppm} 7.86(\mathrm{~d}, J=8.0 \mathrm{~Hz}, 1 \mathrm{H})$, 7.59 (dd, $J=7.3 \mathrm{~Hz}, 1.8 \mathrm{~Hz}, 1 \mathrm{H}), 7.42-7.41(\mathrm{dt}, J=7.3 \mathrm{~Hz}, 1.8 \mathrm{~Hz}, 1 \mathrm{H}), 7.13-7.03(\mathrm{~m}, 5 \mathrm{H})$, 4.28-4.23 (m, 1H), 3.09-2.98 (m, 2H), 2.94-2.89 (m, 1H), 2.86-2.80 (m, 1H), 2.11-2.07 (m, $1 \mathrm{H}), 1.96-1.90(\mathrm{~m}, 1 \mathrm{H}), 1.19-1.12(\mathrm{~m}, 2 \mathrm{H}), 1.08-1.02(\mathrm{~m}, 2 \mathrm{H}), 0.99-0.97(\mathrm{~m}, 6 \mathrm{H}) .{ }^{13} \mathrm{C}$ NMR (126 MHz, $\left.\mathrm{CDCl}_{3}\right): \delta$ ppm 5.4, 5.9, 7.0, 7.1, 27.6, 32.1, 39.0, 68.6, 102.8, 125.7, 125.8, 126.9, $128.5,129.4,131.4,131.0,135.0,135.9,138.0,139.7,142.4$. HRMS (CI) calcd. for $\mathrm{C}_{20} \mathrm{H}_{25} \mathrm{IOSi}[\mathrm{M}]-1: 435.0642$, found: 435.0639 .

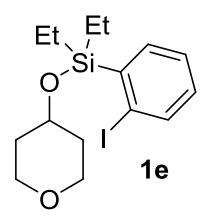

Was prepared according to general procedure $\mathbf{A}$ in $46 \%$ yield. Colorless oil. $\mathrm{R}_{\mathrm{f}}$ (hexanes): 0.08 . ${ }^{1} \mathrm{H}$ NMR (500 MHz, $\mathrm{CDCl}_{3}$ ): $\delta \mathrm{ppm} 7.85(\mathrm{~d}, J=8.0 \mathrm{~Hz}, 1 \mathrm{H}), 7.59$ (d, $\left.J=7.5 \mathrm{~Hz}, 1 \mathrm{H}\right), 7.35$ (t, $J$ $=7.5 \mathrm{~Hz}, 1 \mathrm{H}), 7.06(\mathrm{t}, J=8.0 \mathrm{~Hz}, 1 \mathrm{H}), 4.01-3.95(\mathrm{~m}, 3 \mathrm{H}), 3.50-3.45(\mathrm{~m}, 2 \mathrm{H}), 1.88-1.85(\mathrm{~m}$, $2 \mathrm{H}), 1.71-1.68(\mathrm{~m}, 2 \mathrm{H}), 1.12-1.10(\mathrm{~m}, 2 \mathrm{H}), 1.02-0.93(\mathrm{~m}, 8 \mathrm{H}) .{ }^{13} \mathrm{C} \mathrm{NMR}\left(126 \mathrm{MHz}, \mathrm{CDCl}_{3}\right) \delta$ ppm 5.4, 7.0, 35.7, 65.5, 67.7, 102.6, 126.9, 131.1, 137.9, 139.8, 142.2 HRMS (AP+) calcd. for $\mathrm{C}_{15} \mathrm{H}_{25} \mathrm{IO}_{2} \mathrm{Si}[\mathrm{M}]+1: 391.0590$, found: 391.0592 .

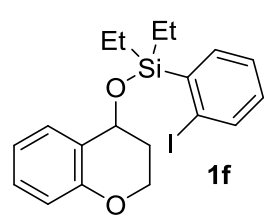

Was prepared according to general procedure $\mathbf{A}$ in $38 \%$ yield. Colorless oil. $\mathrm{R}_{\mathrm{f}}$ (hexanes:EtOAc = 20:1): 0.31. ${ }^{1} \mathrm{H}$ NMR $\left(500 \mathrm{MHz}, \mathrm{CDCl}_{3}\right): \delta \mathrm{ppm}$ 7.87-7.85 $(\mathrm{m}, 1 \mathrm{H}), 7.56$ (dd, $J=7.7 \mathrm{~Hz}, 1.8 \mathrm{~Hz}, 1 \mathrm{H}), 7.34-7.31(\mathrm{~m}, 1 \mathrm{H}), 7.19-7.16(\mathrm{~m}, 2 \mathrm{H}), 7.01-7.04(\mathrm{~m}, 1 \mathrm{H}), 6.85-$ 
$6.82(\mathrm{~m}, 2 \mathrm{H}), 4.95(\mathrm{t}, J=4.3 \mathrm{~Hz}, 1 \mathrm{H}), 4.50-4.46(\mathrm{~m}, 1 \mathrm{H}), 4.30-4.26(\mathrm{~m}, 1 \mathrm{H}), 2.11-2.08(\mathrm{~m}$, 1H), 1.22-1.04 (m, 4H), 1.02-0.97 (m, 6H). ${ }^{13} \mathrm{C}$ NMR (126 MHz, $\left.\mathrm{CDCl}_{3}\right) \delta$ ppm 5.4, 5.7, 6.9, 31.6, 64.3, 64.6, 32.7, 69.8, 102.7, 116.7, 119.9, 124.3, 126.9, 129.3, 129.9, 131.2, 138.1, 139.8, 139.7, 142.0. HRMS (ESI) calcd. for $\mathrm{C}_{19} \mathrm{H}_{23} \mathrm{IO}_{2} \mathrm{Si}[\mathrm{M}]+1$ : 438.0512, found: 438.0514 .

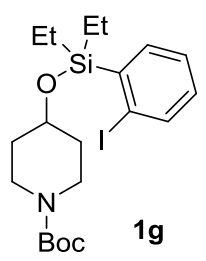

Was prepared according to general procedure $A$ in $43 \%$ yield. Colorless oil. $\mathrm{R}_{\mathrm{f}}$ (hexanes:EtOAc = 20:1): 0.23. ${ }^{1} \mathrm{H}$ NMR $\left(500 \mathrm{MHz}, \mathrm{CDCl}_{3}\right): \delta \mathrm{ppm} 7.85(\mathrm{~d}, J=7.7 \mathrm{~Hz}, 1 \mathrm{H})$, $7.57(\mathrm{dd}, J=7.7 \mathrm{~Hz}, 1.8 \mathrm{~Hz}, 1 \mathrm{H}), 7.34(\mathrm{t}, J=7.3 \mathrm{~Hz}, 1 \mathrm{H}), 7.05(\mathrm{dt}, J=8.0 \mathrm{~Hz}, 1.4 \mathrm{~Hz}, 1 \mathrm{H})$, 4.02-3.98 (m, 1H), 3.74 (br. s, 2H), 3.24-3.19 (m, 2H), 1.78 (br. s, 2H), 1.61 (br. s, $2 \mathrm{H}$ ), 1.46 (s, 9H), 1.13-1.08 (m, 2H), 1.02-0.98 (m, 2H), 0.96-0.93 (m, 6H). ${ }^{13} \mathrm{C}$ NMR (126 MHz, $\left.\mathrm{CDCl}_{3}\right) \delta$ ppm 5.3, 6.9, 28.5, 34.3, 68.2, 79.3, 102.6, 126.9, 131.1, 137.9, 139.8, 142.1, 145.9. HRMS (ESI) for $\mathrm{C}_{20} \mathrm{H}_{32} \mathrm{INO}_{3} \mathrm{Si}[\mathrm{M}]+1: 490.1263$, found: 490.1263 .

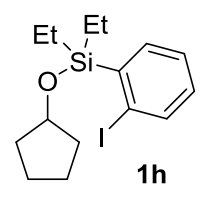

Was prepared according to general procedure $\mathbf{A}$ in $31 \%$ yield. Colorless oil. $\mathbf{R}_{\mathbf{f}}$ (hexanes): 0.27 . ${ }^{1} \mathrm{H}$ NMR $\left(500 \mathrm{MHz}, \mathrm{CDCl}_{3}\right): \delta \mathrm{ppm} 7.83-7.82(\mathrm{~d}, J=7.89 \mathrm{~Hz}, 1 \mathrm{H}), 7.59-7.55(\mathrm{dd}, J=7.31 \mathrm{~Hz}$, $J=2.05 \mathrm{~Hz}, 1 \mathrm{H}), 7.36-7.31(\mathrm{td},, J=7.31 \mathrm{~Hz}, J=1.17 \mathrm{~Hz}, 1 \mathrm{H}), 7.05-7.00(\mathrm{td},, J=7.60 \mathrm{~Hz}, J$ $=2.05 \mathrm{~Hz}, 1 \mathrm{H}), 4.38-4.36(\mathrm{~m}, 1 \mathrm{H}), 1.79-1.68(\mathrm{~m}, 6 \mathrm{H}), 1.55-1.53(\mathrm{~m}, 2 \mathrm{H}), 1.12-0.98(\mathrm{~m}, 4 \mathrm{H})$, 0.96-0.92 (m, 6H). ${ }^{13} \mathrm{C}$ NMR (126 MHz, $\left.\mathrm{CDCl}_{3}\right) \delta \mathrm{ppm} 5.3,6.9,23.2,35.7,74.9,102.6,126.8$, 130.9, 138.0, 139.7, 142.7. HRMS (EI+) calcd. for $\mathrm{C}_{15} \mathrm{H}_{23} \mathrm{IOSi}[\mathrm{M}]$ : 374.0563, found: 374.0578 . 


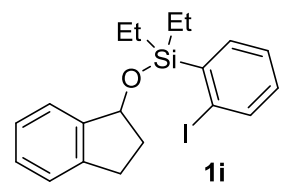

Was prepared according to general procedure $\mathbf{A}$ in $32 \%$ yield. Colorless oil. $\mathrm{R}_{\mathrm{f}}$ (hexanes:EtOAc = 20:1): 0.33. ${ }^{1} \mathrm{H}$ NMR $\left(500 \mathrm{MHz}, \mathrm{CDCl}_{3}\right): \delta \mathrm{ppm} 7.87(\mathrm{~m}, 1 \mathrm{H}), 7.66(\mathrm{dd}, J=$ 7.3 Hz, 1.8 Hz, 1H), 7.43-7.41 (m, 1H), 7.36-7.33 (m, 1H), 7.26-7.22 (m, 3H), 7.06 (dt, $J=7.7$ $\mathrm{Hz}, 1.8 \mathrm{~Hz}, 1 \mathrm{H}), 5.41(\mathrm{t}, J=6.0 \mathrm{~Hz}, 1 \mathrm{H}), 3.07-3.03(\mathrm{~m}, 1 \mathrm{H}), 2.83-2.77(\mathrm{~m}, 1 \mathrm{H}), 2.46-2.40(\mathrm{~m}$, $1 \mathrm{H}), 2.14-2.06(\mathrm{~m}, 2 \mathrm{H}), 1.27-1.18(\mathrm{~m}, 1 \mathrm{H}), 1.15-1.08(\mathrm{~m}, 2 \mathrm{H}), 1.04-0.99(\mathrm{~m}, 6 \mathrm{H}) .{ }^{13} \mathrm{C}$ NMR $\left(126 \mathrm{MHz}, \mathrm{CDCl}_{3}\right): \delta$ ppm 5.4, 5.7, 6.9, 7.0, 29.9, 36.5, 76.9, 102.7, 124.4, 124.7, 126.4, 126.9, $127.8,131.1,138.3,139.7,142.2,142.8,145.3$. HRMS (EI+) calcd. for $\mathrm{C}_{19} \mathrm{H}_{23} \mathrm{IOSi}[\mathrm{M}]$ : 422.0563, found: 422.0554 .

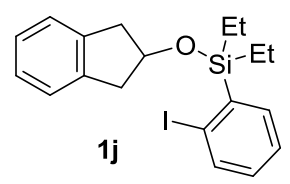

Was prepared according to general procedure $\mathbf{A}$ in $33 \%$ yield. Colorless oil. $\mathrm{R}_{\mathrm{f}}$ (hexanes:EtOAc $=20: 1): 0.33 .{ }^{1} \mathrm{H}$ NMR $\left(500 \mathrm{MHz}, \mathrm{CDCl}_{3}\right): \delta \mathrm{ppm}$ 7.87-7.85 $(\mathrm{m}, 1 \mathrm{H}), 7.55$ (dd, $J=7.3 \mathrm{~Hz}, 1.8 \mathrm{~Hz}, 1 \mathrm{H}), 7.35-7.32(\mathrm{~m}, 1 \mathrm{H}), 7.21-7.15(\mathrm{~m}, 4 \mathrm{H}), 7.05(\mathrm{dt}, J=8.1 \mathrm{~Hz}, 1.8$ $\mathrm{Hz}, 1 \mathrm{H}$ ), 4.79 (quintet, $J=6.2 \mathrm{~Hz}, 1 \mathrm{H}), 3.21-3.17(\mathrm{~m}, 2 \mathrm{H}), 3.08-3.03(\mathrm{~m}, 2 \mathrm{H}), 1.17-1.13(\mathrm{~m}$, $4 \mathrm{H}), 1.00-0.97(\mathrm{~m}, 6 \mathrm{H}) .{ }^{13} \mathrm{C}$ NMR $\left(126 \mathrm{MHz}, \mathrm{CDCl}_{3}\right): \delta \mathrm{ppm} 5.2,6.9,42.4,74.3,102.6,124.6$, $126.5,126.9,131.1,138.0,139.8,141.0,142.1$. HRMS (EI+) calcd. for $\mathrm{C}_{19} \mathrm{H}_{23} \mathrm{IOSi}[\mathrm{M}]$ : 422.0563, found: 422.0566 .

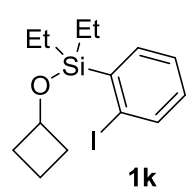

Was prepared according to general procedure $\mathbf{A}$ in $36 \%$ yield. Colorless oil. $\mathrm{R}_{\mathrm{f}}$ (hexanes): 0.12 . ${ }^{1} \mathrm{H}$ NMR (500 MHz, $\mathrm{CDCl}_{3}$ ): $\delta$ ppm 7.85-7.83 (d, $\left.J=8.07 \mathrm{~Hz}, 1 \mathrm{H}\right), 7.54-7.53(\mathrm{dd}, J=7.34 \mathrm{~Hz}$, $J=1.47 \mathrm{~Hz}, 1 \mathrm{H}), 7.36-7.33(\mathrm{t}, 1 \mathrm{H}), 7.05-7.02(\mathrm{td}, J=7.70 \mathrm{~Hz}, J=1.10 \mathrm{~Hz}, 1 \mathrm{H}), 4.32-4.26(\mathrm{~m}$, $1 \mathrm{H}), 2.26-2.21(\mathrm{~m}, 2 \mathrm{H}), 2.11-2.03(\mathrm{~m}, 2 \mathrm{H}), 1.67-1.61(\mathrm{q}, 1 \mathrm{H}), 1.43-1.37(\mathrm{~m}, 1), 1.07-0.99(\mathrm{~m}$, $4 \mathrm{H}), 0.98-0.93(\mathrm{~m}, 6 \mathrm{H}) .{ }^{13} \mathrm{C}$ NMR $\left(126 \mathrm{MHz}, \mathrm{CDCl}_{3}\right) \delta \mathrm{ppm} 5.2,6.8,12.3,33.8,67.4,102.5$, 
126.6, 131.0, 137.8, 139.6, 142.4. HRMS (AP+) calcd. for $\mathrm{C}_{14} \mathrm{H}_{22} \mathrm{IOSi}[\mathrm{M}]+1: 361.0485$, found: 361.0493 .

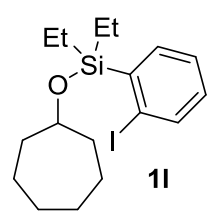

Was prepared according to general procedure $\mathbf{A}$ in $29 \%$ yield. Colorless oil. $\mathrm{R}_{\mathrm{f}}$ (hexanes): 0.29 . ${ }^{1} \mathrm{H}$ NMR $\left(500 \mathrm{MHz}, \mathrm{CDCl}_{3}\right): \delta \mathrm{ppm} 7.85-7.83(\mathrm{~d}, J=7.89 \mathrm{~Hz}, 1 \mathrm{H}), 7.64-7.63(\mathrm{dd}, J=7.60 \mathrm{~Hz}$, $J=2.05 \mathrm{~Hz}, 1 \mathrm{H}), 7.36-7.31(\mathrm{td},, J=7.60 \mathrm{~Hz}, J=1.17 \mathrm{~Hz}, 1 \mathrm{H}), 7.04-7.01(\mathrm{td},, J=7.89 \mathrm{~Hz}, J$ $=2.05 \mathrm{~Hz}, 1 \mathrm{H}), 4.02-3.98(\mathrm{~m}, 1 \mathrm{H}), 1.90-1.85(\mathrm{~m}, 2 \mathrm{H}), 1.76-1.67(\mathrm{~m}, 4 \mathrm{H}), 1.59-1.52(\mathrm{~m}, 4 \mathrm{H})$, 1.41-1.35 (m, 2H), 1.14-1.07 (m, 2H), 1.02-0.92 (m, 8H). $\left.{ }^{13} \mathrm{C} \mathrm{NMR} \mathrm{(125} \mathrm{MHz,} \mathrm{CDCl}_{3}\right) \delta \mathrm{ppm}$ 5.4, 5.9, 7.0, 23.6, 28.2, 37.8, 73.7, 109.6, 126.8, 130.9, 138.1, 139.6, 146.9. HRMS (EI+) calcd. for $\mathrm{C}_{17} \mathrm{H}_{27} \mathrm{IOSi}[\mathrm{M}]:: 402.0876$ found: 402.0877.

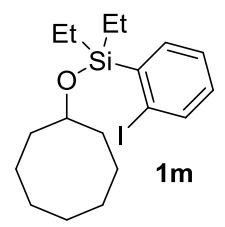

Was prepared according to general procedure $\mathbf{A}$ in $48 \%$ yield. Colorless oil. $\mathrm{R}_{\mathrm{f}}$ (hexanes): 0.29. ${ }^{1} \mathrm{H}$ NMR (500 MHz, $\mathrm{CDCl}_{3}$ ): $\delta \mathrm{ppm} 7.85-7.83(\mathrm{~d}, J=7.89 \mathrm{~Hz}, 1 \mathrm{H}), 7.64-7.62(\mathrm{dd}, J=7.60 \mathrm{~Hz}$, $J=1.75 \mathrm{~Hz}, 1 \mathrm{H}) 7.36-7.32(\mathrm{td}, J=7.31 \mathrm{~Hz}, J=1.17 \mathrm{~Hz}, 1 \mathrm{H}), 7.05-7.01(\mathrm{td}, J=7.60 \mathrm{~Hz}, J=$ $2.05 \mathrm{~Hz}, 1 \mathrm{H}), 4.01-3.97(\mathrm{~m}, 1 \mathrm{H}), 1.82-1.78(\mathrm{~m}, 6 \mathrm{H}), 1.54-1.45(\mathrm{~m}, 9 \mathrm{H}), 1.56-1.53(\mathrm{~m}, 5 \mathrm{H})$, 1.12-0.98 (m, 4H), 0.96-0.92 (m, 6H). ${ }^{13} \mathrm{C}$ NMR (126 MHz, $\left.\mathrm{CDCl}_{3}\right) \delta \mathrm{ppm} \mathrm{5.4,} \mathrm{7.0,} \mathrm{22.6,} \mathrm{25.6,}$ 27.6, 34.8, 73.1, 102.7, 126.8, 130.9, 138.1, 139.6, 142.8. HRMS (EI+) calcd. for $\mathrm{C}_{18} \mathrm{H}_{29} \mathrm{IOSi}$ [M]+1: 416.1032, found: 416.1037 .

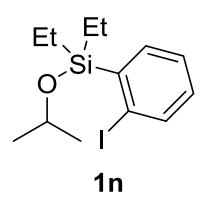

Was prepared according to general procedure $\mathbf{A}$ in $33 \%$ yield. Colorless oil. $\mathbf{R}_{\mathrm{f}}$ (50:1-Hexanes: EtOAc): 0.10. ${ }^{1} \mathrm{H}$ NMR $\left(500 \mathrm{MHz}, \mathrm{CDCl}_{3}\right): \delta \mathrm{ppm} 7.86-7.84(\mathrm{~d}, J=8.07 \mathrm{~Hz}, 1 \mathrm{H}), 7.62-7.60$ $(\mathrm{dd}, J=7.34 \mathrm{~Hz}, J=1.47 \mathrm{~Hz}, 1 \mathrm{H}), 7.36-7.32(\mathrm{td}, J=7.34 \mathrm{~Hz}, J=1.10 \mathrm{~Hz}, 1 \mathrm{H}), 7.05-7.02(\mathrm{td}$, $J=7.70 \mathrm{~Hz}, J=1.83 \mathrm{~Hz}, 1 \mathrm{H}), 4.17-4.12(\mathrm{~m}, 1 \mathrm{H}), 1.26-1.23(\mathrm{~d}, J=6.24,6 \mathrm{H}), 1.14-1.01(\mathrm{~m}$, 
4H), 0.99-0.95 (m, 6H). ${ }^{13} \mathrm{C}$ NMR (126 MHz, $\left.\mathrm{CDCl}_{3}\right) \delta \mathrm{ppm} \mathrm{5.3,} \mathrm{6.9,} \mathrm{25.9,} \mathrm{65.5,} \mathrm{102.7,} \mathrm{126.8,}$ 130.9, 138.1, 139.7, 142.6. LRMS expected for $\mathrm{C}_{13} \mathrm{H}_{21} \mathrm{IOSi}=348.3$, found: 347.9.

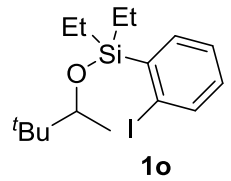

Was prepared according to general procedure $\mathbf{A}$ in $50 \%$ yield. Colorless oil. $\mathbf{R}_{\mathrm{f}}$ (hexanes): 0.36. ${ }^{1} \mathrm{H}$ NMR (400 MHz, $\left.\mathrm{CDCl}_{3}\right): \delta \mathrm{ppm} 7.86(\mathrm{~d}, J=8.0 \mathrm{~Hz}, 1 \mathrm{H}), 7.70(\mathrm{~d}, J=7.5 \mathrm{~Hz}, 1 \mathrm{H}), 7.36(\mathrm{t}, J$ $=7.5 \mathrm{~Hz}, 1 \mathrm{H}), 7.04(\mathrm{t}, J=8.0 \mathrm{~Hz}, 1 \mathrm{H}), 3.70(\mathrm{q}, J=6.7 \mathrm{~Hz}, 1 \mathrm{H}), 1.14(\mathrm{~d}, J=6.7 \mathrm{~Hz}, 3 \mathrm{H})$, 1.00-0.87 (m, 19H). ${ }^{13} \mathrm{C}$ NMR (100 MHz, $\left.\mathrm{CDCl}_{3}\right) \delta$ ppm 5.5, 7.1, 18.4, 26.0, 35.7, 76.9, 102.7, 126.4, 130.8, 138.2, 139.7, 143.2. HRMS (EI+) calcd. for $\mathrm{C}_{16} \mathrm{H}_{27} \mathrm{IOSi}$ [M]: 390.0976, found: 390.0862 .

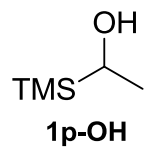

An oven dried $25 \mathrm{~mL}$ round bottom flask containing a stirring bar was charged with lithium aluminum hydride ( $7.5 \mathrm{mmol}, 1.5$ equiv). The flask was cap with a septum and the reaction was flashed with argon balloons (2 times). The flask was then cooled to $0{ }^{\circ} \mathrm{C}$ and $10 \mathrm{~mL}$ of diethyl ether was added. After that, acetyltrimethylsilane (5 mmol, 1 equiv) was added (dropwise) to the reaction mixture. The reaction was stirred for 2 hours at $0{ }^{\circ} \mathrm{C}$, then $10 \mathrm{~mL}$ of $\mathrm{NH}_{4} \mathrm{Cl}$ was added. The mixture was extracted with DCM, dried with $\mathrm{Na}_{2} \mathrm{SO}_{4}$ and filtered, concentrated, and then used for the next step without further purification.

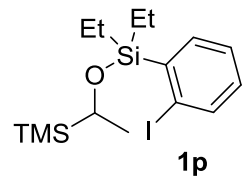

Was prepared according to general procedure A in 29\% yield using alcohol 1p-OH. Colorless oil. $\mathrm{R}_{\mathrm{f}}$ (hexanes): $0.34 .{ }^{1} \mathrm{H}$ NMR $\left(500 \mathrm{MHz}, \mathrm{CDCl}_{3}\right): \delta \mathrm{ppm} 7.83(\mathrm{~d}, J=8.0 \mathrm{~Hz}, 1 \mathrm{H}), 7.64(\mathrm{~d}, J$ $=7.5 \mathrm{~Hz}, 1 \mathrm{H}), 7.33(\mathrm{t}, J=7.5 \mathrm{~Hz}, 1 \mathrm{H}), 7.02(\mathrm{t}, J=8.0 \mathrm{~Hz}, 1 \mathrm{H}), 3.70(\mathrm{q}, J=7.5 \mathrm{~Hz}, 1 \mathrm{H}), 1.26$ $(\mathrm{d}, J=7.5 \mathrm{~Hz}, 3 \mathrm{H}), 1.15-1.05(\mathrm{~m}, 2 \mathrm{H}) 0.98-0.90(\mathrm{~m}, 8 \mathrm{H}), 0.06(\mathrm{~s}, 9 \mathrm{H}) .{ }^{13} \mathrm{C} \mathrm{NMR}(126 \mathrm{MHz}$, $\left.\mathrm{CDCl}_{3}\right) \delta$ ppm 3.9, 5.5, 5.7, 7.1, 19.3, 62.8, 102.6, 126.7, 130.8, 138.1, 139.5, 138.1, 139.5, 143.3 HRMS (AP+) calcd. for $\mathrm{C}_{15} \mathrm{H}_{27} \mathrm{IOSi}_{2}[\mathrm{M}]+1$ : 407.0723, found: 407.0731. 


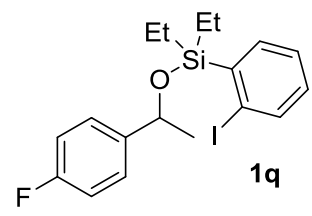

Was prepared according to general procedure $\mathbf{A}$ in $29 \%$ yield. Colorless oil. $\mathrm{R}_{\mathrm{f}}(50: 1$-Hexanes: EtOAc): 0.10. ${ }^{1} \mathrm{H}$ NMR $\left(500 \mathrm{MHz}, \mathrm{CDCl}_{3}\right): \delta \mathrm{ppm} 7.86-7.84(\mathrm{~d}, J=7.89,1 \mathrm{H}), 7.60-7.58(\mathrm{dd}, J$ $=7.60 \mathrm{~Hz}, J=2.05 \mathrm{~Hz}, 1 \mathrm{H}), 7.35-7.32(\mathrm{~m}, 3 \mathrm{H}), 7.07-6.98(\mathrm{~m}, 3 \mathrm{H}), 4.99-4.95(\mathrm{~m}, 1 \mathrm{H}), 1.52-$ $1.48(\mathrm{~d}, J=6.43,3 \mathrm{H}), 1.08-0.80(\mathrm{~m}, 10 \mathrm{H}) .{ }^{13} \mathrm{C}$ NMR $\left(126 \mathrm{MHz}, \mathrm{CDCl}_{3}\right) \delta \mathrm{ppm} 5.2,5.4,6.8$, 6.9, 27.2, 70.9, 102.6, 114.8, 115.0, 126.9, 127.0, 127.1, 131.1, 137.9, 139.7, 142.1, 160.9, 162.8,. HRMS (AP+) calcd. for $\mathrm{C}_{18} \mathrm{H}_{21}$ FIOSi [M]-1: 427.0390, found: 427.0396 .

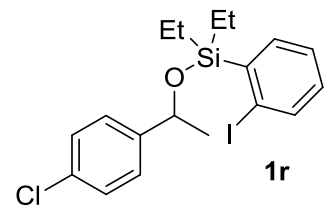

Was prepared according to general procedure $\mathbf{A}$ in $30 \%$ yield. Colorless oil. $\mathrm{R}_{\mathrm{f}}$ (hexanes): 0.15 . ${ }^{1} \mathrm{H}$ NMR $\left(500 \mathrm{MHz}, \mathrm{CDCl}_{3}\right): \delta \mathrm{ppm} 7.88-7.85(\mathrm{dd}, J=7.89 \mathrm{~Hz}, J=0.58 \mathrm{~Hz}, 1 \mathrm{H}), 7.62-7.60$ $(\mathrm{dd}, J=7.89 \mathrm{~Hz}, J=2.05 \mathrm{~Hz}, 1 \mathrm{H}), 7.37-7.29(\mathrm{~m}, 5 \mathrm{H}), 7.08-7.04(\mathrm{td}, J=7.31 \mathrm{~Hz}, J=1.75 \mathrm{~Hz}$, $1 \mathrm{H}), 5.01-4.96(\mathrm{q}, 1 \mathrm{H}), 1.53-1.50(\mathrm{~d}, J=6.14,3 \mathrm{H}), 1.10-0.82(\mathrm{~m}, 10 \mathrm{H}) .{ }^{13} \mathrm{C}$ NMR $(126 \mathrm{MHz}$, $\left.\mathrm{CDCl}_{3}\right) \delta \mathrm{ppm} 5.2,5.4,6.8,6.9,27.2,70.9,102.6,126.9,128.4,131.1,137.9,139.7,142.1$, 146.9. HRMS (AP+) calcd. for $\mathrm{C}_{18} \mathrm{H}_{21} \mathrm{ClIOSi}[\mathrm{M}]-1: 433.0095$, found: 433.0091 .

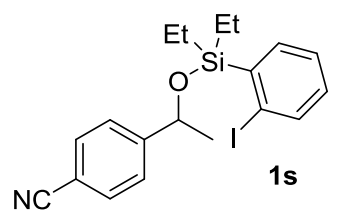

Was prepared according to general procedure $\mathbf{A}$ in $6 \%$ yield. Colorless oil. $\mathrm{R}_{\mathrm{f}}(20: 1$-Hexanes: EtOAc): 0.12. ${ }^{1} \mathrm{H}$ NMR $\left(500 \mathrm{MHz}, \mathrm{CDCl}_{3}\right): \delta \mathrm{ppm} 7.86-7.84(\mathrm{~d}, J=7.70 \mathrm{~Hz}, 1 \mathrm{H}), 7.62-7.61$ $(\mathrm{d}, J=8.44 \mathrm{~Hz}, 2 \mathrm{H}), 7.57-7.55(\mathrm{dd}, J=7.34 \mathrm{~Hz}, J=1.83 \mathrm{~Hz}, 1 \mathrm{H}), 7.50-7.48(\mathrm{~d}, J=8.44 \mathrm{~Hz}$, $2 \mathrm{H}), 7.36-7.33(\mathrm{td}, J=7.34 \mathrm{~Hz}, J=0.73 \mathrm{~Hz}, 1 \mathrm{H}), 7.08-7.04(\mathrm{td}, 7.34 \mathrm{~Hz}, J=1.47 \mathrm{~Hz}, 1 \mathrm{H})$, 5.02-5.01 (q, 1H), 1.50-1.49 (d, $6.60 \mathrm{~Hz}, 3 \mathrm{H}), 1.09-0.82(\mathrm{~m}, 10 \mathrm{H}) .{ }^{13} \mathrm{C}$ NMR $(126 \mathrm{MHz}$, $\left.\mathrm{CDCl}_{3}\right) \delta$ ppm 5.1, 5.4, 6.8, 6.9, 26.9, 70.9, 102.6, 118.9, 126.2, 126.9, 131.3, 132.2, 137.8, 139.9, 141.6, 151.7. HRMS (AP+) calcd. for $\mathrm{C}_{19} \mathrm{H}_{23} \mathrm{INOSi}[\mathrm{M}]+1$ : 436.0594, found: 436.0610 . 


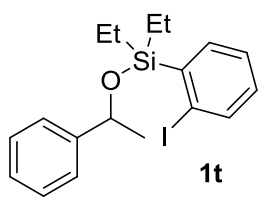

Was prepared according to general procedure $\mathbf{A}$ in 35\% yield. Colorless oil. $\mathrm{R}_{\mathrm{f}}$ (hexanes): 0.29. ${ }^{1} \mathrm{H}$ NMR $\left(500 \mathrm{MHz}, \mathrm{CDCl}_{3}\right): \delta \mathrm{ppm} 7.85(\mathrm{~d}, J=8.0 \mathrm{~Hz}, 1 \mathrm{H}), 7.63(\mathrm{~d}, J=7.5 \mathrm{~Hz}, 1 \mathrm{H}), 7.39(\mathrm{~m}$, 2H), $7.33(\mathrm{~m}, 3 \mathrm{H}), 7.25(\mathrm{~m}, 1 \mathrm{H}), 7.04(\mathrm{t}, J=8.0 \mathrm{~Hz}, 1 \mathrm{H}), 4.99(\mathrm{q}, J=6.0 \mathrm{~Hz}, 1 \mathrm{H}), 1.54(\mathrm{~d}, J=$ $6.0 \mathrm{~Hz}, 3 \mathrm{H}), 1.07-1.01$ (m, 2H) 0.94-0.88 (m, 5H), 0.83-0.77 (m, 3H). ${ }^{13} \mathrm{C}$ NMR (126 MHz, $\left.\mathrm{CDCl}_{3}\right) \delta \mathrm{ppm} 5.2,6.8,27.2,71.6,102.6,125.5,126.9,127.0,127.7,128.2,131.0,134.1$, 138.1, 139.7, 142.3, 146.4 HRMS (EI+) calcd. for $\mathrm{C}_{18} \mathrm{H}_{23} \mathrm{IOSi}[\mathrm{M}]$ : 410.0563, found: 410.0557.

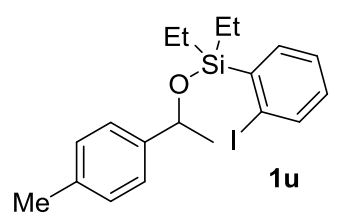

Was prepared according to general procedure $\mathbf{A}$ in $70 \%$ yield. Colorless oil. $\mathrm{R}_{\mathrm{f}}$ (hexanes): 0.3 . ${ }^{1} \mathrm{H}$ NMR $\left(500 \mathrm{MHz}, \mathrm{CDCl}_{3}\right): \delta \mathrm{ppm} 7.86-7.88(\mathrm{~d}, J=7.70 \mathrm{~Hz}, 1 \mathrm{H}), 7.66-7.64(\mathrm{dd}, J=7.34$ $\mathrm{Hz}, J=1.83 \mathrm{~Hz}, 1 \mathrm{H}), 7.36-7.35$ (t, 1H), 7.32-7.20 (d, $J=7.70 \mathrm{~Hz}, 2 \mathrm{H}), 7.17-7.15$ (d, $J=7.70$ $\mathrm{Hz}, 2 \mathrm{H}), 7.03-7.07$ (td, $J=7.70 \mathrm{~Hz}, J=1.83 \mathrm{~Hz}, 2 \mathrm{H}), 5.00-4.96(\mathrm{q}, 1 \mathrm{H}), 2.36(\mathrm{~s}, 3 \mathrm{H}), 1.52-$ $1.54(\mathrm{~d}, 6.24 \mathrm{~Hz}, 3 \mathrm{H}), 1.05-1.03(\mathrm{~m}, 2 \mathrm{H}), 0.96-0.91(\mathrm{~m}, 5 \mathrm{H}), 0.83-0.80$ (m, 3H). ${ }^{13} \mathrm{C}$ NMR $(126$ $\left.\mathrm{MHz}, \mathrm{CDCl}_{3}\right) \delta \mathrm{ppm} 5.3,5.5,6.8,7.0,21.2,27.1,71.4,102.6,125.2,125.5,126.8,128.9$, 131.0, 138.2, 139.7, 142.4, 143.4. HRMS (EI+) calcd. for $\mathrm{C}_{19} \mathrm{H}_{25} \mathrm{IO}_{2} \mathrm{Si}[\mathrm{M}]$ : 424.0720:, found: 424.0705 .

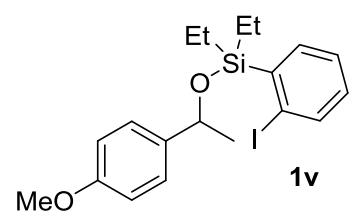

Was prepared according to general procedure $\mathbf{A}$ in $35 \%$ yield. Colorless oil. $\mathbf{R}_{\mathrm{f}}(20: 1$ Hexanes: EtOAc) 0.23. ${ }^{1} \mathrm{H}$ NMR $\left(500 \mathrm{MHz}, \mathrm{CDCl}_{3}\right): \delta \mathrm{ppm} 7.85-7.83(\mathrm{~d}, J=7.70 \mathrm{~Hz}, 1 \mathrm{H}), 7.61-7.60$ $(\mathrm{dd}, J=7.34 \mathrm{~Hz}, J=1.83 \mathrm{~Hz}, 1 \mathrm{H}), 7.34-7.31(\mathrm{td}, J=7.34 \mathrm{~Hz}, J=0.73 \mathrm{~Hz}, 1 \mathrm{H}), 7.30-7.28(\mathrm{~d}, J$ $=8.80 \mathrm{~Hz}, 2 \mathrm{H}), 7.05-7.02(\mathrm{td}, J=7.70 \mathrm{~Hz}, J=1.83 \mathrm{~Hz}, 1 \mathrm{H}), 6.86-6.84(\mathrm{~d}, J=8.80 \mathrm{~Hz}, 2 \mathrm{H})$, 4.96-4.92 (q, 1H), 3.80 (s, 3H), 1.51-1.50 (d, $6.60 \mathrm{~Hz}, 3 \mathrm{H}), 1.06-0.79$ (m, 10H). ${ }^{13} \mathrm{C}$ NMR (126 $\left.\mathrm{MHz}, \mathrm{CDCl}_{3}\right) \delta \mathrm{ppm} 5.2,5.4,6.8,6.9,27.1,55.2,71.2,102.6,113.5,126.2,126.8,130.9$, 
136.4, 138.1, 139.7, 146.9, 158.6. HRMS (EI+) calcd. for $\mathrm{C}_{19} \mathrm{H}_{25} \mathrm{IO}_{2} \mathrm{Si}[\mathrm{M}]$ : 440.0669:, found: 440.0667.

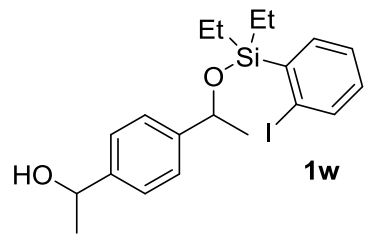

Was prepared according to general procedure A (using 1.6 equiv of $\mathbf{1 A}$ ) in $35 \%$ yield with $7 \%$ of 1x. Colorless oil. $\mathrm{R}_{\mathrm{f}}\left(10: 1\right.$ Hexanes: EtOAc) $0.08 .{ }^{1} \mathrm{H}$ NMR $\left(500 \mathrm{MHz}, \mathrm{CDCl}_{3}\right): \delta$ ppm 7.87 (d, $J=7.9 \mathrm{~Hz}, 1 \mathrm{H}), 7.65$ (d, $J=8.9 \mathrm{~Hz}, 1 \mathrm{H}), 7.39$ (t, $J=7.7 \mathrm{~Hz}, 2 \mathrm{H}), 7.34-7.28$ (m, 3H), 7.10$7.01(\mathrm{~m}, 1 \mathrm{H}), 5.04-5.00(\mathrm{~m}, 1 \mathrm{H}), 4.94-4.90(\mathrm{~m}, 1 \mathrm{H}), 1.56-1.51(\mathrm{~m}, 6 \mathrm{H}), 1.07-0.85(\mathrm{~m}, 10 \mathrm{H})$. ${ }^{13} \mathrm{C}$ NMR (126 MHz, CDCl3) $\delta 5.3,5.5,6.9,7.0,25.1,27.1,70.3,71.4,102.6,125.4,125.7$, $126.9,131.0,138.1,139.7,142.3,144.5,145.7$. HRMS (ESI) calcd. for $\mathrm{C}_{20} \mathrm{H}_{27} \mathrm{IO}_{2} \mathrm{Si}[\mathrm{M}]+\mathrm{Na}$ : 477.0723, found: 477.0721 .

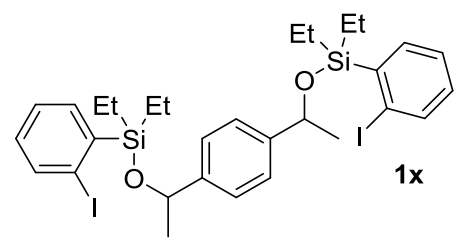

Was prepared according to general procedure A (using 1.6 equiv of $\mathbf{1 A}$ ) in $7 \%$ yield with $35 \%$ of 1w. Colorless oil. $\mathrm{R}_{\mathrm{f}}\left(20: 1\right.$ Hexanes: EtOAc) $0.31 .{ }^{1} \mathrm{H}$ NMR $\left(500 \mathrm{MHz}, \mathrm{CDCl}_{3}\right) \delta 7.87(\mathrm{~d}, J$ $=7.9 \mathrm{~Hz}, 2 \mathrm{H}), 7.65(\mathrm{~d}, J=7.4 \mathrm{~Hz}, 2 \mathrm{H}), 7.35(\mathrm{dd}, J=9.8,4.8 \mathrm{~Hz}, 6 \mathrm{H}), 7.07(\mathrm{t}, J=8.4 \mathrm{~Hz}, 2 \mathrm{H})$, $5.00(\mathrm{q}, J=6.3 \mathrm{~Hz}, 2 \mathrm{H}), 1.56(\mathrm{~d}, J=6.3 \mathrm{~Hz}, 6 \mathrm{H}), 1.08-0.80$ (m, 20H). ${ }^{13} \mathrm{C}$ NMR $(126 \mathrm{MHz}$, $\left.\mathrm{CDCl}_{3}\right) \delta 5.2,5.5,6.8,7.0,27.1,71.5,102.6,125.4,126.8,131.0,138.1,139.7,142.3,144.5$, 145.7. HRMS (EI) calcd. for $\mathrm{C}_{30} \mathrm{H}_{40} \mathrm{I}_{2} \mathrm{O}_{2} \mathrm{Si}_{2}: 742.0657$, found: 742.0657 .

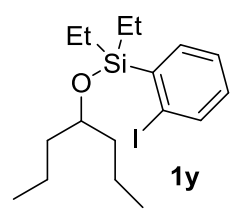

Was prepared according to general procedure $\mathbf{A}$ in 37\% yield. Colorless oil. $\mathrm{R}_{\mathrm{f}}$ (hexanes): 0.39 . ${ }^{1} \mathrm{H}$ NMR $\left(500 \mathrm{MHz}, \mathrm{CDCl}_{3}\right): \delta \mathrm{ppm} 7.86(\mathrm{~d}, J=8.0 \mathrm{~Hz}, 1 \mathrm{H}), 7.68(\mathrm{~d}, J=7.5 \mathrm{~Hz}, 1 \mathrm{H}), 7.36(\mathrm{t}, J$ $=7.5 \mathrm{~Hz}, 1 \mathrm{H}), 7.05(\mathrm{t}, J=8.0 \mathrm{~Hz}, 1 \mathrm{H}), 3.89(\mathrm{p}, J=5.5 \mathrm{~Hz}, 1 \mathrm{H}), 1.57-1.51(\mathrm{~m}, 4 \mathrm{H}), 1.43-1.36$ 
(m, 4H), 1.00-0.90 (m, 16H). ${ }^{13} \mathrm{C}$ NMR (126 MHz, $\left.\mathrm{CDCl}_{3}\right) \delta$ ppm 5.6, 7.1, 14.4, 18.5, 39.2, $72.8,102.7,126.8,130.8,138.2$, 139.6, 143.0. HRMS (CI+) calcd.for $\mathrm{C}_{17} \mathrm{H}_{29} \mathrm{IOSi}[\mathrm{M}]-1$ : 403.0955, found: 403.0947 .

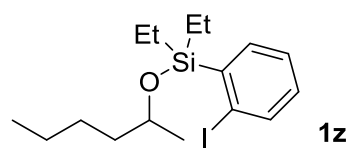

Was prepared according to general procedure $\mathbf{A}$ in $39 \%$ yield. Colorless oil. $\mathbf{R}_{\mathrm{f}}$ (hexanes): 0.38. ${ }^{1} \mathrm{H}$ NMR $\left(500 \mathrm{MHz}, \mathrm{CDCl}_{3}\right): \delta \mathrm{ppm} 7.85(\mathrm{~d}, J=8.0 \mathrm{~Hz}, 1 \mathrm{H}), 7.64(\mathrm{~d}, J=7.5 \mathrm{~Hz}, 1 \mathrm{H}), 7.35(\mathrm{t}, J$ $=7.5 \mathrm{~Hz}, 1 \mathrm{H}), 7.03(\mathrm{t}, J=8.0 \mathrm{~Hz}, 1 \mathrm{H}), 4.01-3.93(\mathrm{~m}, 1 \mathrm{H}), 1.64-1.57(\mathrm{~m}, 1 \mathrm{H}), 1.52-1.45(\mathrm{~m}$, 1H), $1.36-1.28(\mathrm{~m}, 4 \mathrm{H}), 1.24-1.21(\mathrm{~m}, 3 \mathrm{H}), 1.15-1.08$ (m, 2H), 0.99-0.95 (m, 8H), 0.92-0.88 $(\mathrm{m}, 3 \mathrm{H}) .{ }^{13} \mathrm{C} \mathrm{NMR}\left(126 \mathrm{MHz}, \mathrm{CDCl}_{3}\right) \delta \mathrm{ppm} 5.5,7.0,14.1,22.8,23.5,27.8,39.4,69.3,102.7$, 126.8, 130.9, 138.1, 139.7, 142.8. HRMS (EI+) calcd. for $\mathrm{C}_{16} \mathrm{H}_{27} \mathrm{IOSi}[\mathrm{M}]: 390.0876$, found: 390.0878 .

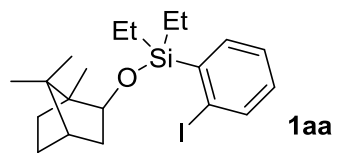

Was prepared according to general procedure $\mathbf{A}$ in $75 \%$ yield. Colorless oil. $\mathbf{R}_{\mathrm{f}}$ (hexanes): 0.33 . ${ }^{1} \mathrm{H}$ NMR $\left(500 \mathrm{MHz}, \mathrm{CDCl}_{3}\right): \delta \mathrm{ppm} 7.84(\mathrm{~d}, J=8.0 \mathrm{~Hz}, 1 \mathrm{H}), 7.69(\mathrm{~d}, J=7.5 \mathrm{~Hz}, 1 \mathrm{H}), 7.36(\mathrm{t}, J$ $=7.5 \mathrm{~Hz}, 1 \mathrm{H}), 7.04(\mathrm{t}, J=8.0 \mathrm{~Hz}, 1 \mathrm{H}), 4.15-4.12(\mathrm{~m}, 1 \mathrm{H}), 2.26-2.17(\mathrm{~m}, 2 \mathrm{H}), 1,79-1.71(\mathrm{~m}$, $1 \mathrm{H}), 1.65-1.63(\mathrm{~m}, 1 \mathrm{H}), 1.34-1.30(\mathrm{~m}, 1 \mathrm{H}), 1.25-1.21(\mathrm{~m}, 1 \mathrm{H}), 1.14-1.07(\mathrm{~m}, 3 \mathrm{H}), 0.98-0.93$ $(\mathrm{m}, 8 \mathrm{H}), 0.88-0.85(\mathrm{~m}, 9 \mathrm{H}) .{ }^{13} \mathrm{C} \mathrm{NMR}\left(126 \mathrm{MHz}, \mathrm{CDCl}_{3}\right) \delta \mathrm{ppm}$ 5.3, 5.7, 7.0, 13.8, 18.9, 20.3, 26.5, 28.4, 39.3, 45.3, 47.4, 50.1, 78.0. 102.6, 126.8, 130.9, 138.2, 139.5, 142.9. HRMS (EI+) calcd. for $\mathrm{C}_{20} \mathrm{H}_{31} \mathrm{IOSi}[\mathrm{M}]:$ 442.1189, found: 442.1181 .

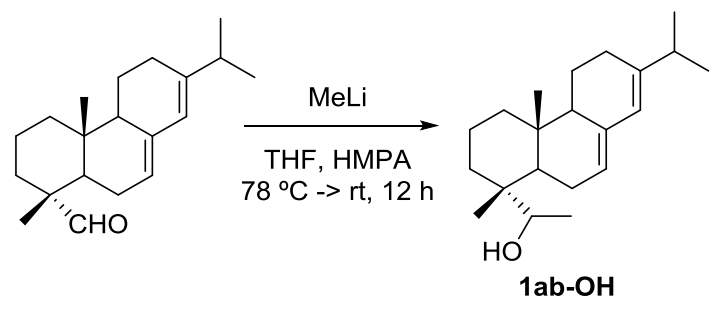

A $25 \mathrm{~mL}$ Schlenk flask under argon was charged with (1R,4aR)-7-isopropyl-1,4a-dimethyl1,2,3,4,4a,4b,5,6,10,10a-decahydrophenanthrene-1-carbaldehyde ${ }^{1}$ (0.85 g, 1 equiv, 2.97 mmol) and THF $(10 \mathrm{~mL})$. The solution was cool to $-78{ }^{\circ} \mathrm{C}$. To this mixture, HMPA $(0.74 \mathrm{~mL}, 4.45$ 
mmol, 1.5 equiv), followed by slow addition of MeLi (2.97 mL, 1.5 equiv, 1.5 M, $4.45 \mathrm{mmol})$ was added dropwise. The reaction was allowed to stir overnight from $-78{ }^{\circ} \mathrm{C}$ to room temperature. A saturated ammonium chloride solution was added and the aqueous phase was extracted with DCM (3 x $50 \mathrm{~mL})$. The organic phase was dried over $\mathrm{Na}_{2} \mathrm{SO}_{4}$, filtered and concentrated. The compound was used for the next step without further purification.

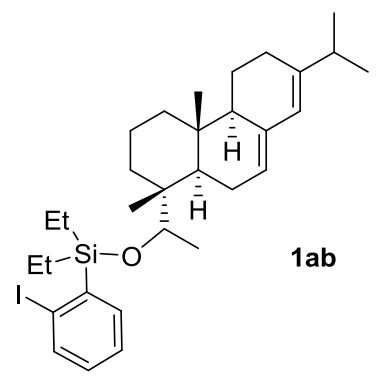

Was prepared according to general procedure B using alcohol 1ab-OH in $26 \%$ yield. $78 \%$ Pure based on NMR. Colorless oil. $\mathrm{R}_{\mathrm{f}}$ (Hexanes) 0.12. ${ }^{1} \mathrm{H}$ NMR $\left(500 \mathrm{MHz}, \mathrm{CDCl}_{3}\right): \delta \mathrm{ppm} 7.85-$ $7.83(\mathrm{~d}, J=8.07 \mathrm{~Hz}, 1 \mathrm{H}), 7.64-7.63(\mathrm{dd}, J=7.34 \mathrm{~Hz}, J=1.47 \mathrm{~Hz}, 1 \mathrm{H}), 7.36-7.32(\mathrm{t}, 1 \mathrm{H}), 7.04-$ $7.01(\mathrm{td}, J=7.70 \mathrm{~Hz}, J=1.83 \mathrm{~Hz}, 1 \mathrm{H}), 5.79(\mathrm{~s}, 1 \mathrm{H}), 5.38(\mathrm{br} \mathrm{s}, 1 \mathrm{H}), 3.91-3.90$ (q, 1H), 2.25$2.22(\mathrm{~m}, 1 \mathrm{H}), 2.09-1.81(\mathrm{~m}, 5 \mathrm{H}), 1.68-1.44(\mathrm{~m}, 4 \mathrm{H}), 1.34-1.21(\mathrm{~m}, 4 \mathrm{H}), 1.15-1.11(\mathrm{~d}, 1 \mathrm{H})$, $1.12-1.08(\mathrm{~m}, 5 \mathrm{H}), 1.04-0.95(\mathrm{~m}, 15 \mathrm{H}), 0.91-0.88(\mathrm{t}, 2 \mathrm{H}), 0.86(\mathrm{~s}, 3 \mathrm{H}), 0.86(\mathrm{~s}, 6 \mathrm{H}), 0.72-0.61$ $(\mathrm{m}, 1 \mathrm{H}) .{ }^{13} \mathrm{C} \mathrm{NMR}\left(126 \mathrm{MHz}, \mathrm{CDCl}_{3}\right) \delta \mathrm{ppm} 5.5,5.6,7.1,14.1,14.2,17.4,18.2,18.6,20.8$, 21.4, 22.7, 23.9, 27.5, 30.9, 31.6, 34.7, 34.8, 34.9, 39.1, 40.8, 44.9, 51.2, 75.8, 102.7, 122.4, 126.8, 130.9, 135.4, 138.1, 139.7, 143.2, 145.3, 148.5. HRMS (ESI) calcd. for $\mathrm{C}_{31} \mathrm{H}_{47} \mathrm{IOSi}$ [M]+1: 590.2457, found: 590.2428.

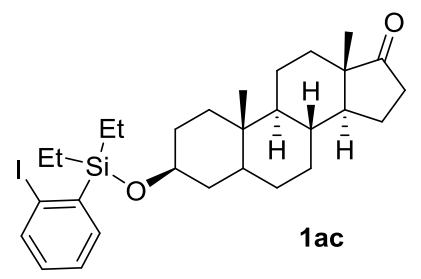

Was prepared according to general procedure $\mathbf{B}$ in $12 \%$ yield. Colorless oil. $\mathrm{R}_{\mathrm{f}}(9: 1$-Hexanes: EtOAc): 0.12. ${ }^{1} \mathrm{H}$ NMR (500 MHz, $\left.\mathrm{CDCl}_{3}\right): \delta \mathrm{ppm} 7.85-7.83(\mathrm{dd}, J=8.07 \mathrm{~Hz}, J=0.73 \mathrm{~Hz}$, $1 \mathrm{H}), 7.60-7.58(\mathrm{dd}, J=7.34 \mathrm{~Hz}, J=1.83 \mathrm{~Hz}, 1 \mathrm{H}), 7.37-7.32(\mathrm{td}, J=7.70 \mathrm{~Hz}, J=1.10 \mathrm{~Hz}, 1 \mathrm{H})$, 7.04-7.01 (td, $J=7.70 \mathrm{~Hz}, J=1.83 \mathrm{~Hz}, 1 \mathrm{H}), 3.72-3.68(\mathrm{~m}, 1 \mathrm{H}), 2.46-2.40(\mathrm{dd}, J=19.44 \mathrm{~Hz}, J$ $=8.80 \mathrm{~Hz}, 1 \mathrm{H}), 2.09-2.02(\mathrm{~m}, 1 \mathrm{H}), 1.94-1.92(\mathrm{~m}, 1 \mathrm{H}), 1.83-1.77(\mathrm{~m}, 2 \mathrm{H}), 1.71-1.63(\mathrm{~m}, 2 \mathrm{H})$, $1.60-1.44(\mathrm{~m}, 5 \mathrm{H}), 1.32-1.17(\mathrm{~m}, 6 \mathrm{H}), 1.12-1.05(\mathrm{~m}, 2 \mathrm{H}), 1.01-0.92(\mathrm{~m}, 10 \mathrm{H}), 0.86(\mathrm{~s}, 6 \mathrm{H})$ 
0.83-0.82 (m, 1H), 0.71-0.57 (m, 2H). ${ }^{13} \mathrm{C}$ NMR (126 MHz, $\left.\mathrm{CDCl}_{3}\right) \delta \mathrm{ppm} 5.5,7.0,12.4,13.8$, 20.5, 21.8, 28.5, 30.9, 31.6, 31.8, 35.1, 35.7, 35.9, 37.1, 38.4, 38.5, 44.9, 45.0, 47.8, 51.5, 54.5, 72.5, 102.7, 126.8, 130.9, 138.0, 139.7, 142.6, 221.4. HRMS (ESI) calcd. for $\mathrm{C}_{29} \mathrm{H}_{44} \mathrm{IO}_{2} \mathrm{Si}$ $[\mathrm{M}]+1:$ 579.2155, found: 579.2145. 


\section{Optimization of the Reaction Parameters}

\section{Table S1: Optimization of Catalyst and Ligands}

\begin{tabular}{|c|c|c|c|c|}
\hline $\begin{array}{l}-1 \\
\mathrm{Si}^{\prime} \\
\mathrm{Et}_{2} \\
1 \mathrm{a}\end{array}$ & $\begin{array}{r}\begin{array}{r}\text { Cataly } \\
\text { Ligar }\end{array} \\
\mathrm{Cs}_{2} \mathrm{C} \\
\mathrm{P}\end{array}$ & $\begin{array}{l}(10 \mathrm{~mol} \%) \\
(20 \mathrm{~mol} \%)\end{array}$ & $\begin{array}{l}-\mathrm{H} \\
\mathrm{Si}^{-} \\
\mathrm{Et}_{2} \\
2 \mathrm{a}\end{array}$ & P \\
\hline entry & Catalyst & Ligand & Conditions & NMR Yield, $\%^{a}$ \\
\hline 1 & $\mathrm{Pd}\left(\mathrm{PPh}_{3}\right)_{4}$ & - & $120^{\circ} \mathrm{C}$ & $0^{c}$ \\
\hline 2 & PEPPSI $^{\mathrm{TM}}-\mathrm{IPr}$ & - & $120^{\circ} \mathrm{C}$ & $0^{b, c}$ \\
\hline 3 & PEPPSI $^{\mathrm{TM}}-\mathrm{IPr}$ & - & $120^{\circ} \mathrm{C}$ & $0^{b, c, d}$ \\
\hline 4 & $\mathrm{Pd}(\mathrm{OAc})_{2}$ & dppf & $120^{\circ} \mathrm{C}$ & $0^{c}$ \\
\hline 5 & $\mathrm{Pd}(\mathrm{OAc})_{2}$ & $\mathbf{L}$ & $120^{\circ} \mathrm{C}$ & $0^{c}$ \\
\hline 6 & $\mathrm{Pd}\left(\mathrm{PPh}_{3}\right)_{4}$ & - & rt, Blue LED & 72 \\
\hline 7 & PEPPSI $^{\mathrm{TM}}$-IPr & - & rt, Blue LED & NR \\
\hline 8 & PEPPSI $^{\mathrm{TM}}$-IPr & - & rt, Blue LED & $N R^{d}$ \\
\hline 9 & $\mathrm{Pd}(\mathrm{OAc})_{2}$ & dppf & rt, Blue LED & 67 \\
\hline 10 & $\mathrm{Pd}(\mathrm{OAc})_{2}$ & $\mathbf{L}$ & rt, Blue LED & 79 \\
\hline 11 & $\mathrm{Pd}(\mathrm{OAc})_{2}$ & xanthphos & rt, Blue LED & 68 \\
\hline 12 & $\mathrm{Pd}(\mathrm{OAc})_{2}$ & bipy & rt, Blue LED & NR \\
\hline 13 & none & $\mathbf{L}$ & rt, Blue LED & NR \\
\hline 14 & $\mathrm{Pd}(\mathrm{OAc})_{2}$ & $\mathbf{L}$ & rt, 23W CFL & 49 \\
\hline
\end{tabular}

${ }^{a} \mathrm{NMR}$ yields were calibrated using $\mathrm{CH}_{2} \mathrm{Br}_{2}$ as an internal standard.

${ }^{\mathrm{b}} 5 \mathrm{~mol} \%$ of catalyst was used. ${ }^{\mathrm{C}} \mathrm{Hydro}$-dehalogenation of $\mathbf{1 a}$.

d 1.1 equiv of $i$-Pr-I was used as an additive. 


\section{Table S2: Optimization of the Silicon Tether}

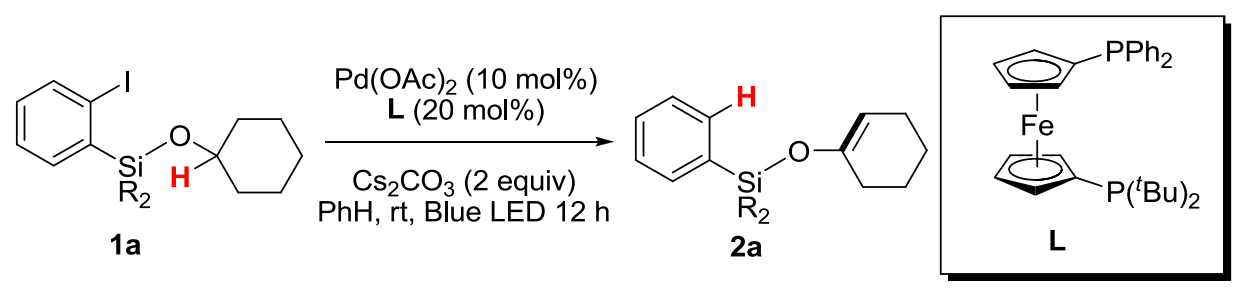

\begin{tabular}{|c|c|c|}
\hline Entry & $\mathrm{R}$ & NMR Yield, $\%^{\mathrm{a}}$ \\
\hline 1 & $\mathrm{Et}$ & $79(79)$ \\
\hline 2 & $\mathrm{Me}$ & $68(25)$ \\
\hline 3 & $i-\mathrm{Pr}$ & $31^{\mathrm{b}}$ \\
\hline
\end{tabular}

${ }^{a} \mathrm{NMR}$ Yield were calibrated using $\mathrm{CH}_{2} \mathrm{Br}_{2}$ as an internal standard. Yields in parentheses are isolated yields. ${ }^{\mathrm{b}} 19 \%$ of hydro-dehalogenation was observed.

A brief optimization of the silyl tether indicated that both dimethyl- (entry 2) and diisopropyl (entry 3) substituted substrates were less efficient compared with diethyl (entry 1). When dimethyl tether derivative was used, the desaturation product decomposed rapidly upon isolation (entry 2). Surprisingly, the diisopropyl-tethered derivative provided very low yield of the desaturation product, presumably due to a low degree of flexibility during the HAT transition state that renders the oxidation process uneventful (entry 3$)^{2,3}$ 


\section{Oxidation of Silyl Ethers}

\section{General procedure for oxidation of silyl ethers:}
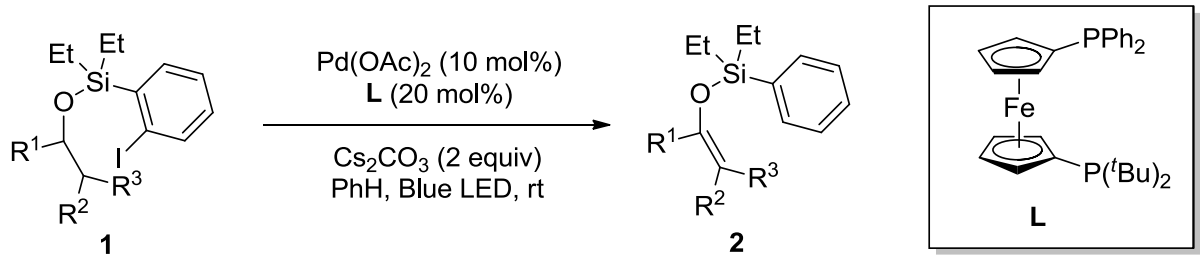

An oven dried $3 \mathrm{~mL}$ Wheaton $\mathrm{V}$-vial containing a stirring bar was charged with silyl ethers 1 (0.2 mmol), $\mathrm{Pd}(\mathrm{OAc})_{2}(4.49 \mathrm{mg}, 0.02 \mathrm{mmol})$, ligand $\mathbf{L}(20.6 \mathrm{mg}, 0.04 \mathrm{mmol})$ and $\mathrm{Cs}_{2} \mathrm{CO}_{3}$ (130 mg, $0.4 \mathrm{mmol}$ ) under $\mathrm{N}_{2}$ atmosphere (glovebox). Dry benzene ( $2 \mathrm{~mL}$ ) was added and the reaction vessel was capped with a pressure screw cap. The vial was irradiated with $34 \mathrm{~W}$ Blue LED lamp (Kessil KSH150B LED Grow Light) for 12-24 h (monitored by GC/MS), with cooling from a fan (vial temperature reached $37^{\circ} \mathrm{C}$ ). The vial distance from the lamp was about $2-3 \mathrm{~cm}$. The resulting mixture was diluted with hexane $(10 \mathrm{~mL})$, filtered (Celite), and concentrated under a reduced pressure. The residue was purified by filtration through deactivated silica gel pad/column (hexanes: triethylamine $=50: 1$ ) affording the corresponding silyl enol ethers products (2).

\section{Grams scale synthesis: Oxidation of silyl ethers 10}

An oven dried $25 \mathrm{~mL}$ Erlenmeyer flask containing a stirring bar was charged with silyl ethers $10(3 \mathrm{mmol}), \mathrm{Pd}(\mathrm{OAc})_{2}(64.4 \mathrm{mg}, 0.3 \mathrm{mmol})$, ligand $\mathbf{L}(309 \mathrm{mg}, 0.6 \mathrm{mmol})$ and $\mathrm{Cs}_{2} \mathrm{CO}_{3}(1955 \mathrm{mg}, 6 \mathrm{mmol})$ under $\mathrm{N}_{2}$ atmosphere (glovebox). Dry benzene $(20 \mathrm{~mL})$ was added and the reaction flask was capped with a septum under argon ballon. The flask was irradiated with $34 \mathrm{~W}$ Blue LED lamp (Kessil KSH150B LED Grow Light) for $24 \mathrm{~h}$ (monitored by GC/MS), with cooling from a fan (vial temperature reached $37{ }^{\circ} \mathrm{C}$ ). The flask distance from the lamp was about $2-3 \mathrm{~cm}$. The resulting mixture was diluted with hexane (10 $\mathrm{mL}$ ), filtered (Celite), and concentrated under a reduced pressure. The residue was purified by filtration deactivated short column (hexanes: triethylamine $=50: 1$ ) to affording the corresponding silyl enol ethers product $20781 \mathrm{mg}, 99 \%$ yield. 


\section{Diagram of Reaction Set-Up:}

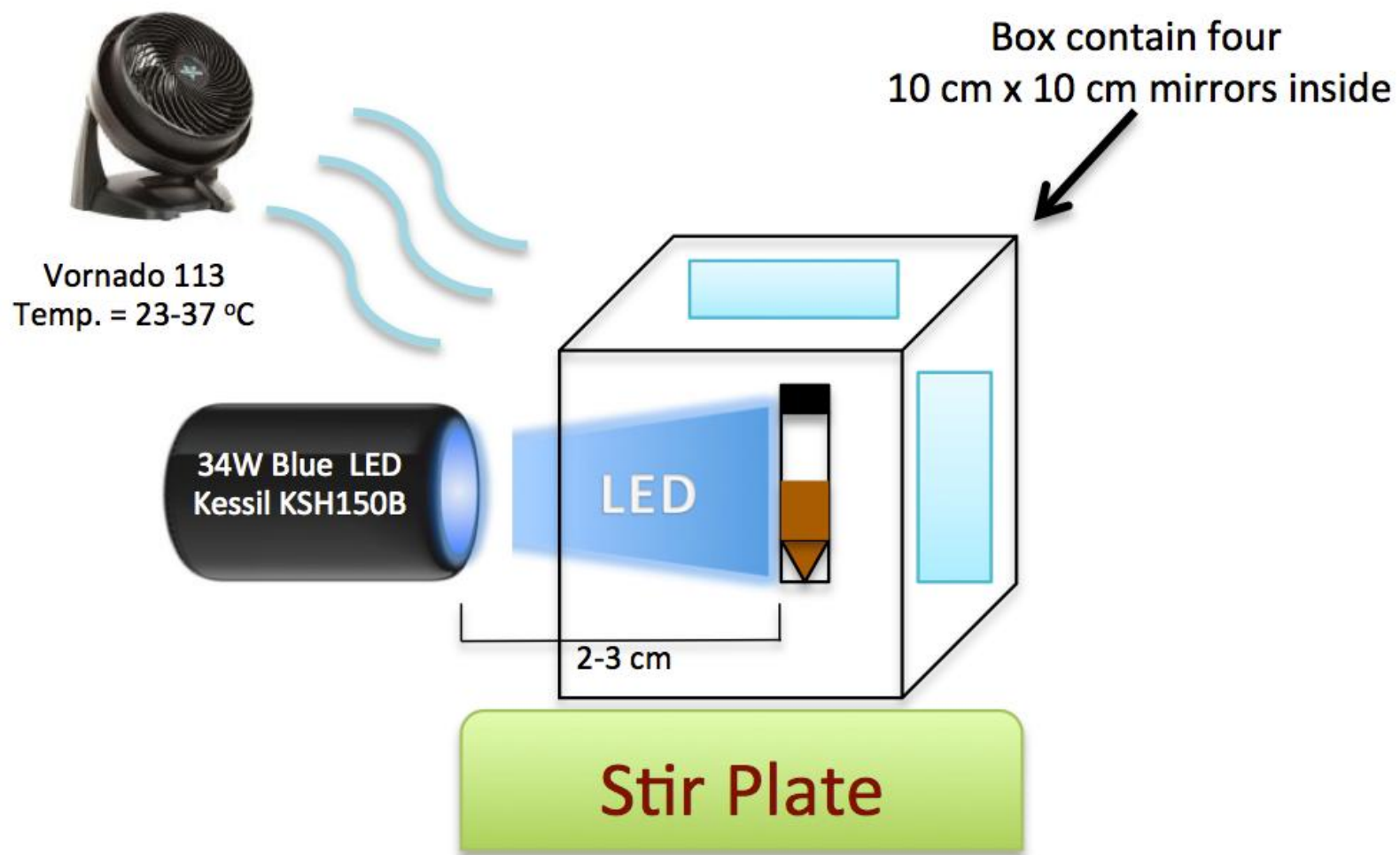

Silyl Enol Ether (2) Analytics:

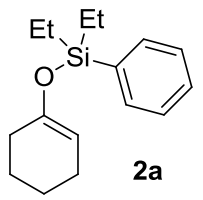

Was prepared according to the general procedure in $79 \%$ yield. Yellow oil. $\mathrm{R}_{\mathrm{f}}$ (hexanes): 0.34. ${ }^{1} \mathrm{H}$ NMR $\left(500 \mathrm{MHz}, \mathrm{CDCl}_{3}\right): \delta$ ppm 7.60-7.58 (m, 2H), 7.39-7.36 (m, 3H), 4.88-4.86 (m, $1 \mathrm{H}), 2.04-2.01(\mathrm{~m}, 2 \mathrm{H}), 1.98-1.95(\mathrm{~m}, 2 \mathrm{H}), 1.66-1.61(\mathrm{~m}, 2 \mathrm{H}), 1.51-1.46(\mathrm{~m}, 2 \mathrm{H}), 1.14-1.07$ $(\mathrm{m}, 2 \mathrm{H}), 1.03-0.90(\mathrm{~m}, 8 \mathrm{H}) .{ }^{13} \mathrm{C}$ NMR $\left(126 \mathrm{MHz}, \mathrm{CDCl}_{3}\right) \delta \mathrm{ppm} 6.6,6.7,22.3,23.2,23.8$, 29.8, 104.3, 127.7, 129.4, 133.9, 136.4, 150.4. HRMS (ESI) calcd. for $\mathrm{C}_{16} \mathrm{H}_{24} \mathrm{OSi}[\mathrm{M}]+1$ : 261.1677 , found: 261.1675 . 


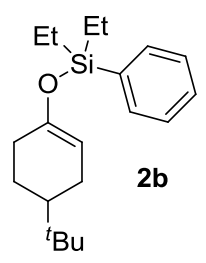

Was prepared according to the general procedure in $65 \%$ yield. Yelllow oil. $R_{\mathrm{f}}$ (hexanes): 0.35 . ${ }^{1} \mathrm{H}$ NMR (500 MHz, $\mathrm{CDCl}_{3}$ ): $\delta$ ppm 7.60-7.58 (m, 2H), 7.40-7.7.36 (m, 3H), 4.85-4.85 (m, 1H), 2.14-2.03 (m, 2H), 1.80-1.77 (m, 3H), 1.27-1.22 (m, 2H), 1.12-0.97 (m, 10H), 0.86-0.83 (s, 9H). ${ }^{13} \mathrm{C}$ NMR $\left(126 \mathrm{MHz}, \mathrm{CDCl}_{3}\right) \delta$ ppm 5.5, 7.0, 24.4, 25.1, 27.3, 27.6, 30.8, 43.9, 103.9, 127.7, 129.4, 133.6, 133.9, 150.3. HRMS (ESI) calcd. for $\mathrm{C}_{20} \mathrm{H}_{32} \mathrm{OSi}[\mathrm{M}]+1$ : 317.2301, found: 317.2311 .

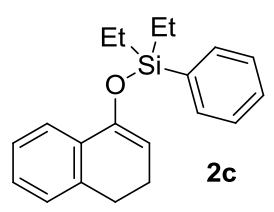

Was prepared according to the general procedure in $86 \%$ yield. Yellow oil. $\mathrm{R}_{\mathrm{f}}($ hexanes/EtOAc = 20/1): 0.31. ${ }^{1} \mathrm{H}$ NMR (500 MHz, $\left.\mathrm{CDCl}_{3}\right): \delta \mathrm{ppm} 7.68-7.65(\mathrm{~m}, 2 \mathrm{H}), 7.58(\mathrm{~d}, J=7.3 \mathrm{~Hz}, 1 \mathrm{H})$, 7.44-7.39 (m, 3H), 7.24-7.22 (m, 1H), 7.19-7.16 (m, 1H), $7.12(\mathrm{~d}, J=7.3,1 \mathrm{H}), 5.12(\mathrm{t}, J=4.4$ $\mathrm{Hz}, 1 \mathrm{H}), 2.75(\mathrm{t}, J=8.1 \mathrm{~Hz}, 2 \mathrm{H}), 2.29-2.24(\mathrm{~m}, 2 \mathrm{H}), 1.07-1.03(\mathrm{~m}, 10 \mathrm{H}) .{ }^{13} \mathrm{C} \mathrm{NMR}(126 \mathrm{MHz}$, $\left.\mathrm{CDCl}_{3}\right): \delta$ ppm 5.6, 6.7, 22.1, 28.1, 104.7, 121.8, 126.2, 126.9, 127.3, 127.8, 129.6, 133.4, 133.9, 135.9, 137.1, 148.1. HRMS (ESI) calcd. for $\mathrm{C}_{20} \mathrm{H}_{24} \mathrm{OSi}[\mathrm{M}]+1$ : 309.1675, found: 309.1685 .

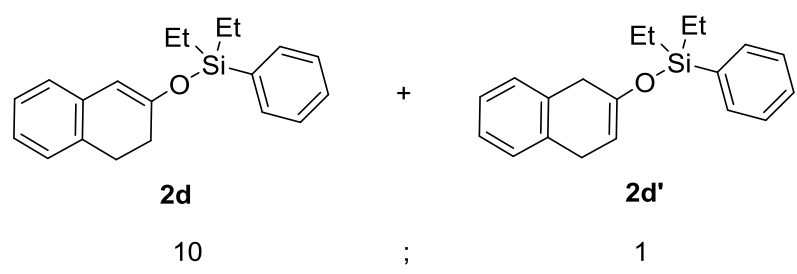

Was prepared according to the general procedure in $72 \%$ yield. Yellow oil. $\mathrm{R}_{\mathrm{f}}$ (hexanes/EtOAc = 20/1): 0.31. ${ }^{1} \mathrm{H}$ NMR (500 MHz, $\left.\mathrm{CDCl}_{3}\right): \delta$ ppm 7.64-7.62 (m, 2H), 7.43-7.40 (m, 2H), 7.09$7.7 .05(\mathrm{~m}, 3 \mathrm{H}), 7.00-6.97(\mathrm{~m}, 1 \mathrm{H}), 6.83(\mathrm{~m}, J=7.3,1 \mathrm{H}), 5.68(\mathrm{~s}, 1 \mathrm{H}), 2.88(\mathrm{t}, J=8.0 \mathrm{~Hz}, 2 \mathrm{H})$, $2.41(\mathrm{t}, J=8.0 \mathrm{~Hz}, 2 \mathrm{H}), 1.09-1.00(\mathrm{~m}, 10 \mathrm{H}) .{ }^{13} \mathrm{C} \mathrm{NMR}\left(126 \mathrm{MHz}, \mathrm{CDCl}_{3}\right): \delta \mathrm{ppm} 5.5,6.6$, 28.9, 29.1, 106.0, 124.7, 126.4, 126.9, 127.8, 127.9, 129.8, 131.9, 133.8, 134.0, 135.6, 155.3. HRMS (EI) calcd. for $\mathrm{C}_{20} \mathrm{H}_{24} \mathrm{OSi}[\mathrm{M}]: 308.1597$, found: 308.1606 . 


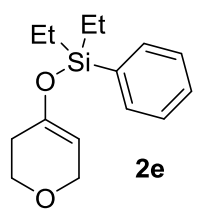

Was prepared according to the general procedure in $74 \%$ yield. Colorless oil. $\mathrm{R}_{\mathrm{f}}$ (hexanes): 0.06. ${ }^{1} \mathrm{H}$ NMR (400 MHz, $\mathrm{CDCl}_{3}$ ): $\delta$ ppm 7.59-7.57 (m, 2H), 7.40-7.38 (m, 3H), 4.81-4.79 (m, $1 \mathrm{H}), 4.10-4.08(\mathrm{~m}, 2 \mathrm{H}), 3.79(\mathrm{t}, J=5.5,2 \mathrm{H}), 2.17-2.15(\mathrm{~m}, 2 \mathrm{H}), 1.05-0.95(\mathrm{~m}, 10 \mathrm{H}) .{ }^{13} \mathrm{C} \mathrm{NMR}$ $\left(100 \mathrm{MHz} \mathrm{CDCl}_{3}\right) \delta \mathrm{ppm} 5.4,6.6,30.3,64.6,102.3,127.8,129.7,133.8,135.7,147.8$. HRMS (AP+) calcd. for $\mathrm{C}_{15} \mathrm{H}_{22} \mathrm{O}_{2} \mathrm{Si}[\mathrm{M}]+1: 263.1467$, found: 263.1467 .

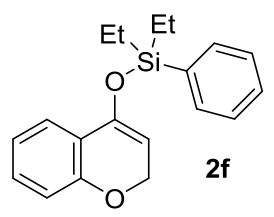

Was prepared according to the general procedure in $75 \%$ yield. Yellow oil. $\mathrm{R}_{\mathrm{f}}$ (hexanes/EtOAc = 20/1): 0.30. ${ }^{1} \mathrm{H}$ NMR (500 MHz, $\left.\mathrm{CDCl}_{3}\right): \delta \mathrm{ppm}$ 7.64-7.63 (m, 2H), 7.47-7.35 (m, 5H), 7.17$7.14(\mathrm{~m}, 1 \mathrm{H}), 6.95-6.91(\mathrm{~m}, 1 \mathrm{H}), 6.80-6.78(\mathrm{~m}, 1 \mathrm{H}), 4.77$ (broad s, 2H), 1.06-1.05 (m, 6H), 0.99-0.95 (m, 2H). $\left.{ }^{13} \mathrm{C} \mathrm{NMR} \mathrm{(126} \mathrm{MHz,} \mathrm{CDCl}_{3}\right) \delta$ ppm 5.4, 6.6, 6.6, 65.7, 97.9, 115.4, 120.9, 122.5, 127.6, 127.8, 128.0, 129.6, 129.8, 133.6, 133.8, 155.6. HRMS (ESI) calcd. for $\mathrm{C}_{19} \mathrm{H}_{22} \mathrm{O}_{2} \mathrm{Si}[\mathrm{M}]+1: 311.1467$, found: 311.1470.

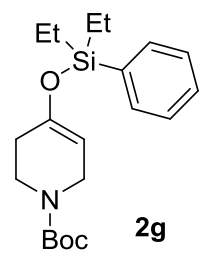

Was prepared according to the general procedure in 58\% yield. Colorless oil. (hexanes:EtOAc = 20:1): 0.23. ${ }^{1} \mathrm{H}$ NMR (500 MHz, $\left.\mathrm{CDCl}_{3}\right): \delta \mathrm{ppm} 7.58$ (m, 1H), 7.41-7.36 (m, 3H), 4.77 (br. s, 1H), 3.82 (br. s, 2H), 3.50 (br. s, 2H), 2.14 (br. s, 2H), 1.45 (s, 9H), 1.03-0.99 (m, 6H), 0.96$0.93(\mathrm{~m}, 4 \mathrm{H}) .{ }^{13} \mathrm{C}$ NMR $\left(100 \mathrm{MHz}, \mathrm{CDCl}_{3}\right) \delta \mathrm{ppm}$ 5.3, 6.6, 28.4, 29.8, 29.9, 41.2, 67.9, 79.5, 127.7, 127.8, 129.7, 133.8, 135.7, 154.9. LRMS expected for $\mathrm{C}_{20} \mathrm{H}_{31} \mathrm{NO}_{3} \mathrm{Si}=361.2$, found: 361.3 HRMS (CI) for $\mathrm{C}_{20} \mathrm{H}_{31} \mathrm{NO}_{3} \mathrm{Si}$ : 435.0642, found: 435.0639. 


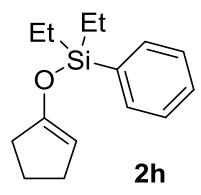

Was prepared according to the general procedure in $65 \%$ yield. Clear and Colorless oil. $\mathrm{R}_{\mathrm{f}}$ (Hexanes): 0.20. ${ }^{1} \mathrm{H}$ NMR (400 MHz, $\left.\mathrm{CDCl}_{3}\right): \delta \mathrm{ppm}$ 7.60-7.58 (m, 2H) 7.41-7.36 (m, 3H), 4.59-4.58 (m, 1H), 2.30-2.21 (m, 4H), 1.85-1.82 (m, 2H), 1.05-0.90 (m, 10H). ${ }^{13} \mathrm{C}$ NMR (100 $\left.\mathrm{MHz}, \mathrm{CDCl}_{3}\right) \delta \mathrm{ppm} 5.3,6.6,21.3,28.7,33.4,102.6,127.8,129.6,133.8,135.7,154.9$. HRMS (AP+) calcd. for $\mathrm{C}_{15} \mathrm{H}_{21} \mathrm{OSi}$ [M]-1: 245.1362, found: 245.1360.

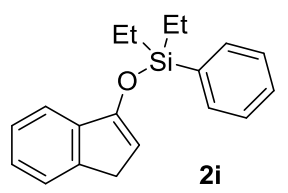

Was prepared according to the general procedure in $70 \%$ yield. Yellow oil. $\mathrm{R}_{\mathrm{f}}$ (hexanes/EtOAc $=20 / 1): 0.31 .{ }^{1} \mathrm{H}$ NMR $\left(500 \mathrm{MHz}, \mathrm{CDCl}_{3}\right): \delta$ ppm 7.69-7.67 (m, 2H), 7.50-7.48 (m, 1H), 7.44$7.39(\mathrm{~m}, 4 \mathrm{H}), 7.35-7.32(\mathrm{~m}, 1 \mathrm{H}), 7.25-7.22(\mathrm{~m}, 1 \mathrm{H}), 5.32(\mathrm{t}, J=2.6 \mathrm{~Hz}, 1 \mathrm{H}), 3.24(\mathrm{~d},=2.6 \mathrm{~Hz}$, $2 \mathrm{H}), 1.08-1.07(\mathrm{~m}, 10 \mathrm{H}) .{ }^{13} \mathrm{C}$ NMR $\left(126 \mathrm{MHz}, \mathrm{CDCl}_{3}\right): \delta \mathrm{ppm} 5.3,6.6,34.0,106.0,118.2$, 123.7, 125.3, 126.0, 127.9, 129.8, 133.9, 135.4, 141.7, 142.7, 153.4. HRMS (EI) calcd. for $\mathrm{C}_{19} \mathrm{H}_{22} \mathrm{OSi}[\mathrm{M}]+1: 295.1518$, found: 295.1519 .

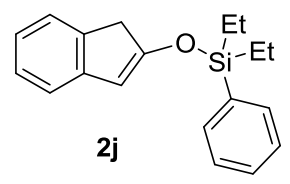

Was prepared according to the general procedure in 58\% yield. Yellow oil. $\mathrm{R}_{\mathrm{f}}$ (hexanes/EtOAc = 20/1): 0.31. ${ }^{1} \mathrm{H}$ NMR (500 MHz, $\left.\mathrm{CDCl}_{3}\right): \delta$ ppm 7.64-7.63 (m, 2H), 7.45-7.39 (m, 3H), 7.25$7.24(\mathrm{~m}, 1 \mathrm{H}), 7.16-7.14(\mathrm{~m}, 1 \mathrm{H}), 7.09-7.07(\mathrm{~m}, 1 \mathrm{H}), 7.03-7.00(\mathrm{~m}, 1 \mathrm{H}), 5.71(\mathrm{~s}, 1 \mathrm{H}), 3.33(\mathrm{~m}$, 2H), 1.01-1.06 (m, 10H). ${ }^{13} \mathrm{C}$ NMR $\left(126 \mathrm{MHz}, \mathrm{CDCl}_{3}\right): \delta \mathrm{ppm} 5.2,6.5,39.4,106.9,119.1$, 122.5, 123.1, 124.6, 127.6, 128.0, 130.0, 133.6, 133.9, 136.7, 145.1. LRMS expected for $\mathrm{C}_{19} \mathrm{H}_{22} \mathrm{OSi}[\mathrm{M}]: 294.5$, found: 294.1 . 


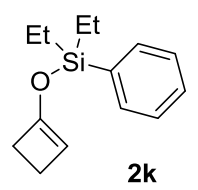

Was prepared according to the general procedure in $43 \%$ yield. Purity $=90 \%$. Light yellow oil. $\mathrm{R}_{\mathrm{f}}$ (Hexanes): 0.20. ${ }^{1} \mathrm{H}$ NMR (500 MHz, $\mathrm{CDCl}_{3}$ ): $\delta \mathrm{ppm}$ 7.60-7.57 (m, 2H) 7.41-7.36 (m, 3H), 4.53-4.52 (d, $J=4.40 \mathrm{~Hz}, 1 \mathrm{H}), 2.56-2.54$ (q, 2H), 1.97-1.95 (q, 2H), 1.04-1.00 (m, 5H), 0.980.93(m, 5H). ${ }^{13} \mathrm{C}$ NMR (126 MHz, $\left.\mathrm{CDCl}_{3}\right) \delta \mathrm{ppm}$ 5.2, 6.5, 19.6, 34.1, 103.4, 127.8, 129.8, 133.8, 135.1, 147.9. HRMS (EI) calcd. for $\mathrm{C}_{14} \mathrm{H}_{21} \mathrm{OSi}[\mathrm{M}]+1: 233.1351$, found: 233.1362 .

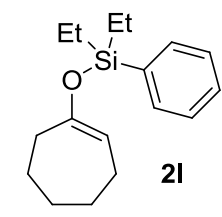

Was prepared according to the general procedure in $61 \%$ yield. Light yellow oil. $\mathrm{R}_{\mathrm{f}}$ (Hexanes): 0.17. ${ }^{1} \mathrm{H}$ NMR (400 MHz, $\left.\mathrm{CDCl}_{3}\right): \delta$ ppm 7.60-7.58 (m, 2H) 7.41-7.35 (m, 3H), 5.02-4.99 (t, $1 \mathrm{H}), 2.28-2.26(\mathrm{~m}, 2 \mathrm{H}), 1.96-1.93(\mathrm{~m}, 2 \mathrm{H}), 1.68-1.63(\mathrm{~m}, 2 \mathrm{H}), 1.56-1.47(\mathrm{~m}, 4 \mathrm{H}), 1.03-0.86$ $(\mathrm{m}, 10 \mathrm{H}) .{ }^{13} \mathrm{C}$ NMR $\left(100 \mathrm{MHz}, \mathrm{CDCl}_{3}\right) \delta \mathrm{ppm} 5.4,6.7,25.2,25.3,31.4,35.4,108.3,127.7$, 129.4, 133.9, 136.3, 156.0. HRMS (AP+) calcd. for $\mathrm{C}_{17} \mathrm{H}_{27} \mathrm{OSi}[\mathrm{M}]+1$ : 275.1831, found: 275.1836

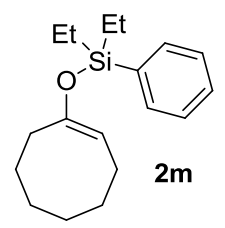

Was prepared according to the general procedure in $72 \%$ yield. Light yellow oil. $R_{\mathrm{f}}(20: 1$ Hexanes: EtOAc) 0.31. ${ }^{1} \mathrm{H} \mathrm{NMR}\left(500 \mathrm{MHz} \mathrm{CDCl}_{3}\right): \delta \mathrm{ppm}$ 7.62-7.58 (m, 2H) 7.39-7.35 (m, $3 \mathrm{H}), 4.68-4.72(\mathrm{~m}, 1 \mathrm{H}), 2.22-2.20(\mathrm{~m}, 2 \mathrm{H}), 1.98-1.96(\mathrm{~m}, 2 \mathrm{H}), 1.60-1.57(\mathrm{~m}, 2 \mathrm{H}), 1.50-1.43$ $(\mathrm{m}, 6 \mathrm{H}), 1.02-0.85(\mathrm{~m}, 10 \mathrm{H}) .{ }^{13} \mathrm{C}$ NMR $\left(126 \mathrm{MHz}, \mathrm{CDCl}_{3}\right) \delta \mathrm{ppm} 5.4,6.7,25.5,26.3,26.4$, $27.9,30.9,31.0,105.1,127.7,129.4,133.9,136.3,153.0$. HRMS (AP+) calcd. for $\mathrm{C}_{18} \mathrm{H}_{29} \mathrm{OSi}$ [M]+1: 289.1988, found: 289.1995 . 


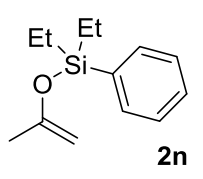

Was prepared according to the general procedure in $79 \%$ yield. Colorless oil. $\mathrm{R}_{\mathrm{f}}$ (50:1Hexanes: EtOAc): 0.20. ${ }^{1} \mathrm{H}$ NMR $\left(500 \mathrm{MHz}, \mathrm{CDCl}_{3}\right): \delta \mathrm{ppm} 7.61-7.59(\mathrm{dd}, J=7.34 \mathrm{~Hz}, J=$ $1.83 \mathrm{~Hz}, 1 \mathrm{H}), 7.40-7.38$ (m, 3H), 4.04 (s, 2H), 1.82 (s, 3H), 1.05-1.01 (m, 6H), 0.99-0.94 (m, $4 \mathrm{H}) .{ }^{13} \mathrm{C}$ NMR $\left(126 \mathrm{MHz}, \mathrm{CDCl}_{3}\right) \delta \mathrm{ppm} \mathrm{5.3,6.6,} \mathrm{22.7,} \mathrm{91.3,} \mathrm{127.8,} \mathrm{129.6,} \mathrm{133.8,} \mathrm{135.8,}$ 155.9. LRMS expected for $\mathrm{C}_{13} \mathrm{H}_{20} \mathrm{OSi}=220.4$, found: 220.1 .

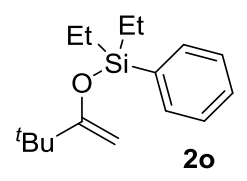

Was prepared according to the general procedure in $95 \%$ yield. Colorless oil. $\mathrm{R}_{\mathrm{f}}$ (hexanes): 0.33. ${ }^{1} \mathrm{H}$ NMR (400 MHz, $\left.\mathrm{CDCl}_{3}\right): \delta \mathrm{ppm}$ 7.63-7.61 (m, 2H), 7.42-7.38 (m, 3H), $4.06(\mathrm{~d}, J=$ $1.6 \mathrm{~Hz}, 1 \mathrm{H}), 3.89$ (d, $J=1.6 \mathrm{~Hz}, 1 \mathrm{H}), 1.16(\mathrm{~s}, 9 \mathrm{H}), 1.06-0.99(\mathrm{~m}, 7 \mathrm{H}), 0.91-0.91(\mathrm{~m}, 3 \mathrm{H}) .{ }^{13} \mathrm{C}$ NMR (100 MHz, $\left.\mathrm{CDCl}_{3}\right) \delta$ ppm 5.5, 6.7, 28.2, 36.8, 85.5, 127.7, 129.4, 133.8, 166.8. HRMS (AP+) calcd. for $\mathrm{C}_{16} \mathrm{H}_{26} \mathrm{OSi}[\mathrm{M}]+1: 263.1831$, found: 263.1839 .

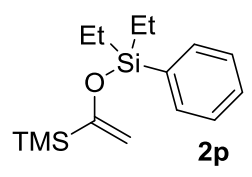

Was prepared according to the general procedure in 97\% yield. Brown oil. $\mathrm{R}_{\mathrm{f}}$ (hexanes): 0.31 . ${ }^{1} \mathrm{H}$ NMR (500 MHz, $\mathrm{CDCl}_{3}$ ): $\delta$ ppm 7.61-7.59 (m, 2H), 7.41-7.38 (m, 3H), $4.78(\mathrm{~s}, 1 \mathrm{H}), 4.51$ (s, 1H), 1.05-0.96 (m, 10H), 0.15 (s, 9H). ${ }^{13} \mathrm{C}$ NMR $\left(126 \mathrm{MHz}, \mathrm{CDCl}_{3}\right) \delta \mathrm{ppm}$ 2.3, 5.8, 6.7, 103.9, 127.7, 129.4, 133.9, 136.5, 166.8 HRMS (AP+) calcd. for $\mathrm{C}_{15} \mathrm{H}_{26} \mathrm{OSi}[\mathrm{M}]+1: 279.1600$, found: 279.1611.

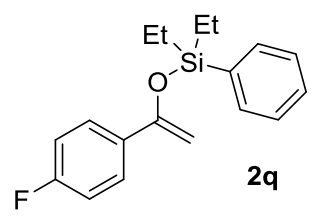

Was prepared according to the general procedure in $72 \%$ yield. Colorless oil. $\mathrm{R}_{\mathrm{f}}$ (20:1Hexanes: EtOAc): 0.20. ${ }^{1} \mathrm{H}$ NMR (500 MHz, $\left.\mathrm{CDCl}_{3}\right): \delta \mathrm{ppm}$ 7.64-7.62 (m, 4H), 7.42-7.40 (m, $3 \mathrm{H}), 7.04-7.00(\mathrm{t}, 2 \mathrm{H}), 4.78-4.78(\mathrm{~d}, J=1.83 \mathrm{~Hz}, 1 \mathrm{H}), 4.33-4.33(\mathrm{~d}, J=1.83 \mathrm{~Hz}, 1 \mathrm{H}), 1.05-$ 
$1.04(\mathrm{~d}, 10 \mathrm{H}) .{ }^{13} \mathrm{C} \mathrm{MR}\left(126 \mathrm{MHz}, \mathrm{CDCl}_{3}\right) \delta \mathrm{ppm} 5.3,6.6,90.5,114.9,115.0,126.9,127.0$, 127.9, 129.8, 133.9, 135.3, 154.7, 161.9, 163.8. HRMS (EI) calcd. for $\mathrm{C}_{18} \mathrm{H}_{21} \mathrm{FOSi}$ : 300.1342, found: 300.1346.

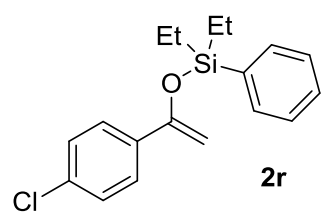

Was prepared according to the general procedure in $85 \%$ yield. Colorless oil. $\mathrm{R}_{\mathrm{f}}(20: 1$ Hexanes: EtOAc): 0.20. ${ }^{1} \mathrm{H}$ NMR $\left(500 \mathrm{MHz}, \mathrm{CDCl}_{3}\right): \delta \mathrm{ppm} 7.62-7.60(\mathrm{dd}, J=7.70 \mathrm{~Hz}, J=$ $1.83 \mathrm{~Hz}, 2 \mathrm{H}), 7.57-7.58(\mathrm{~d}, J=8.44 \mathrm{~Hz}, 2 \mathrm{H}), 7.40-7.39(\mathrm{~d}, J=7.34 \mathrm{~Hz}, 2 \mathrm{H}), 7.31-7.27(\mathrm{~m}$, $3 \mathrm{H}), 4.83-4.83(\mathrm{~d}, J=2.30 \mathrm{~Hz}, 1 . \mathrm{H}), 4.36-4.35(\mathrm{~d}, J=1.83 \mathrm{~Hz}, 1 \mathrm{H}), 1.03(\mathrm{~s}, 10 \mathrm{H}) .{ }^{13} \mathrm{C}$ NMR $\left(126 \mathrm{MHz}, \mathrm{CDCl}_{3}\right) \delta \mathrm{ppm} 5.3,6.6,90.5,114.9,115.0,127.0,127.9,129.8,133.8,135.3,154.7$, 163.8. LRMS expected for $\mathrm{C}_{18} \mathrm{H}_{21} \mathrm{ClOSi}$ : 316.9 , found: 316.1 .

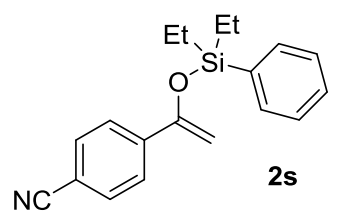

Was prepared according to the general procedure in 50\% yield. Purity $=88 \%$ based on GCMS. Light yellow oil. $\mathrm{R}_{\mathrm{f}}\left(20: 1-\mathrm{Hexanes}\right.$ EtOAc): 0.18. ${ }^{1} \mathrm{H} \mathrm{NMR}\left(500 \mathrm{MHz}, \mathrm{CDCl}_{3}\right): \delta \mathrm{ppm}$ 7.74-7.72 (d, $J=8.44 \mathrm{~Hz}, 2 \mathrm{H}), 7.63-7.59(\mathrm{dd},, J=11.34, J=8.44 \mathrm{~Hz}, 4 \mathrm{H}), 7.40-7.39$ (m, 3H), 4.96-4.96 (d, $J=2.57 \mathrm{~Hz}, 1 . \mathrm{H}), 4.50-4.49(\mathrm{~d}, J=2.57 \mathrm{~Hz}, 1 \mathrm{H}), 1.04(\mathrm{~s}, 10 \mathrm{H}) .{ }^{13} \mathrm{C}$ NMR $(126$ $\left.\mathrm{MHz}, \mathrm{CDCl}_{3}\right) \delta \mathrm{ppm} 5.2,6.6,93.7,111.6,118.9,125.7,128.0,129.9,132.0,133.8,134.8$, 141.7, 153.8. LRMS expected for $\mathrm{C}_{19} \mathrm{H}_{21} \mathrm{NOSi}$ : 307.5, found: 306.9 .

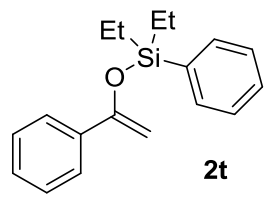

Was prepared according to the general procedure in $64 \%$ yield. Yellow oil. $\mathrm{R}_{\mathrm{f}}$ (hexanes): 0.27 . ${ }^{1} \mathrm{H}$ NMR (500 MHz, $\mathrm{CDCl}_{3}$ ): $\delta$ ppm 7.68-7.63 (m, 4H), 7.43-7.39 (m, 3H), 7.35-7.31 (m, 3H), $4.87(\mathrm{~d}, J=2.0 \mathrm{~Hz}, 1 \mathrm{H}), 4.36(\mathrm{~d}, J=2.0 \mathrm{~Hz}, 1 \mathrm{H}), 1.05(\mathrm{~s}, 10 \mathrm{H}) .{ }^{13} \mathrm{C} \mathrm{NMR}\left(126 \mathrm{MHz}, \mathrm{CDCl}_{3}\right)$ $\delta$ ppm 5.4, 6.4, 6.7, 90.7, 125.1, 125.3, 127.9, 128.1, 128.2, 129.7, 134.0, 135.5, 137.5, 155.6, 184.3. LRMS expected for $\mathrm{C}_{18} \mathrm{H}_{22} \mathrm{OSi}[\mathrm{M}]: 282.1$, found: 282.1 . 


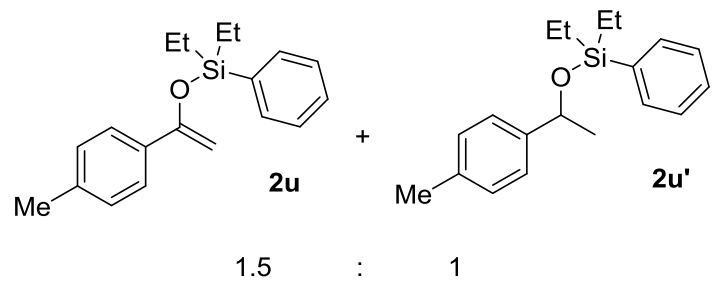

Was prepared according to the general procedure in $77 \%$ yield. Major product $\mathbf{2 u}=45 \%$. Light Yellow oil. $\mathrm{R}_{\mathrm{f}}\left(20: 1\right.$ Hexanes: EtOAc): 0.20. ${ }^{1} \mathrm{H}$ NMR $\left(500 \mathrm{MHz}, \mathrm{CDCl}_{3}\right): \delta$ ppm 7.66-7.64 $(\mathrm{dd},, J=9.13, J=7.70 \mathrm{~Hz}, 2 \mathrm{H}) 7.58-7.56(\mathrm{~d}, J=8.07 \mathrm{~Hz}, 2 \mathrm{H}), 7.43-7.38(\mathrm{~m}, 3 \mathrm{H}), 7.17-7.15$ $(\mathrm{d}, J=7.70 \mathrm{~Hz}, 2 \mathrm{H}), 4.83-4.83(\mathrm{~d}, J=1.83 \mathrm{~Hz}, 1 . \mathrm{H}), 4.32-4.32(\mathrm{~d}, J=1.83 \mathrm{~Hz}, 1 \mathrm{H}), 2.37$ (s, $3 \mathrm{H}), 1.05(\mathrm{~s}, 10 \mathrm{H}) .{ }^{13} \mathrm{C} \mathrm{NMR}\left(126 \mathrm{MHz}, \mathrm{CDCl}_{3}\right) \delta \mathrm{ppm} \mathrm{5.3,} \mathrm{6.7,} \mathrm{21.1,} \mathrm{90.1,} \mathrm{125.2,} \mathrm{127.9,}$ 128.0, 132.0, 134.7, 135.6, 138.1. LRMS expected for $\mathrm{C}_{19} \mathrm{H}_{24} \mathrm{OSi}: 296.5$, found: 296.1.

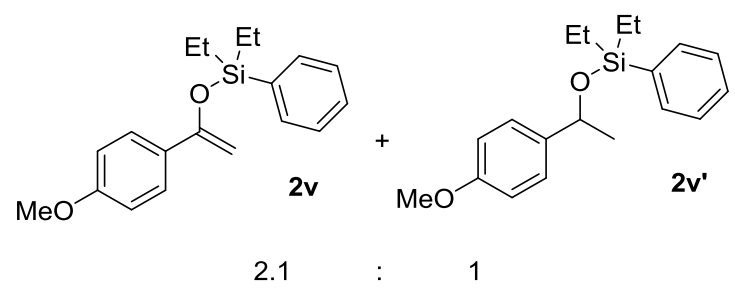

Was prepared according to the general procedure in $75 \%$ yield. Major product $\mathbf{2 v}=52 \%$. Light yellow oil. $\mathrm{R}_{\mathrm{f}}\left(20: 1-\mathrm{Hexanes}\right.$ EtOAc) $0.10 .{ }^{1} \mathrm{H} \mathrm{NMR}\left(500 \mathrm{MHz}, \mathrm{CDCl}_{3}\right)$ : $\delta \mathrm{ppm} 7.65-$ $7.63(\mathrm{dd}, J=9.54, J=7.70 \mathrm{~Hz}, 2 \mathrm{H}) 7.58-7.56(\mathrm{dd}, J=10.64, J=8.80 \mathrm{~Hz}, 2 \mathrm{H}) 7.41-7.38(\mathrm{~m}$, $3 \mathrm{H}), 6.88-6.86(\mathrm{dd}, J=11.00, J=9.17 \mathrm{~Hz}, 2 \mathrm{H}), 4.75-4.75(\mathrm{~d}, J=2.20 \mathrm{~Hz}, 1 . \mathrm{H}), 4.27-4.27(\mathrm{~d}, J$ $=1.83 \mathrm{~Hz}, 1 \mathrm{H}), 3.83(\mathrm{~s}, 3 \mathrm{H}), 1.04(\mathrm{~s}, 10 \mathrm{H}) .{ }^{13} \mathrm{C} \mathrm{NMR}\left(126 \mathrm{MHz}, \mathrm{CDCl}_{3}\right) \delta \mathrm{ppm} \mathrm{5.4,} \mathrm{6.7,} \mathrm{55.3,}$ 89.2, 113.4, 126.6, 127.8, 129.7, 133.9, 155.3, 159.7. LRMS expected for $\mathrm{C}_{19} \mathrm{H}_{24} \mathrm{O}_{2} \mathrm{Si}: 312.5$, found: 311.2 .

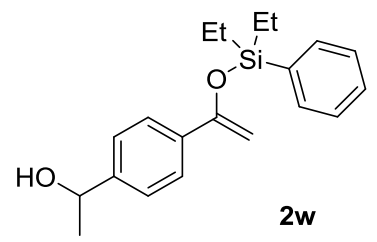

Was prepared according to the general procedure in $69 \%$ yield. Clear oil. $\mathrm{R}_{\mathrm{f}}(10: 1-\mathrm{Hexanes}$ : EtOAc) 0.06. ${ }^{1} \mathrm{H}$ NMR $\left(500 \mathrm{MHz}, \mathrm{CDCl}_{3}\right) \delta$ 7.69-7.66 (m, 4H), 7.45-7.42 (m, 3H), $7.38(\mathrm{~d}, J=$ $8.3 \mathrm{~Hz}, 2 \mathrm{H}), 4.98-4.92(\mathrm{~m}, 1 \mathrm{H}), 4.88(\mathrm{~d}, J=1.9 \mathrm{~Hz}, 1 \mathrm{H}), 4.38(\mathrm{~d}, J=1.9 \mathrm{~Hz}, 1 \mathrm{H}), 1.53(\mathrm{~d}, J=$ $6.4 \mathrm{~Hz}, 3 \mathrm{H}), 1.08(\mathrm{~s}, 10 \mathrm{H}) .{ }^{13} \mathrm{C} \mathrm{NMR}\left(126 \mathrm{MHz}, \mathrm{CDCl}_{3}\right) \delta \mathrm{ppm} \mathrm{5.4,} \mathrm{6.7,} \mathrm{25.1,} \mathrm{70.1,} \mathrm{90.8,}$ 
125.2, 125.4, 127.9, 129.7, 133.9, 135.5, 136.7, 145.9, 155.3. HRMS (EI) calcd. for $\mathrm{C}_{20} \mathrm{H}_{26} \mathrm{O}_{2} \mathrm{Si}: 326.1702$, found: 326.1702 .

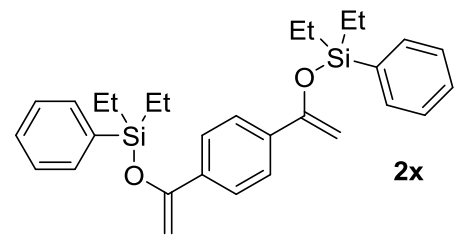

Was prepared according to the general procedure in $79 \%$ yield. Clear oil. $\mathrm{R}_{\mathrm{f}}(20: 1-$ Hexanes: EtOAc) 0.29. ${ }^{1} \mathrm{H}$ NMR (400 MHz, CDCl3) $\delta$ 7.66-7.62 (m, 8H), 7.42-7.39 (m, 6H), 4.89 (d, $J$ $=1.9 \mathrm{~Hz}, 2 \mathrm{H}), 4.37(\mathrm{~d}, J=1.9 \mathrm{~Hz}, 2 \mathrm{H}), 1.05(\mathrm{~s}, 20 \mathrm{H}) .{ }^{13} \mathrm{C} \mathrm{NMR}\left(126 \mathrm{MHz}, \mathrm{CDCl}_{3}\right) \delta \mathrm{ppm} 5.4$, 6.7, 91.0, 125.0, 127.9, 129.7, 133.9, 137.2, 145.3. HRMS (EI) calcd. for $\mathrm{C}_{30} \mathrm{H}_{38} \mathrm{O}_{2} \mathrm{Si}_{2}$ : 486.2410, found: 486.2398 .

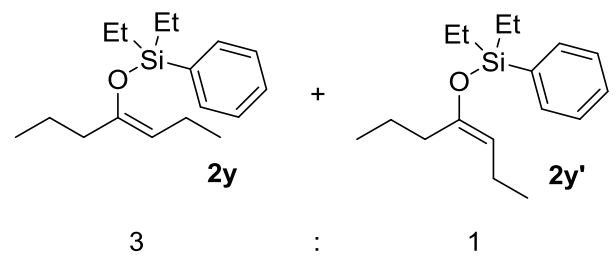

Was prepared according to the general procedure in $61 \%$ yield, Ratio $=3.0: 1.2$. Colorless oil. $\mathrm{R}_{\mathrm{f}}$ (hexanes): $0.35 .{ }^{1} \mathrm{H} \mathrm{NMR}\left(500 \mathrm{MHz}, \mathrm{CDCl}_{3}\right): \delta \mathrm{ppm}$ only olefinic protons were analyzed: $2 \mathbf{y}^{\prime}=4.63(\mathrm{t}, J=7.5 \mathrm{~Hz}, 1.2 \mathrm{H}), 2 \mathbf{y}=4.41(\mathrm{t}, J=7.0 \mathrm{~Hz}, 3 \mathrm{H}) .{ }^{13} \mathrm{C} \mathrm{NMR}\left(126 \mathrm{MHz}, \mathrm{CDCl}_{3}\right) \delta$ ppm - See below. HRMS (AP+) calcd. for $\mathrm{C}_{17} \mathrm{H}_{28} \mathrm{OSi}[\mathrm{M}]+1: 277.1988$, found: 277.1993.

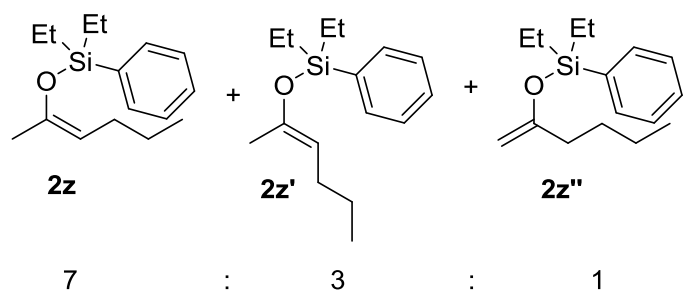

Was prepared according to the general procedure in $58 \%$ yield, Ratio $=7.0: 3.0: 1.0$. Colorless oil. $\mathrm{R}_{\mathrm{f}}$ (hexanes): $0.36 .{ }^{1} \mathrm{H}$ NMR $\left(500 \mathrm{MHz}, \mathrm{CDCl}_{3}\right): \delta \mathrm{ppm}$ only olefinic protons were analyzed: $\mathbf{2 z}$ ' $=4.66(\mathrm{t}, J=7.5 \mathrm{~Hz}, 3 \mathrm{H}), \mathbf{2 z}=4.41(\mathrm{t}, J=7.0 \mathrm{~Hz}, 7 \mathrm{H}), \mathbf{2 z} \mathbf{z}^{\prime}=4.01-4.00(\mathrm{~d}$, $2 \mathrm{H}) \cdot{ }^{13} \mathrm{C}$ NMR $\left(126 \mathrm{MHz}, \mathrm{CDCl}_{3}\right) \delta \mathrm{ppm}-$ See below. LRMS expected for $\mathrm{C}_{16} \mathrm{H}_{25} \mathrm{OSi}[\mathrm{M}]$ : 262.2, found: 262.1 . 


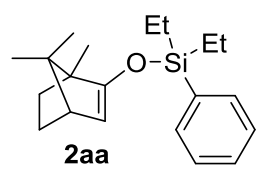

Was prepared according to the general procedure (without the cooling fan) in $40 \%$ isolated yield (2aa): Colorless oil. $\mathrm{R}_{\mathrm{f}}$ (hexanes): 0.29. ${ }^{1} \mathrm{H}$ NMR (500 $\mathrm{MHz}, \mathrm{CDCl}_{3}$ ): $\delta$ ppm 7.59-7.56 (m, 2H), 7.39-7.35 (m, 3H), $4.51(\mathrm{~d}, J=3.5 \mathrm{~Hz}, 1 \mathrm{H}), 2.16-2.12(\mathrm{~m}, 1 \mathrm{H}), 1.03-0.92(\mathrm{~m}, 23 \mathrm{H})$. ${ }^{13} \mathrm{C} \mathrm{NMR}\left(126 \mathrm{MHz}, \mathrm{CDCl}_{3}\right) \delta \mathrm{ppm} 5.2,6.6,10.0,19.7,20.1,27.3,31.3,45.3,49.5,53.6,54.7$, 103.0, 127.6, 127.7, 129.5, 133.9, 160.1. HRMS (AP+) calcd. for $\mathrm{C}_{20} \mathrm{H}_{30} \mathrm{OSi}[\mathrm{M}]+1: 315.2144$, found: 315.2138 .

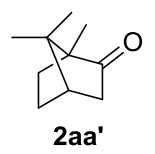

Was prepared according to the general procedure (without the cooling fan) in 55\% isolated yield (2aa'): Colorless oil. NMR and GC data correspond to commercially available compound.

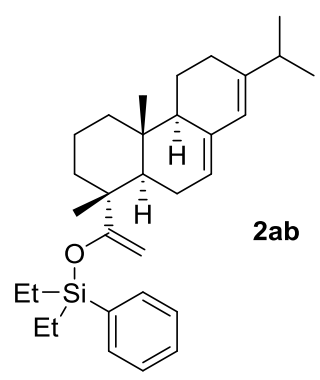

Was prepared according to the general procedure in $58 \%$ yield. Clear and Colorless oil. $\mathrm{R}_{\mathrm{f}}$ (Hexanes) 0.20. ${ }^{1} \mathrm{H} \mathrm{NMR}\left(500 \mathrm{MHz}, \mathrm{CDCl}_{3}\right): \delta \mathrm{ppm}$ 7.57-7.55 (d, $\left.J=6.24 \mathrm{~Hz}, 2 \mathrm{H}\right), 7.39-7.34$ (q, 3H), 5.79 (s, 1H), 5.39 (br s, 1H), 4.08-4.08 (d, J=1.47 Hz, 1H), 3.99-3.99 (d, J = 1.47 Hz, $1 \mathrm{H}), 2.25-2.21(\mathrm{~m}, 1 \mathrm{H}), 1.81-2.10(\mathrm{~m}, 8 \mathrm{H}), 1.64-1.53(\mathrm{~m}, 2 \mathrm{H}), 1.33-1.36(\mathrm{~d}, J=12.10 \mathrm{~Hz}, 1 \mathrm{H})$, 1.27-1.20 (m, 2H), $1.11(\mathrm{~s}, 3 \mathrm{H}), 1.04-0.88(\mathrm{~m}, 16 \mathrm{H}), 0.84(\mathrm{~s}, 3 \mathrm{H}), 0.72-0.61(\mathrm{~m}, 1 \mathrm{H}) .{ }^{13} \mathrm{C} \mathrm{NMR}$ $\left(126 \mathrm{MHz}, \mathrm{CDCl}_{3}\right) \delta$ ppm 5.4, 5.5, 6.7, 6.8, 14.2, 18.5, 18.6, 20.8, 21.4, 24.7, 27.4, 34.6, 34.9, $36.9,38.9,43.3,51.4,88.8,121.8,122.8,127.7,133.9,135.3,136.0,144.7,165.3$. HRMS (ESI) calcd. for $\mathrm{C}_{31} \mathrm{H}_{47} \mathrm{OSi}[\mathrm{M}]+1: 463.3396$, found: 463.3388 . 


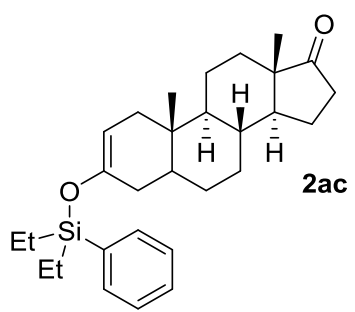

Was prepared according to the general procedure in $75 \%$ yield. Light yellow oil. $R_{\mathrm{f}}(9: 1-$ Hexanes: EtOAc): 0.27. ${ }^{1} \mathrm{H}$ NMR (500 MHz, $\left.\mathrm{CDCl}_{3}\right): \delta$ ppm 7.59-7.56 (m, 2H), 7.39-7.34 (m, $3 \mathrm{H}), 4.74-4.72(\mathrm{~d}, J=5.84 \mathrm{~Hz}, 1 \mathrm{H}), 2.47-2.40(\mathrm{dd}, J=9.17, J=19.27,1 \mathrm{H}), 2.10-2.01(\mathrm{~m}, 1 \mathrm{H})$, 1.94-1.86 (m, 2H), 1.83-1.73 (m, 3H), 1.71-1.56 (m, 2H), 1.55-1.35 (m, 4H), 1.34-1.17 (m, $5 \mathrm{H}), 1.01-0.91(\mathrm{~m}, 10 \mathrm{H}), 0.85(\mathrm{~s}, 3 \mathrm{H}) 0.83-0.82(\mathrm{~d}, J=4.38,2 \mathrm{H}), 0.71(\mathrm{~s}, 3 \mathrm{H}),{ }^{13} \mathrm{C}$ NMR $(126$ $\left.\mathrm{MHz}, \mathrm{CDCl}_{3}\right) \delta$ ppm 5.5, 5.5, 6.6, 11.6, 13.7, 13.8, 20.4, 21.8, 28.2, 30.6, 31.6, 34.4, 34.6, 35.1, 35.8, 38.4, 42.0, 47.7, 51.4, 51.5, 54.5, 53.7, 102.7, 127.7, 129.5, 133.9, 136.2, 148.8, 221.3. HRMS (ESI) calcd. for $\mathrm{C}_{29} \mathrm{H}_{43} \mathrm{O}_{2} \mathrm{Si}[\mathrm{M}]+1: 451.3032$, found: 451.3026 . 


\section{Mechanistic Studies}

\section{Proposed Mechanism:}

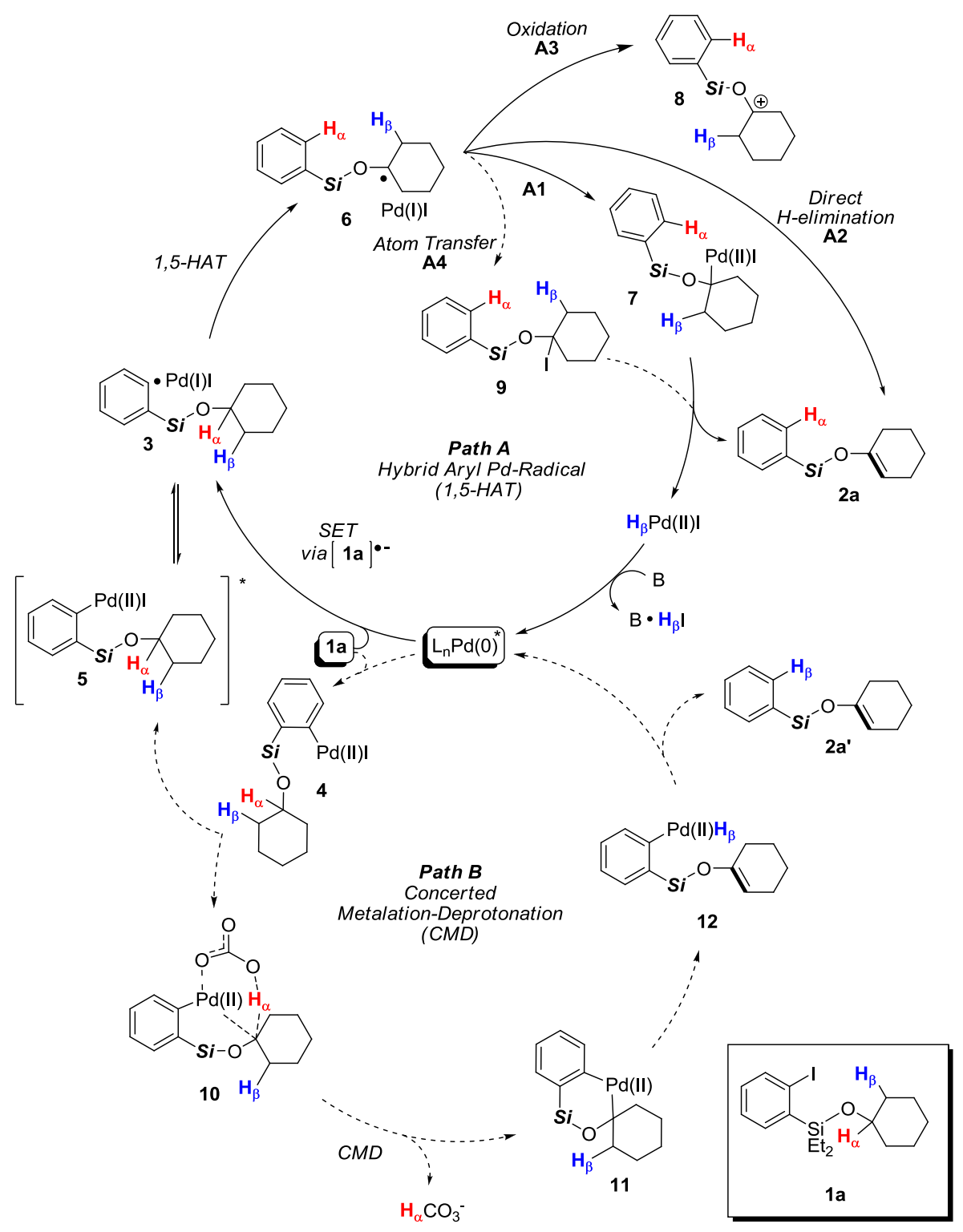

Apparently, these pathways (Path A vs B) can easily be distinguished by the results of isotope labeling studies of 1a- $d\left(\mathrm{H}_{\alpha}=\mathrm{D}, \mathrm{H}_{\beta}=\mathrm{H}\right)$. Indeed, if Path A operates, desaturation of $\mathbf{1 a}-d$ will lead to the deuterium-incorporated product $\mathbf{2 a}-d$. In contrast, if hydrogen is transferred (2a') then a CMD (Path B) pathway is operative. 


\section{Isotope Labeling Studies: To distinguish 1,5-HAT vs CMD Mechanism}

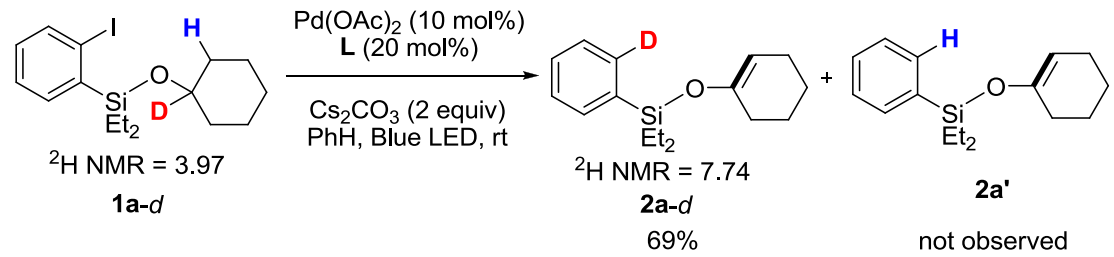

The performed experiments revealed that desaturation of 1a- $d$ produced $\mathbf{2 a}-d$ exclusively with $\geq 98 \%$ deuterium incorporation at the aryl silane moiety, thus, strongly supporting the hybrid Pd-radical Path A.

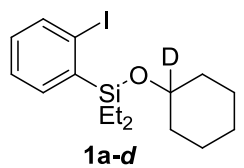

Was prepared according to the general procedure A (S4) in 55\% yield. >98\% D incorporation. Colorless oil. $\mathrm{R}_{\mathrm{f}}$ (hexanes): 0.20. ${ }^{1} \mathrm{H}$ NMR (500 MHz, $\left.\mathrm{CDCl}_{3}\right): \delta$ ppm 7.85-7.84 $(\mathrm{d}, J=8.07$ $\mathrm{Hz}, 1 \mathrm{H}), 7.64-7.63(\mathrm{dd}, J=7.34 \mathrm{~Hz}, J=1.47 \mathrm{~Hz}, 1 \mathrm{H}), 7.36-7.33$ (t, 1H), 7.05-7.02 (td, $J=8.07$ $\mathrm{Hz}, J=1.83 \mathrm{~Hz}, 1 \mathrm{H}), 1.87-1.85(\mathrm{~m}, 2 \mathrm{H}), 1.76-1.73$ (m, 2H), 1.52-1.43 (m, 3H), 1.31-1.22 (m, $3 \mathrm{H}), 1.14-0.98(\mathrm{~m}, 4 \mathrm{H}), 0.96-0.94(\mathrm{~m}, 6 \mathrm{H}) .{ }^{13} \mathrm{C} \mathrm{NMR}\left(126 \mathrm{MHz}, \mathrm{CDCl}_{3}\right) \delta \mathrm{ppm} 5.4,7.0,24.2$, 25.7, 35.8, 102.7, 126.8, 130.9, 138.1, 139.7, 142.8. ${ }^{2} \mathrm{H}$ NMR (77 MHz, $\left.\mathrm{CCl}_{4}\right) \delta \mathrm{ppm} 3.97$. HRMS (EI+) calcd. for $\mathrm{C}_{16} \mathrm{H}_{24} \mathrm{D}_{1} \mathrm{IOSi}[\mathrm{M}]$ : 389.0782, found: 375.0794 .

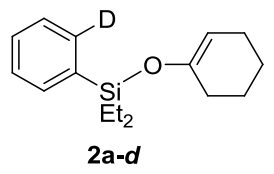

Was prepared according to the general procedure (S19) for $60 \mathrm{~h}$ in $69 \%$ yield. Clear and colorless oil. $\mathrm{R}_{\mathrm{f}}$ (hexanes): 0.34. ${ }^{1} \mathrm{H} \mathrm{NMR}\left(500 \mathrm{MHz}, \mathrm{CDCl}_{3}\right): \delta \mathrm{ppm}$ 7.53-7.51 (m, 1H), 7.33$7.31(\mathrm{~m}, 3 \mathrm{H}), 4.77-4.76(\mathrm{t}, 1 \mathrm{H}), 2.03-2.01$ (m, 2H), 1.99-1.96 (m, 4H), 1.67-1.63 (m, 2H), 1.531.49 (m, 2H), 1.03-0.99 (m, 6H), 0.88-0.92 (m, 4H). ${ }^{2} \mathrm{H}$ NMR (77 MHz, $\left.\mathrm{CCl}_{4}\right) \delta$ ppm 7.75. HRMS (AP+) calcd. for $\mathrm{C}_{16} \mathrm{H}_{24} \mathrm{DOSi}[\mathrm{M}]+1: 262.1737$, found: 262.1746. 


\section{Radical Scavengers Experiments:}

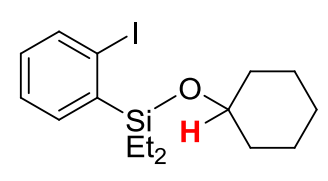

$1 a$

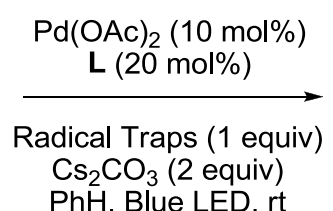

PhH, Blue LED, $\mathrm{rt}$

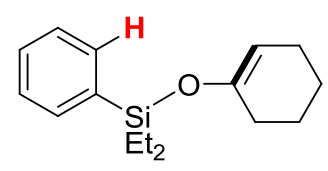

$2 \mathbf{a}$

\begin{tabular}{cc} 
Radical Traps & NMR Yield, \% \\
\hline None & 79 \\
BHT & 65 \\
Galvinyloxy & NR \\
TEMPO & NR
\end{tabular}

\section{Radical Clock Experiment:}

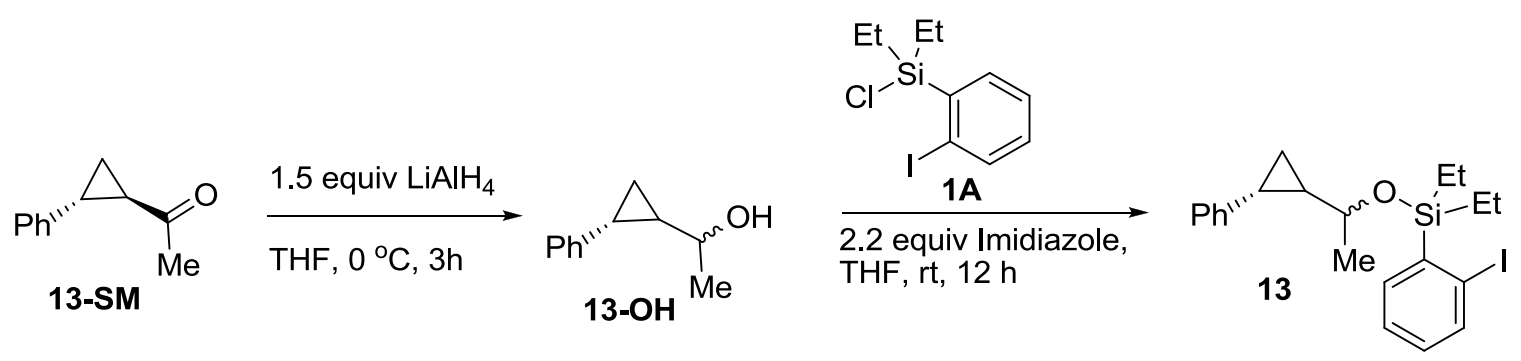

To a suspension of $\mathrm{LiAlH}_{4}(1.5$ equiv, $0.43 \mathrm{~g}, 11.4 \mathrm{mmol})$ in THF $(20 \mathrm{~mL})$ at $0{ }^{\circ} \mathrm{C}$ under argon, 13-SM ${ }^{4}$ (1 equiv, $\left.1.22 \mathrm{~g}, 7.61 \mathrm{mmol}\right)$ in THF $(10 \mathrm{~mL})$ was added slowly. The resulting mixture was stirred at $0{ }^{\circ} \mathrm{C}$ for $3 \mathrm{~h}$. A saturated ammonium chloride solution was added and the aqueous phase was extracted with ether $(3 \times 50 \mathrm{~mL})$. The organic phase was dried over $\mathrm{Na}_{2} \mathrm{SO}_{4}$, filtered and concentrated. The compound was purified by flash (20:1 Hex:EtOAc) to give 13-OH as a clear and colorless oil (d.r. = 1.5:1 (GC) , 97\%, $1.2 \mathrm{~g})$. Next, 13 was prepared using general procedure A (S4) using 13-OH.<smiles>CCOc1ccccc1I</smiles>

Was prepared according to the general procedure A (S4) using 13-OH in $40 \%$ yield. Diastereomeric Ratio = 1.5:1 (GC). Clear and Colorless oil. $\mathrm{R}_{\mathrm{f}}(50: 1$ Hexanes : Ethyl Acetate): 0.3. ${ }^{1} \mathrm{H}$ NMR $\left(500 \mathrm{MHz}, \mathrm{CDCl}_{3}\right): \delta \mathrm{ppm} 7.88-7.82(\mathrm{dd}, J=7.07 \mathrm{~Hz}, J=8.07 \mathrm{~Hz}, 1 \mathrm{H}), 7.65-$ $7.59(\mathrm{dd}, J=7.34 \mathrm{~Hz}, J=7.34 \mathrm{~Hz}, 1 \mathrm{H}), 7.37-7.24(\mathrm{~m}, 3 \mathrm{H}), 7.17-7.14(\mathrm{~m}, 1 \mathrm{H}), 7.07-7.00$ (m, 
3H), 3.71-3.67 (m, 1H), 1.91-1.79 (m, 1H), 1.39-1.36 (m, 3H), 1.18-0.88 (m, 13H). ${ }^{13} \mathrm{C}$ NMR $\left(126 \mathrm{MHz}, \mathrm{CDCl}_{3}\right) \delta$ ppm 5.3, 5.4, 5.5, 5.6, 6.9, 7.0, 13.4, 14.5, 20.6, 21.7, 23.7, 23.9, 30.8, 31.0, 71.9, 72.0, 102.7, 102.8, 125.3, 125.4, 125.8, 125.9, 126.9, 128.2, 128.3, 130.9, 131.0, 138.0, 138.2, 139.7, 139.8, 142.5, 142.6, 143.0, 143.2. HRMS (EI) calcd. for $\mathrm{C}_{21} \mathrm{H}_{27} \mathrm{IOSi}[\mathrm{M}]:$ : 450.0876, found: 450.0880 .

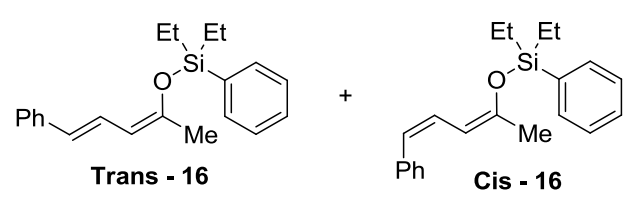

Was prepared according to the general procedure (S19) in 53\% NMR yield of Trans-16 and $25 \%$ NMR yield of Cis-16, Total Yield $=78 \%$. Crude ${ }^{1} \mathrm{H}$ NMR (500 MHz, $\left.\mathrm{C}_{6} \mathrm{D}_{6}\right)$ Trans 16 (only Olefinic protons and the methyl group are reported): $\delta \mathrm{ppm} 7.61-7.55$ (dd, $J=10.6 \mathrm{~Hz}, J$ $=15.7 \mathrm{~Hz}, 1 \mathrm{H}), 6.51-6.48(\mathrm{~d}, J=16.4 \mathrm{~Hz}, 1 \mathrm{H}), 5.49-5.47(\mathrm{~d}, J=10.6 \mathrm{~Hz}, 1 \mathrm{H}), 1.79$ (s, 3H). Crude ${ }^{1} \mathrm{H}$ NMR (500 MHz, $\mathrm{C}_{6} \mathrm{D}_{6}$ ) ) Cis-16 (only Olefinic protons and the methyl group are reported): $\delta$ ppm 6.95-6.90 (dd, $J=11.0 \mathrm{~Hz}, J=15.4 \mathrm{~Hz}, 1 \mathrm{H}), 5.95-5.93(\mathrm{~d}, J=11.0 \mathrm{~Hz}, 1 \mathrm{H})$, 1.95 (s, 3H). HRMS (EI) calcd. for $\mathrm{C}_{21} \mathrm{H}_{26} \mathrm{OSi}[\mathrm{M}]: 322.1753$, found: 322.1749 .

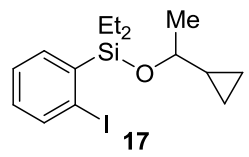

Was prepared according to the general procedure $\mathbf{A}$ (S4) in $23 \%$ yield. Colorless oil. $\mathrm{R}_{\mathrm{f}}$ (hexanes): 0.12. ${ }^{1} \mathrm{H}$ NMR $\left(400 \mathrm{MHz}, \mathrm{CDCl}_{3}\right): \delta \mathrm{ppm} 7.85-7.83(\mathrm{~d}, J=7.89 \mathrm{~Hz}, 1 \mathrm{H}), 7.64-7.62$ $(\mathrm{dd}, J=7.31 \mathrm{~Hz}, J=1.75,1 \mathrm{H}), 7.36-7.32(\mathrm{t}, 1 \mathrm{H}), 7.05-7.01(\mathrm{td}, J=7.60 \mathrm{~Hz}, J=2.05,1 \mathrm{H})$, 3.35-3.32 (q, 1H), 1.32-1.28 (d, 3H), 1.14-0.98 (m, 4H), 0.96-0.94 (m, 7H), 0.46-0.42 (m, 2H), 0.24-0.19 (m, 1H), 0.16-0.12 (m, 1H). ${ }^{13} \mathrm{C}$ NMR $\left(100 \mathrm{MHz}, \mathrm{CDCl}_{3}\right) \delta \mathrm{ppm} 2.2,4.2,5.4,5.5$, 6.9, 19.1, 23.8, 73.6, 107.0, 126.8, 130.9, 138.1, 139.7, 148.6. HRMS (AP+) calcd. for $\mathrm{C}_{15} \mathrm{H}_{24} \mathrm{IOSi}[\mathrm{M}]+1: 375.0641$, found: 375.0651 .

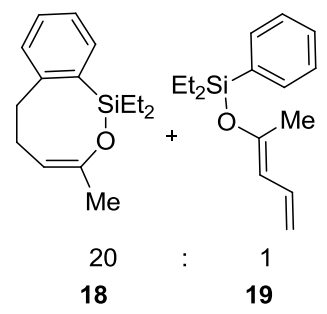


Was prepared according to the general procedure (S19) in $40 \%$ NMR yield. Crude. ${ }^{1} \mathrm{H}$ NMR (400 MHz, $\left.\mathrm{C}_{6} \mathrm{D}_{3}\right): \delta$ ppm major product: 7.25-7.20 (m, $\left.4 \mathrm{H}\right), 4.28-4.24(\mathrm{~m}, 1 \mathrm{H}), 3.35-3.32(\mathrm{q}$, $1 \mathrm{H}), 2.84-2.81(\mathrm{t}, 2 \mathrm{H}), 2.60-2.56(\mathrm{~m}, 2 \mathrm{H}), 1.54(\mathrm{~s}, 3 \mathrm{H}), 1.05-0.88(\mathrm{~m}, 10 \mathrm{H})$. LRMS expected for $\mathrm{C}_{15} \mathrm{H}_{22} \mathrm{OSi}$ : 246.3, found: 246.1 . 


\section{Stoichiometric Studies}

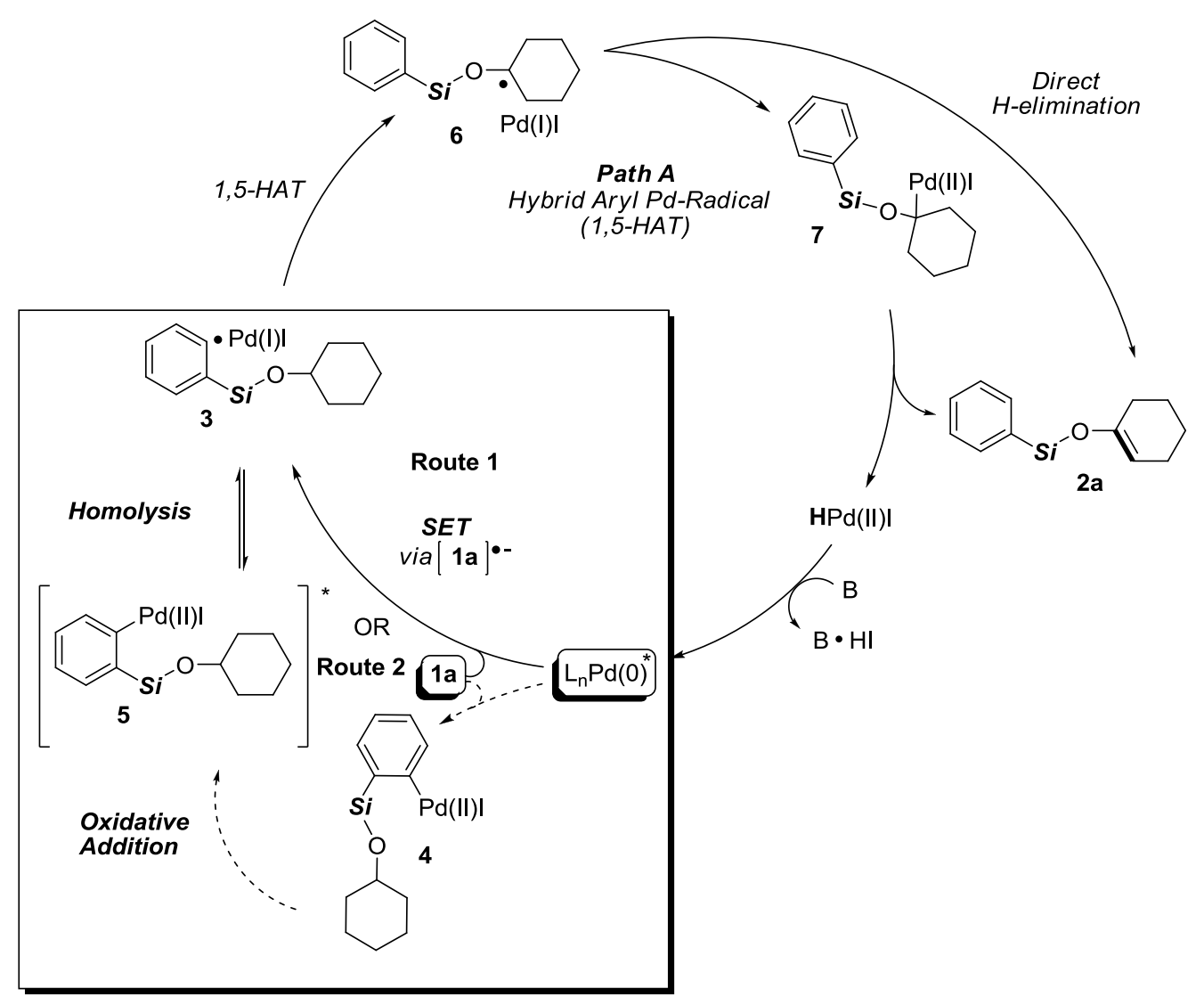

In order to determine whether the formation of the aryl hybrid Pd-radical intermediate $\mathbf{3}$ is generated via SET (Route 1) or via Oxidative Addition and subsequent photoinduced homolysis (Route 2), stoichiometric studies with $\operatorname{Pd}(0)$ and 1 a were conducted (eq 1).

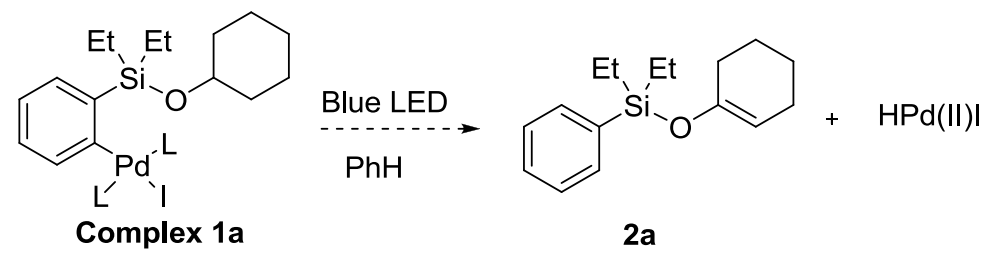

Based on equation 1, if complex 1a under visible light photoirradiation produces 2a, then Route 2 is the likely pathway. If no reaction occurs, then a SET process (Route 1) is operative. 
Table S3: Oxidative Addition of 1a with Pd(0) Precursors:

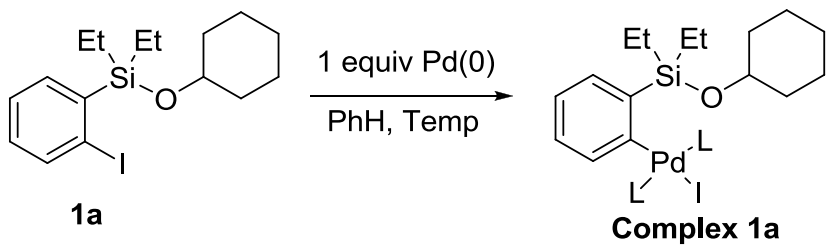

\begin{tabular}{|c|c|c|c|}
\hline Entry & $\mathrm{Pd}(0)$ & Temp, ${ }^{\circ} \mathrm{C}$ & GC \\
\hline 1 & $\mathrm{Pd}\left(\mathrm{PPh}_{3}\right)_{4}$ & $\mathrm{rt}$ & NR, 1a stays \\
\hline 2 & $\mathrm{Pd}\left(\mathrm{PPh}_{3}\right)_{4}$ & 85 & NR, 1a stays \\
\hline 3 & $\mathrm{Pd}\left(\mathrm{PPh}_{3}\right)_{4}$ & 150 & NR, 1a stays \\
\hline 4 & $\mathrm{Pd}(\mathrm{dba})_{2} / \mathbf{L}$ & $\mathrm{rt}$ & NR, 1a stays \\
\hline 5 & $\mathrm{Pd}(\mathrm{dba})_{2} / \mathbf{L}$ & 85 & NR, 1a stays \\
\hline 6 & $\mathrm{Pd}(\mathrm{dba})_{2} / \mathrm{dppf}$ & $\mathrm{rt}$ & $\mathrm{NR}, \mathbf{1 a}$ stays \\
\hline 7 & $\mathrm{Pd}(\mathrm{dba})_{2} / \mathrm{dppf}$ & 85 & NR, 1a stays \\
\hline 8 & $\mathrm{Pd}(\mathrm{OAc})_{2} / \mathbf{L}$ & $\mathrm{rt}$ & NR, 1a stays \\
\hline 9 & $\mathrm{Pd}(\mathrm{OAc})_{2} / \mathbf{L}$ & 85 & $\begin{array}{c}\text { NR, 1a stays } \\
\text { Complex 1a was not } \\
\text { observed by NMR }\end{array}$ \\
\hline 10 & $\mathrm{Pd}(\mathrm{OAc})_{2} / \mathbf{L}$ & 120 & \\
& & &
\end{tabular}

As shown in Table S3, all attempts on the synthesis of Complex 1a failed, probably due to the presence of the bulky silyl group at the ortho position to the iodide. Thus, the likelihood of Route 2 was questioned. Accordingly, the SET-based Route 1 appears to be more plausible. 
Table S4: Atom-Transfer of 1a with stoichiometric Pd(0):<smiles>CC[Si](CC)(OC1CCCCC1)c1ccccc1I</smiles>

$1 \mathrm{a}$

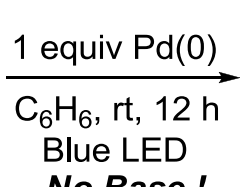

No Base !

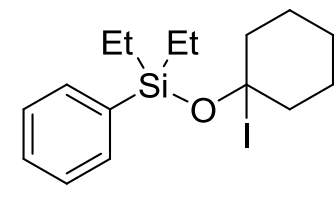

9<smiles>CC[Si](CC)(OC1=CCCCC1)c1ccccc1</smiles>

$2 a$

\begin{tabular}{|c|c|c|c|}
\hline Entry & $\mathrm{Pd}(0)$ & $\mathbf{9}(\mathrm{GC} / \mathrm{NMR})$ & 2a (GC Yield) \\
\hline 1 & $\mathrm{Pd}^{\left(\mathrm{PPh}_{3}\right)_{4}}$ & Not Observed & $68 \%$ \\
\hline 2 & $\mathrm{Pd}(\mathrm{OAc})_{2} / \mathbf{L}$ & Not Observed & $70 \%$ \\
\hline 3 & $10 \% \mathrm{Pd}(\mathrm{OAc})_{2}$ & Not Observed & NR \\
& $20 \% \mathbf{L}$ & & \\
& & & \\
\hline
\end{tabular}

In order to determine if our protocol proceeds via an Atom-Transfer process, stoichiometric studies with $\operatorname{Pd}(0)$ and 1a in the absence of base were conducted (Table S4, entries 1-2). As shown above, $\mathbf{9}$ was not observed by GC/NMR analysis only the desaturation product $\mathbf{2 a}$ was obtained. Therefore, an Atom-Transfer mechanism may not be operative (Scheme 2, Path A4 (Manuscript), or see S31). Also, using a catalytic amount of catalyst and ligand led to no reaction (Table $\mathrm{S} 4$, entries 3 ). 


\section{NMR Spectral Data}

${ }^{1} \mathrm{H}$ Spectrum of $\mathbf{1 A}$

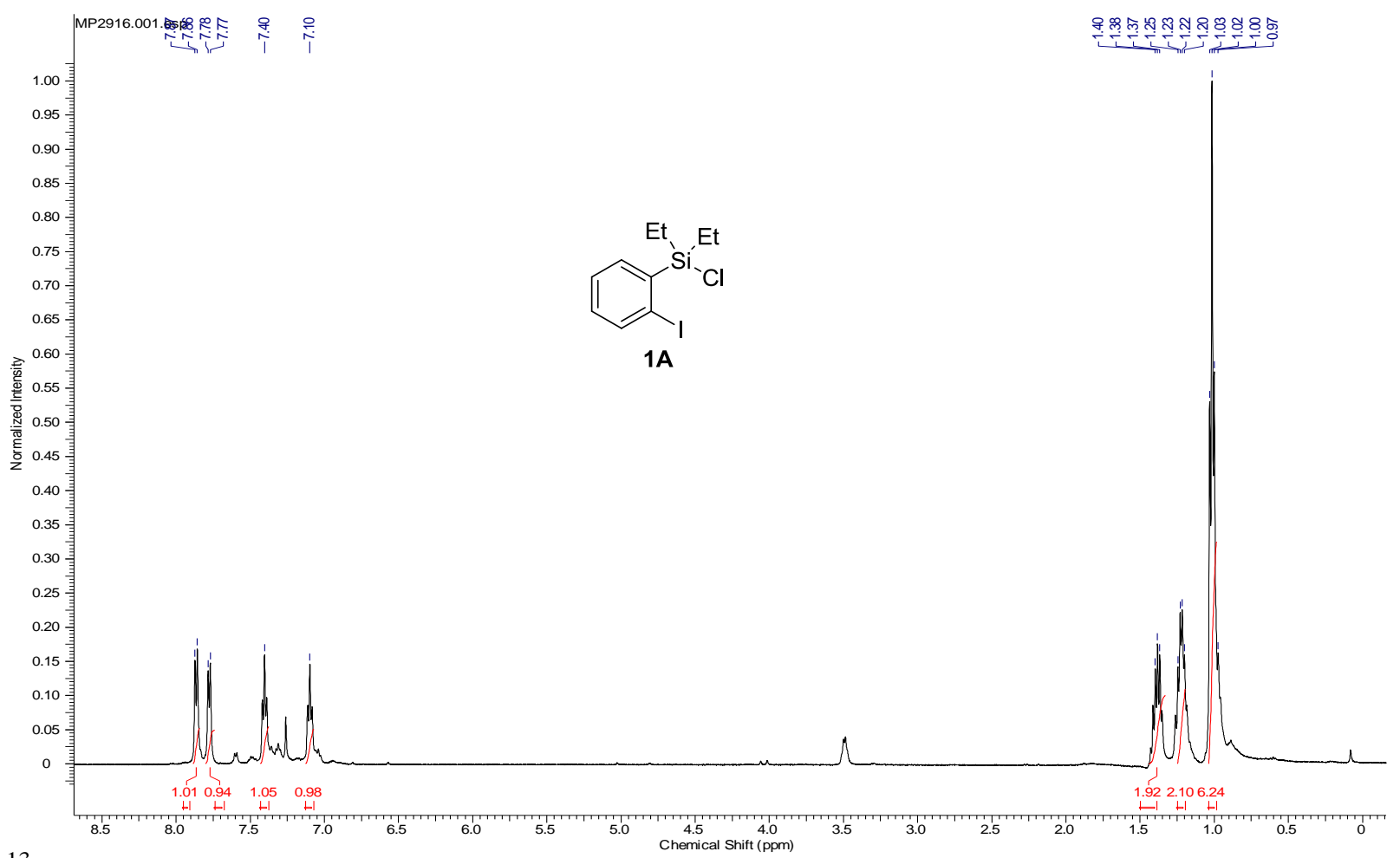

${ }^{13} \mathrm{C}$ Spectrum of $\mathbf{1 A}$

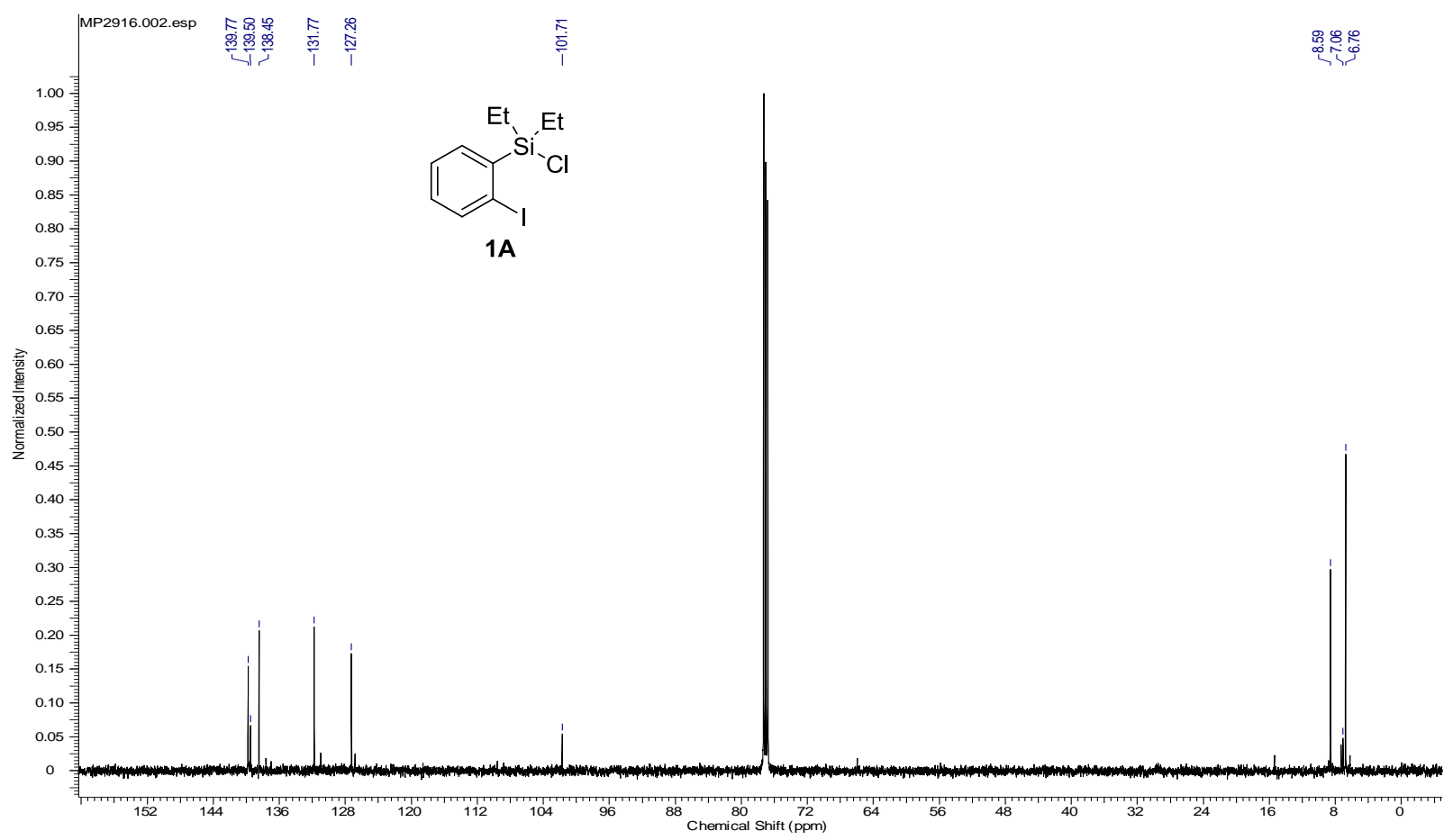




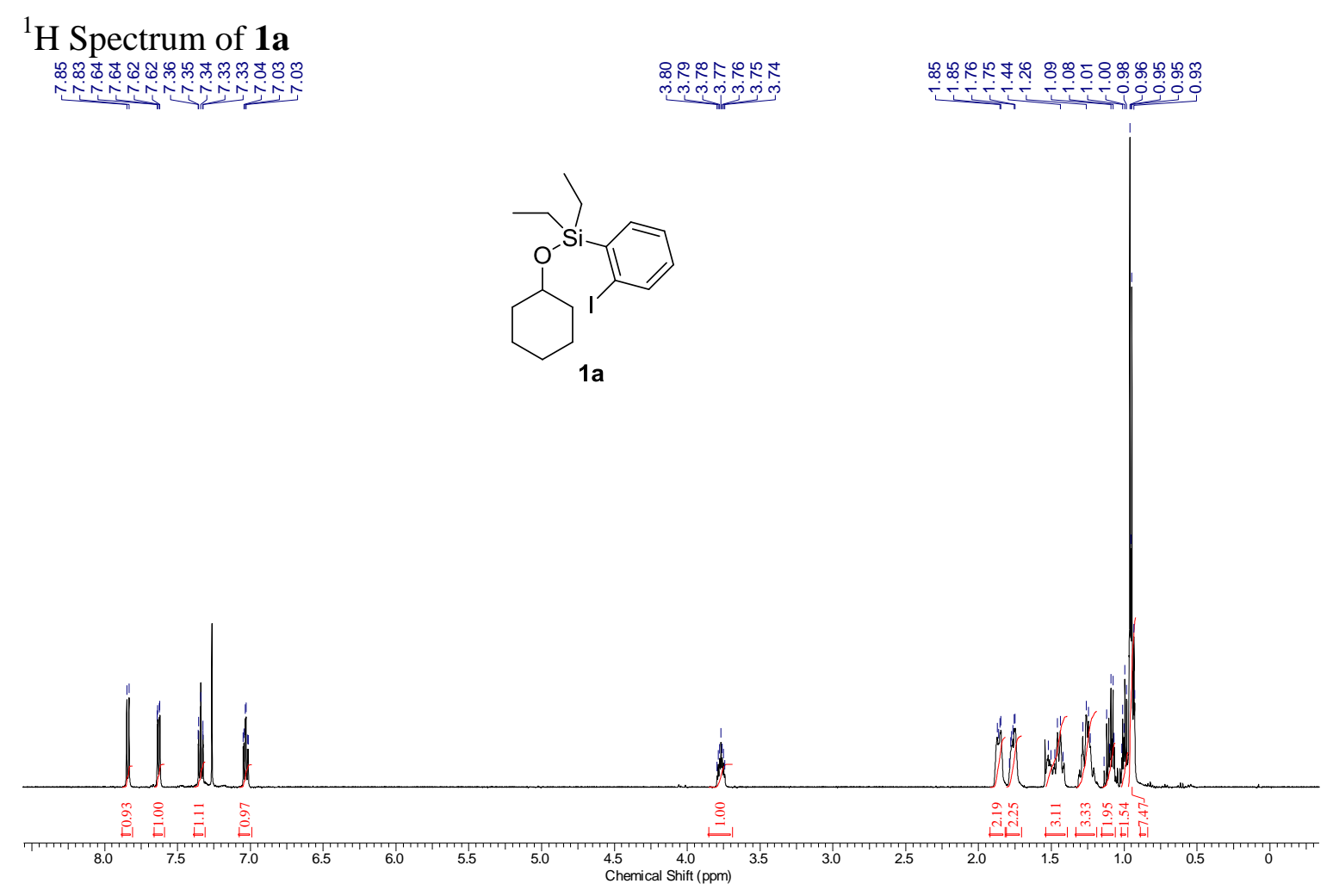

\section{${ }^{13} \mathrm{C}$ Spectrum of $\mathbf{1 a}$}

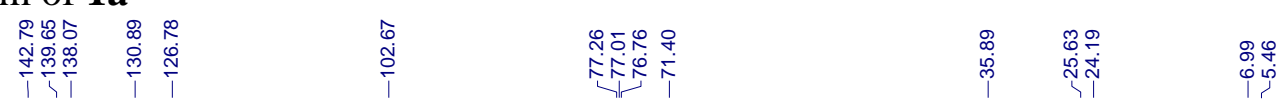

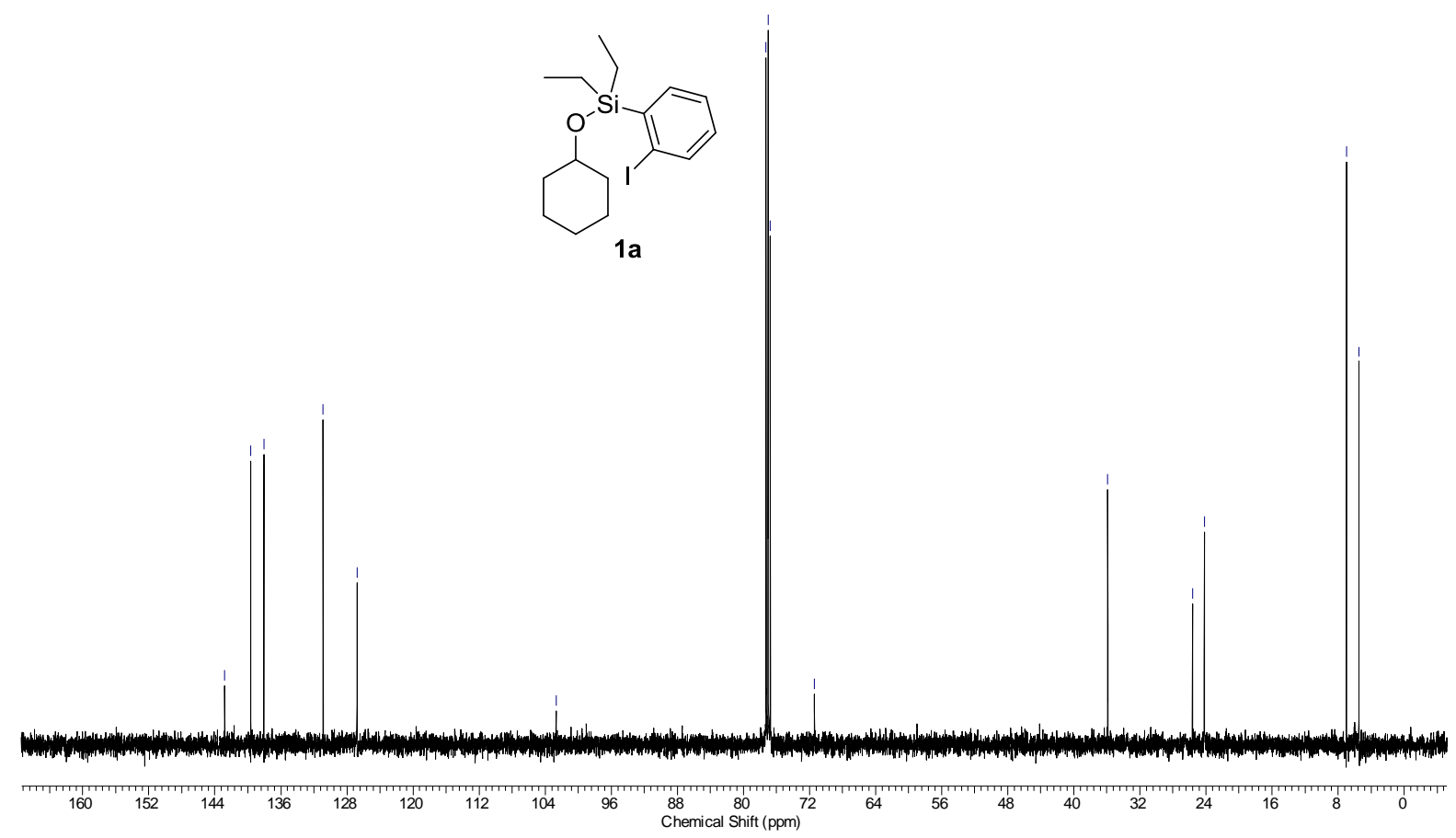




\section{${ }^{1} \mathrm{H}$ Spectrum of $\mathbf{1 b}$}

$$
\text { | }
$$

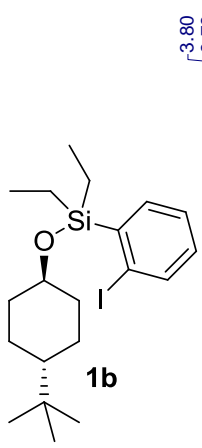

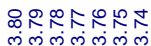

लंखिल

ஹூ్య
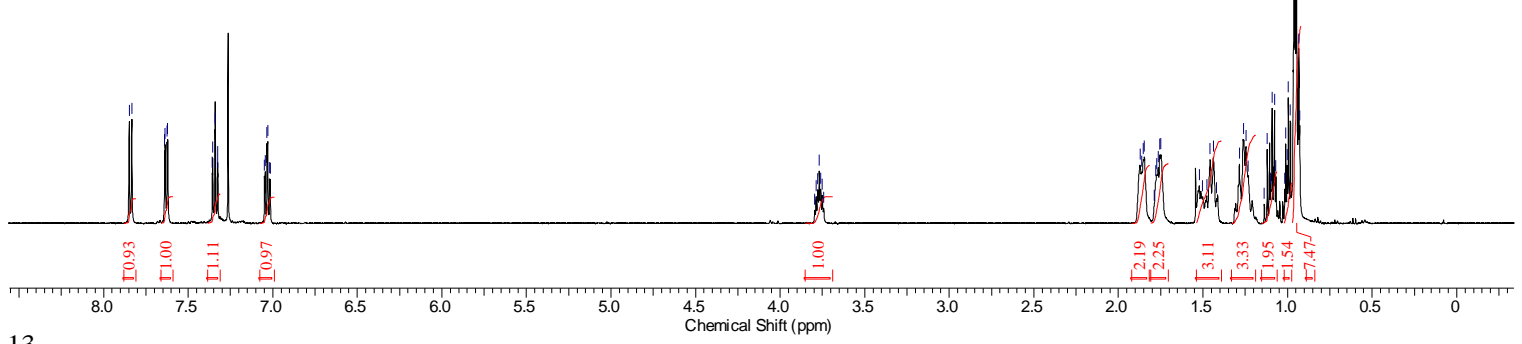

${ }^{13} \mathrm{C}$ Spectrum of $\mathbf{1 b}$

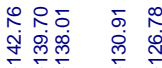

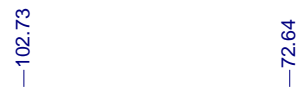

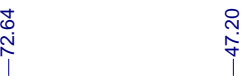

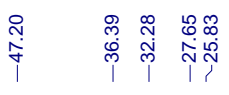

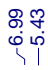
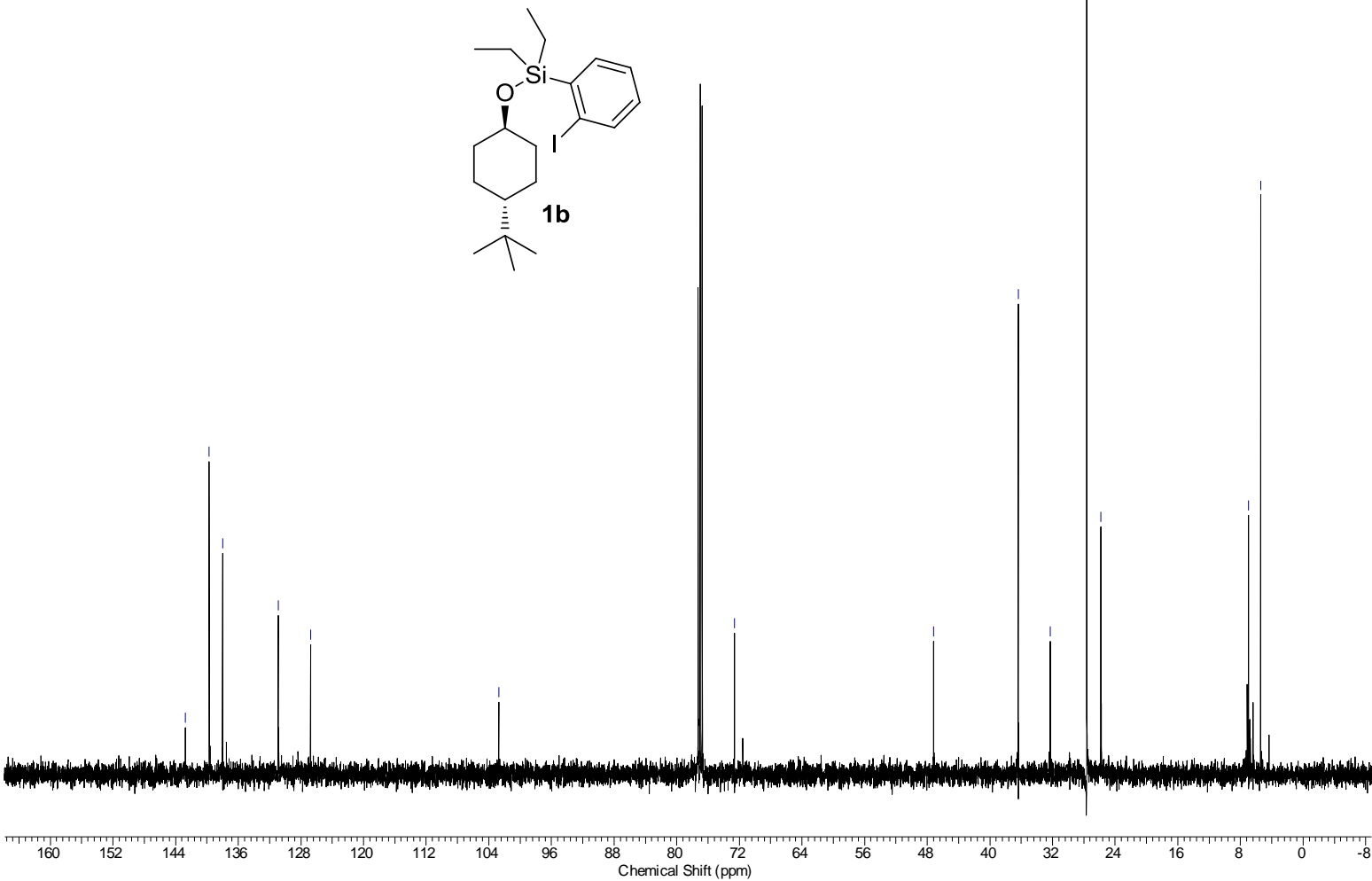


\section{${ }^{1} \mathrm{H}$ Spectrum of $\mathbf{1 c}$}

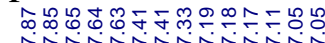

hinininitinitis

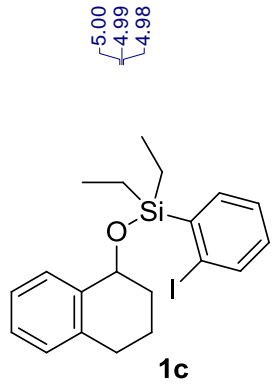

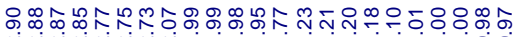
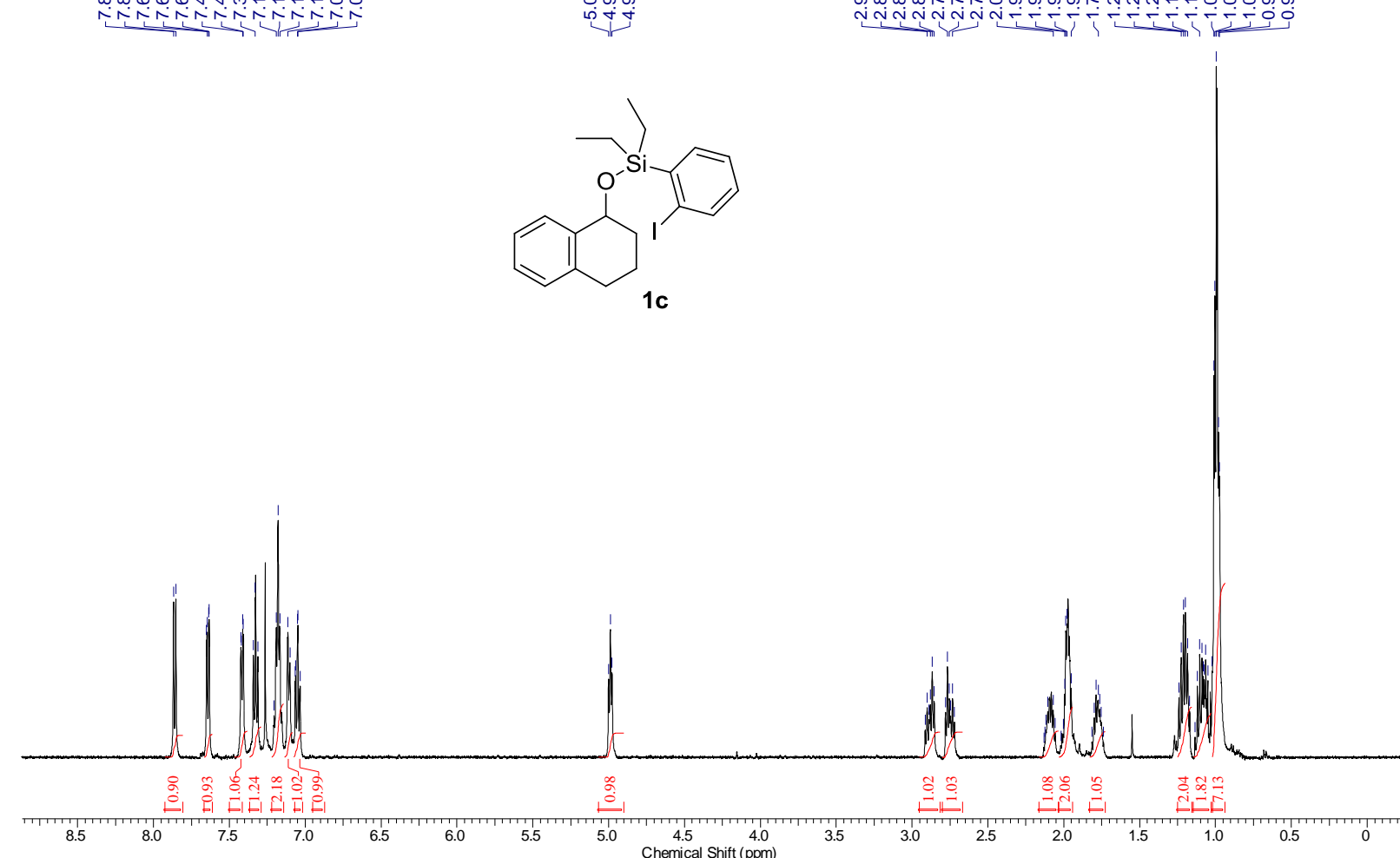

${ }^{13} \mathrm{C}$ Spectrum of $1 \mathrm{c}$

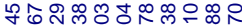

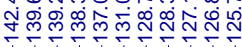

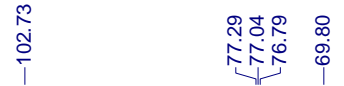

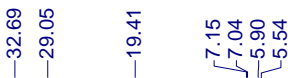<smiles>CC[Si](CC)(OC1CCCc2ccccc21)c1ccccc1I</smiles>

$1 \mathrm{c}$

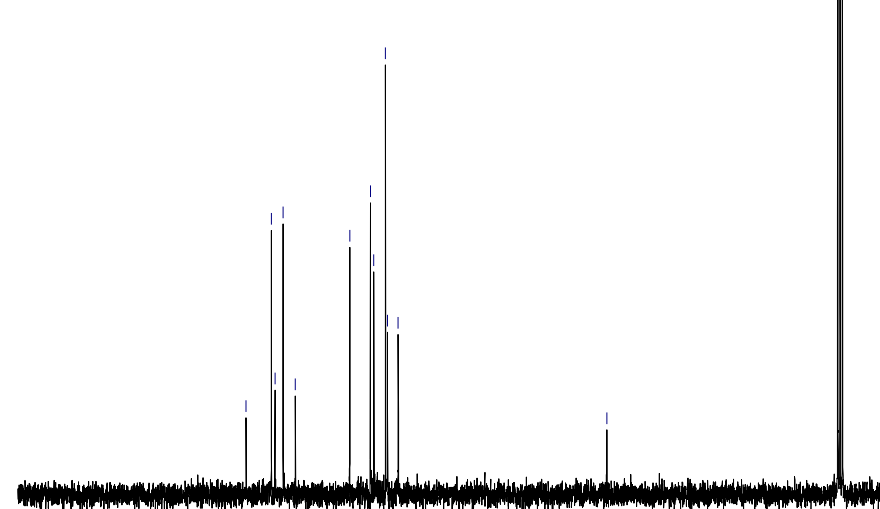

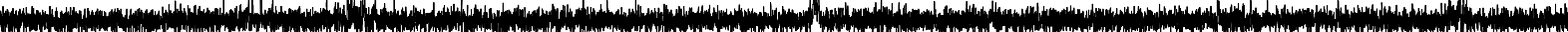

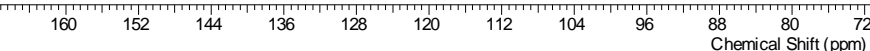


${ }^{1} \mathrm{H}$ Spectrum of $\mathbf{1 d}$

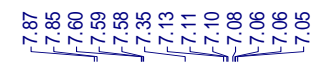
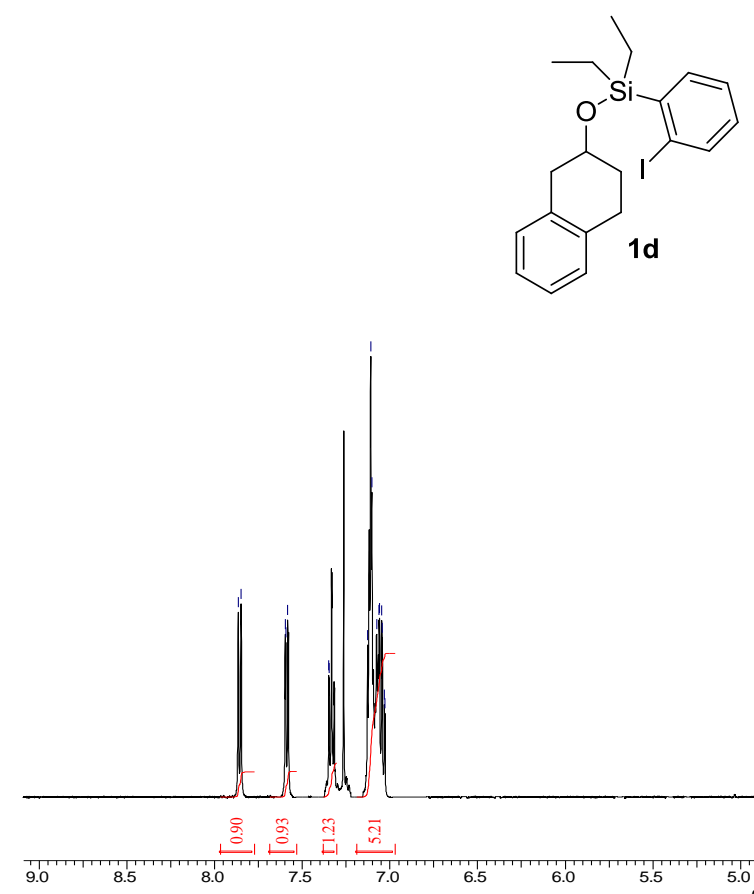

${ }^{13} \mathrm{C}$ Spectrum of $\mathbf{1 d}$

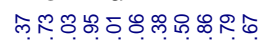

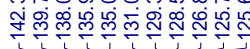
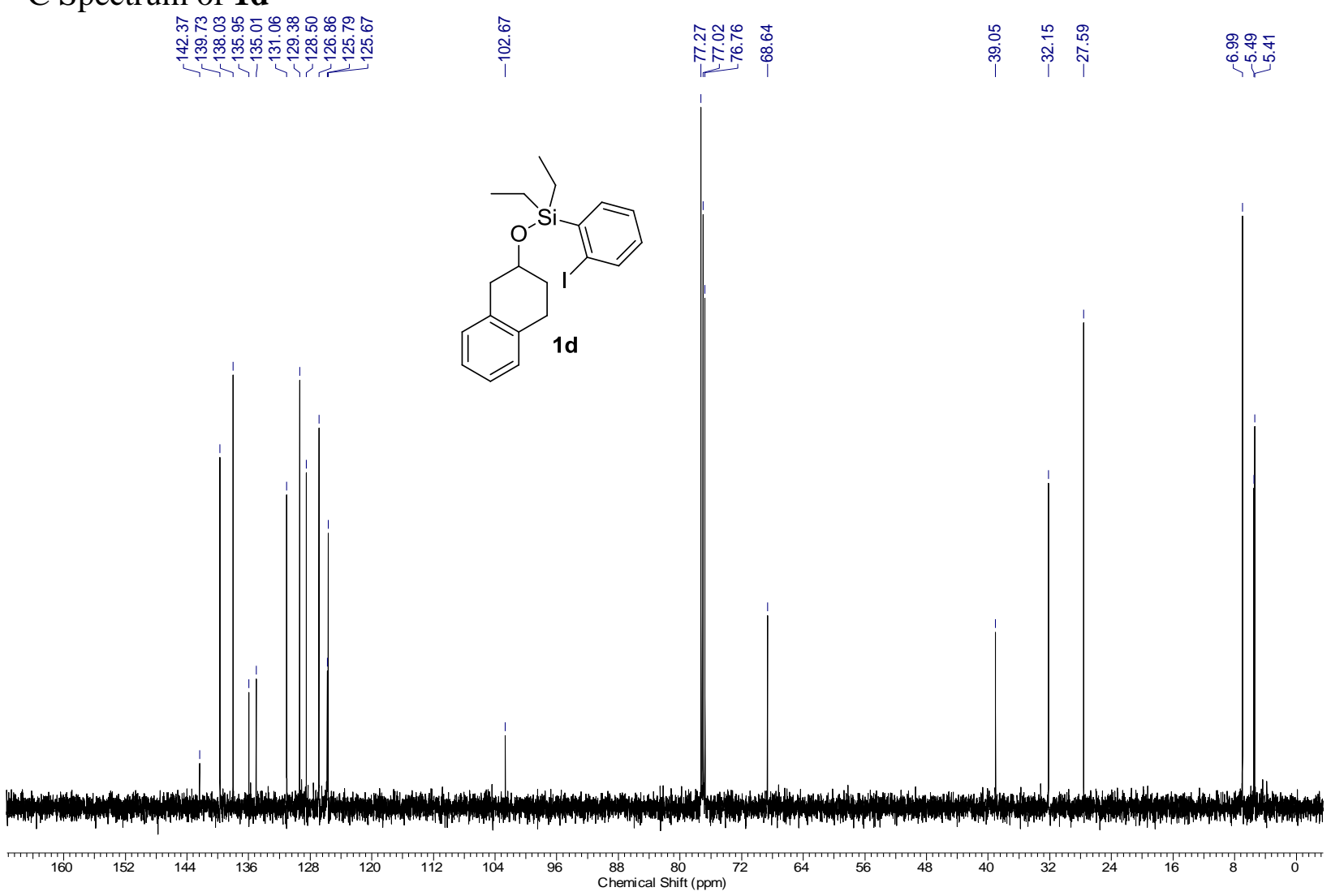


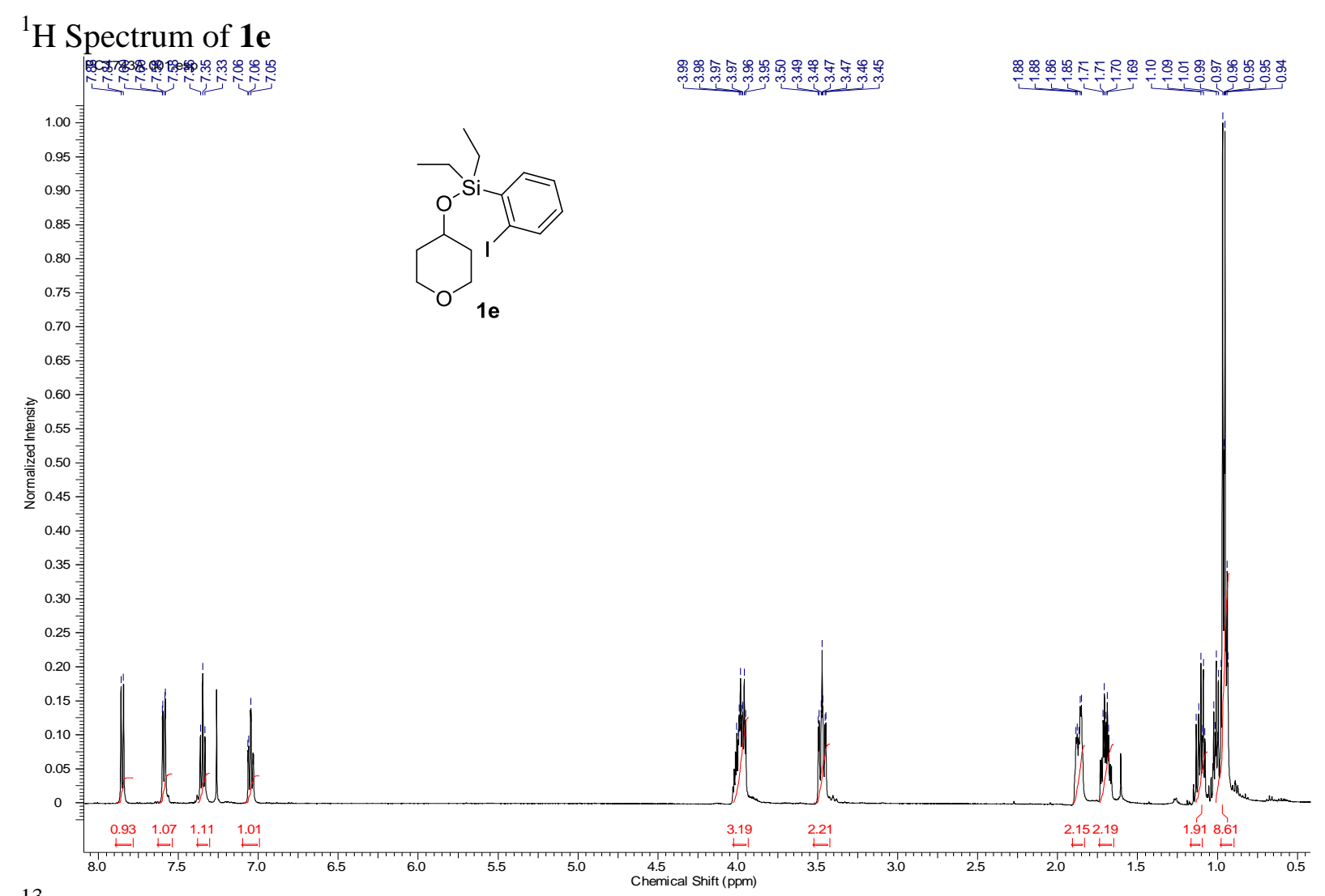

\section{${ }^{13} \mathrm{C}$ Spectrum of $\mathbf{1 e}$}

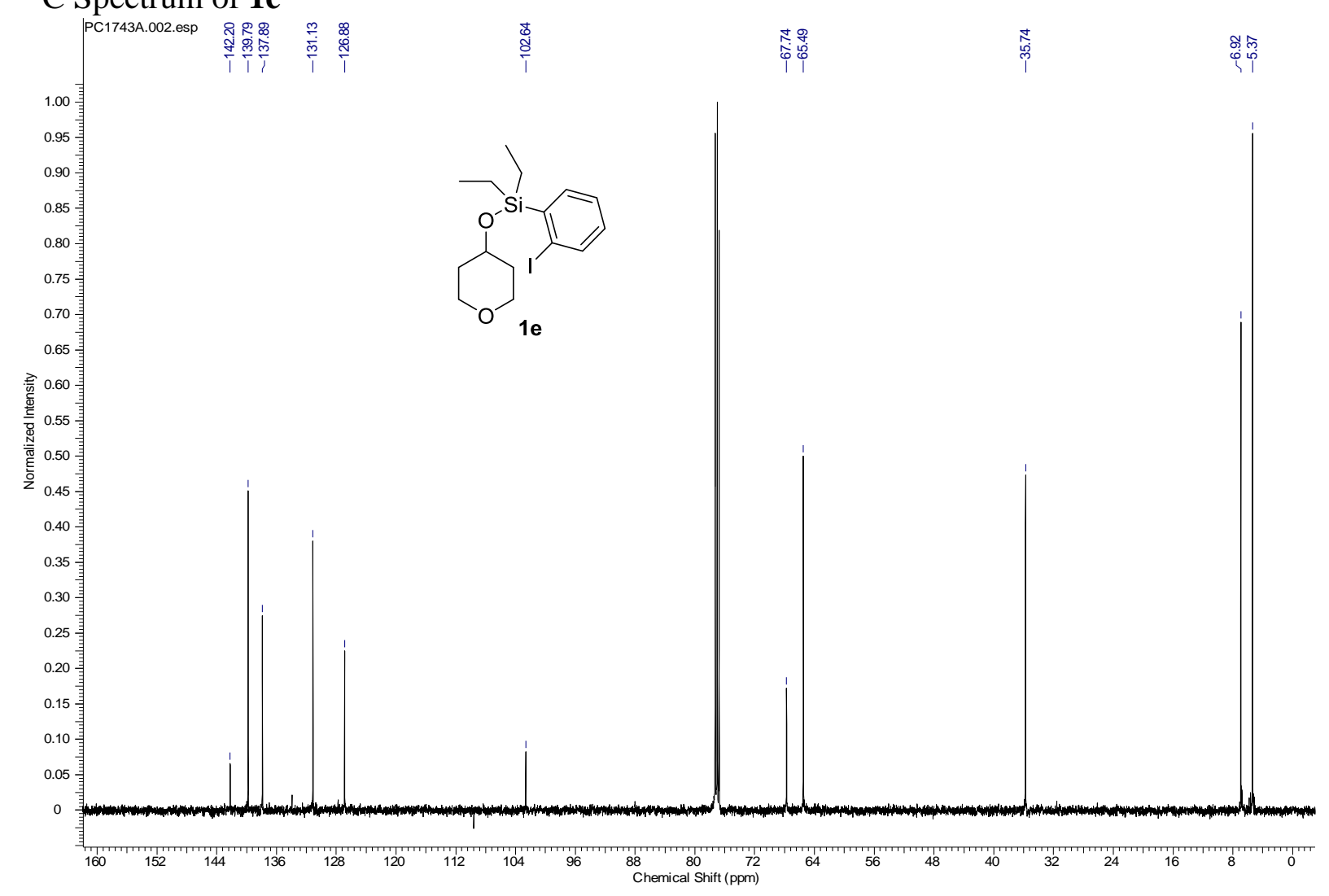




\section{${ }^{1} \mathrm{H}$ Spectrum of $\mathbf{1 f}$}

管

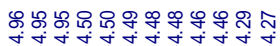

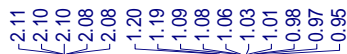<smiles>CC[Si](CC)(OC1CCOc2ccccc21)c1ccccc1I</smiles>

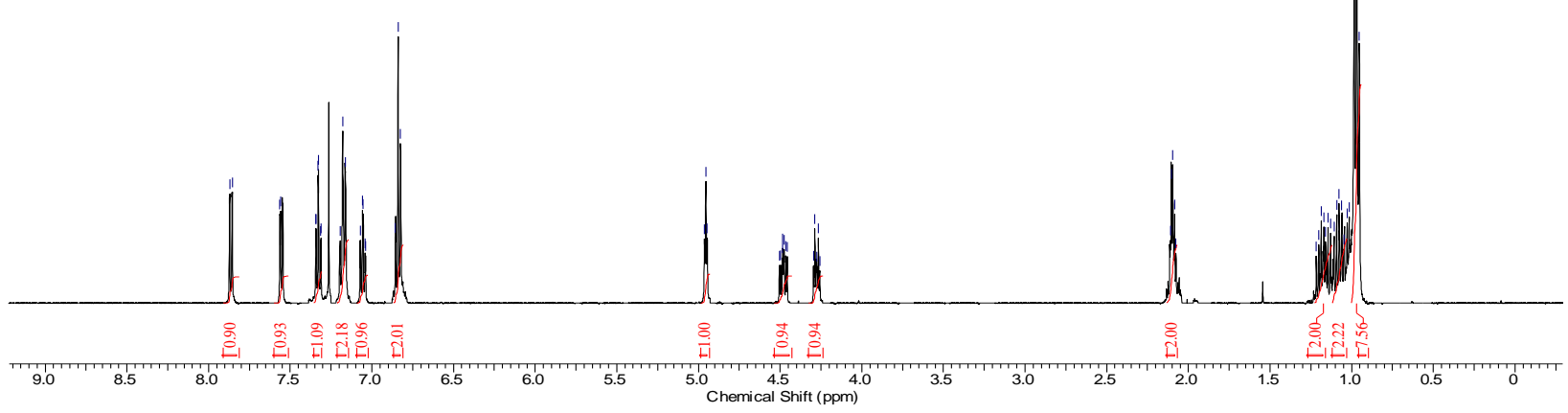

${ }^{13} \mathrm{C}$ Spectrum of $\mathbf{1 f}$
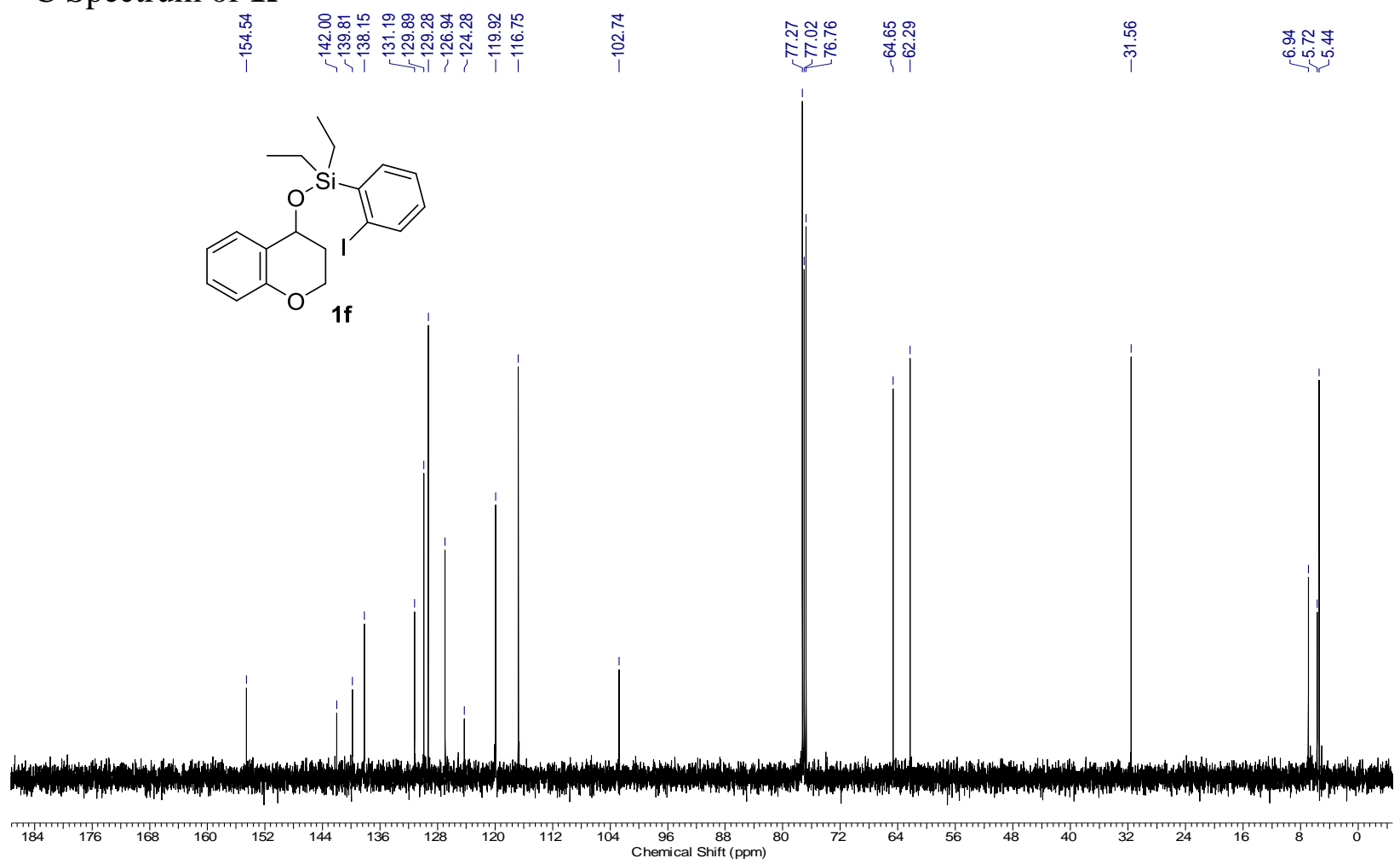


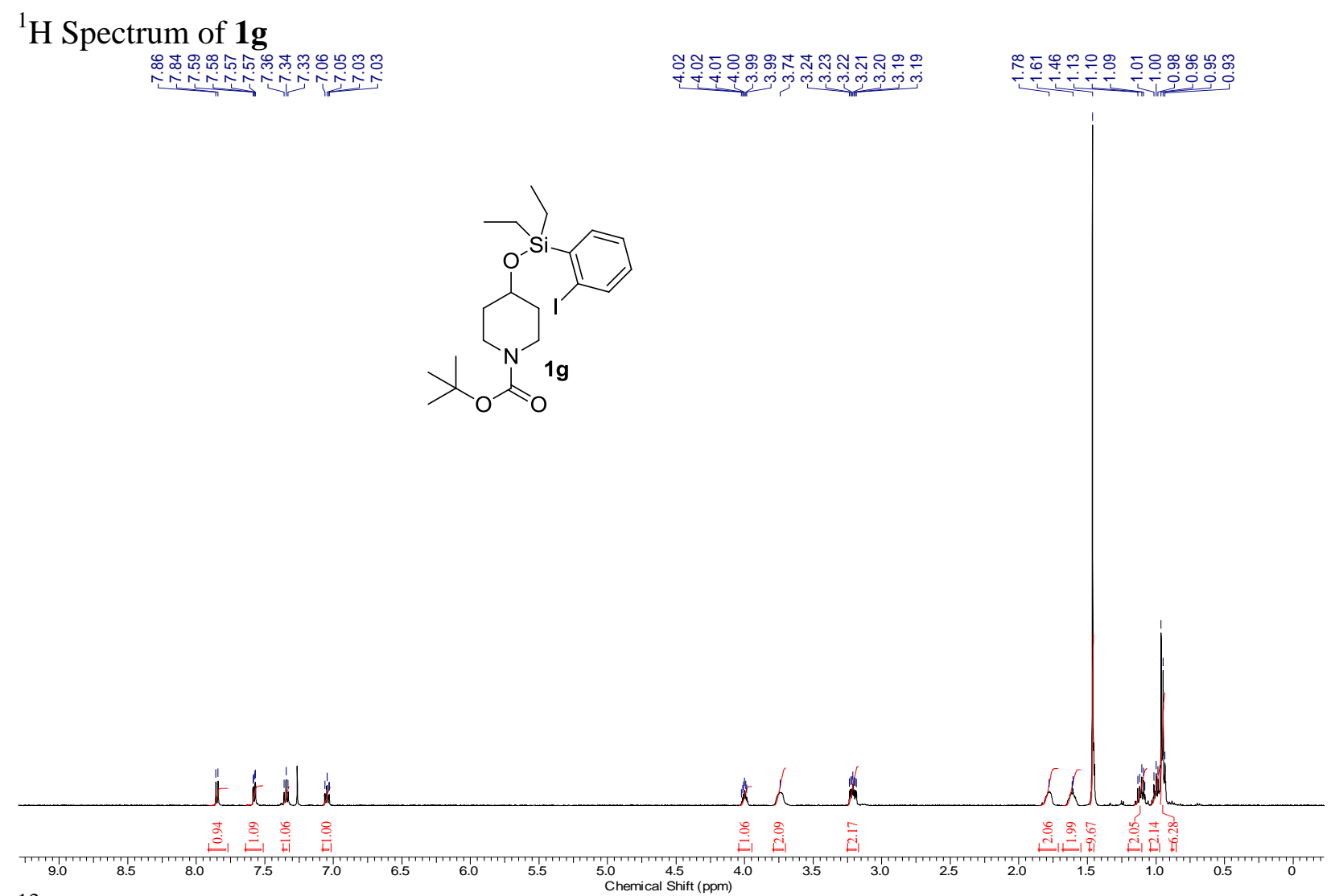

${ }^{13} \mathrm{C}$ Spectrum of $\mathbf{1 g}$

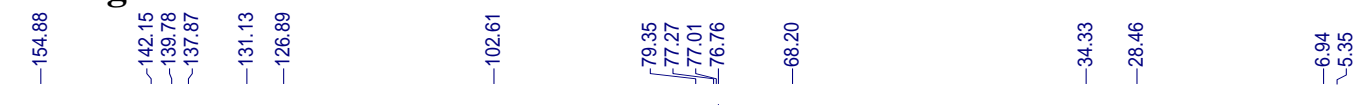
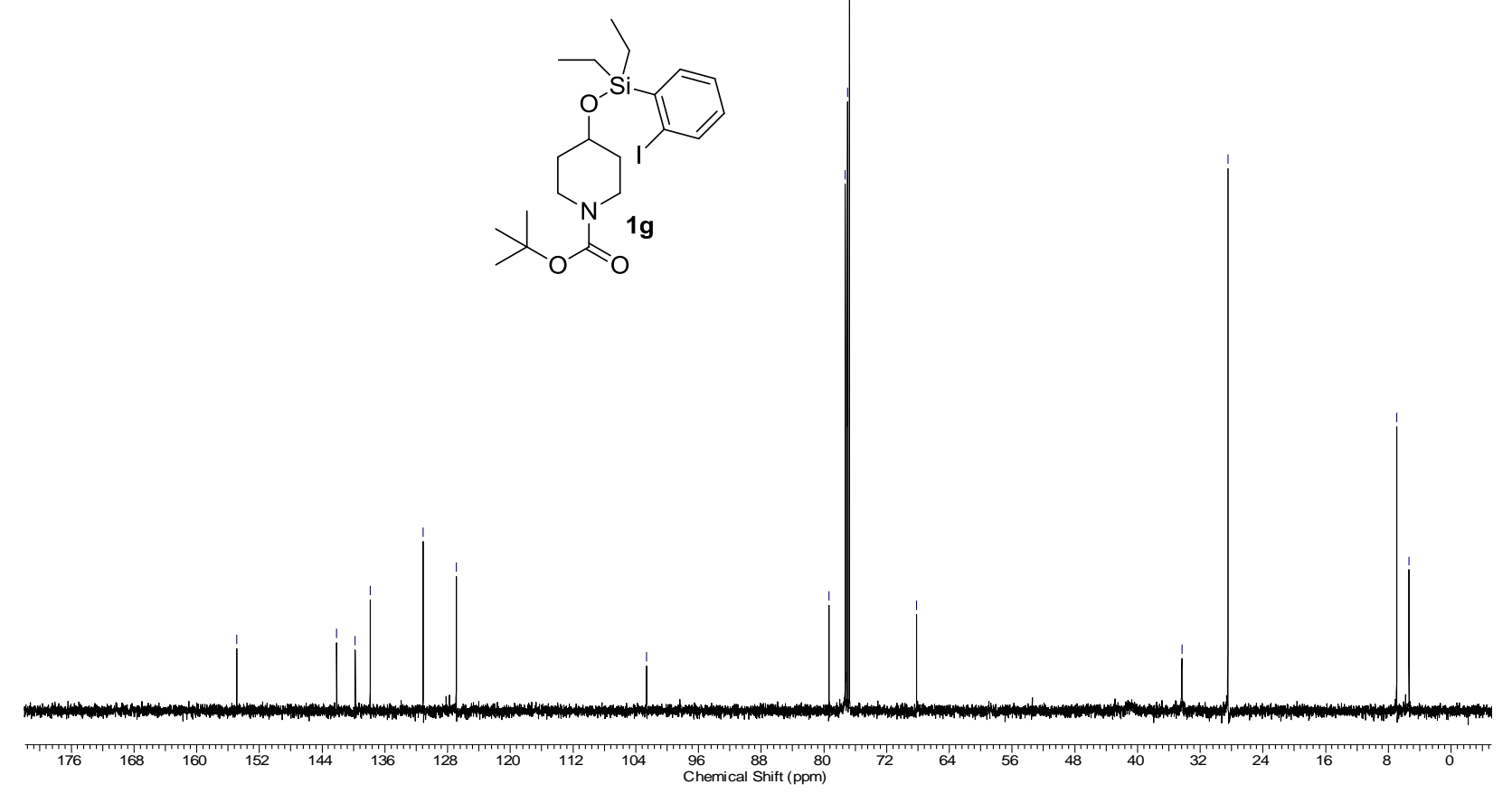


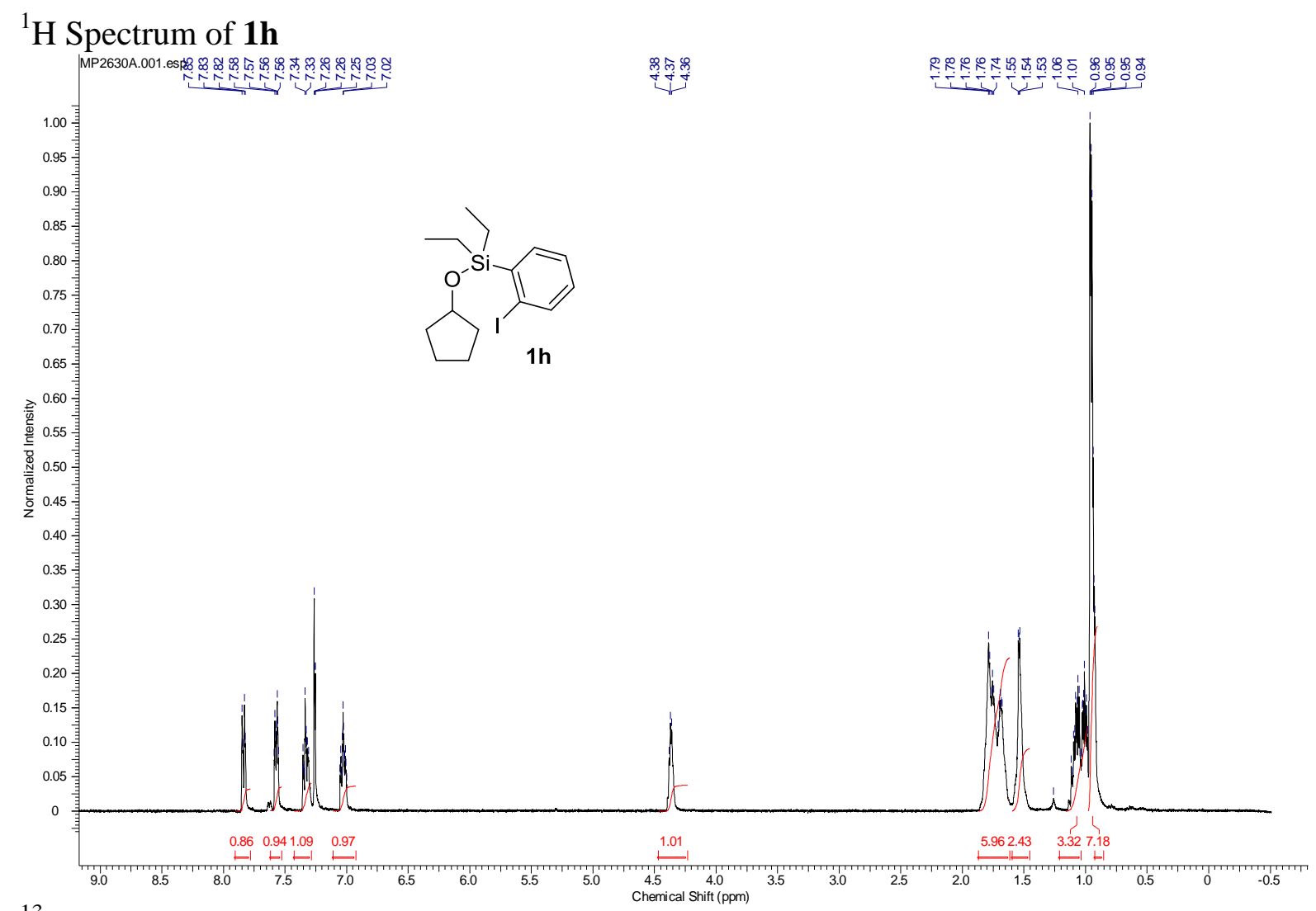

${ }^{13} \mathrm{C}$ Spectrum of $\mathbf{1 h}$

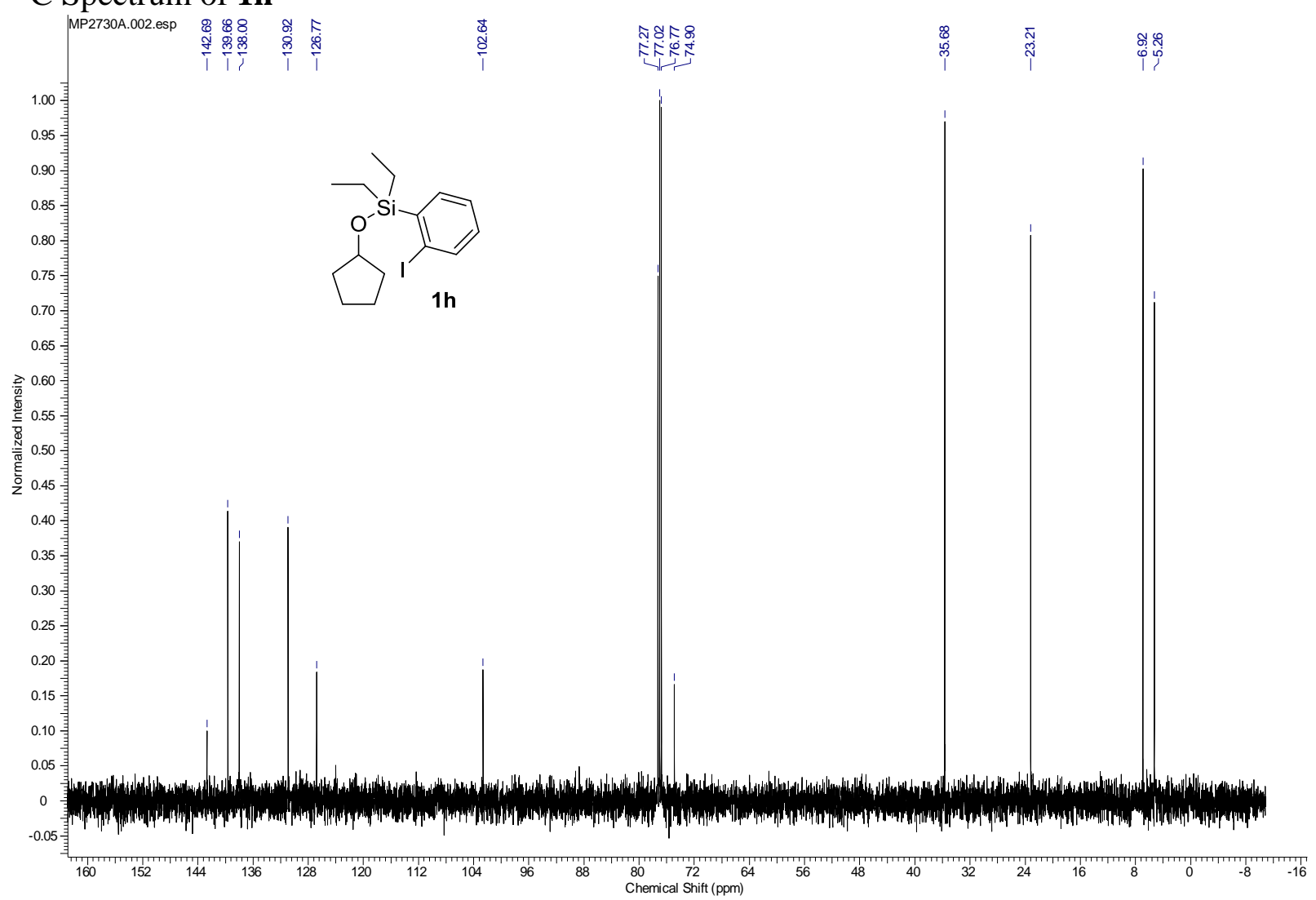




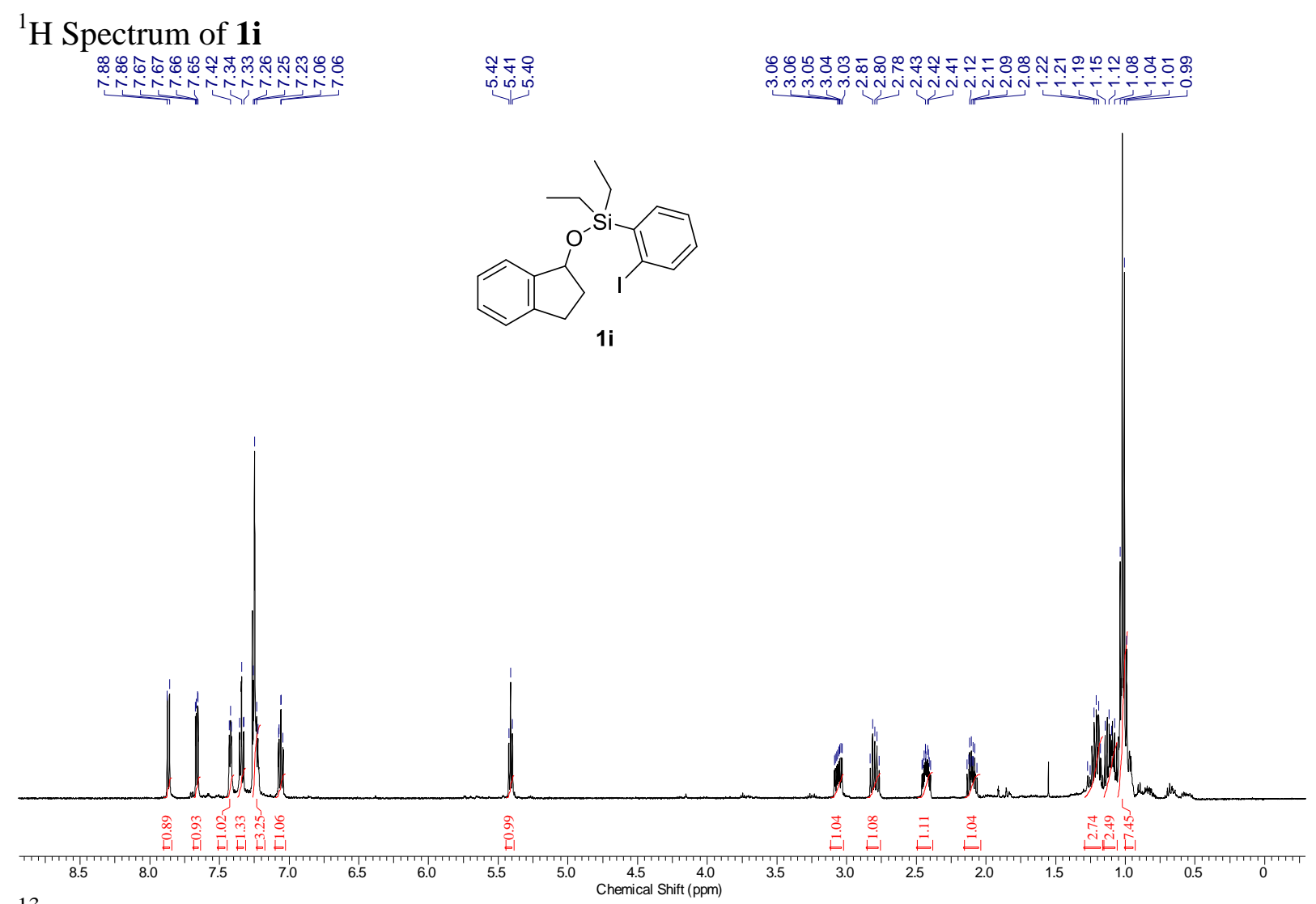

${ }^{13} \mathrm{C}$ Spectrum of $\mathbf{1 i}$
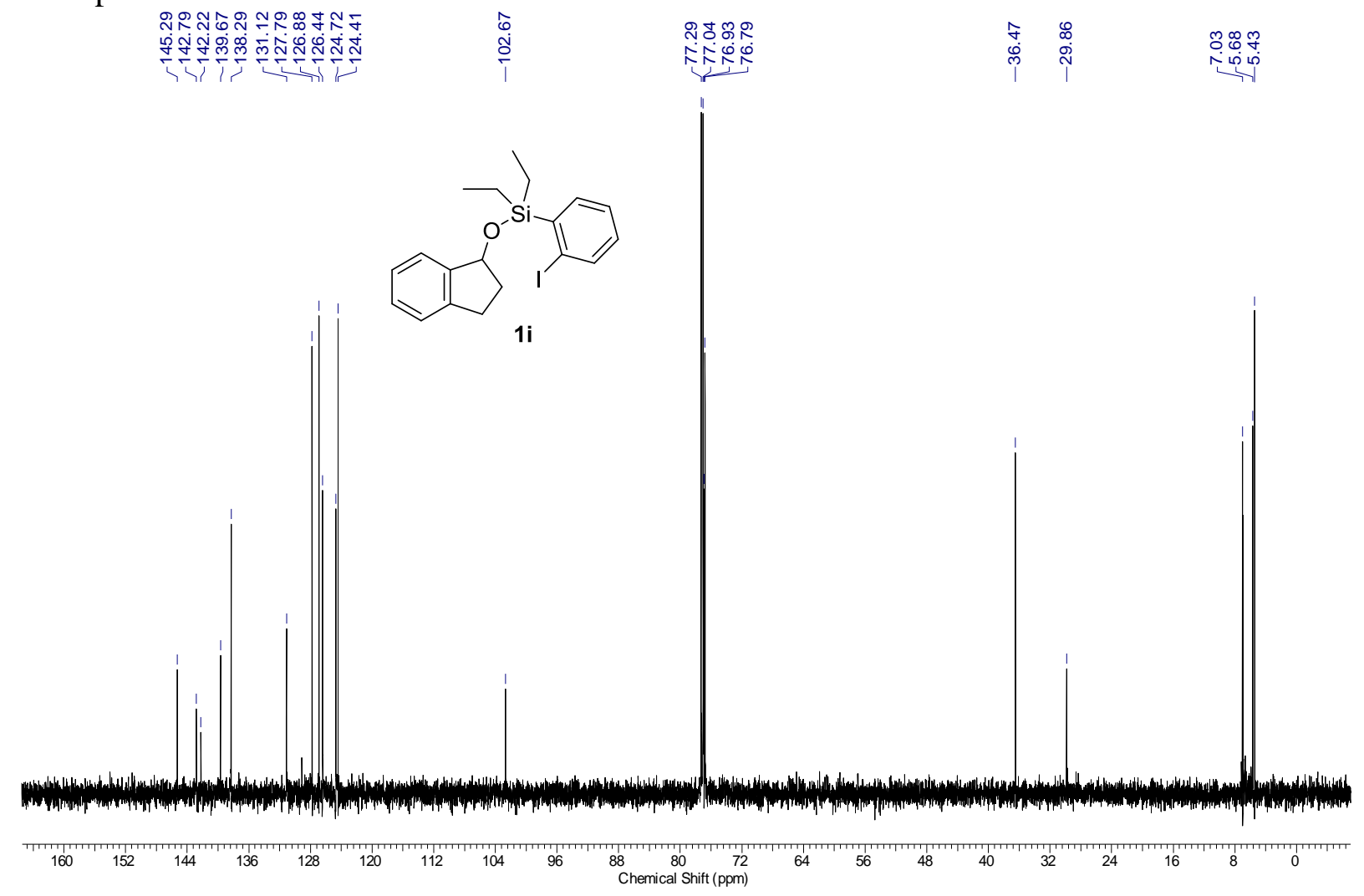


\section{${ }^{1} \mathrm{H}$ Spectrum of $\mathbf{1 j}$}

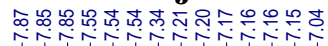

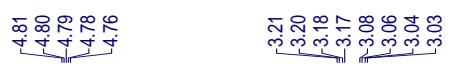

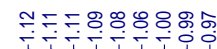

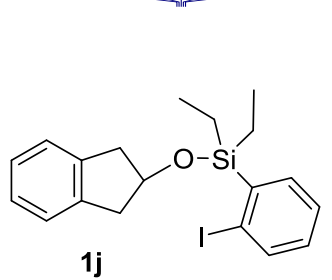

1j
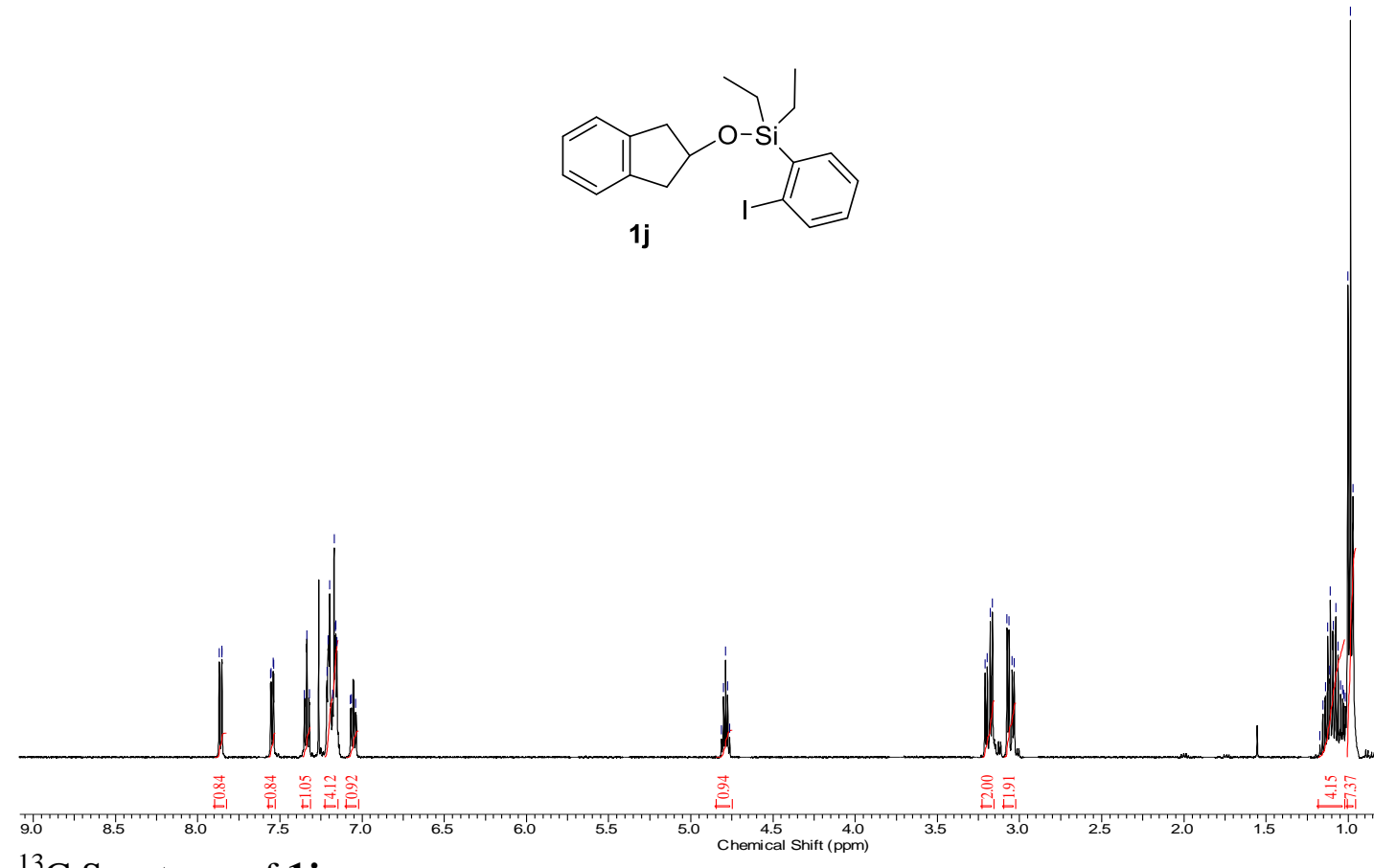

${ }^{13} \mathrm{C}$ Spectrum of $\mathbf{1 j}$

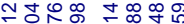

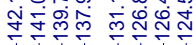

$\begin{array}{lll}1 & 0 \\ 0 & 0 \\ 0 & 0\end{array}$

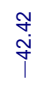

하욤

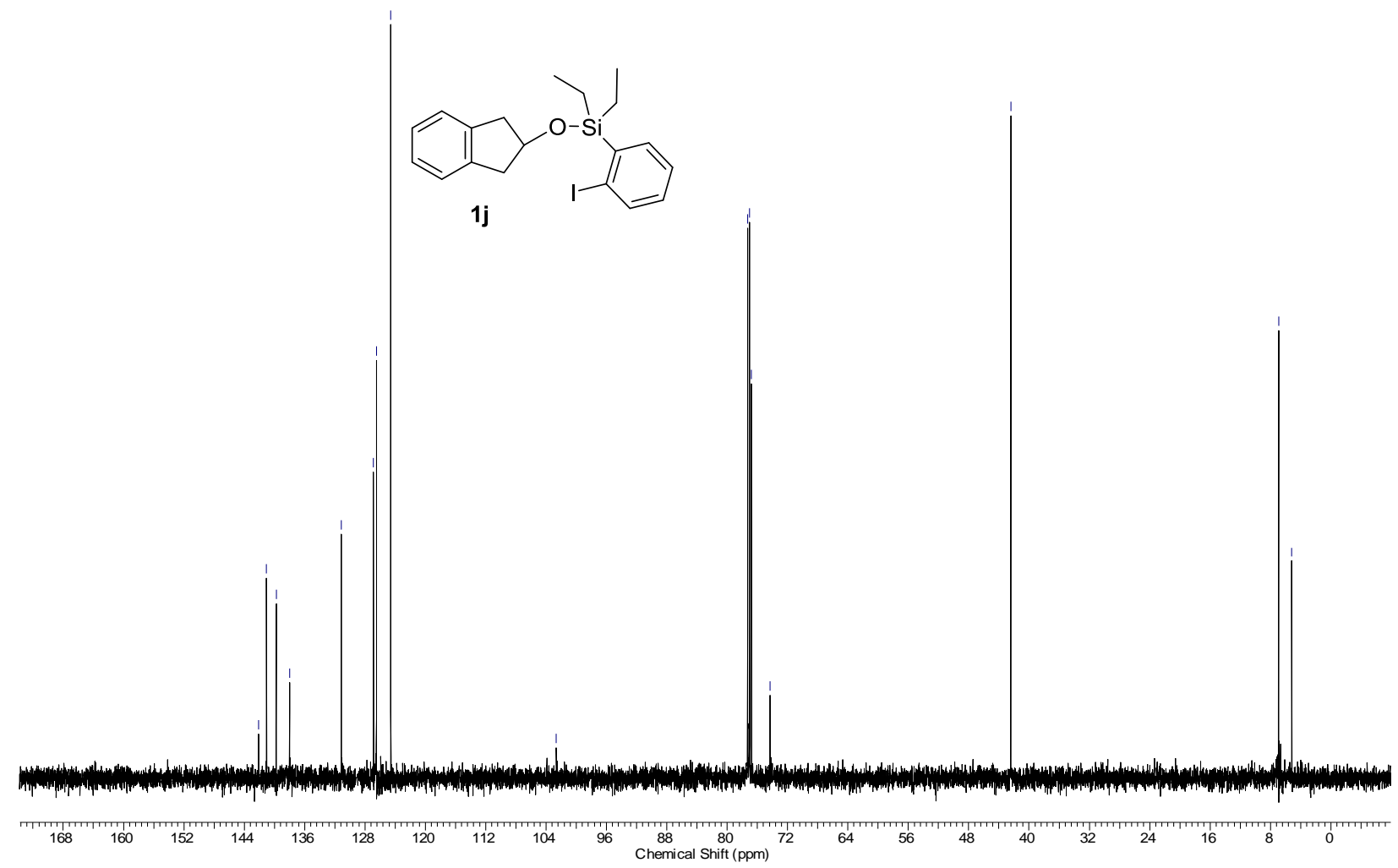


${ }^{1} \mathrm{H}$ Spectrum of $\mathbf{1 k}$

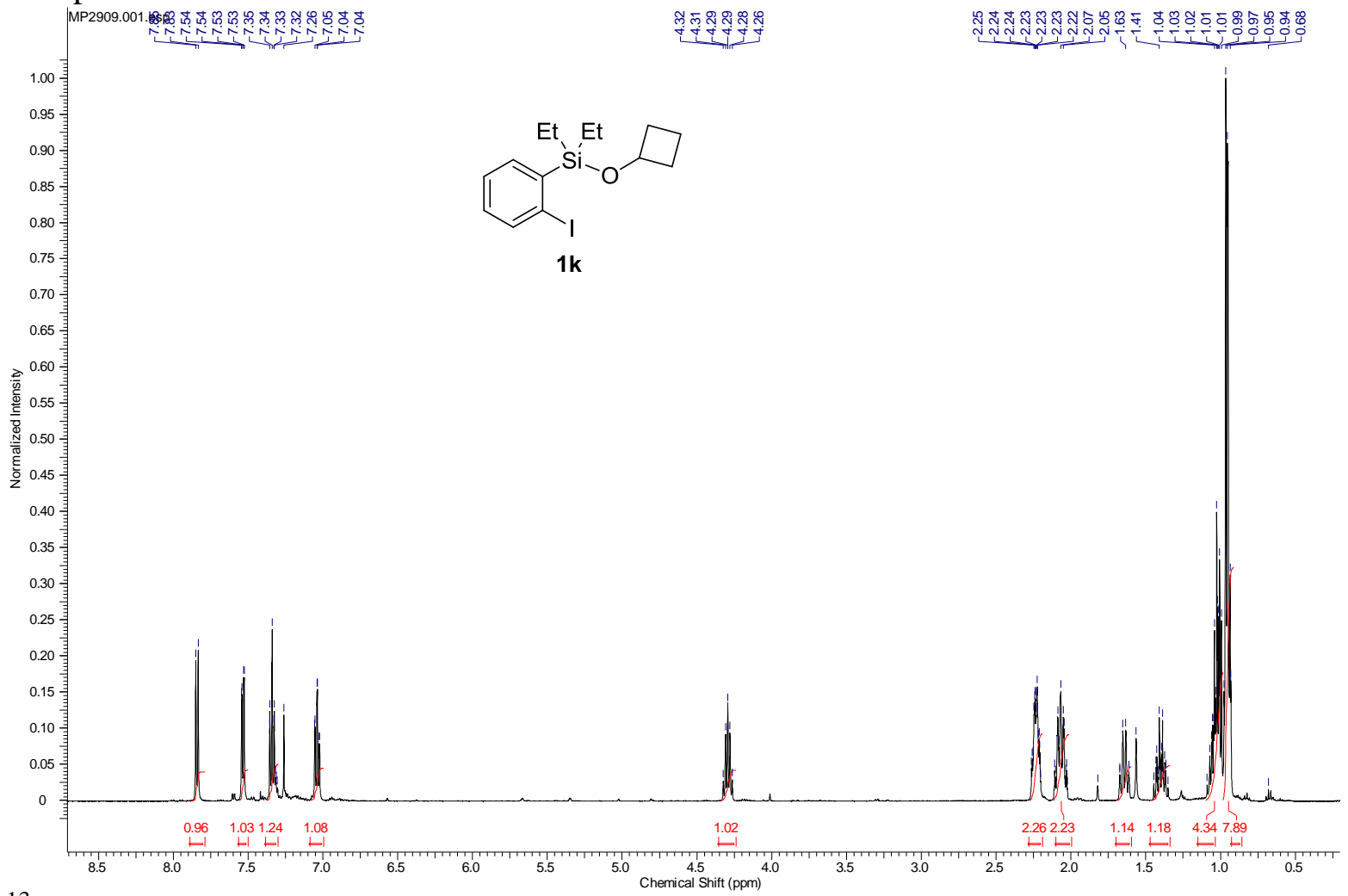

${ }^{13} \mathrm{C}$ Spectrum of $\mathbf{1 k}$

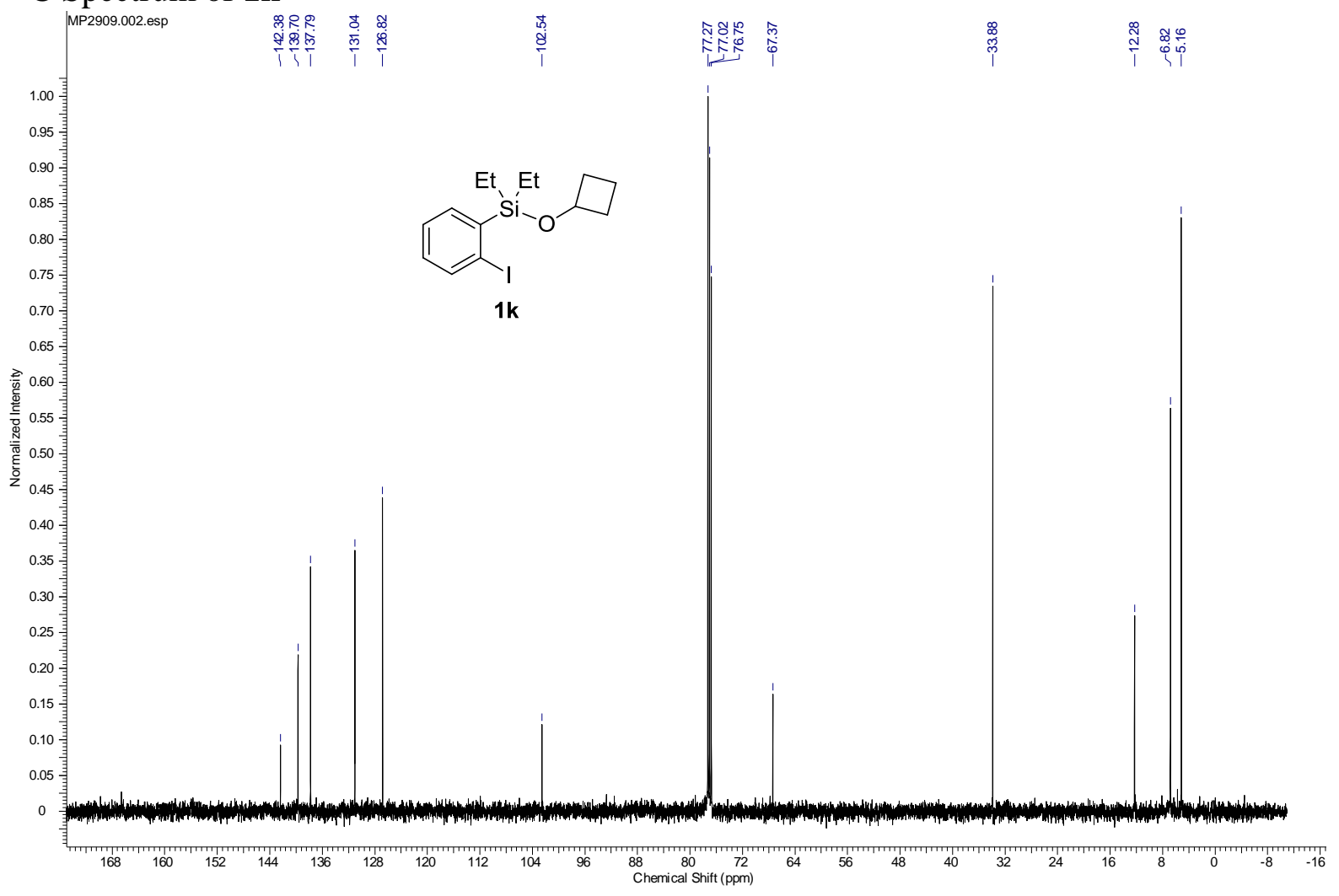




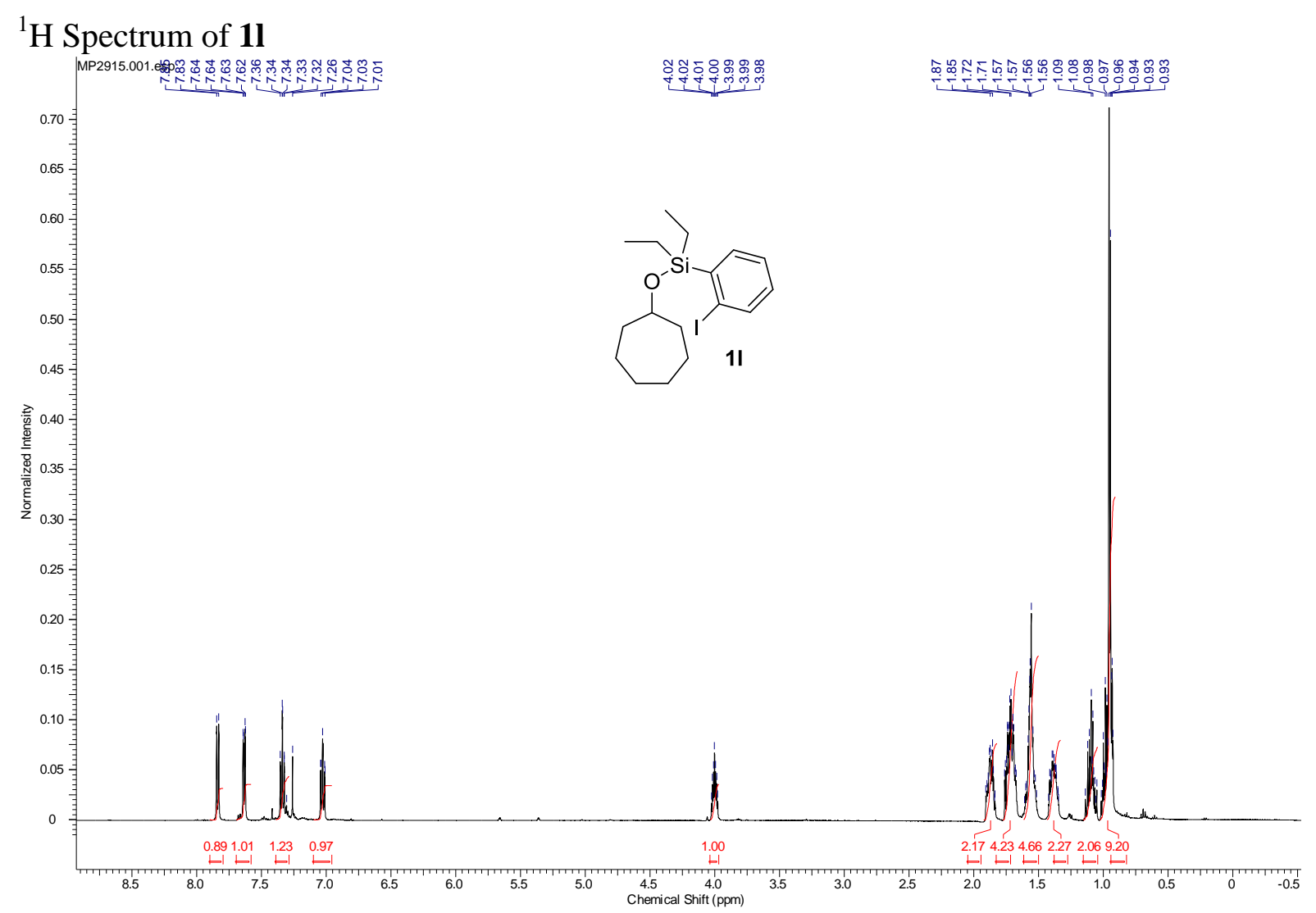

${ }^{13} \mathrm{C}$ Spectrum of $\mathbf{1 1}$

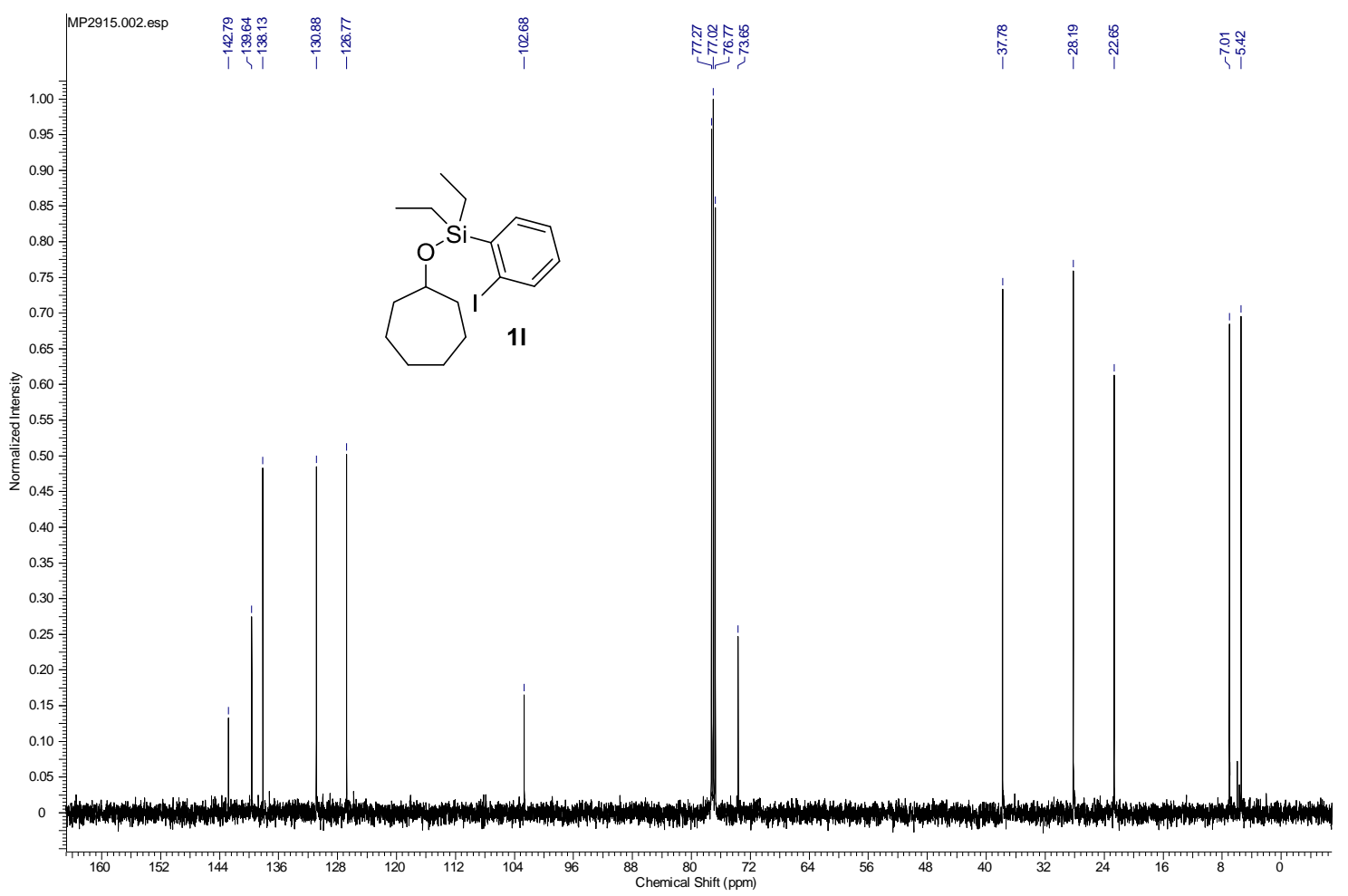




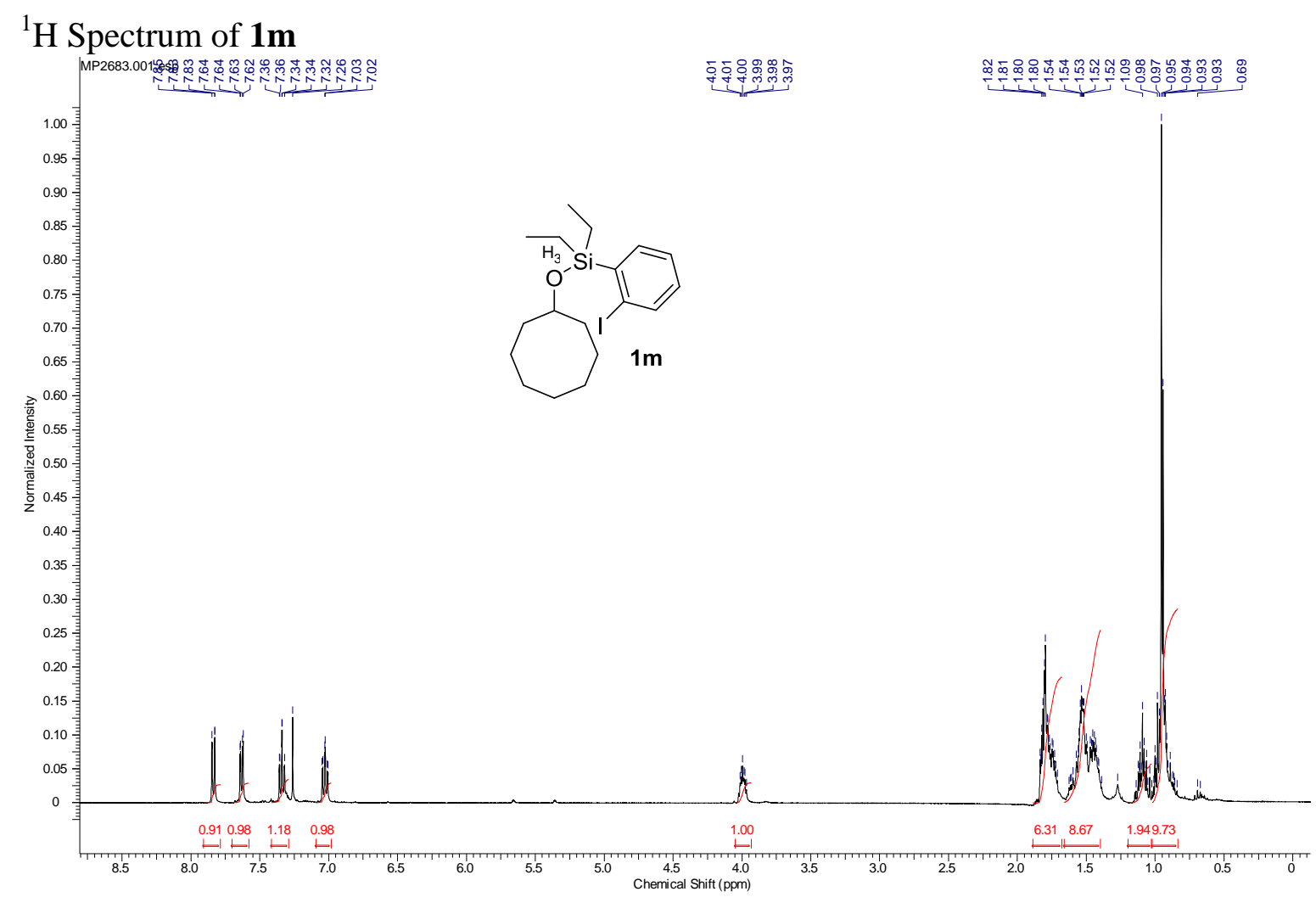

${ }^{13} \mathrm{C}$ Spectrum of $\mathbf{1 m}$

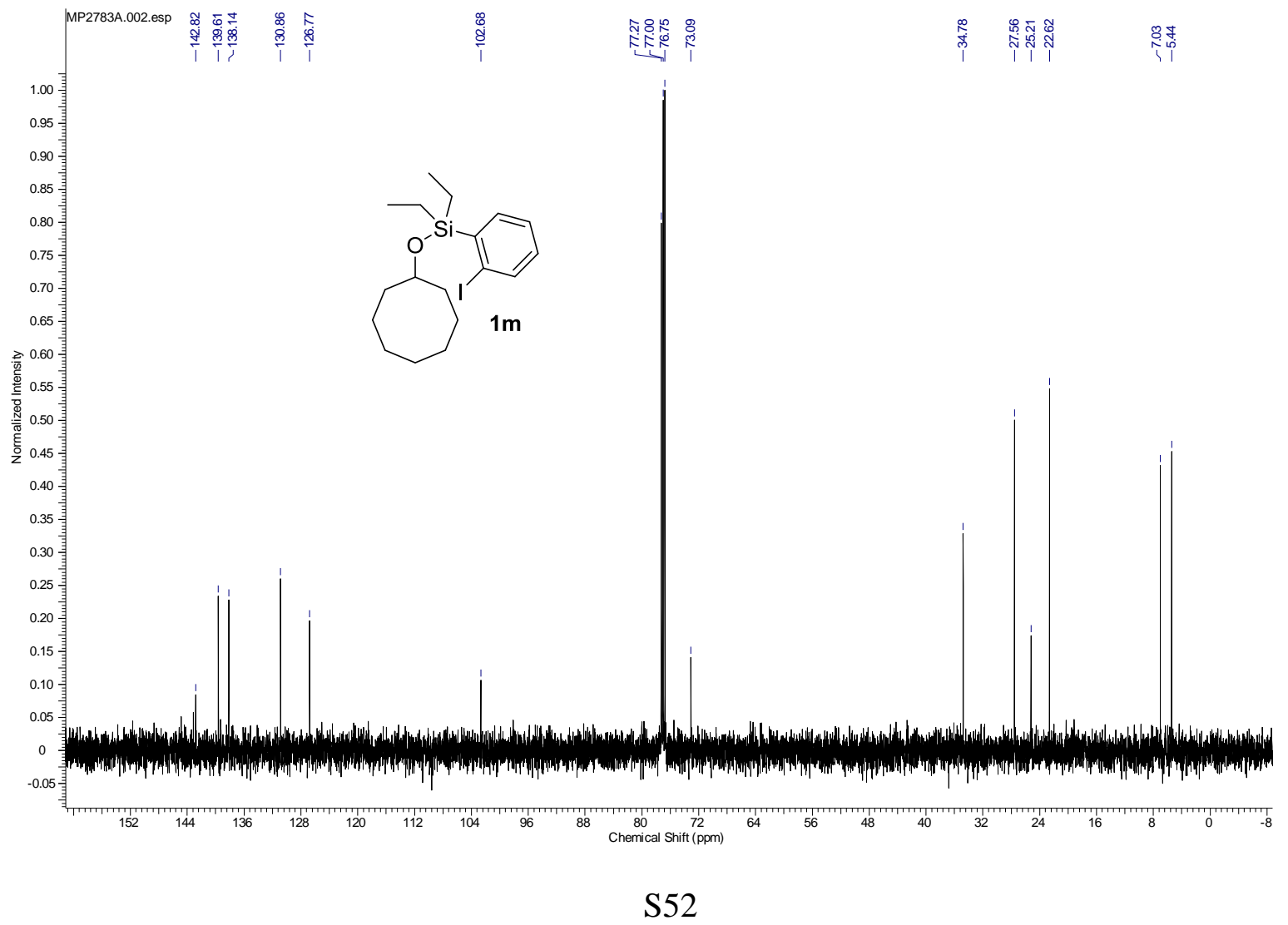



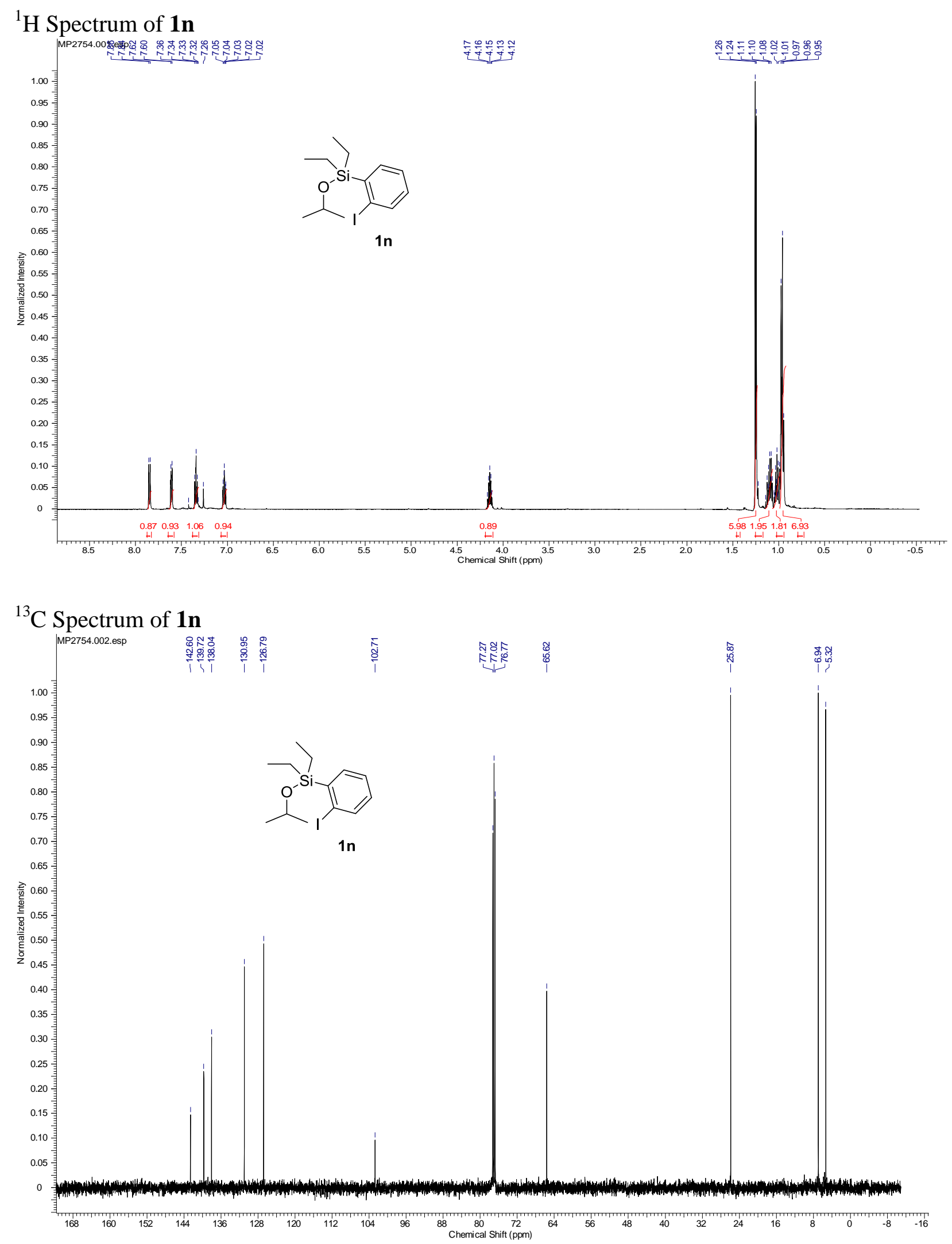


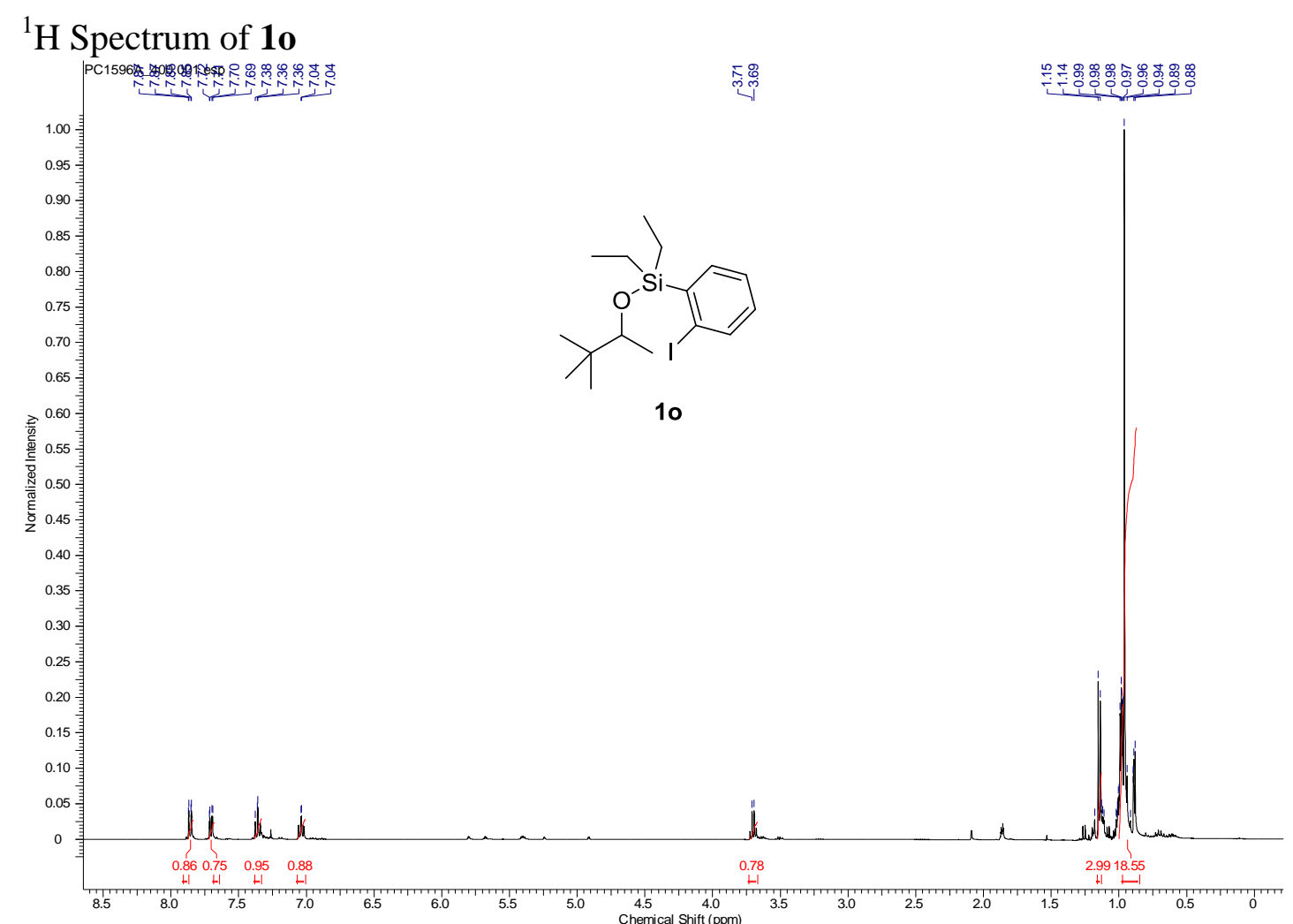

${ }^{13} \mathrm{C}$ Spectrum of 10

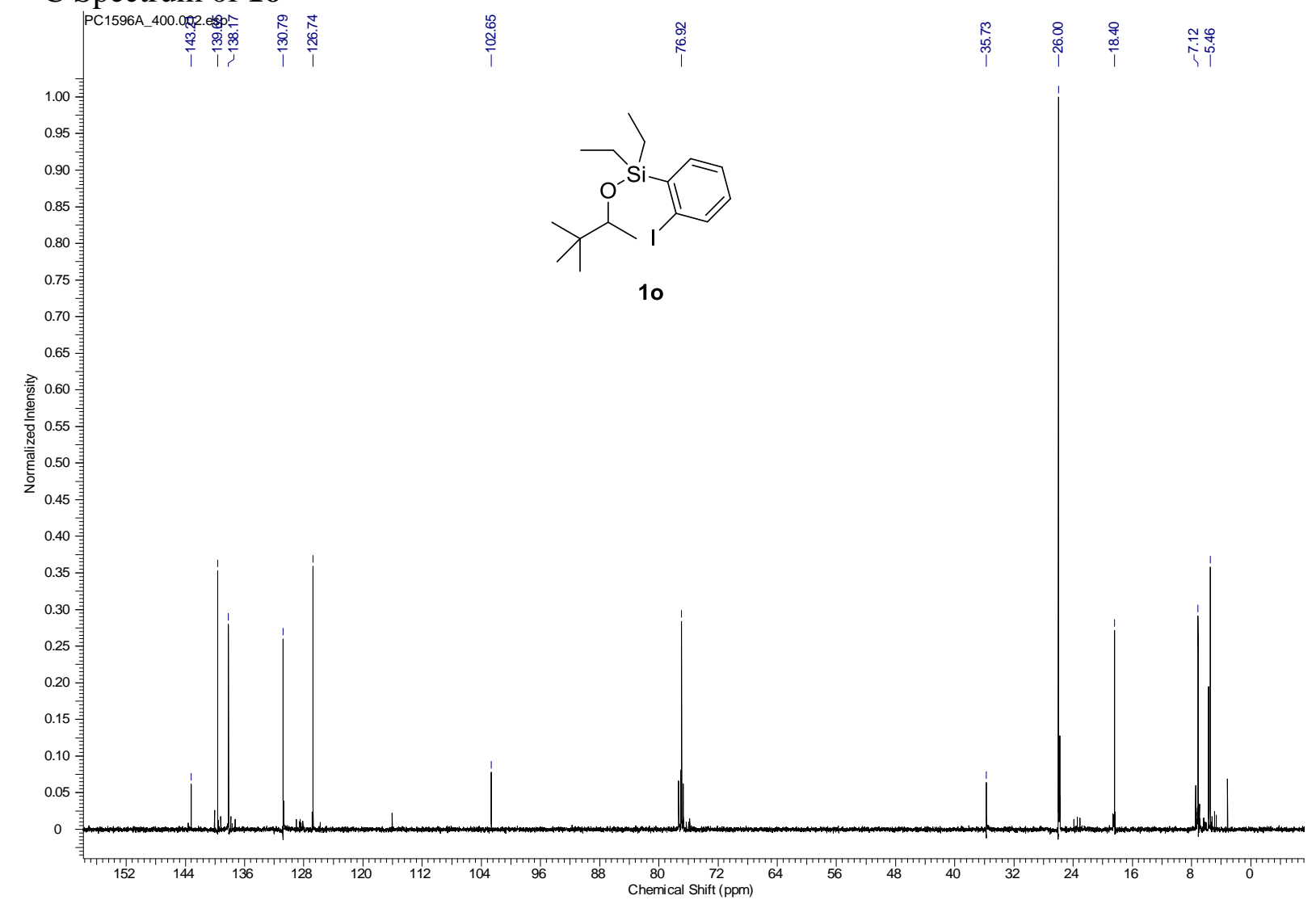




\section{${ }^{1} \mathrm{H}$ Spectrum of $\mathbf{1 p}$}
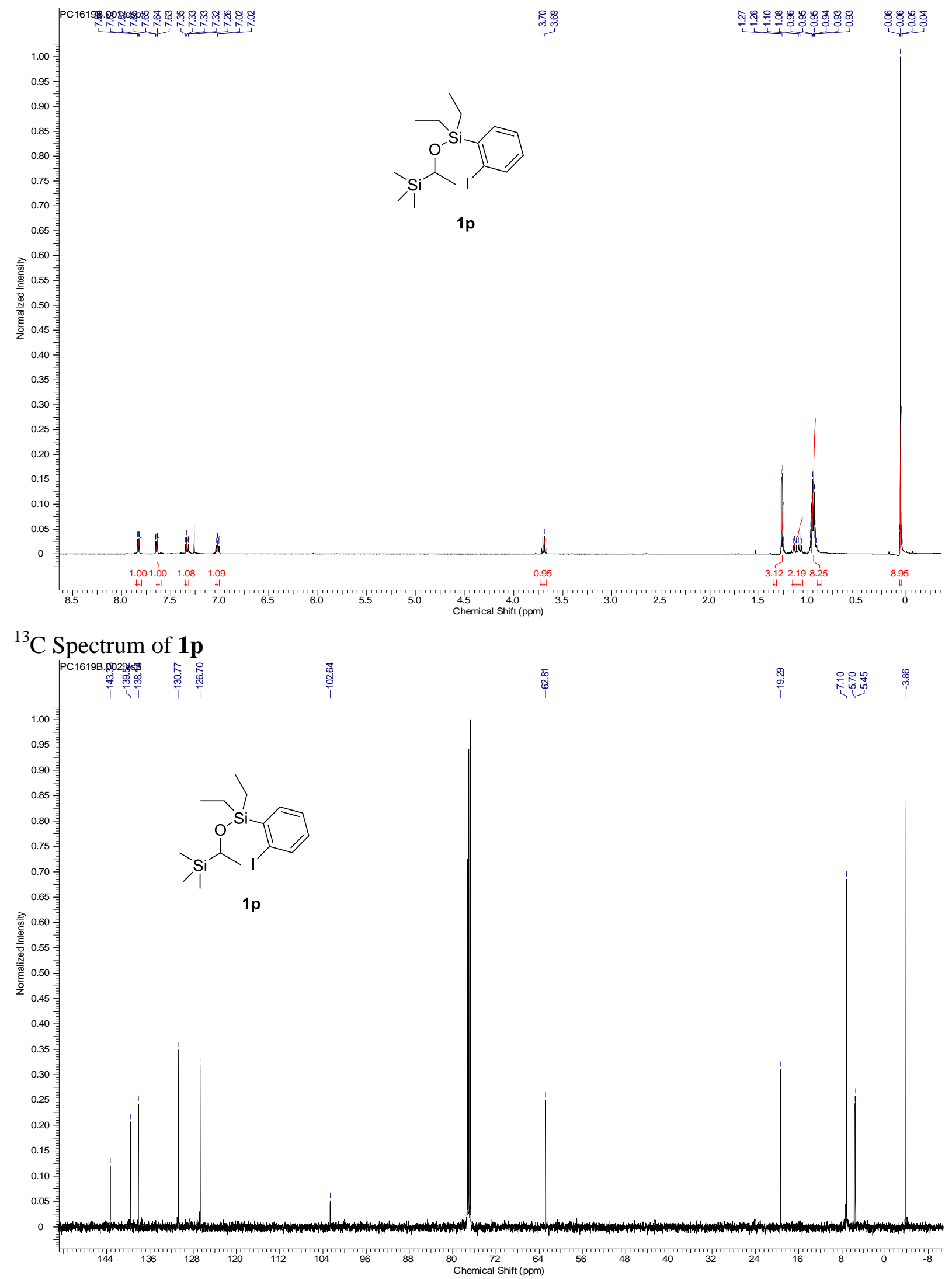


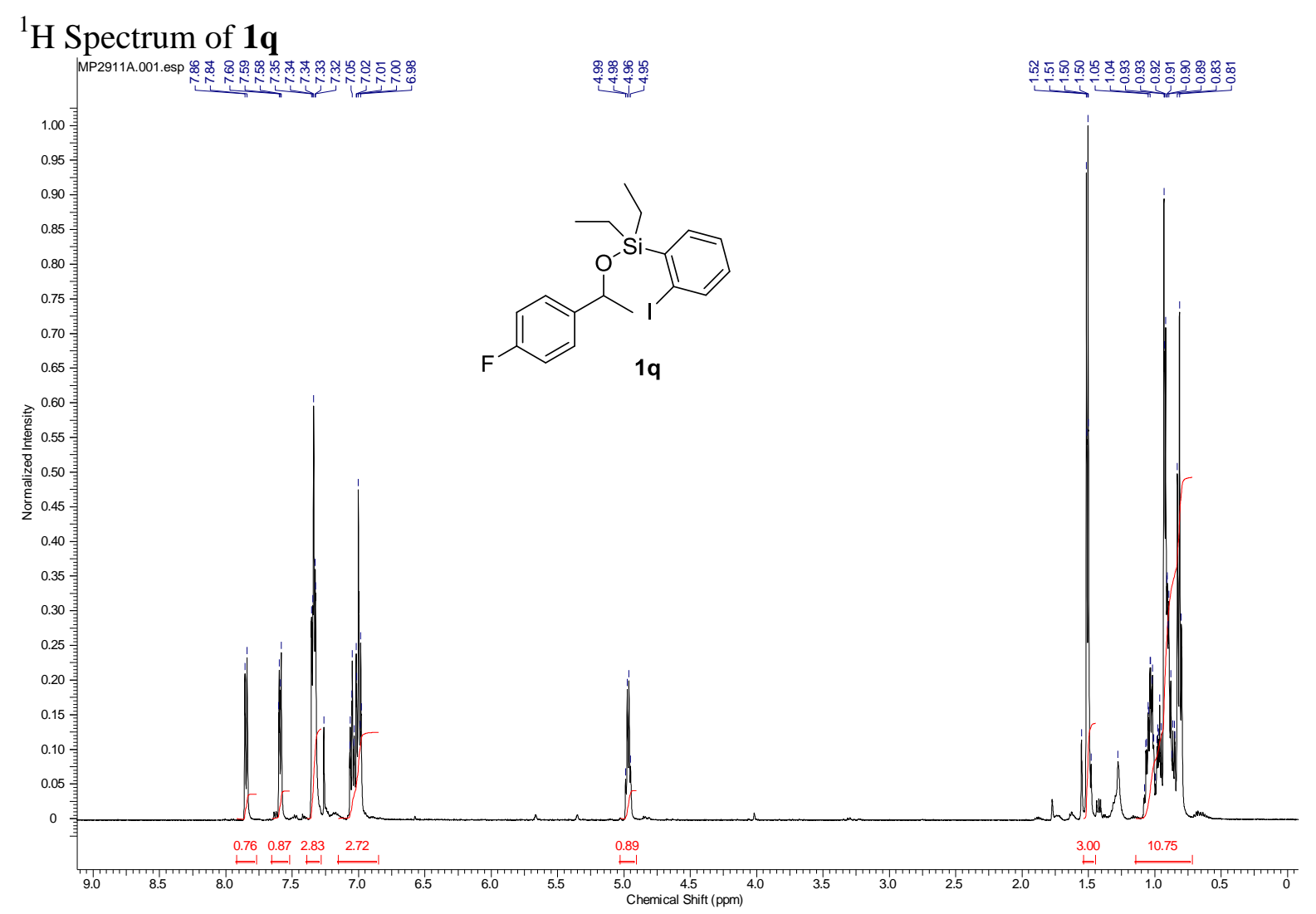

${ }^{13} \mathrm{C}$ Spectrum of $\mathbf{1 q}$

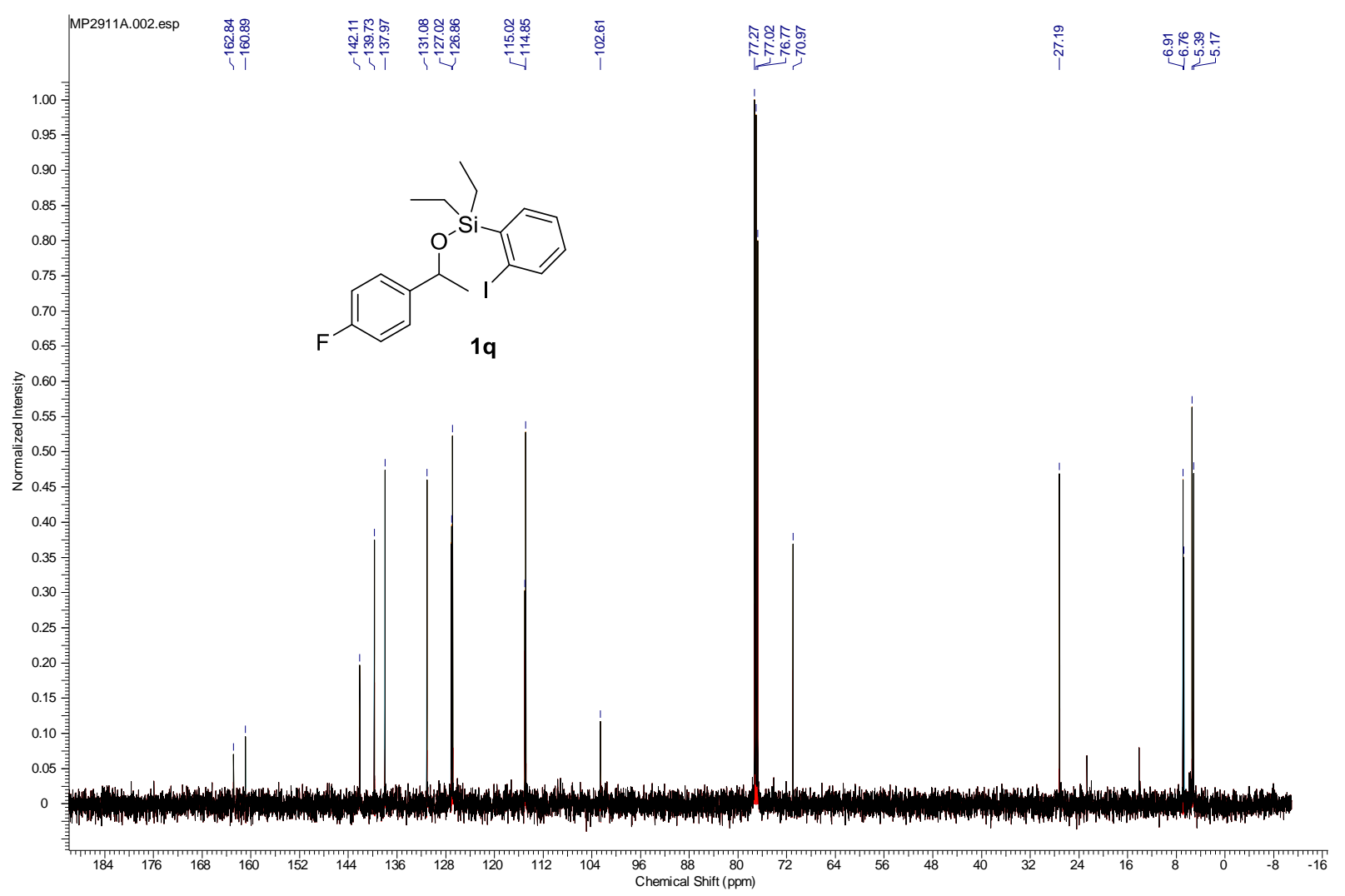


${ }^{1} \mathrm{H}$ Spectrum of $\mathbf{1 r}$

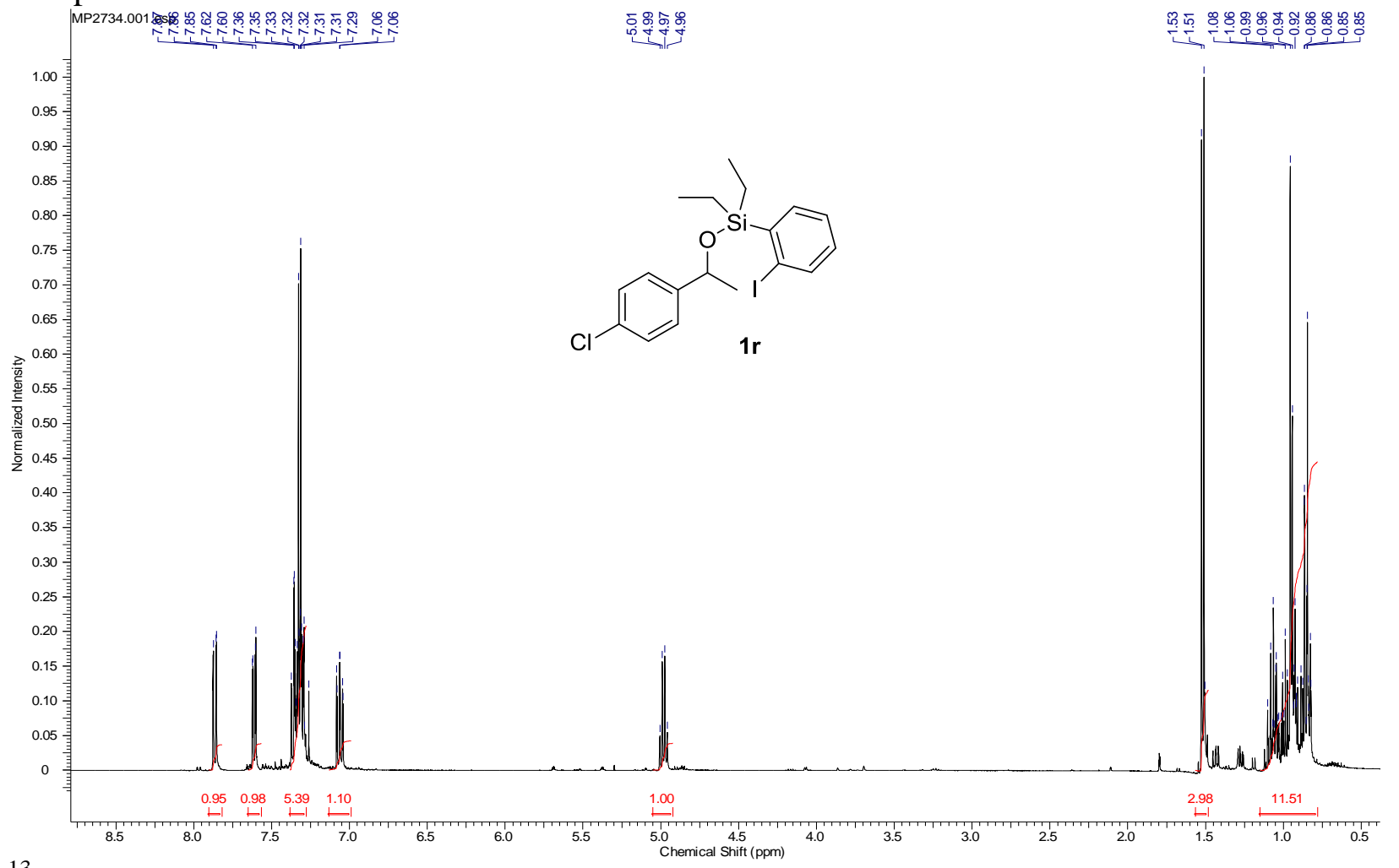

${ }^{13} \mathrm{C}$ Spectrum of $\mathbf{1 r}$

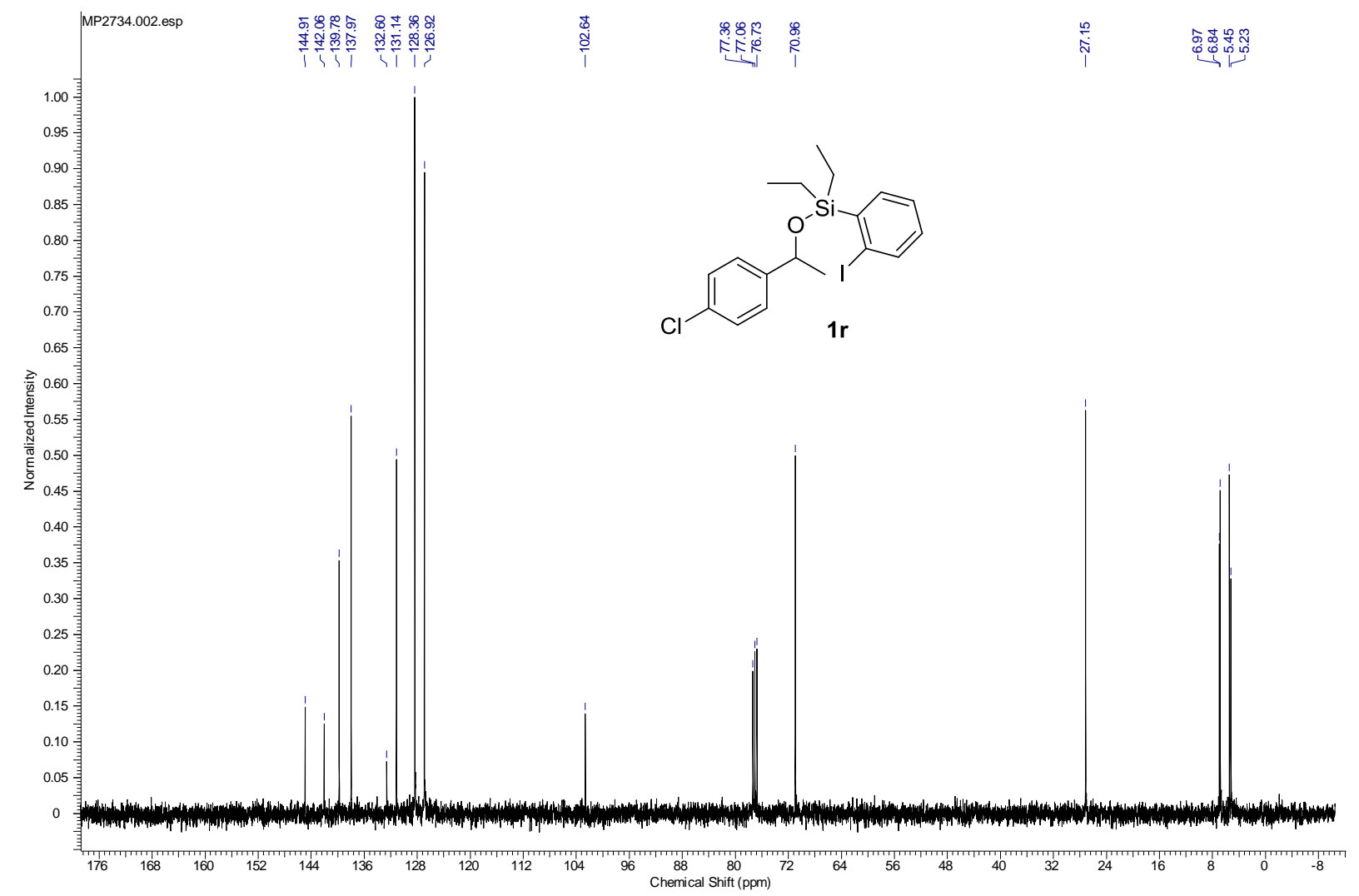




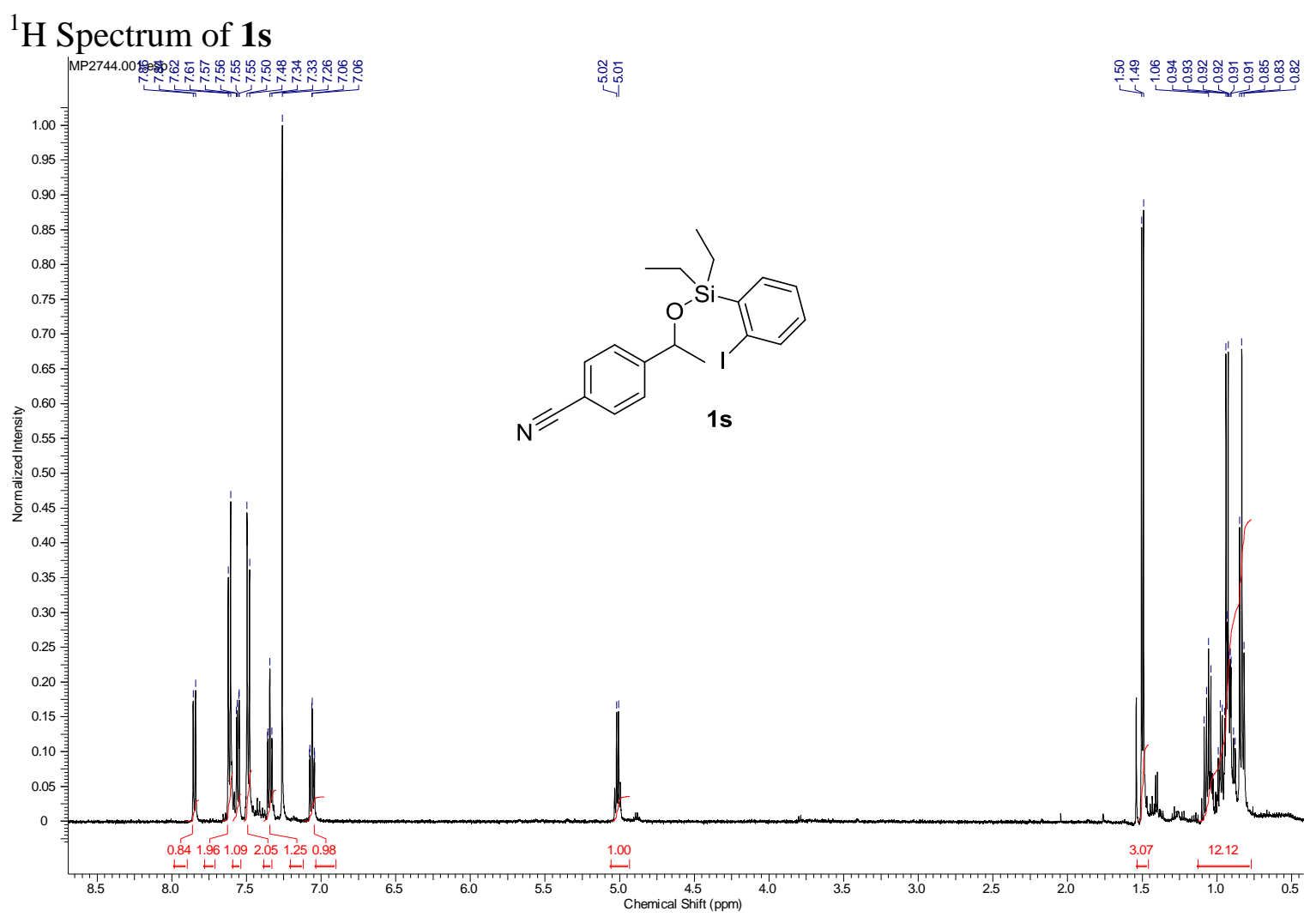

\section{${ }^{13} \mathrm{C}$ Spectrum of $1 \mathrm{~s}$}

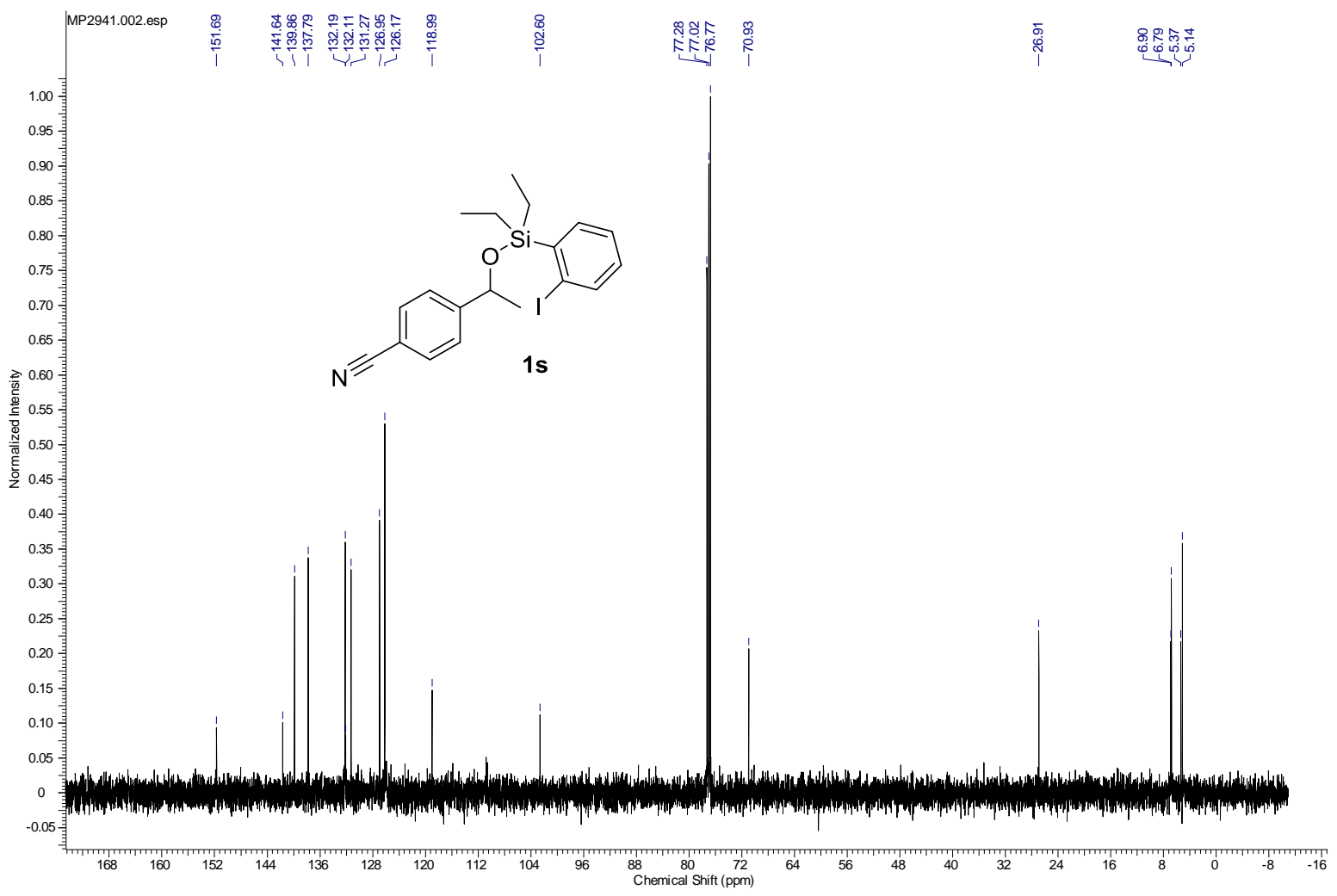




\section{${ }^{1} \mathrm{H}$ Spectrum of $\mathbf{1 t}$}

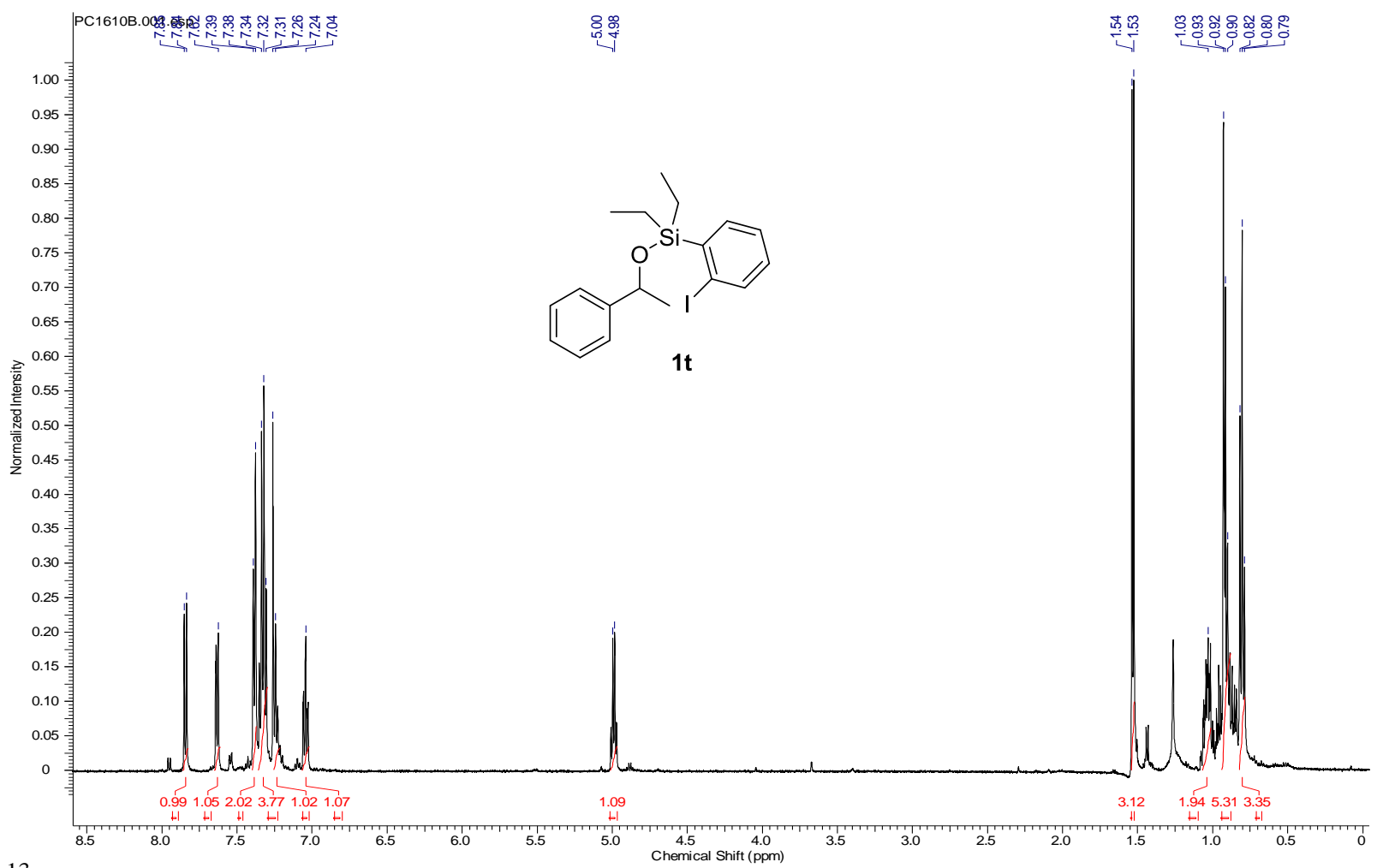

\section{${ }^{13} \mathrm{C}$ Spectrum of $\mathbf{1 t}$}

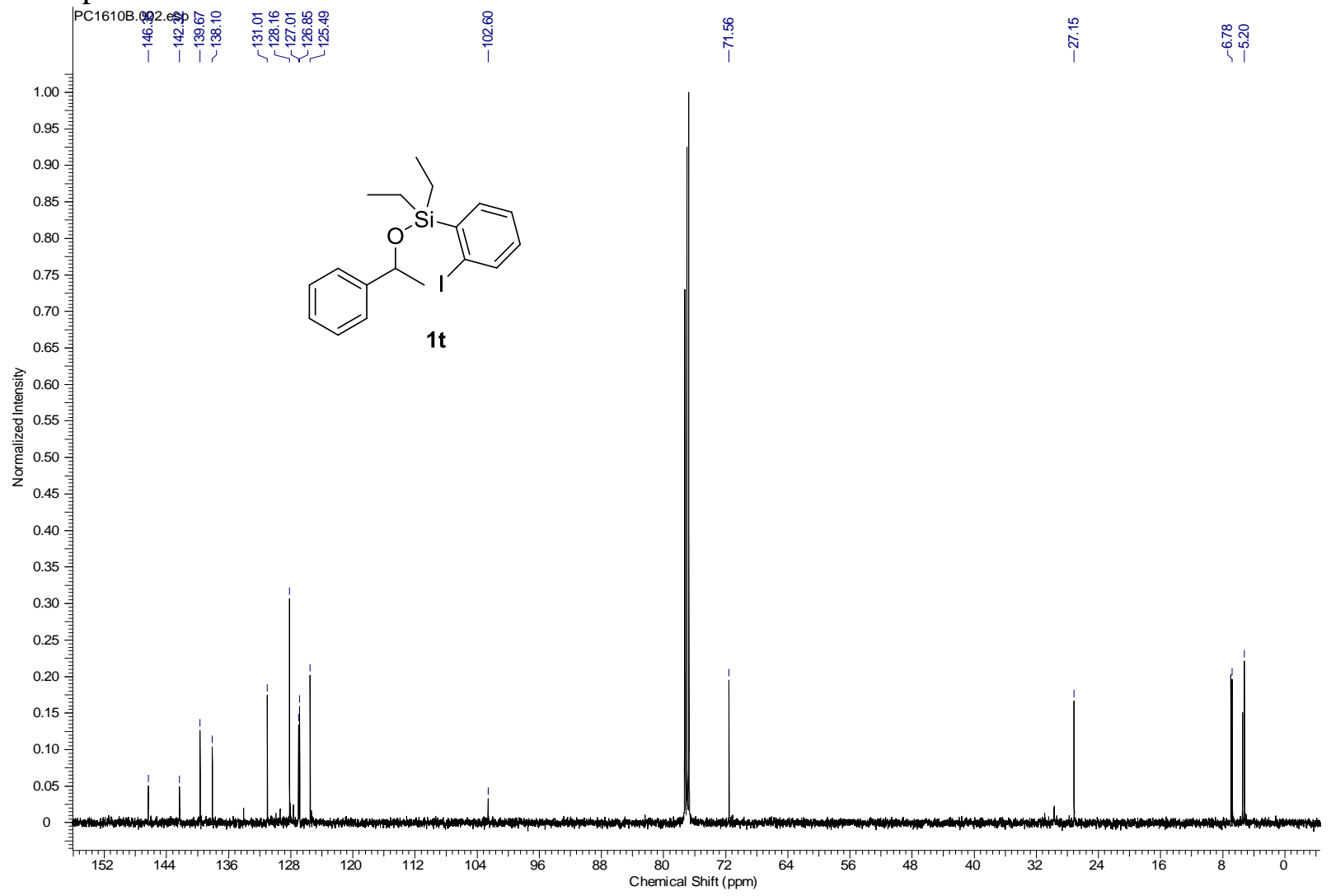




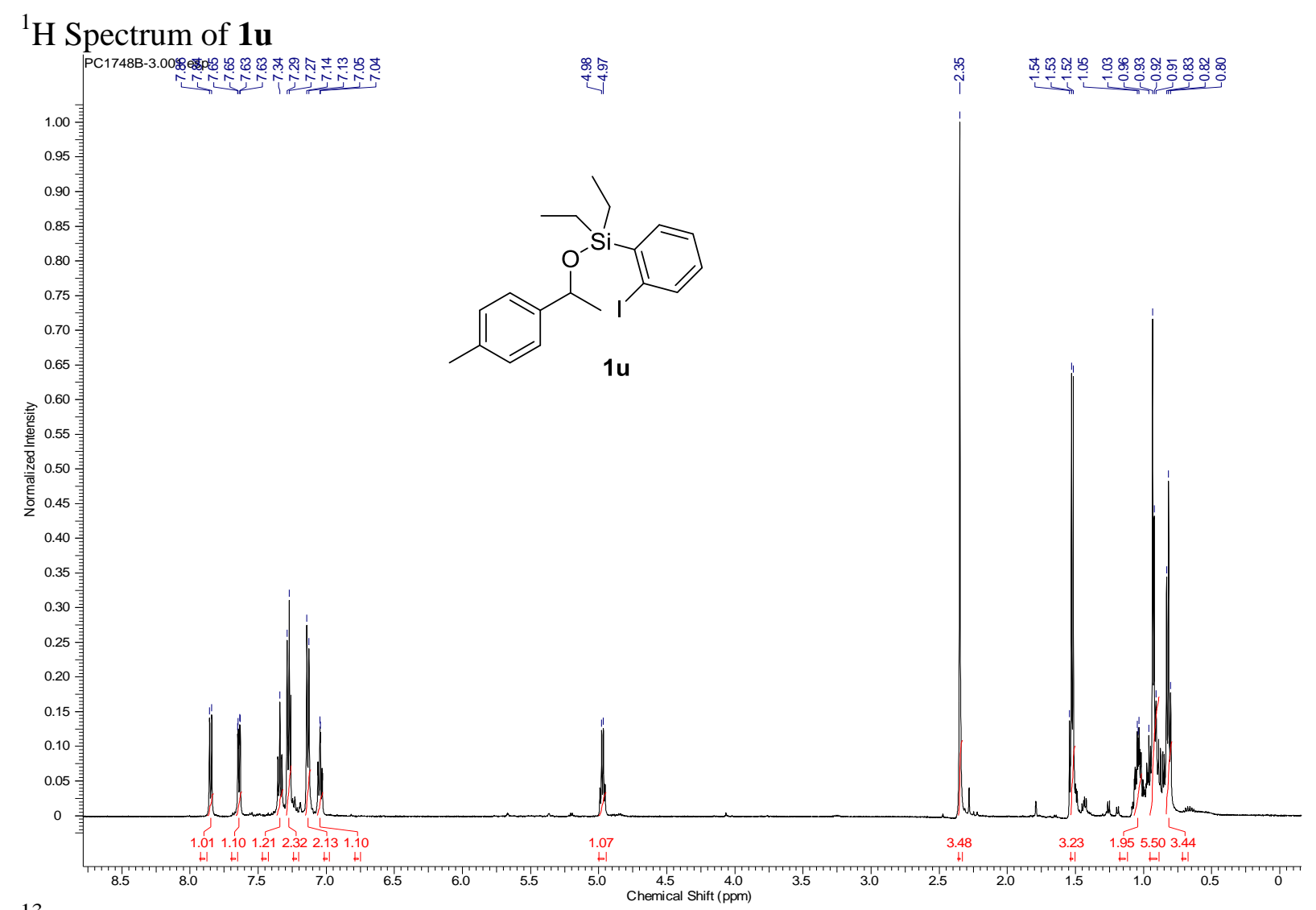

\section{${ }^{13} \mathrm{C}$ Spectrum of $\mathbf{1 u}$}

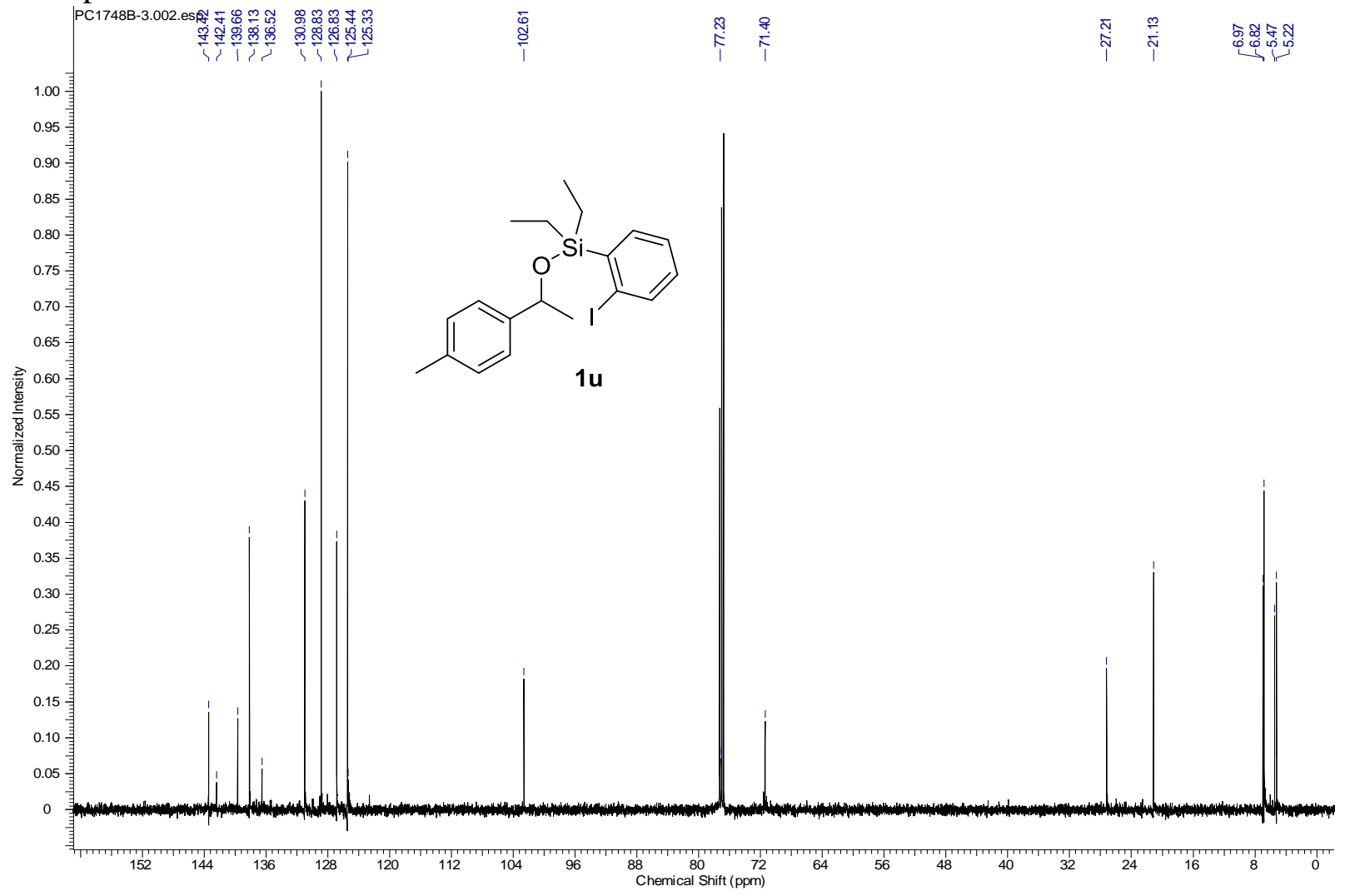




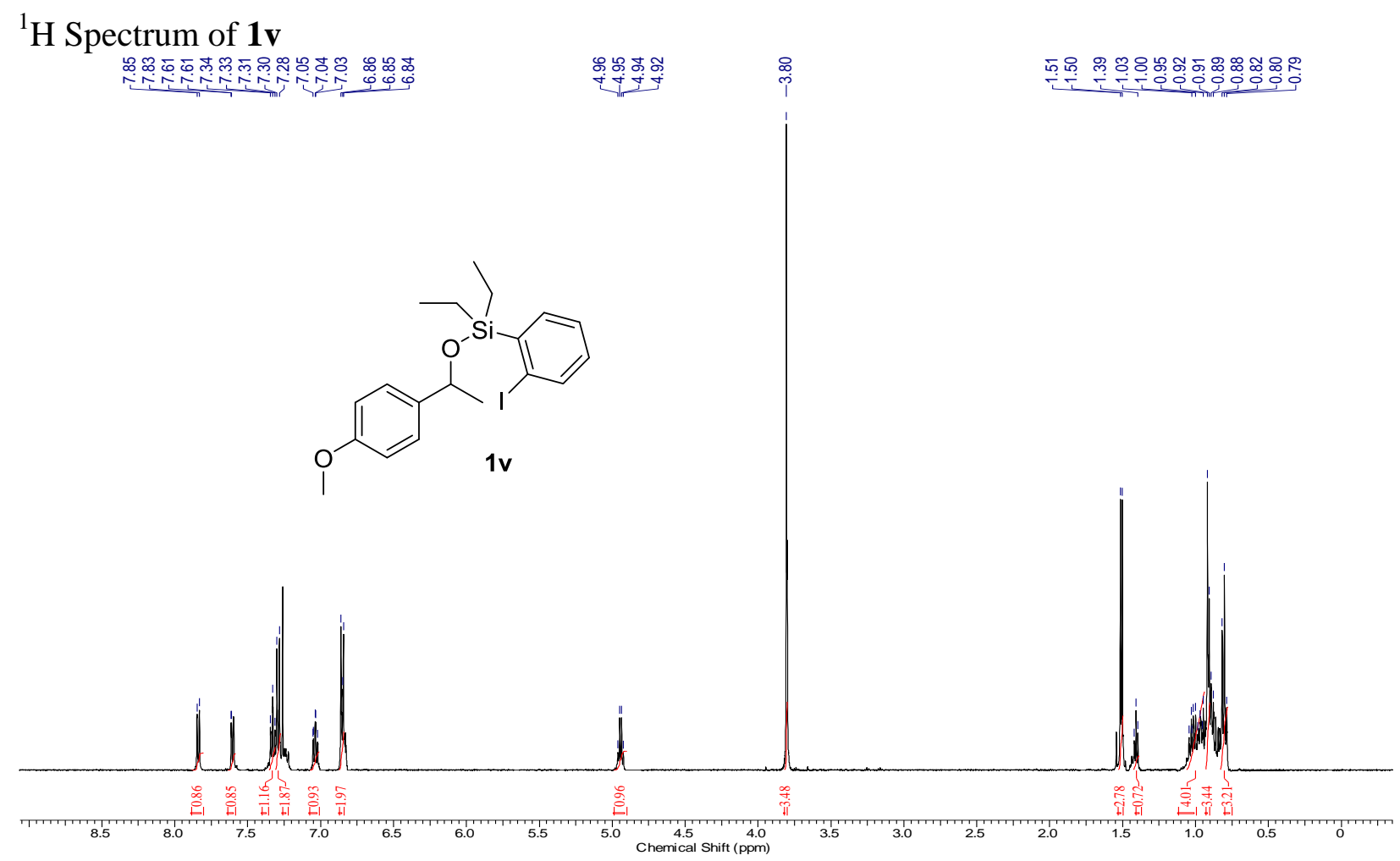

${ }^{13} \mathrm{C}$ Spectrum of $\mathbf{1 v}$

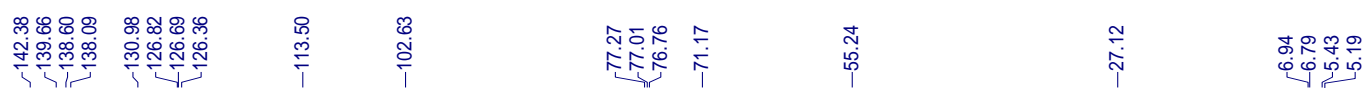

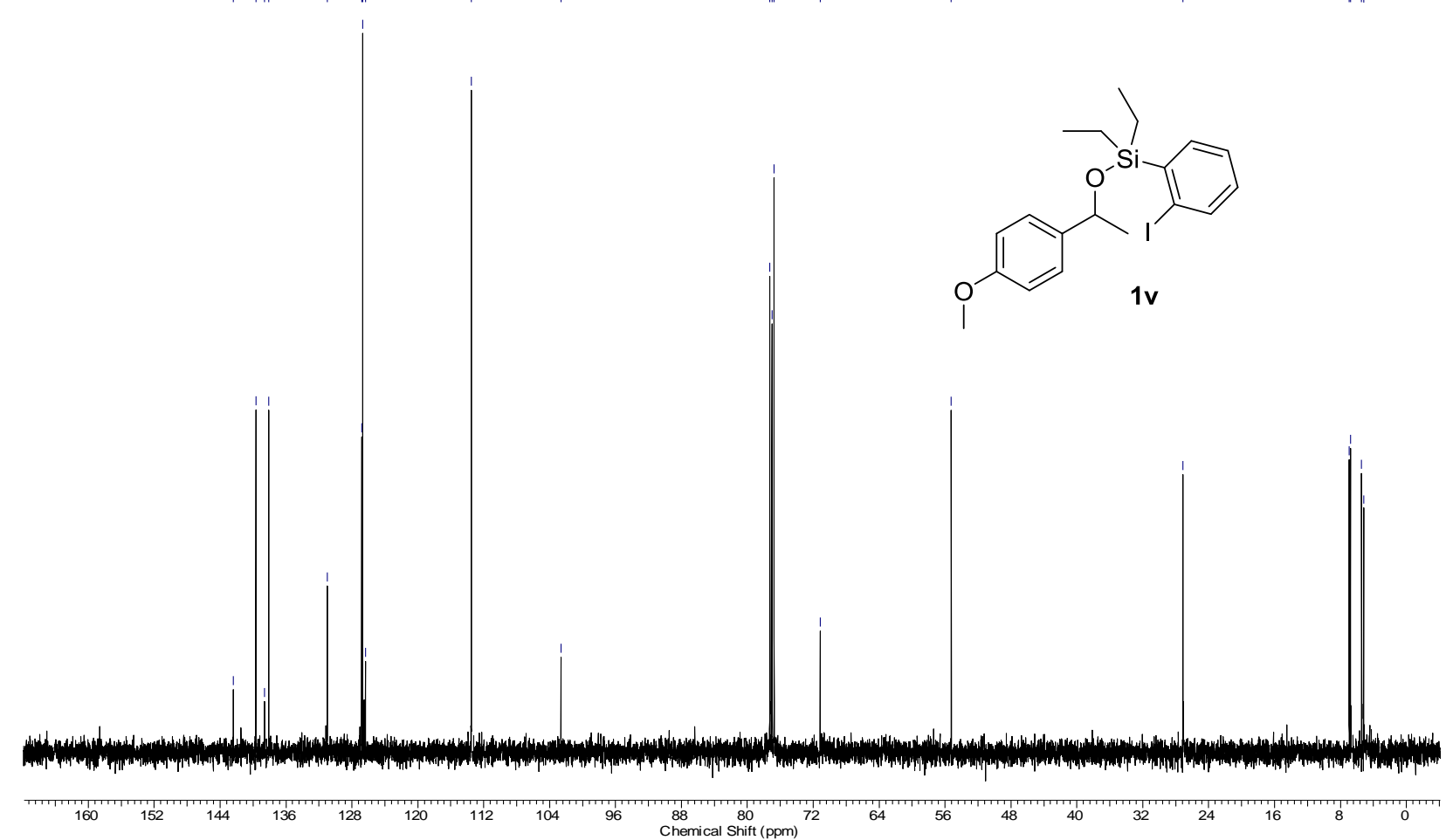




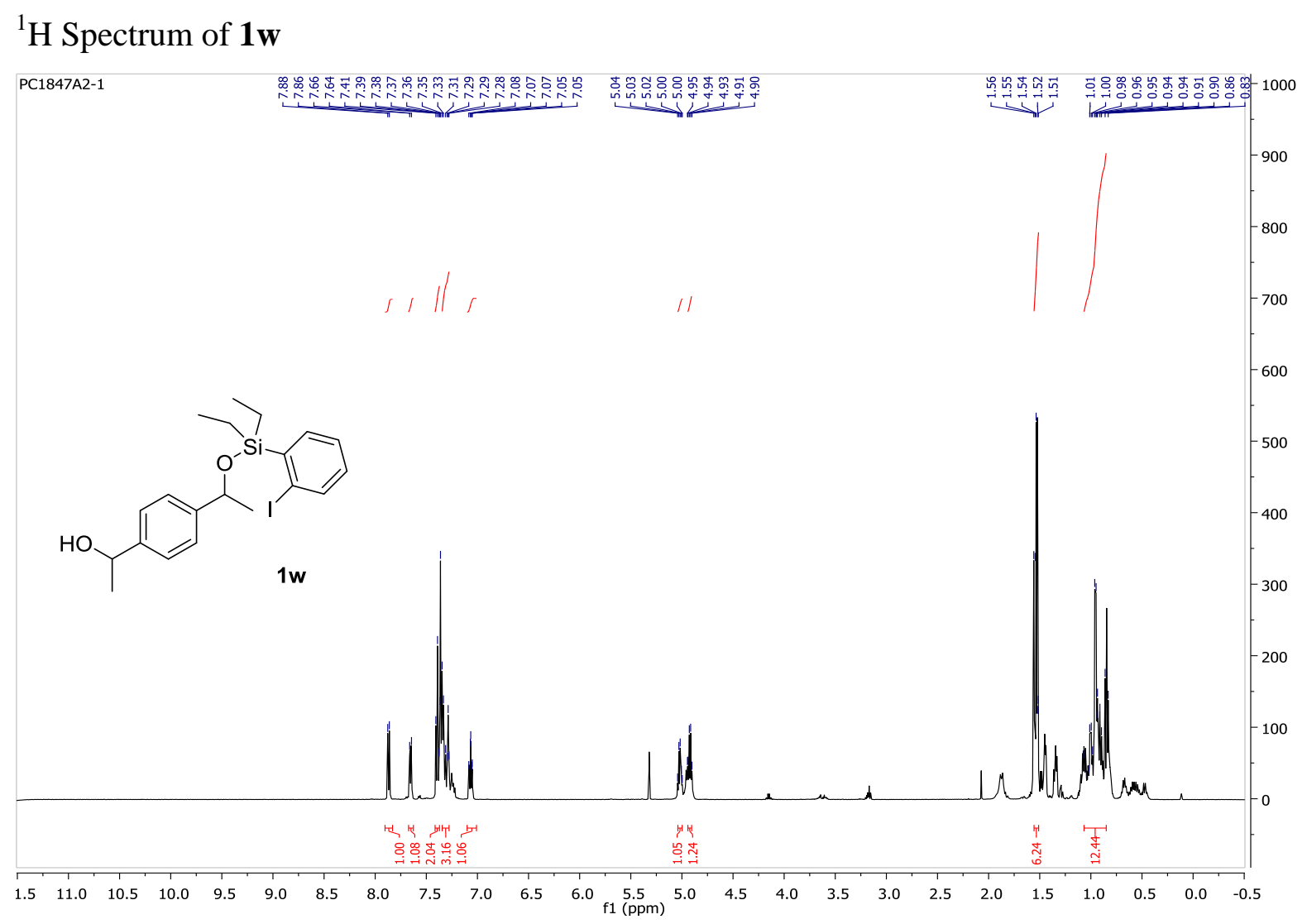

\section{${ }^{13} \mathrm{C}$ Spectrum of $\mathbf{1 w}$}

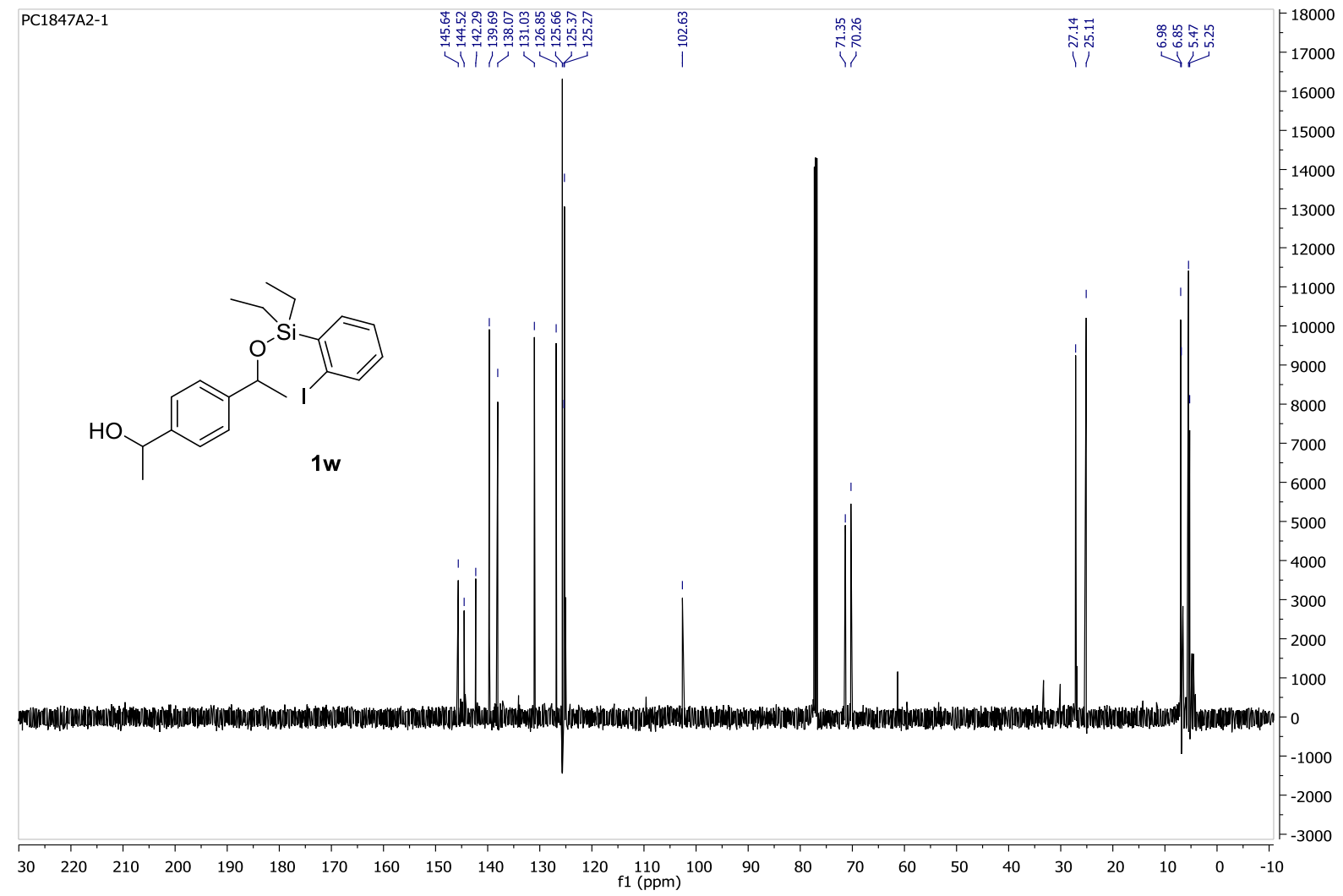




\section{${ }^{1} \mathrm{H}$ Spectrum of $\mathbf{1} \mathbf{x}$}

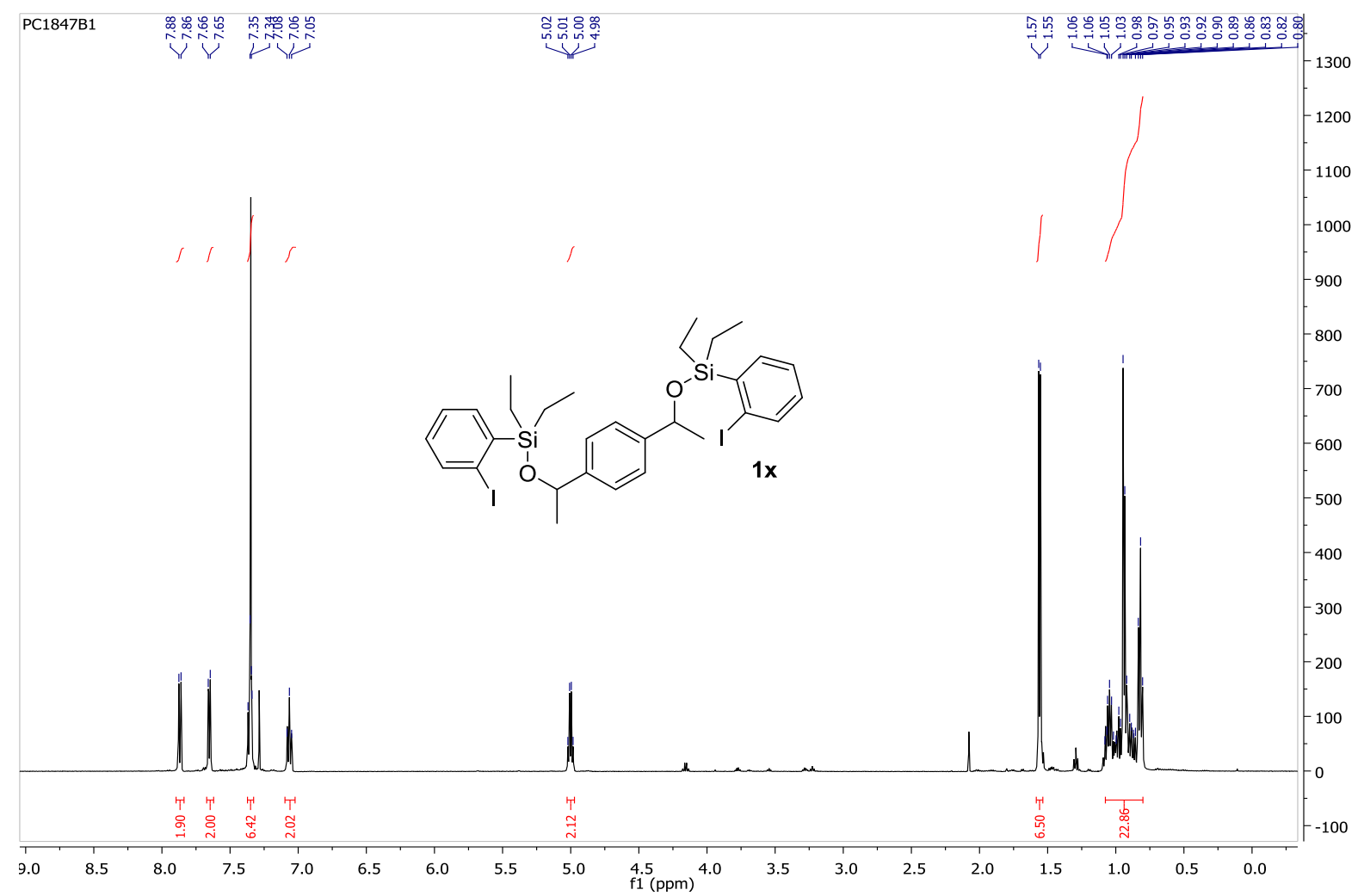

${ }^{13} \mathrm{C}$ Spectrum of $\mathbf{1 x}$

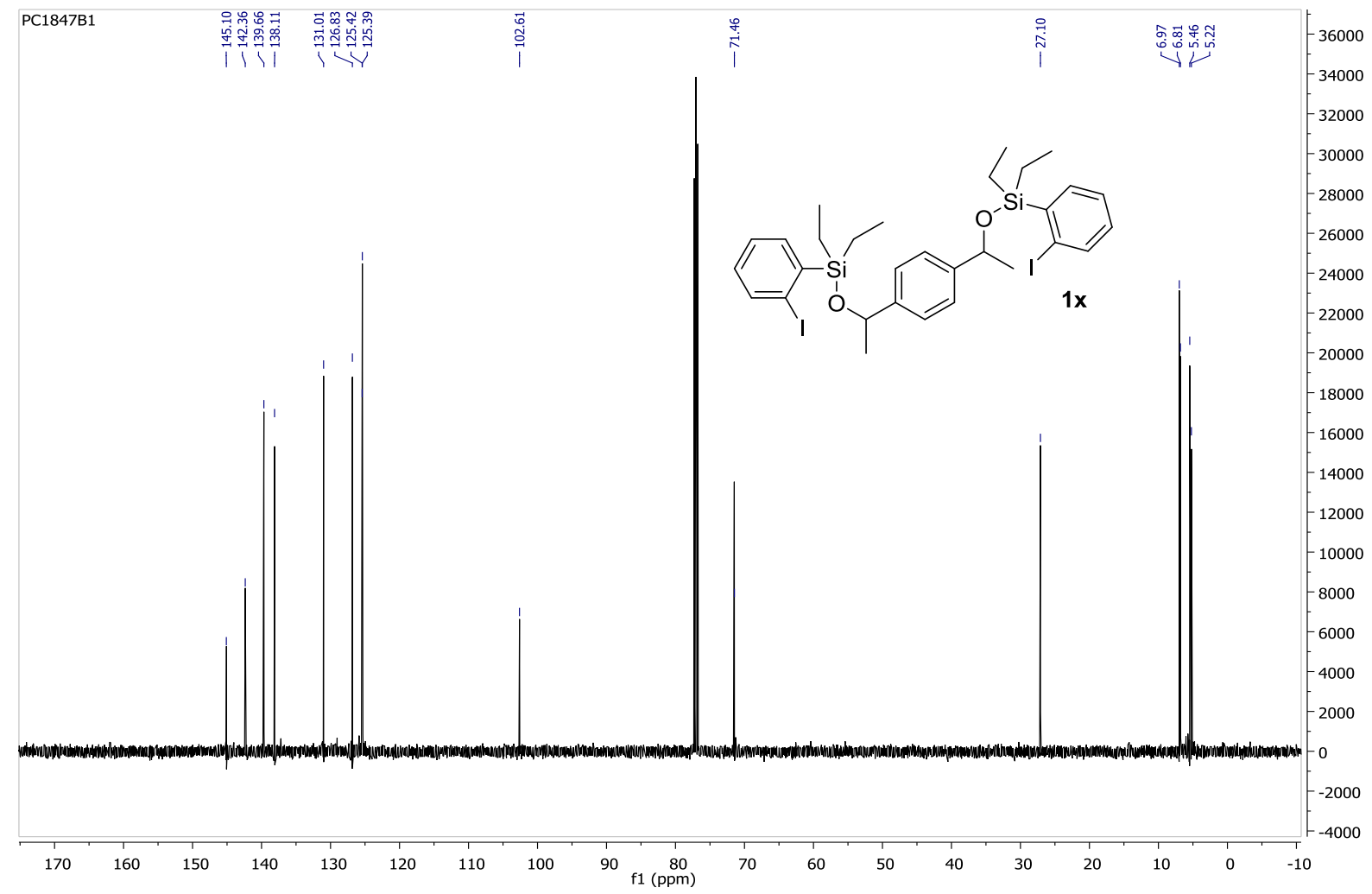




\section{${ }^{1} \mathrm{H}$ Spectrum of $\mathbf{1 y}$}

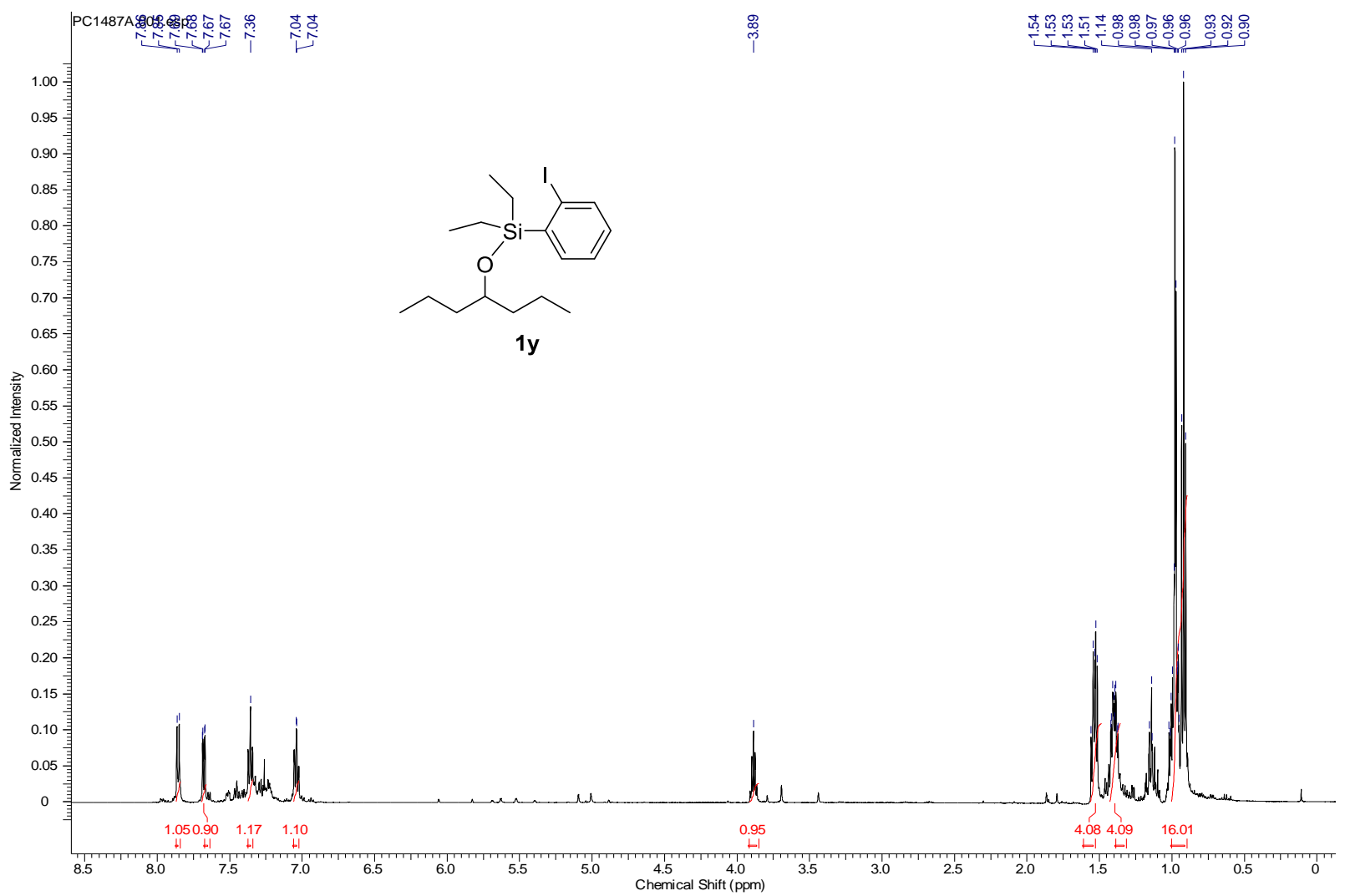

\section{${ }^{13} \mathrm{C}$ Spectrum of $\mathbf{1 y}$}

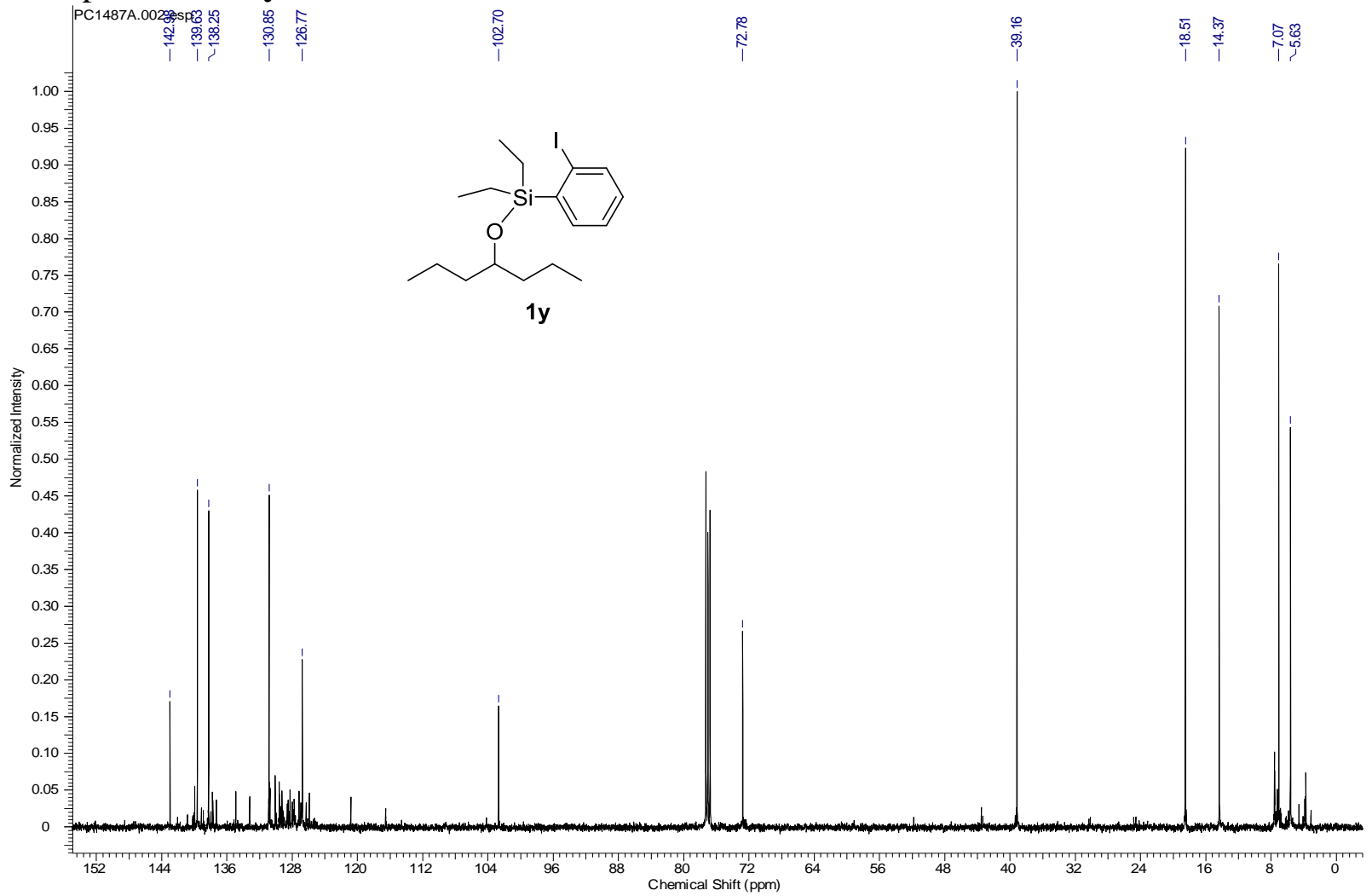




\section{${ }^{1} \mathrm{H}$ Spectrum of $\mathbf{1 z}$}

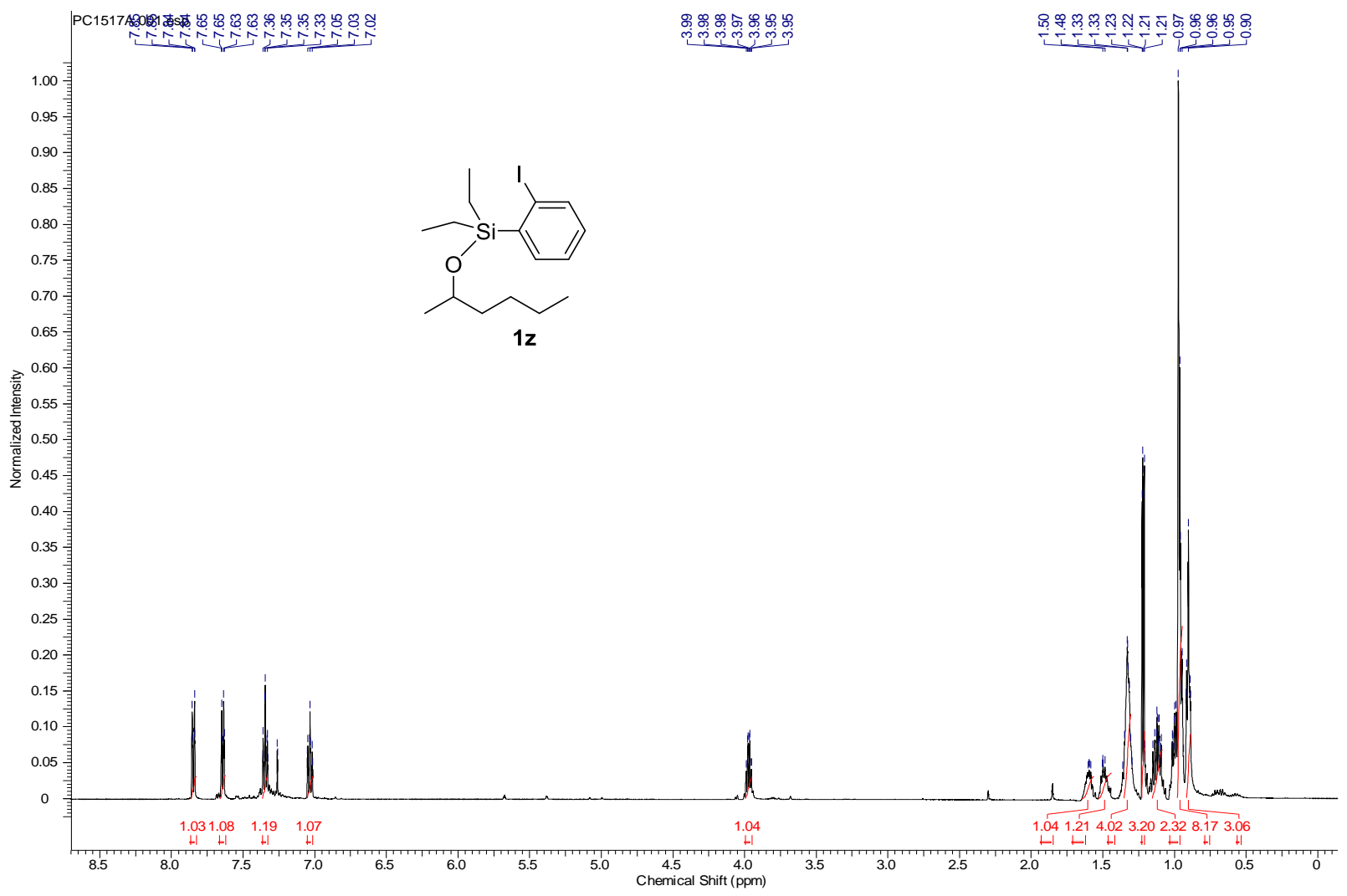

\section{${ }^{13} \mathrm{C}$ Spectrum of $\mathbf{1 z}$}

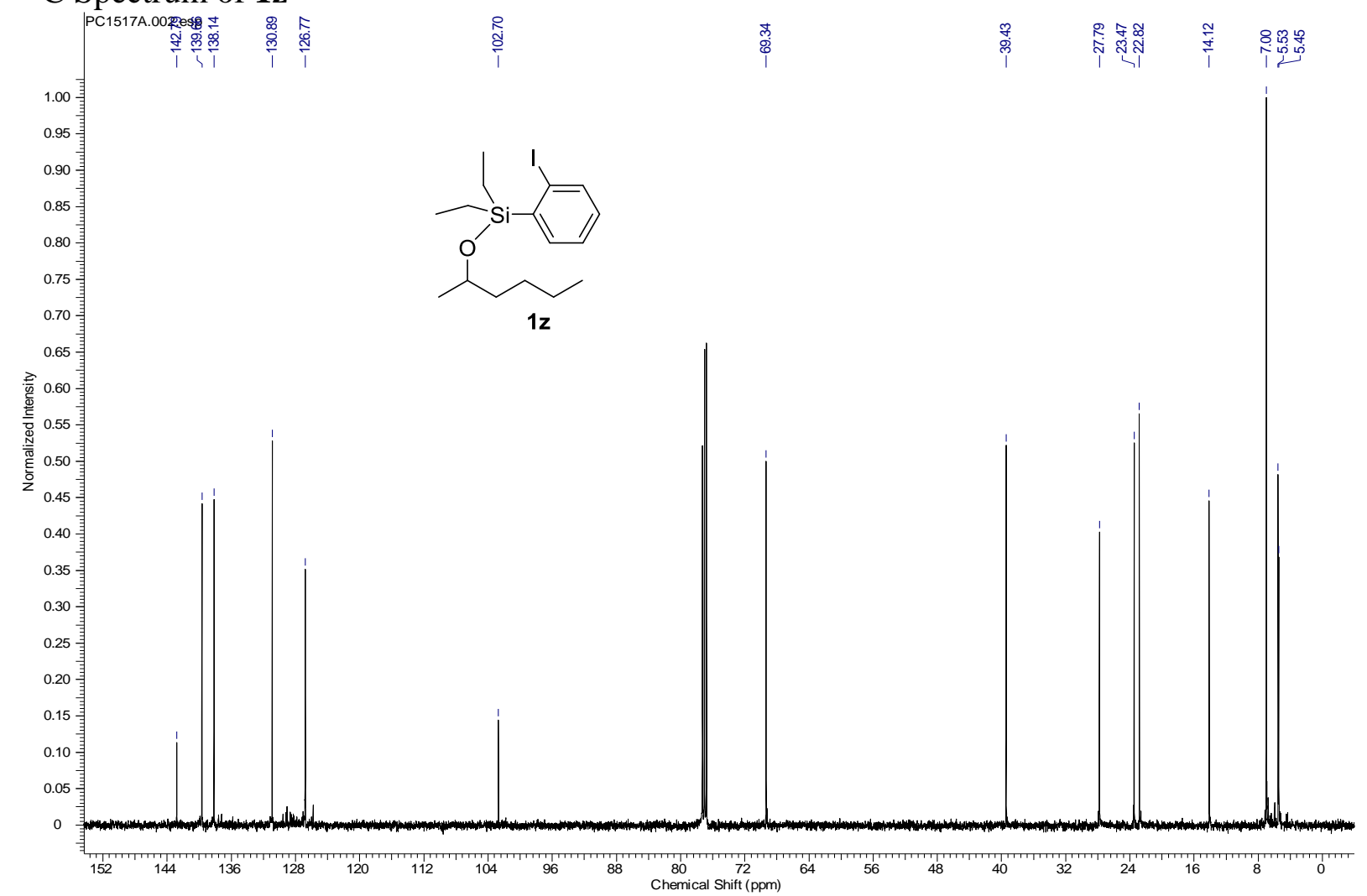


${ }^{1} \mathrm{H}$ Spectrum of 1 aa

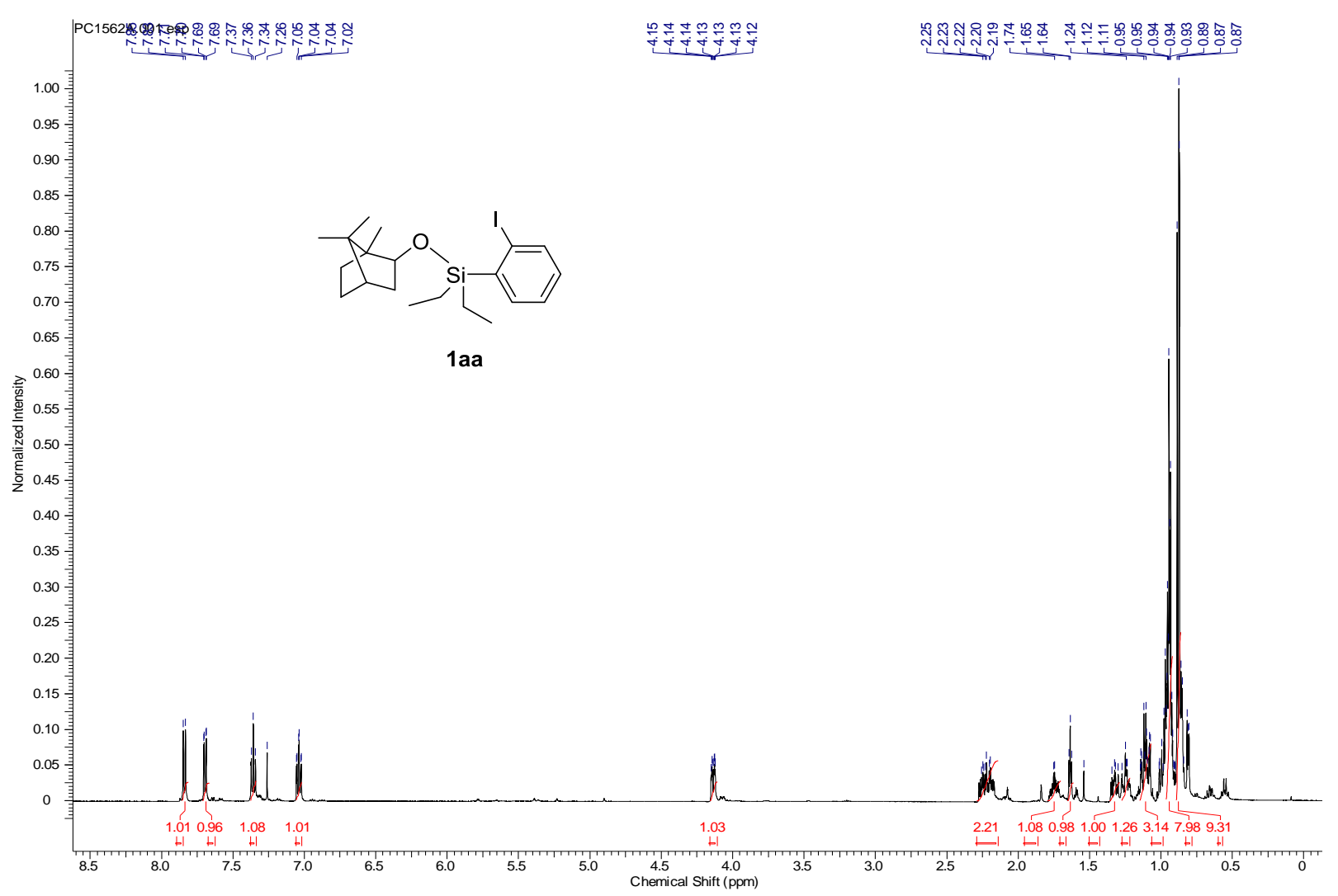

${ }^{13} \mathrm{C}$ Spectrum of $\mathbf{1 a a}$

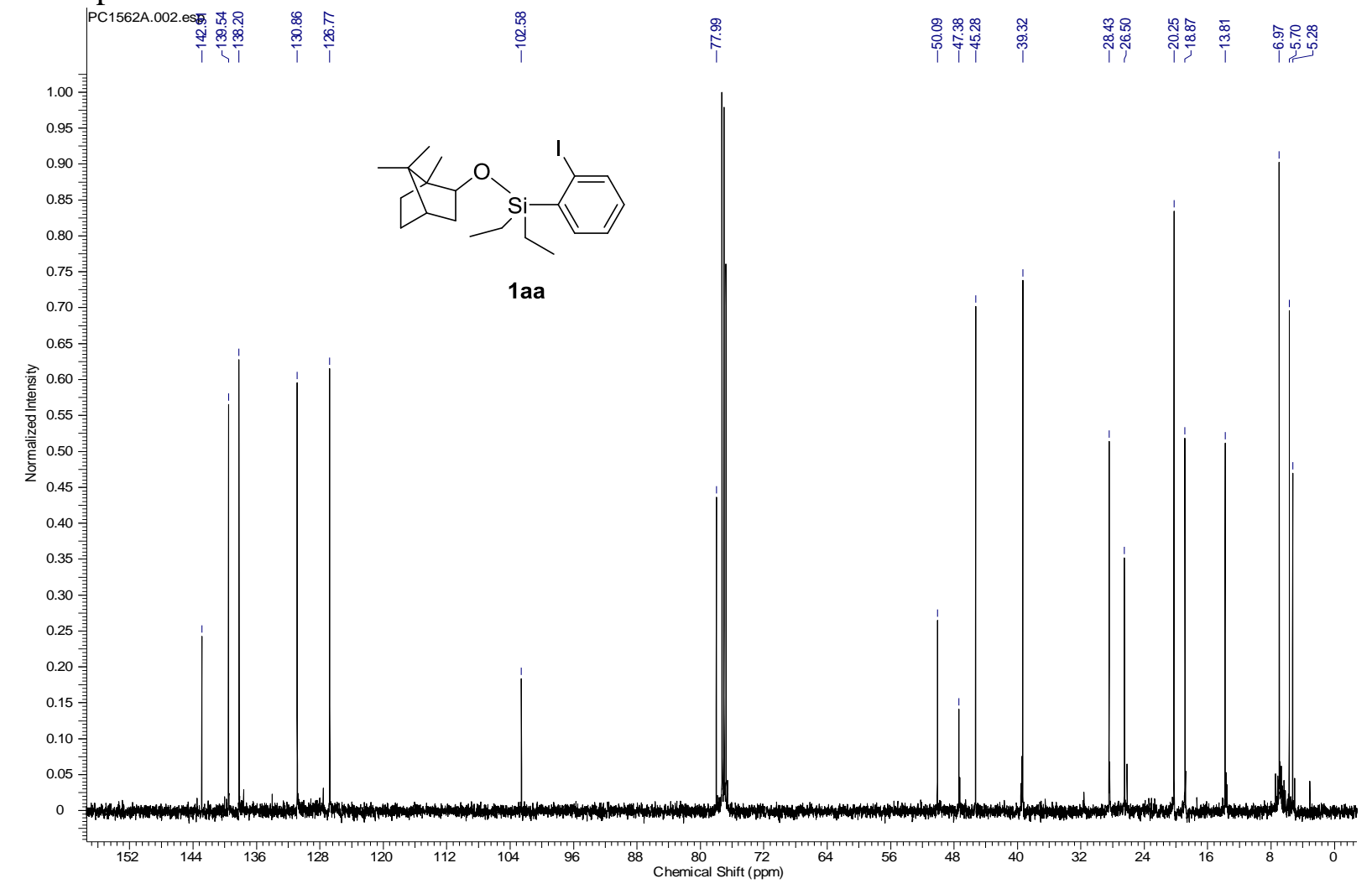




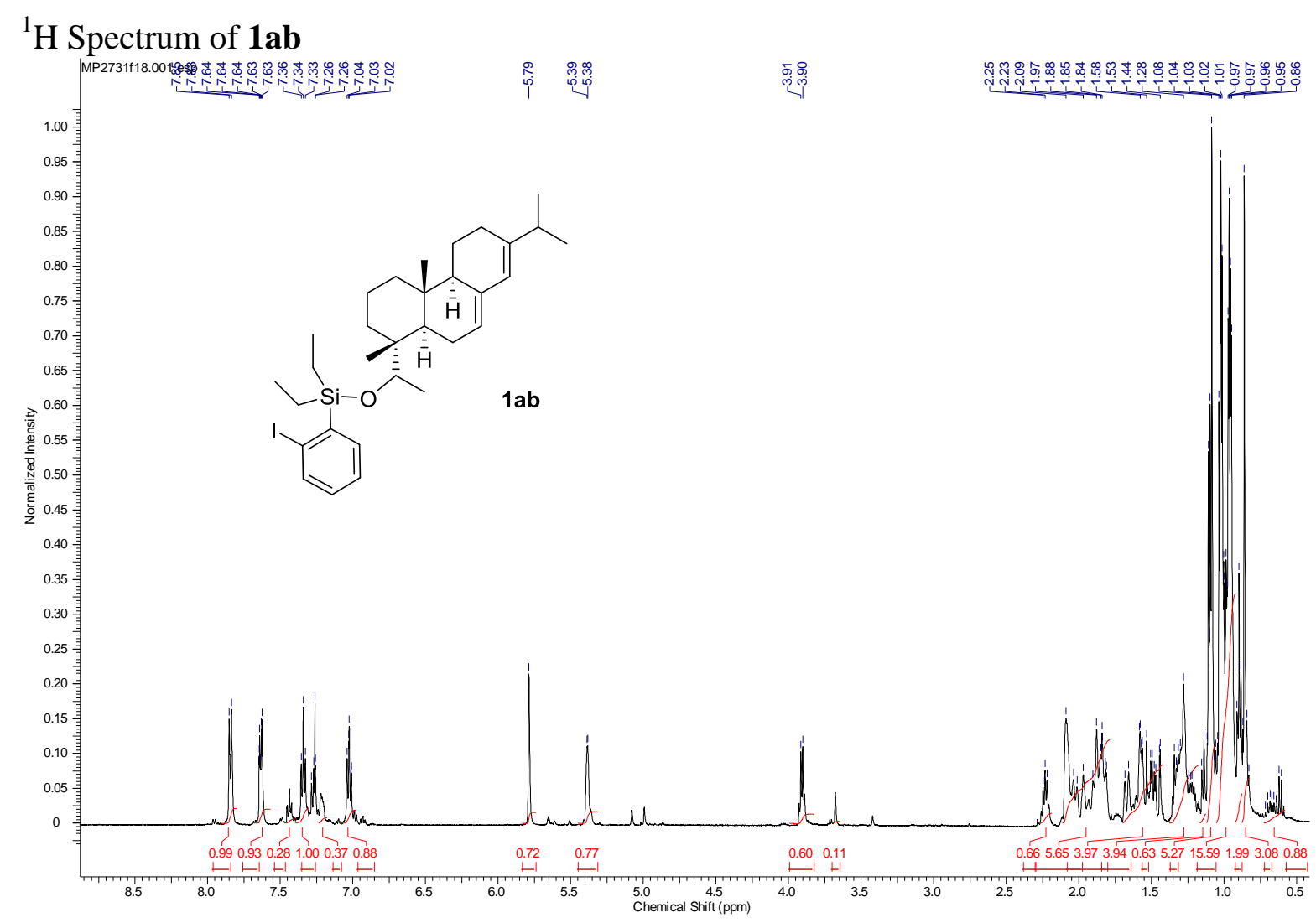

\section{${ }^{13} \mathrm{C}$ Spectrum of $\mathbf{1 a b}$}

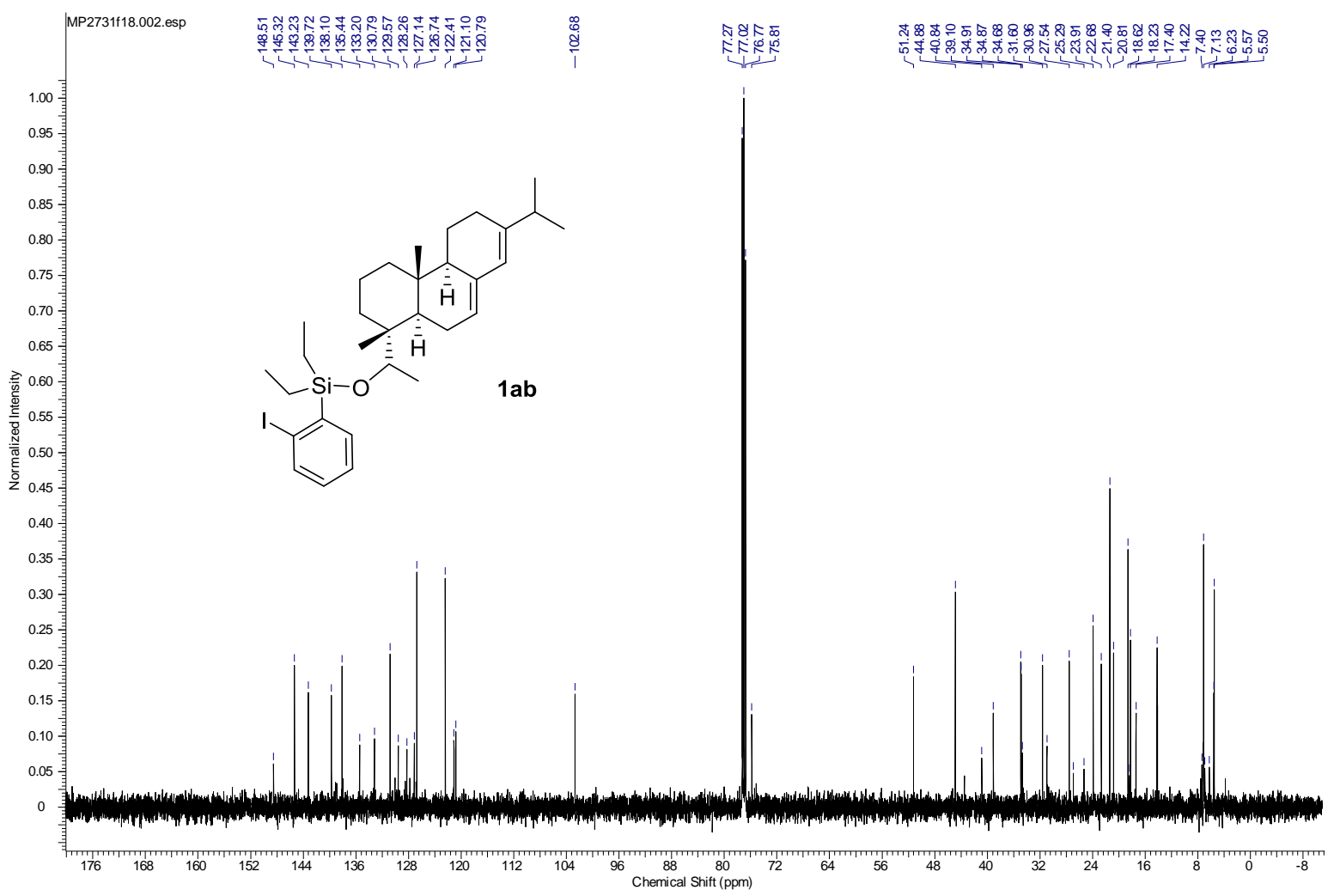




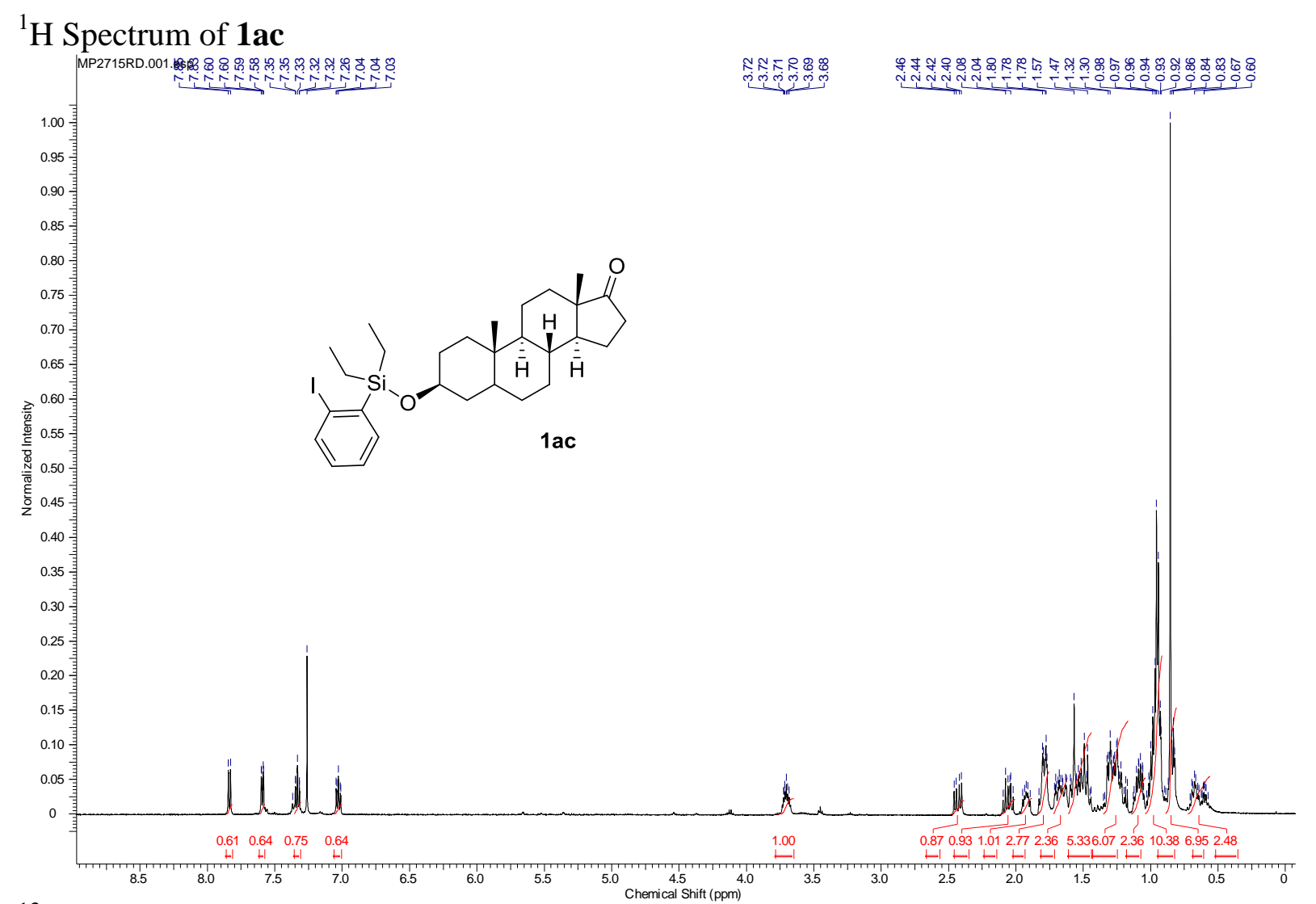

\section{${ }^{13} \mathrm{C}$ Spectrum of $\mathbf{1 a c}$}

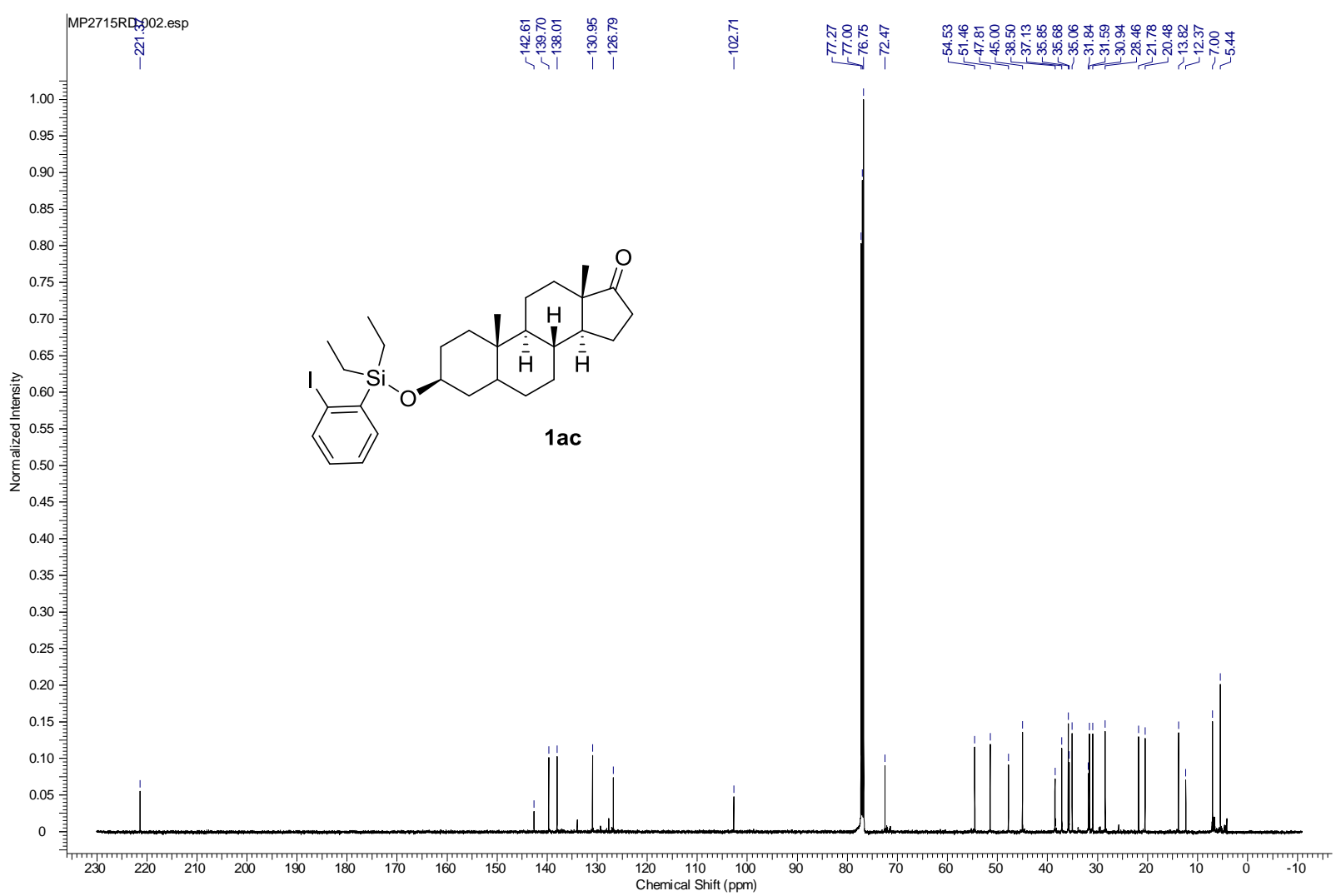




\section{${ }^{1} \mathrm{H}$ Spectrum of $\mathbf{2 a}$}

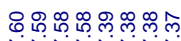

NNNNNN

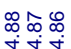

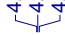

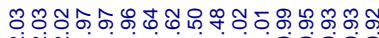

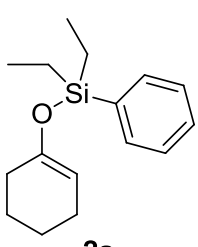

$2 a$

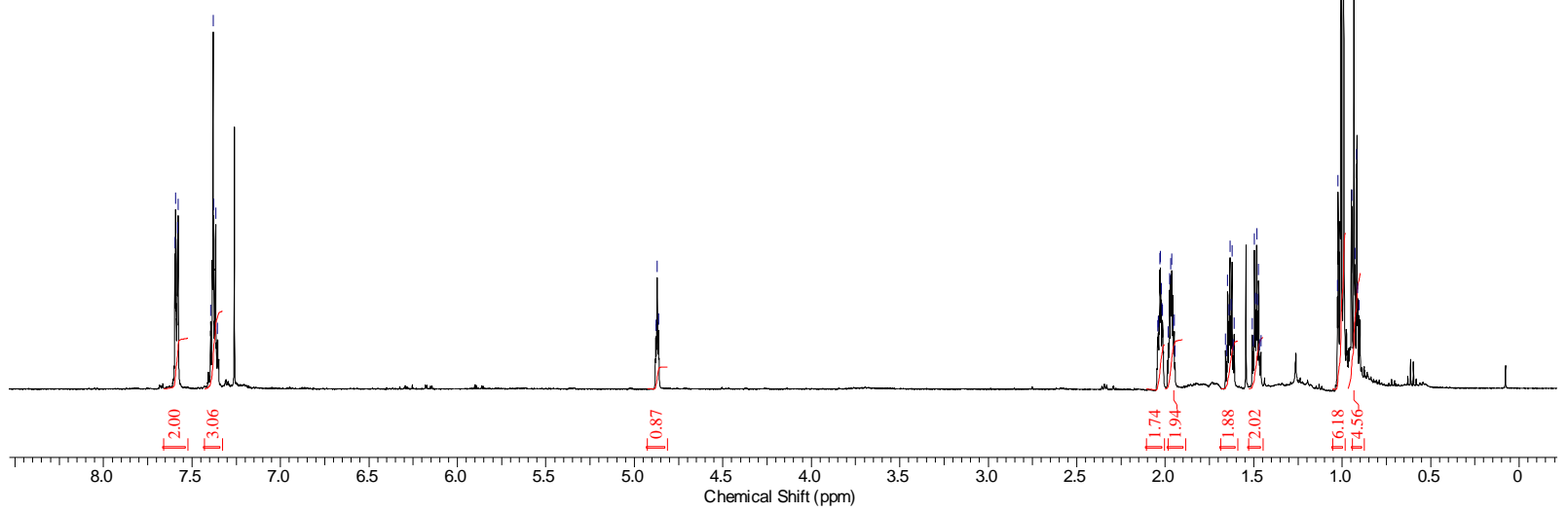

${ }^{13} \mathrm{C}$ Spectrum of $\mathbf{2 a}$

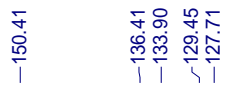
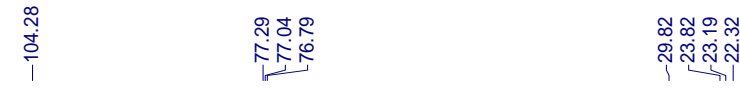

זio
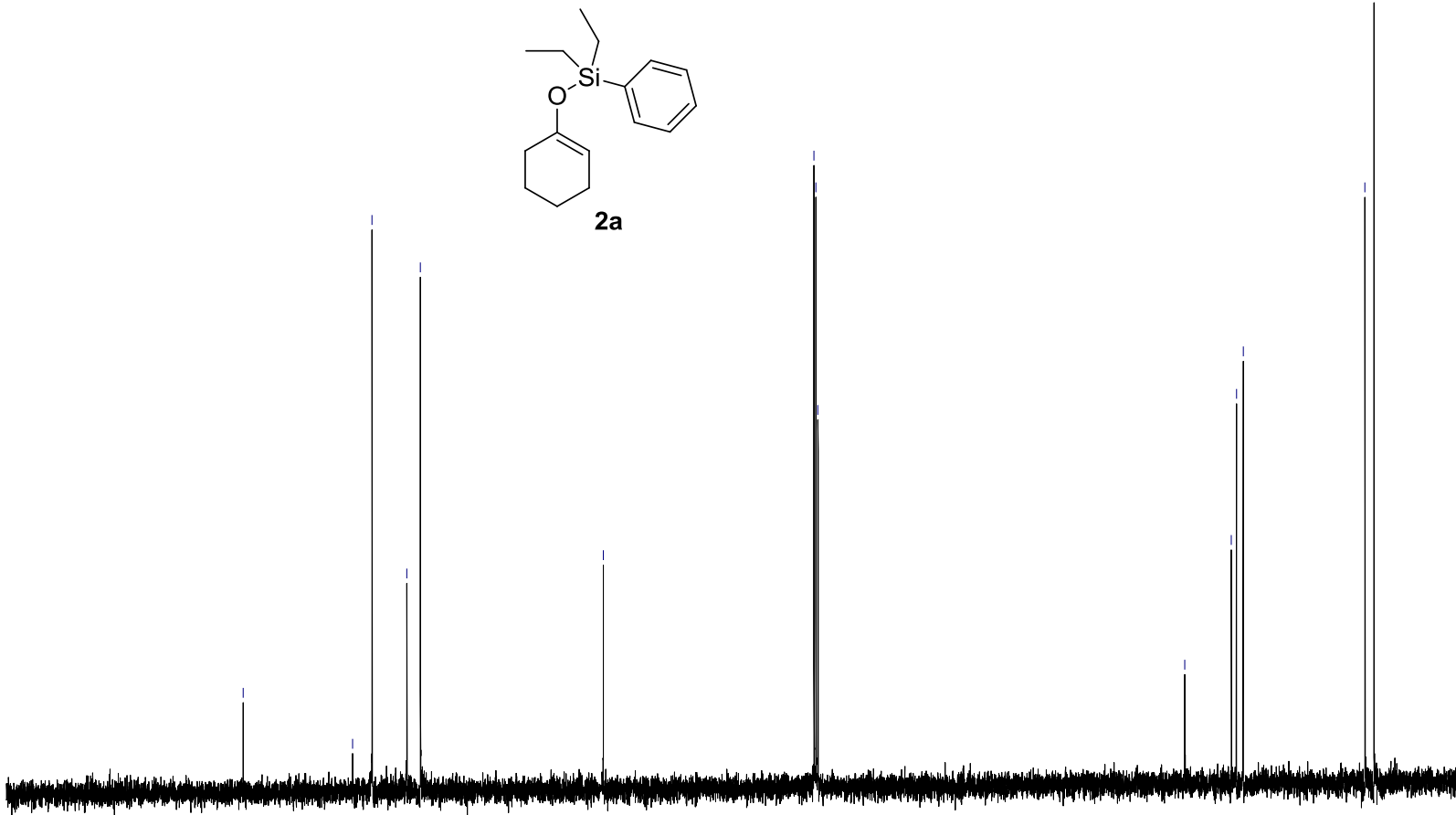

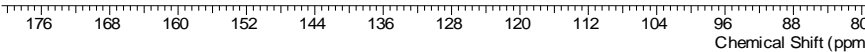




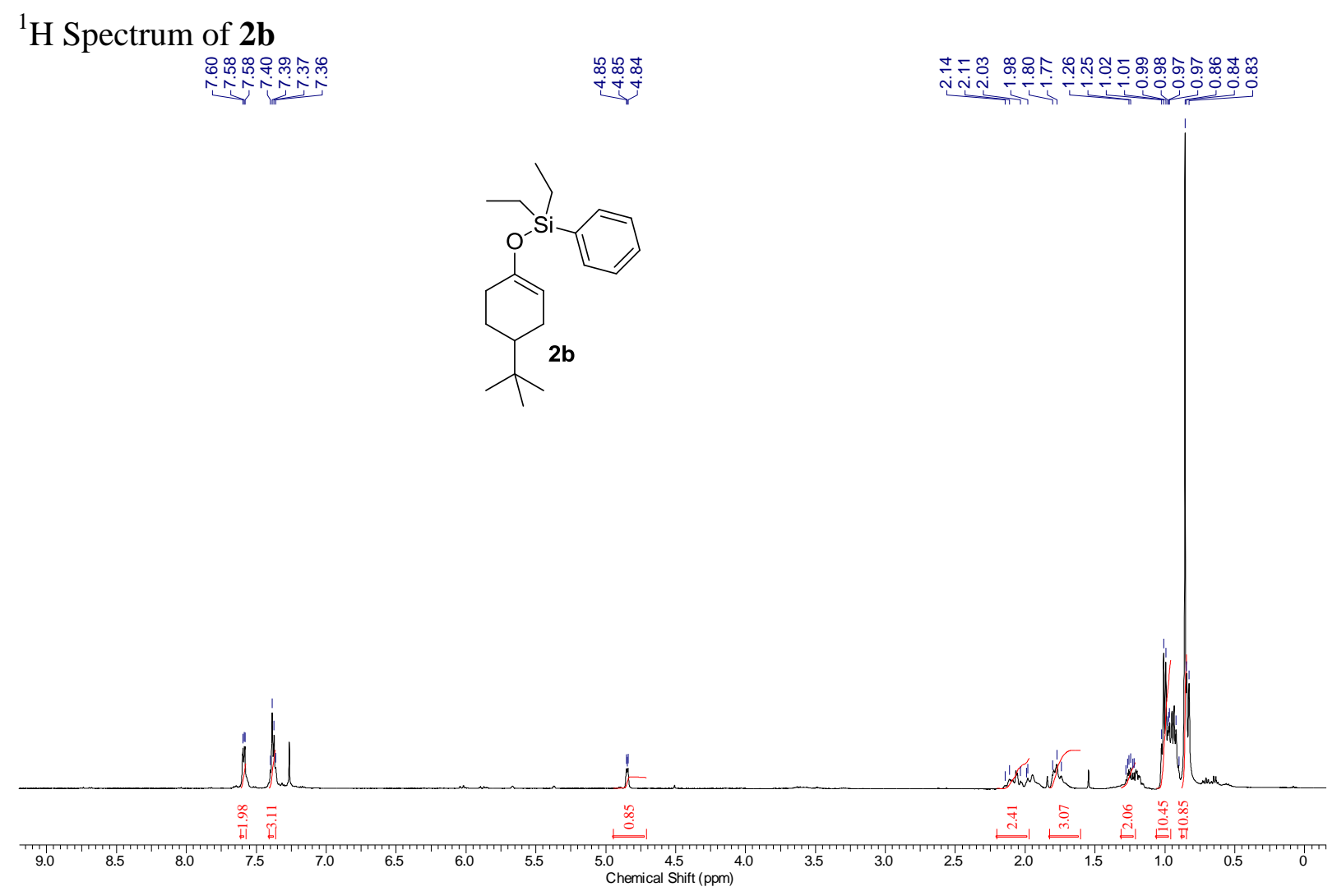

${ }^{13} \mathrm{C}$ Spectrum of $\mathbf{2 b}$

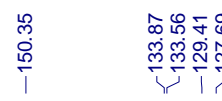

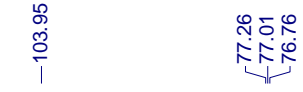
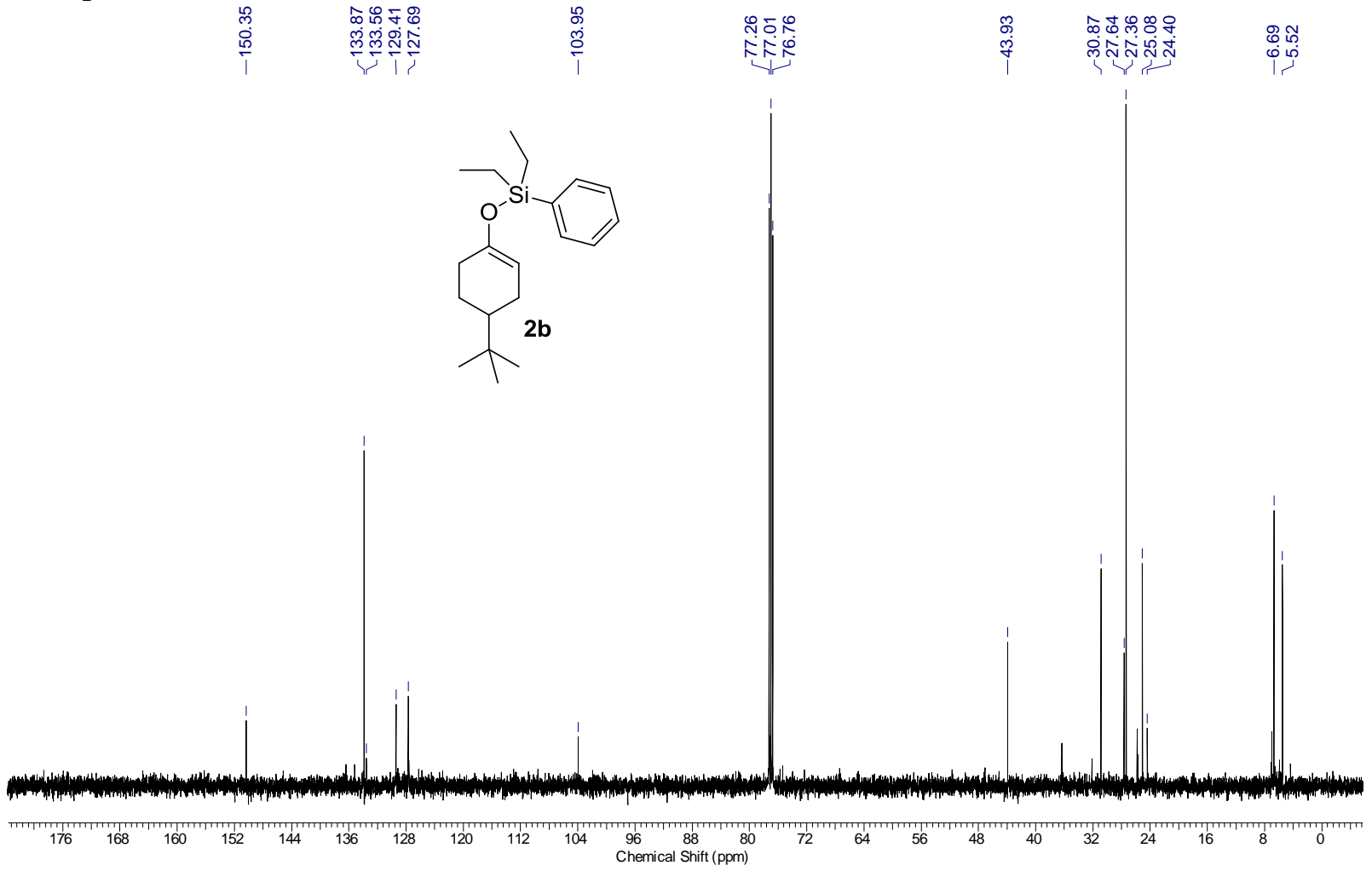


\section{${ }^{1} \mathrm{H}$ Spectrum of $\mathbf{2 c}$}

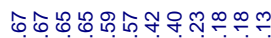

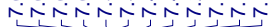

$$
\text { mำ }
$$

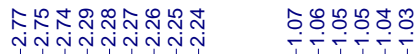<smiles>CC[Si](CC)(OC1=CCCc2ccccc21)c1ccccc1</smiles>

2c

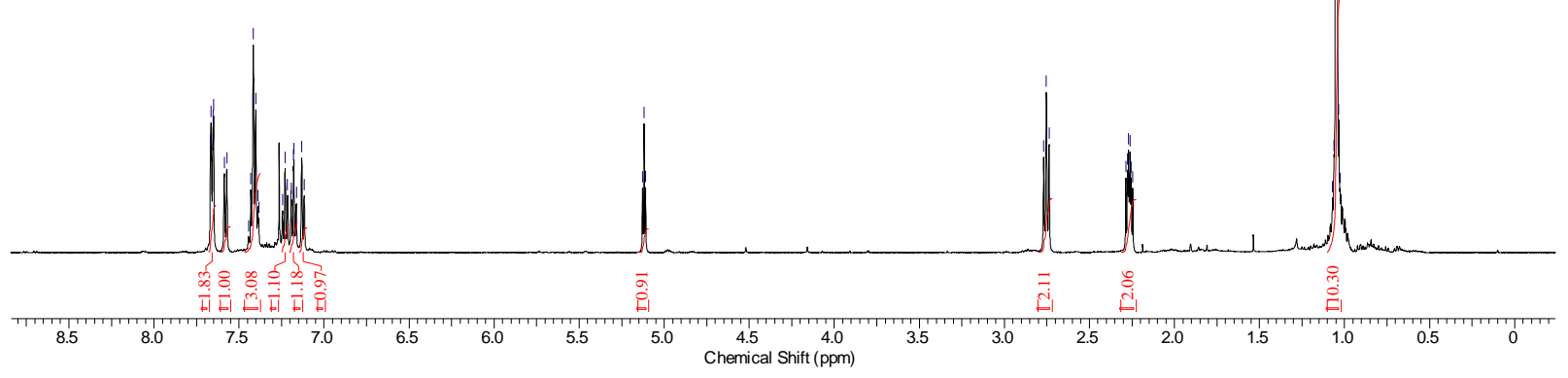

${ }^{13} \mathrm{C}$ Spectrum of $\mathbf{2 c}$
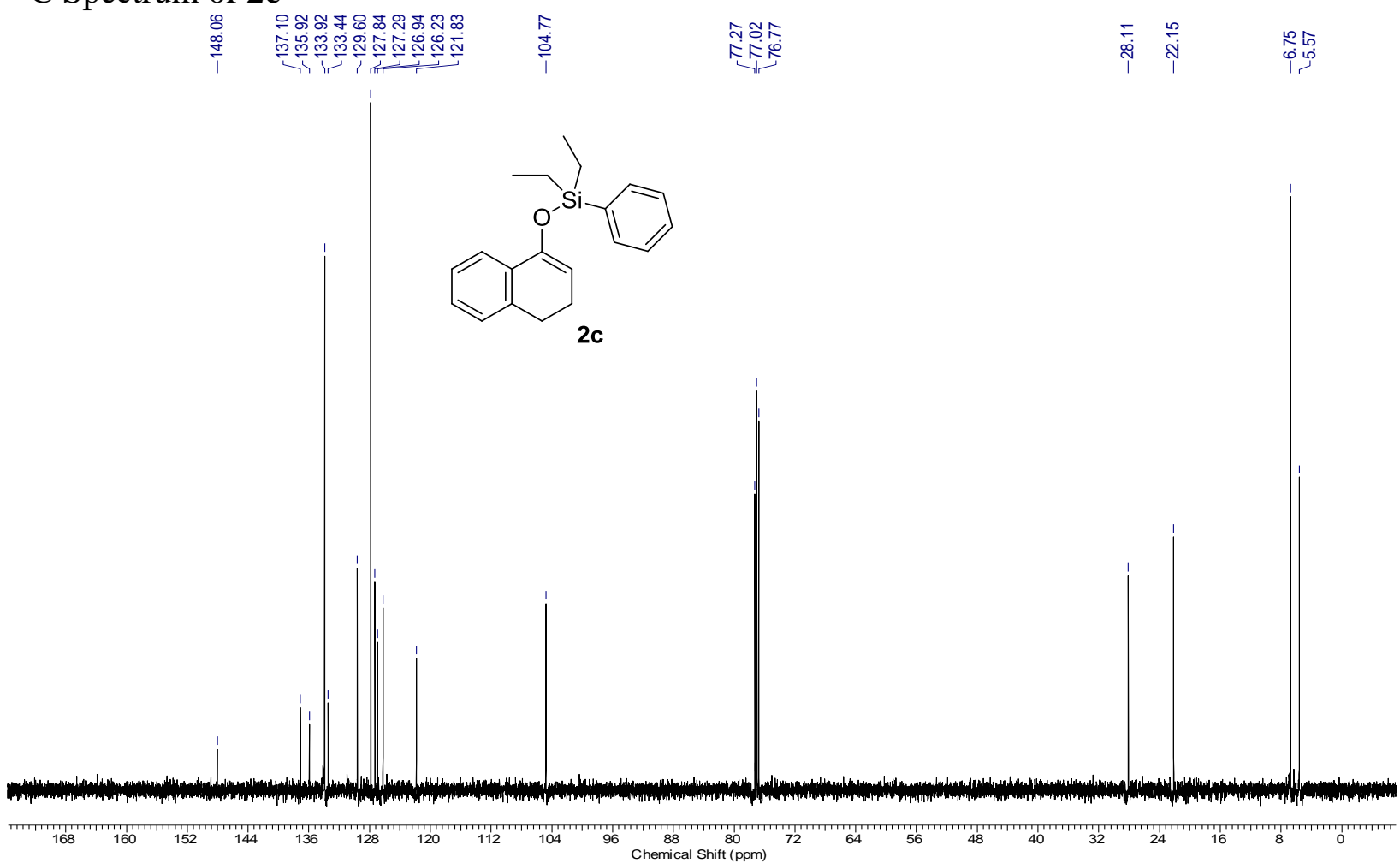
${ }^{1} \mathrm{H}$ Spectrum of $\mathbf{2 d}$

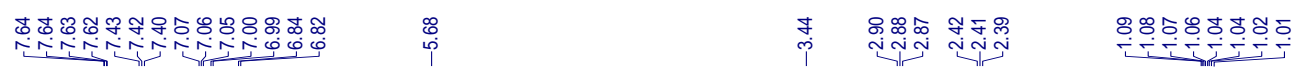<smiles>CC[Si](OC)(OC1=Cc2ccccc2C1)c1ccccc1</smiles>

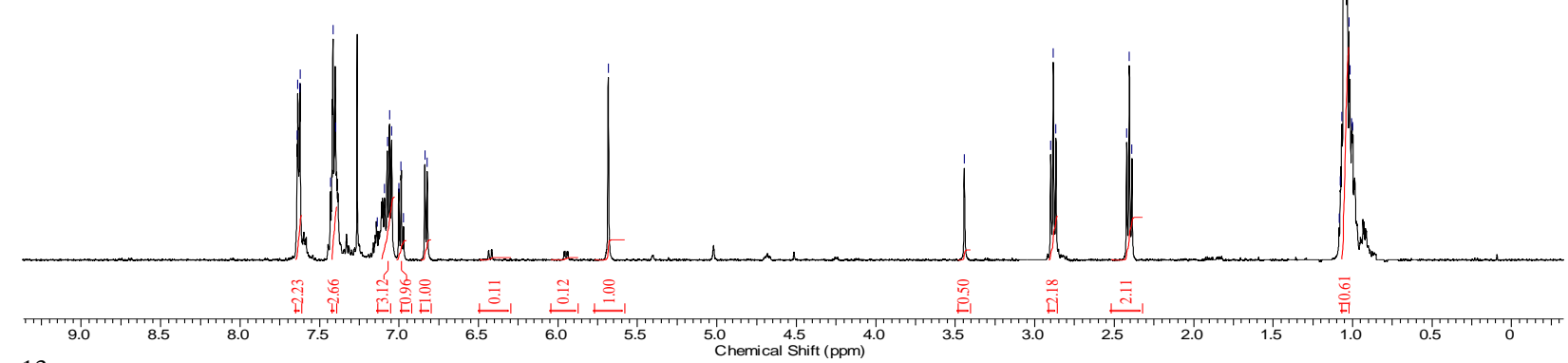

${ }^{13} \mathrm{C}$ Spectrum of $\mathbf{2 d}$

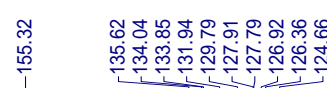

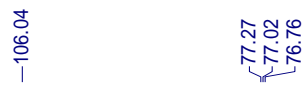

¿̊m

요요용

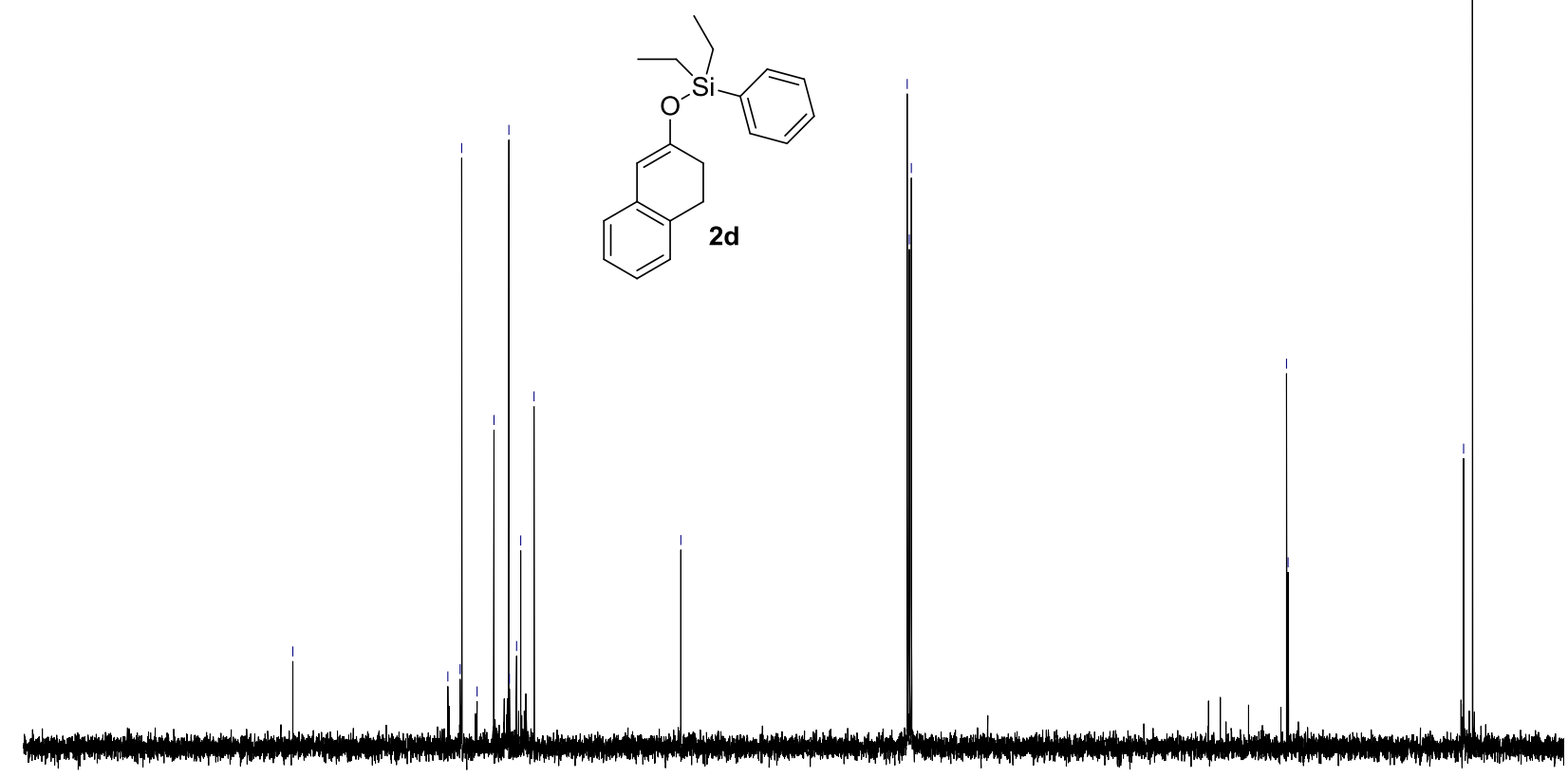

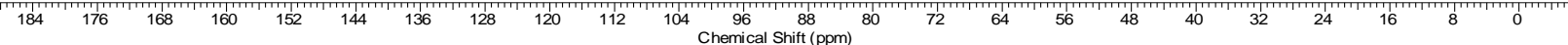




\section{${ }^{1} \mathrm{H}$ Spectrum of $\mathbf{2 e}$}

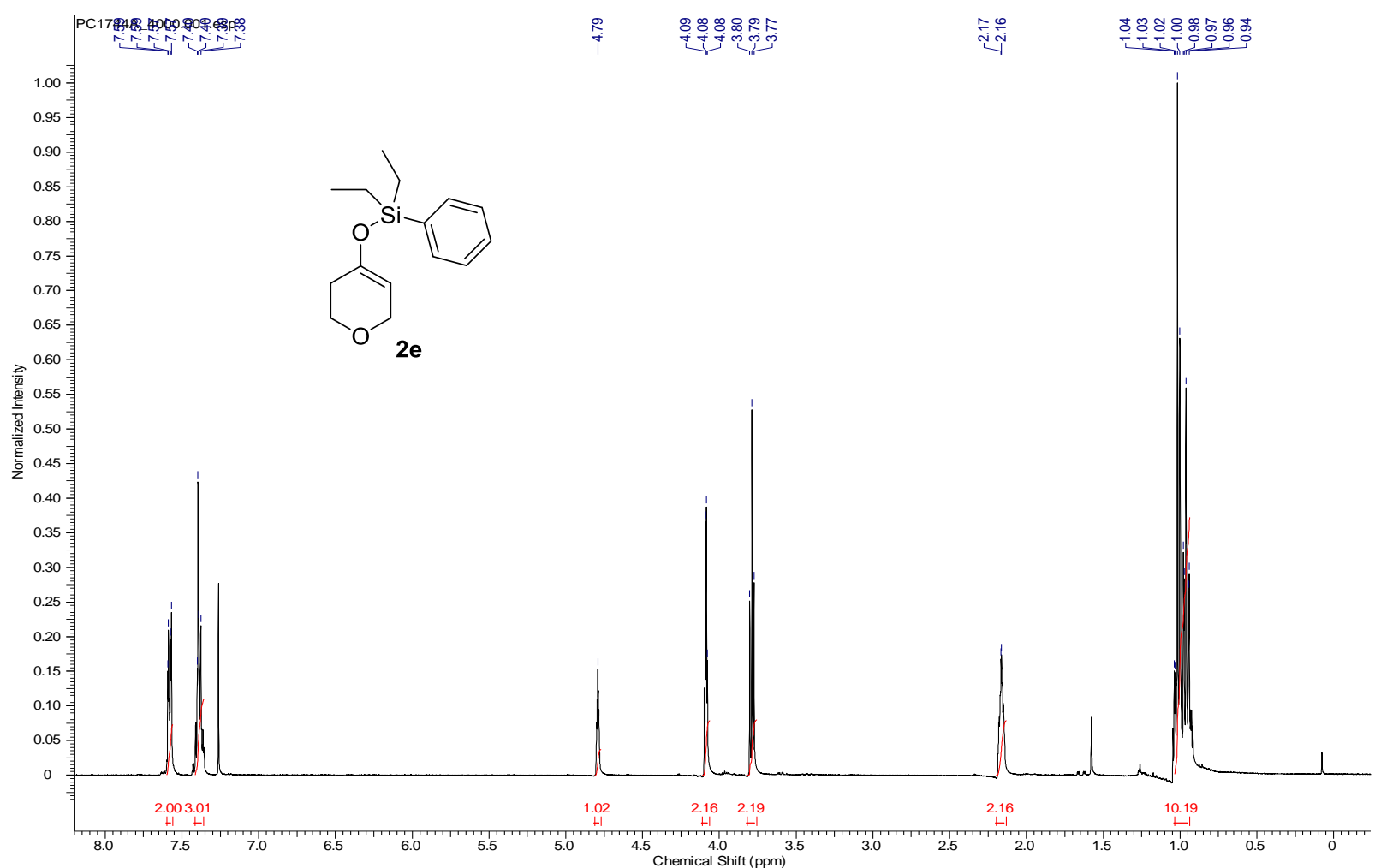

${ }^{13} \mathrm{C}$ Spectrum of $\mathbf{2 e}$

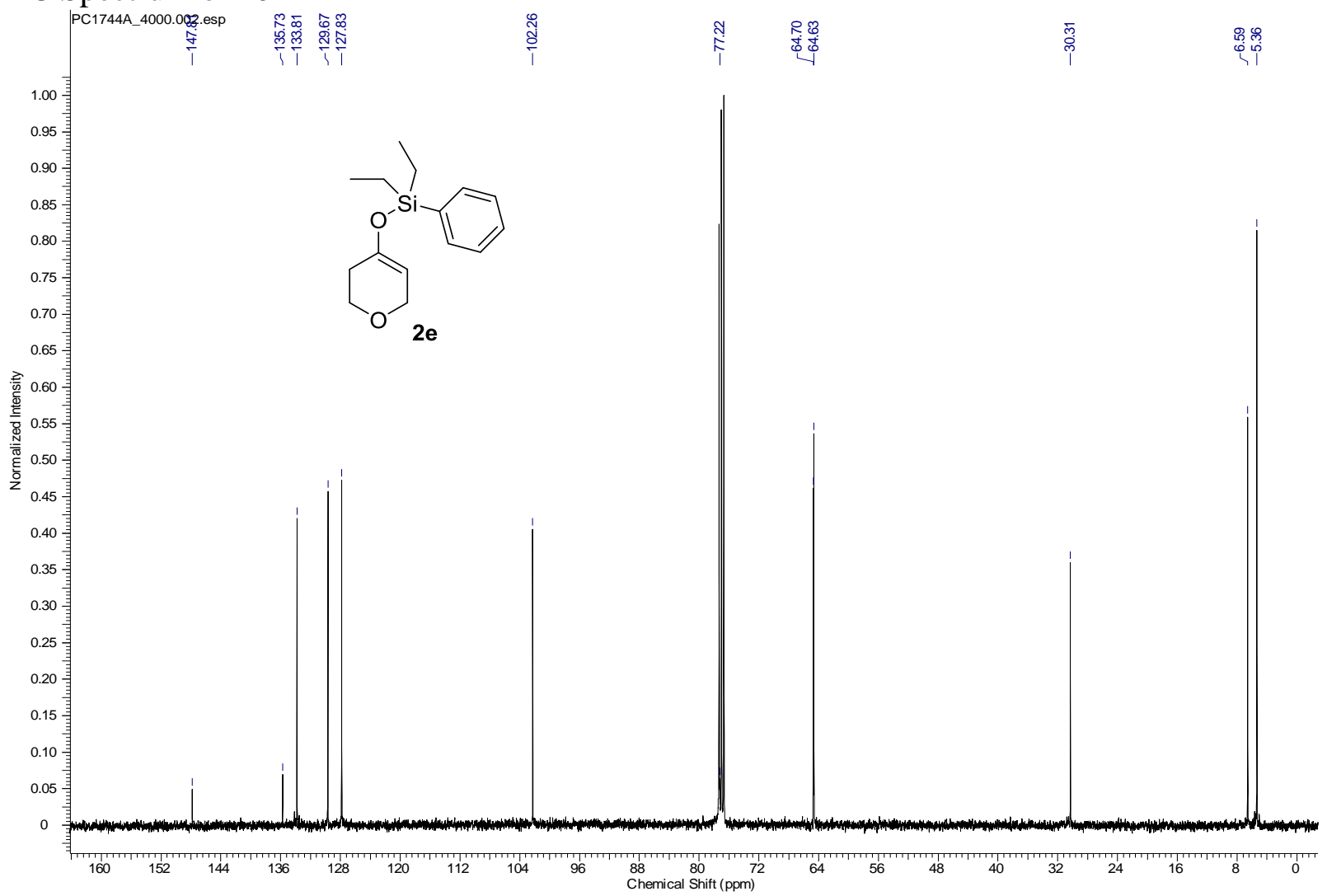




\section{${ }^{1} \mathrm{H}$ Spectrum of $\mathbf{2 f}$}

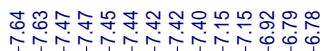

$\underset{f}{i}$

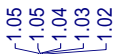<smiles>[Z17]C=C(O[Si](CC)(CC)c1ccccc1)c1ccccc1OC</smiles>
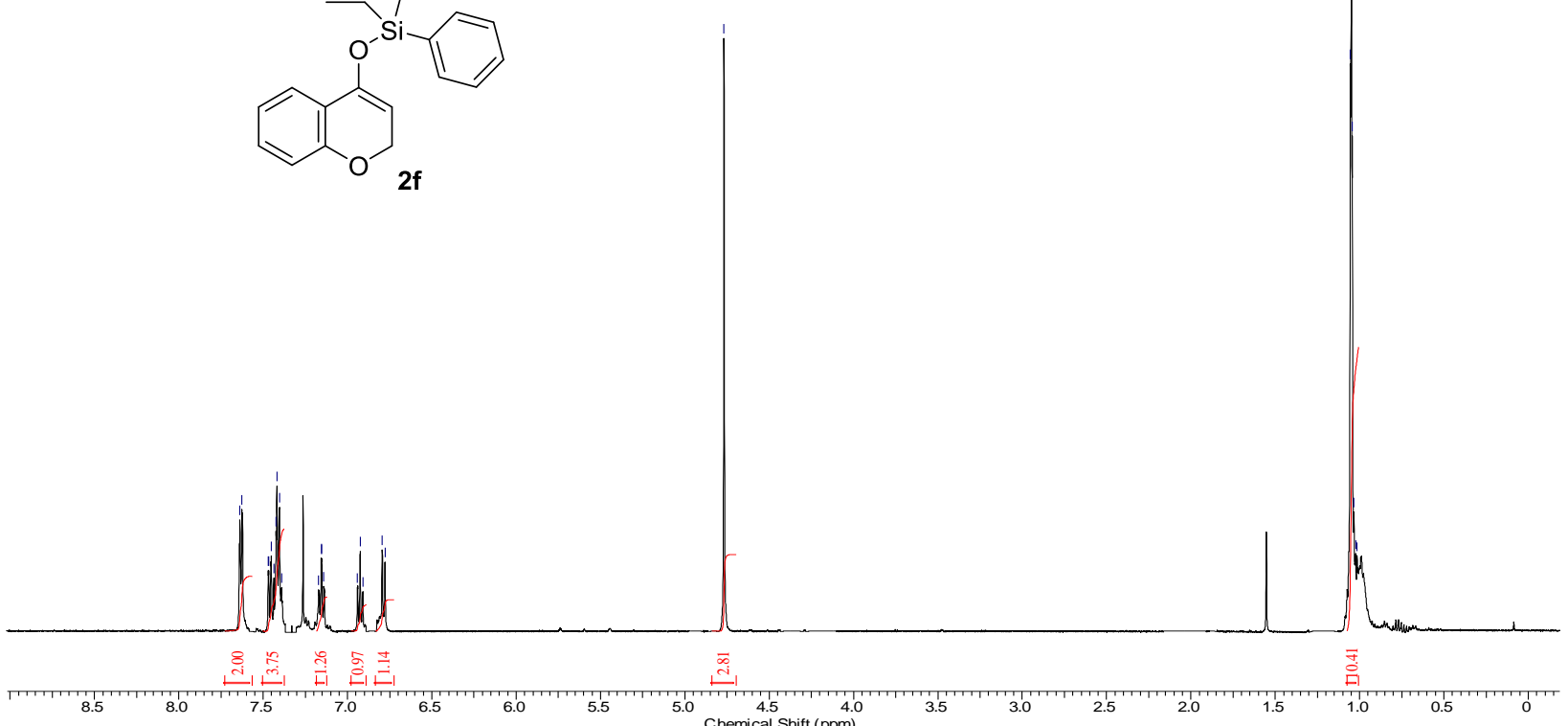

${ }^{13} \mathrm{C}$ Spectrum of $\mathbf{2 f}$

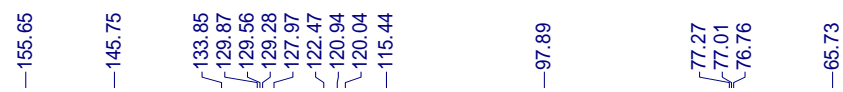

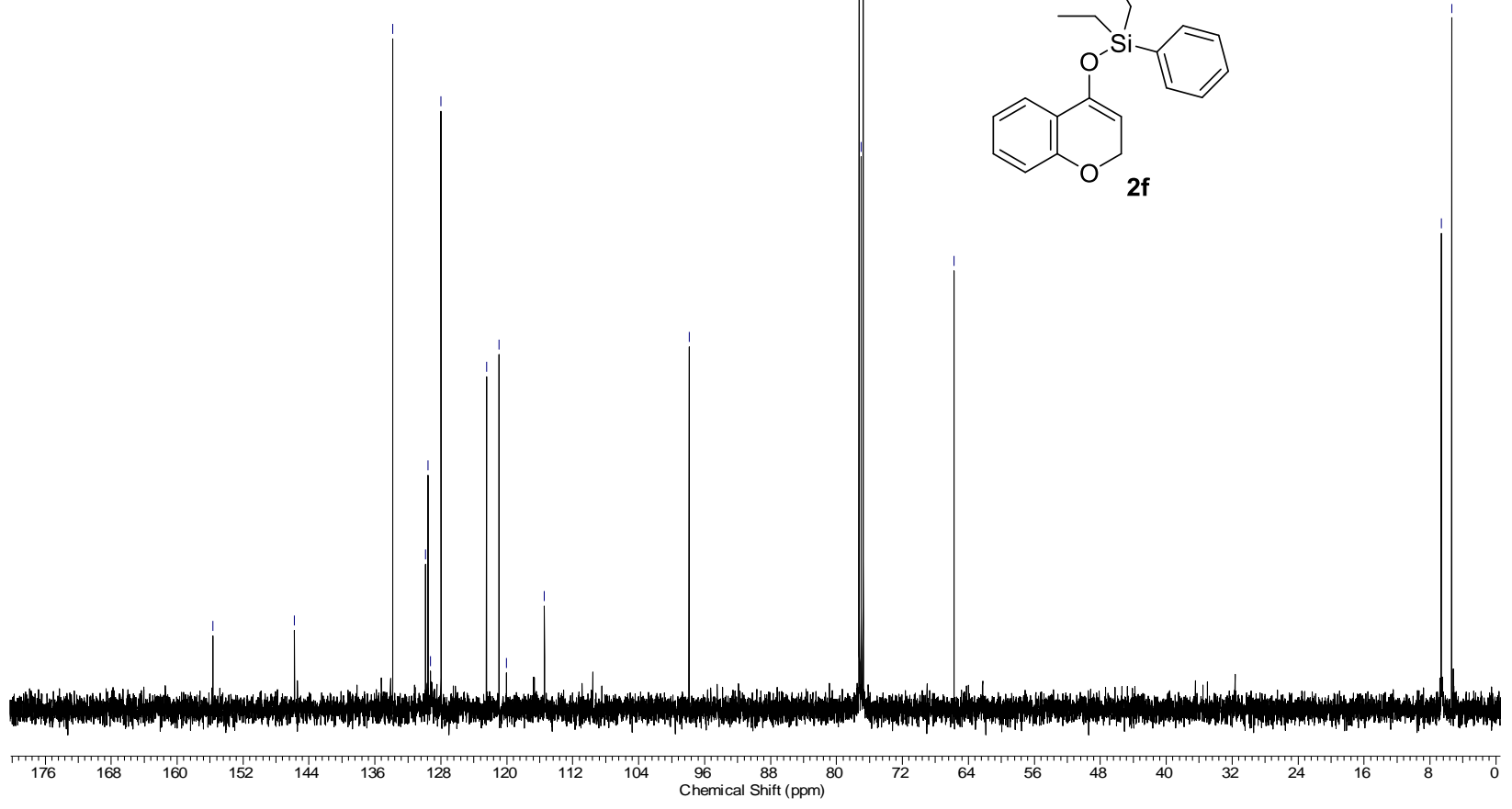




\section{${ }^{1} \mathrm{H}$ Spectrum of $\mathbf{2 g}$}

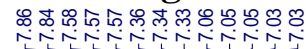

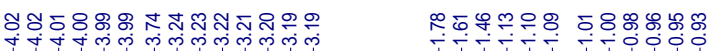
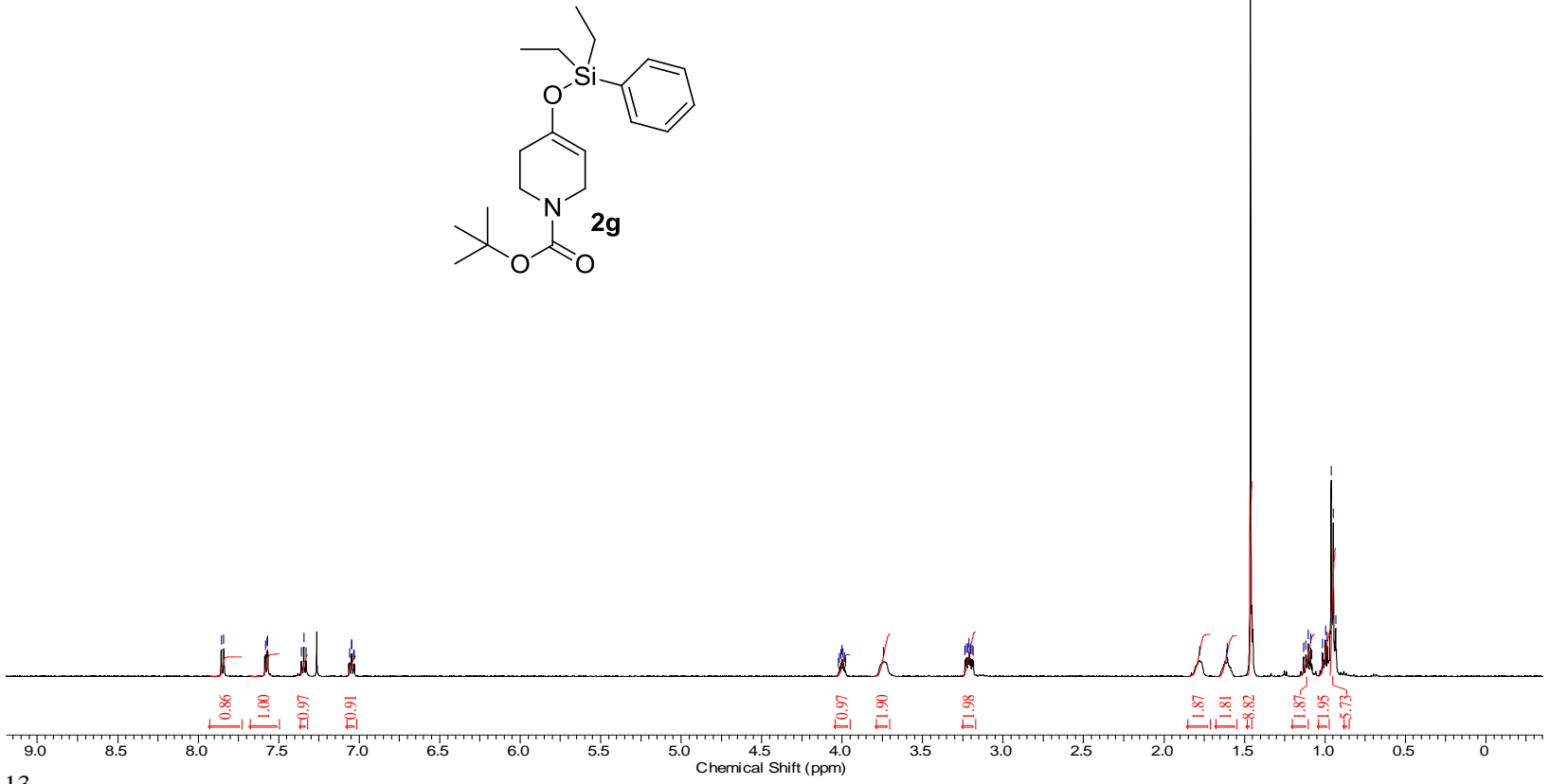

\section{${ }^{13} \mathrm{C}$ Spectrum of $\mathbf{2 g}$}

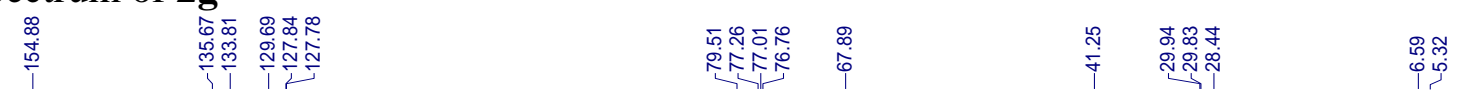

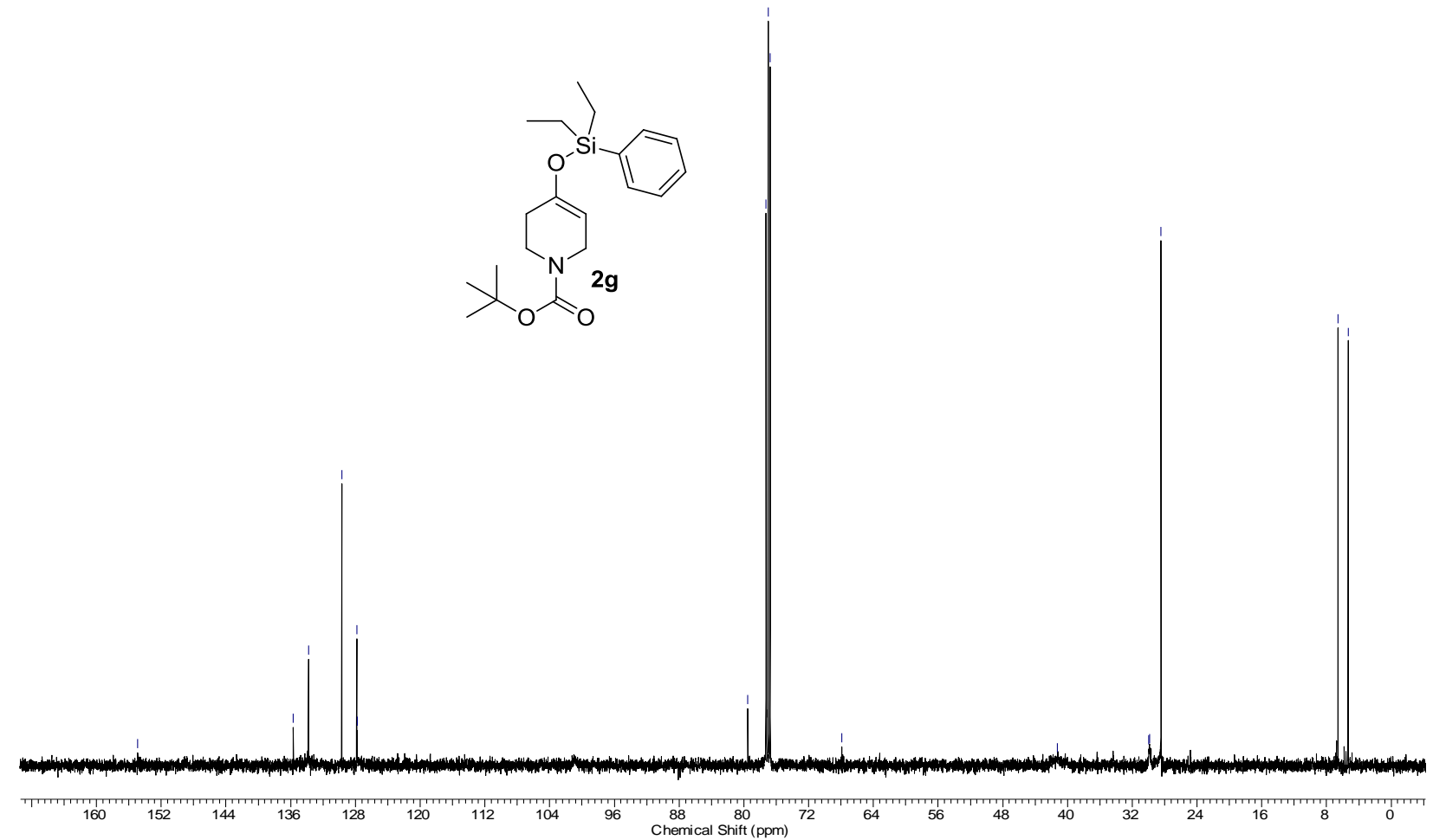




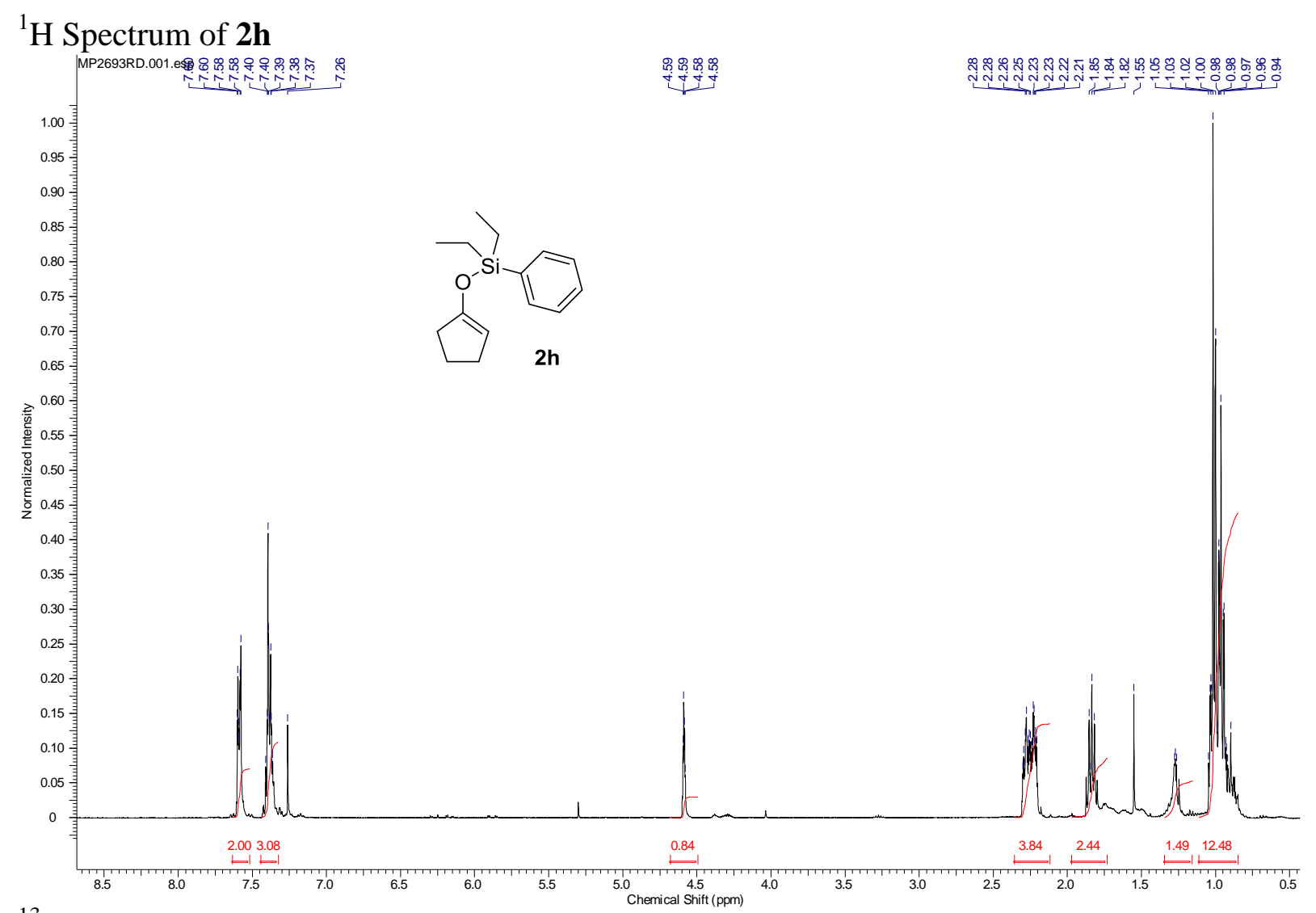

${ }^{13} \mathrm{C}$ Spectrum of $\mathbf{2 h}$

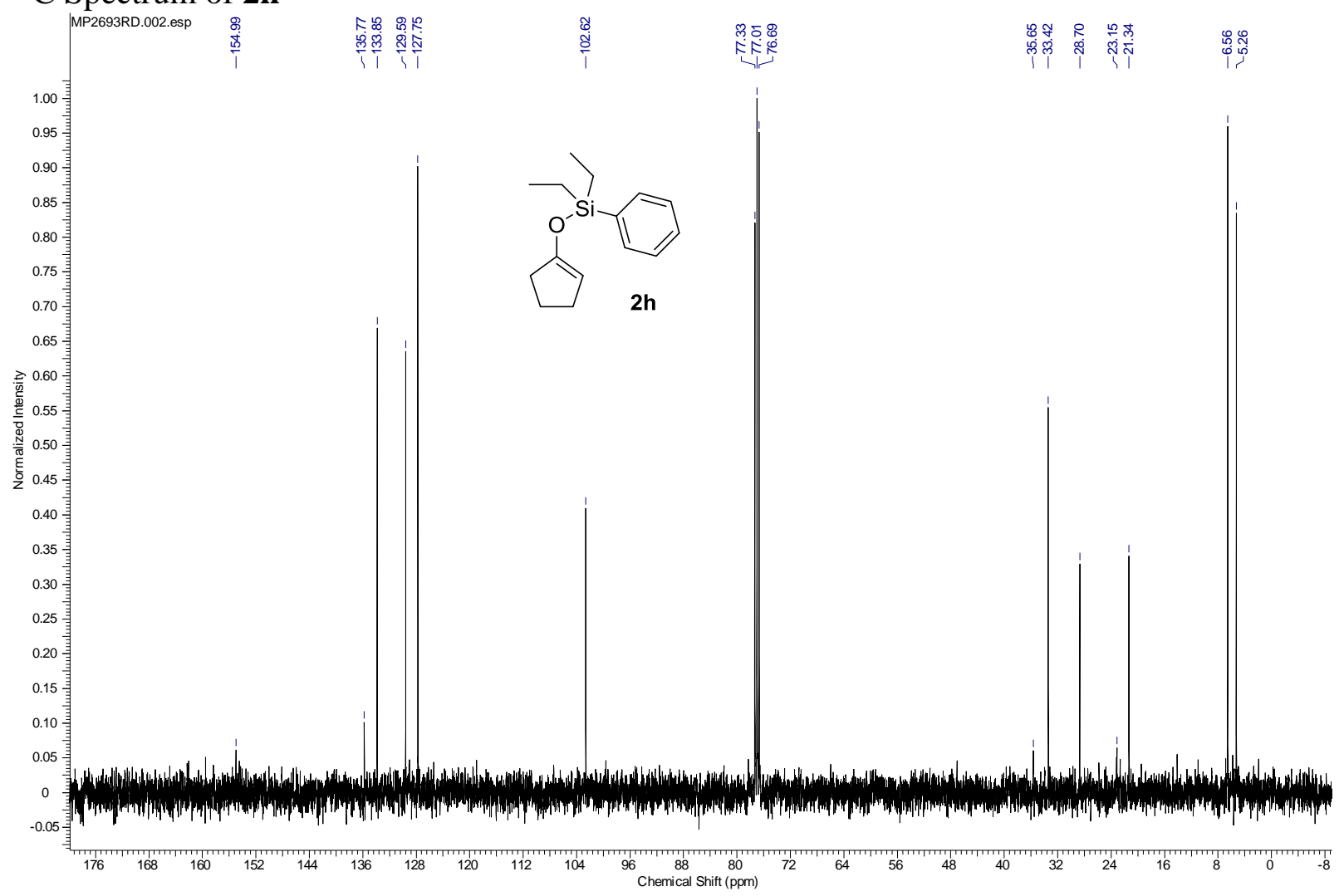




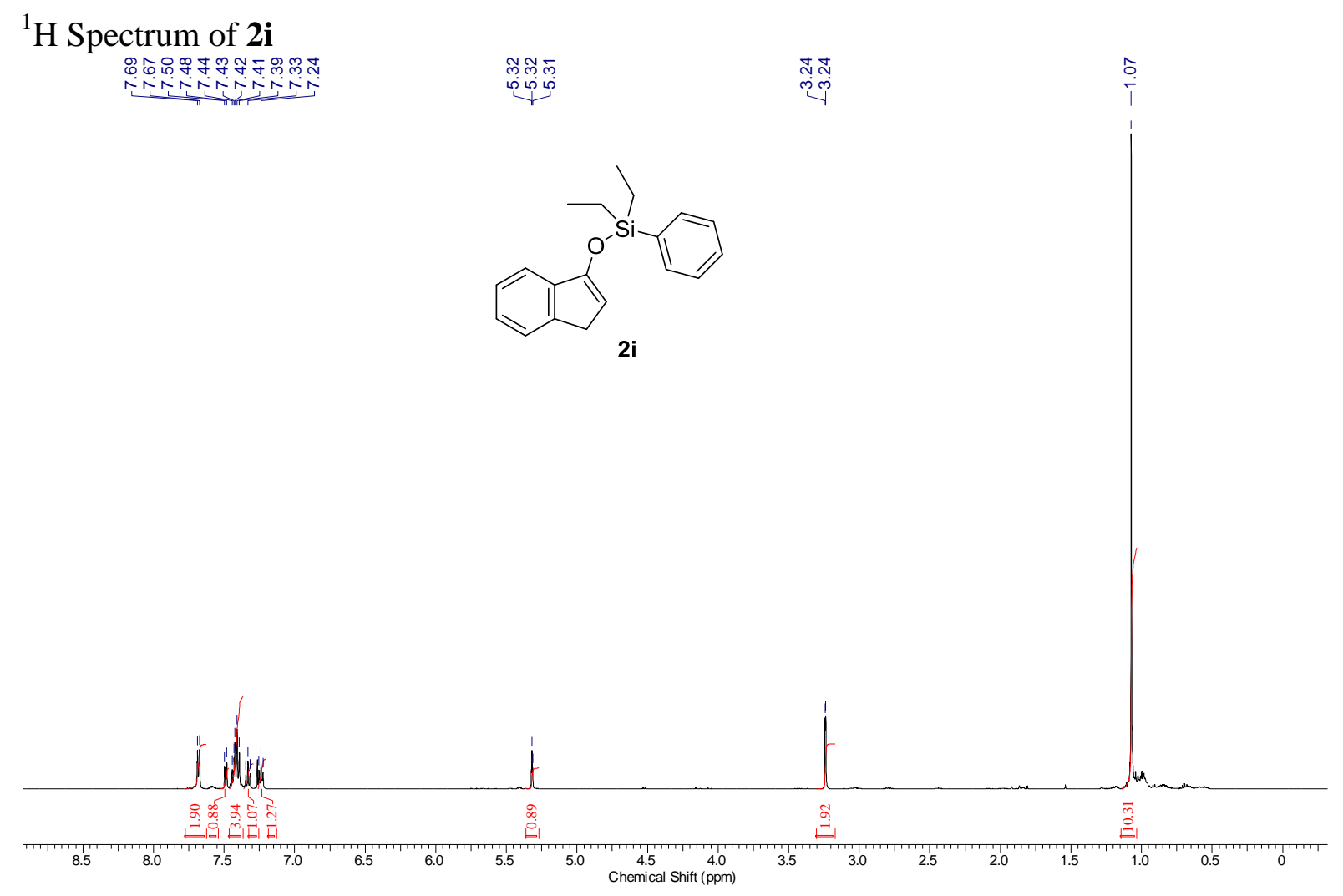

${ }^{13} \mathrm{C}$ Spectrum of $\mathbf{2 i}$

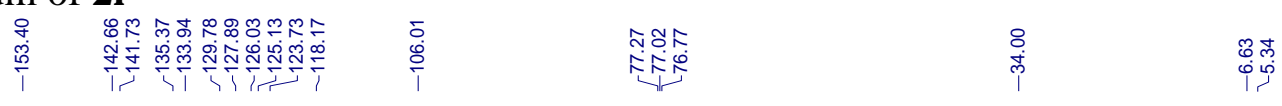

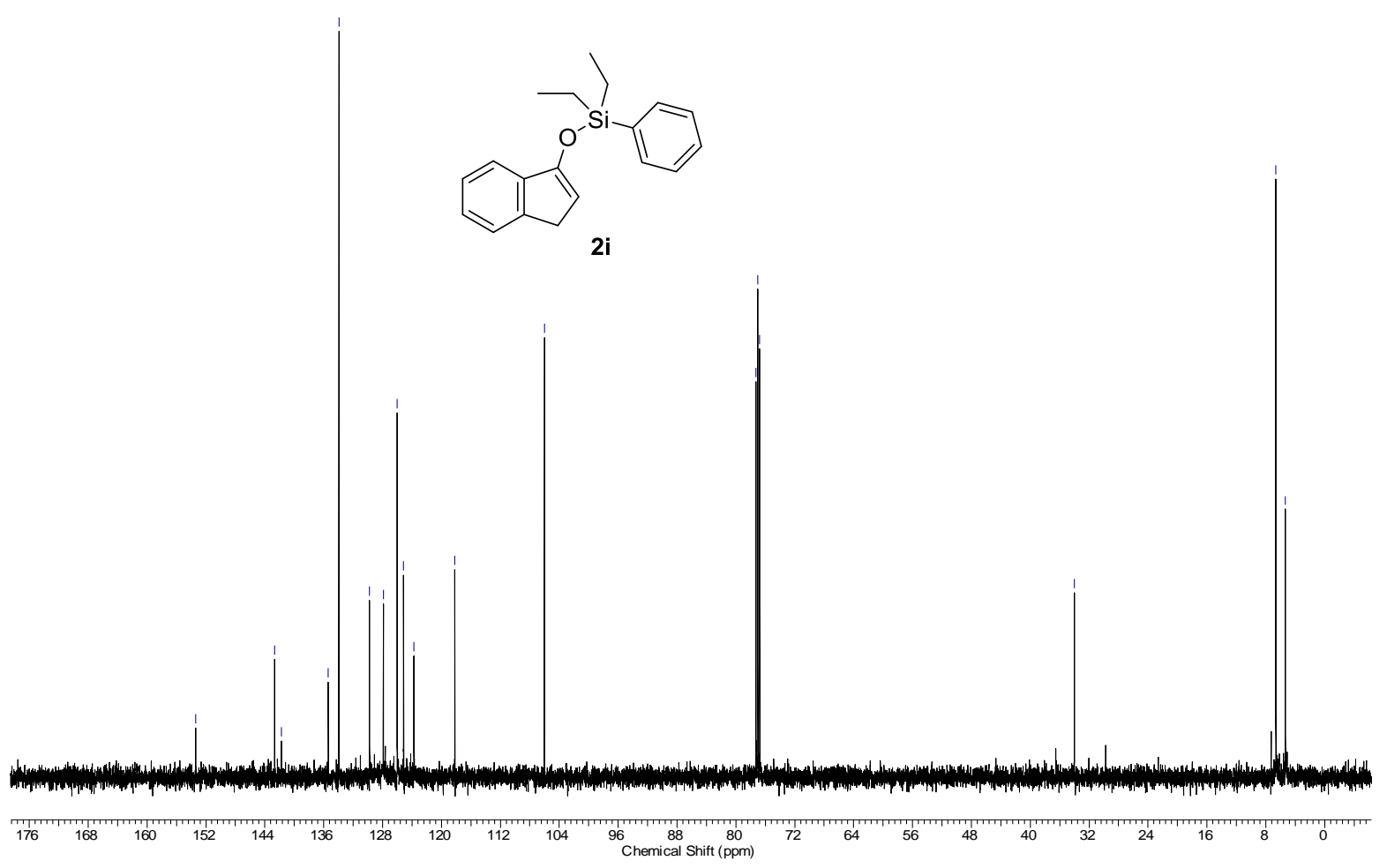




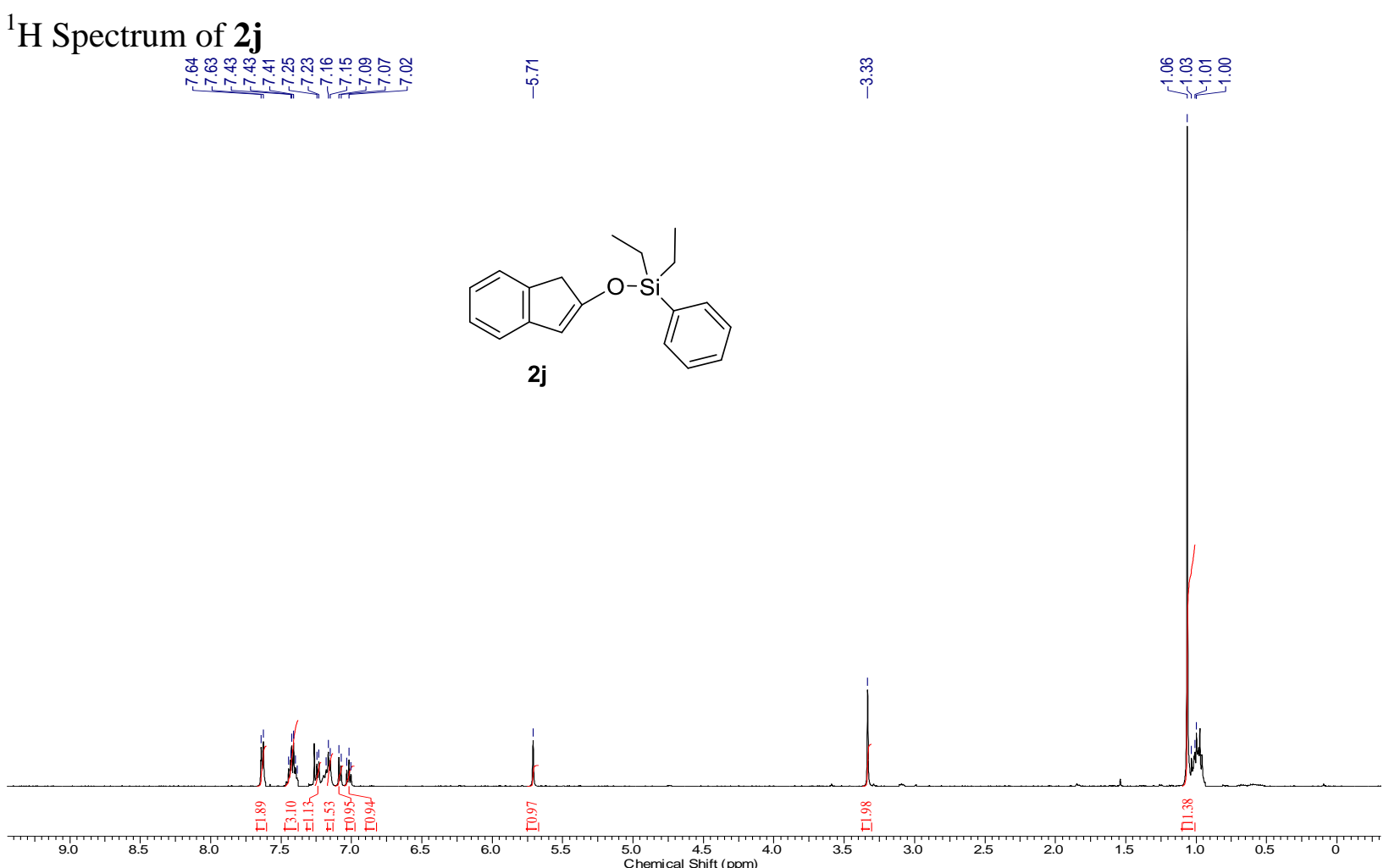

${ }^{13} \mathrm{C}$ Spectrum of $\mathbf{2 j}$
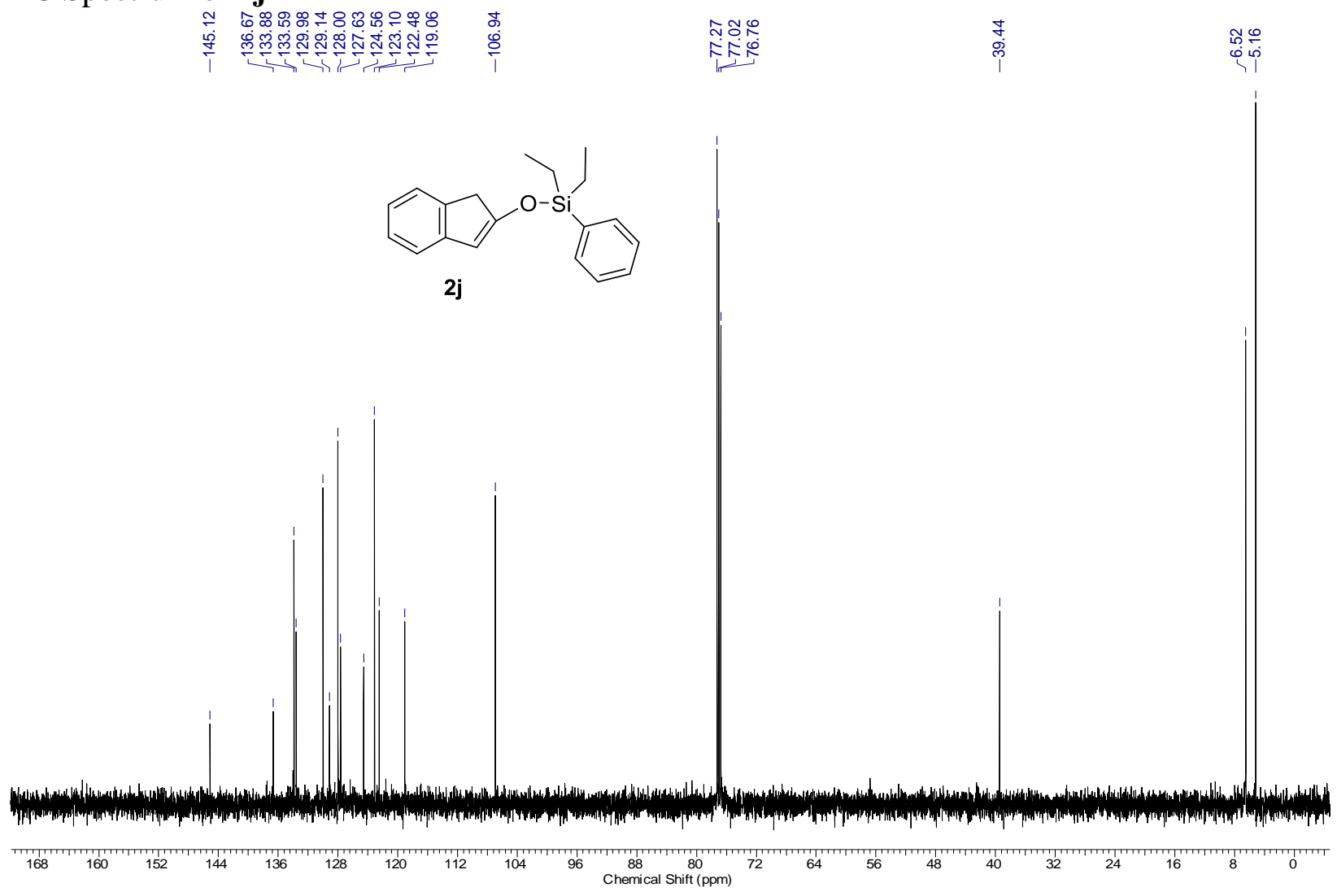


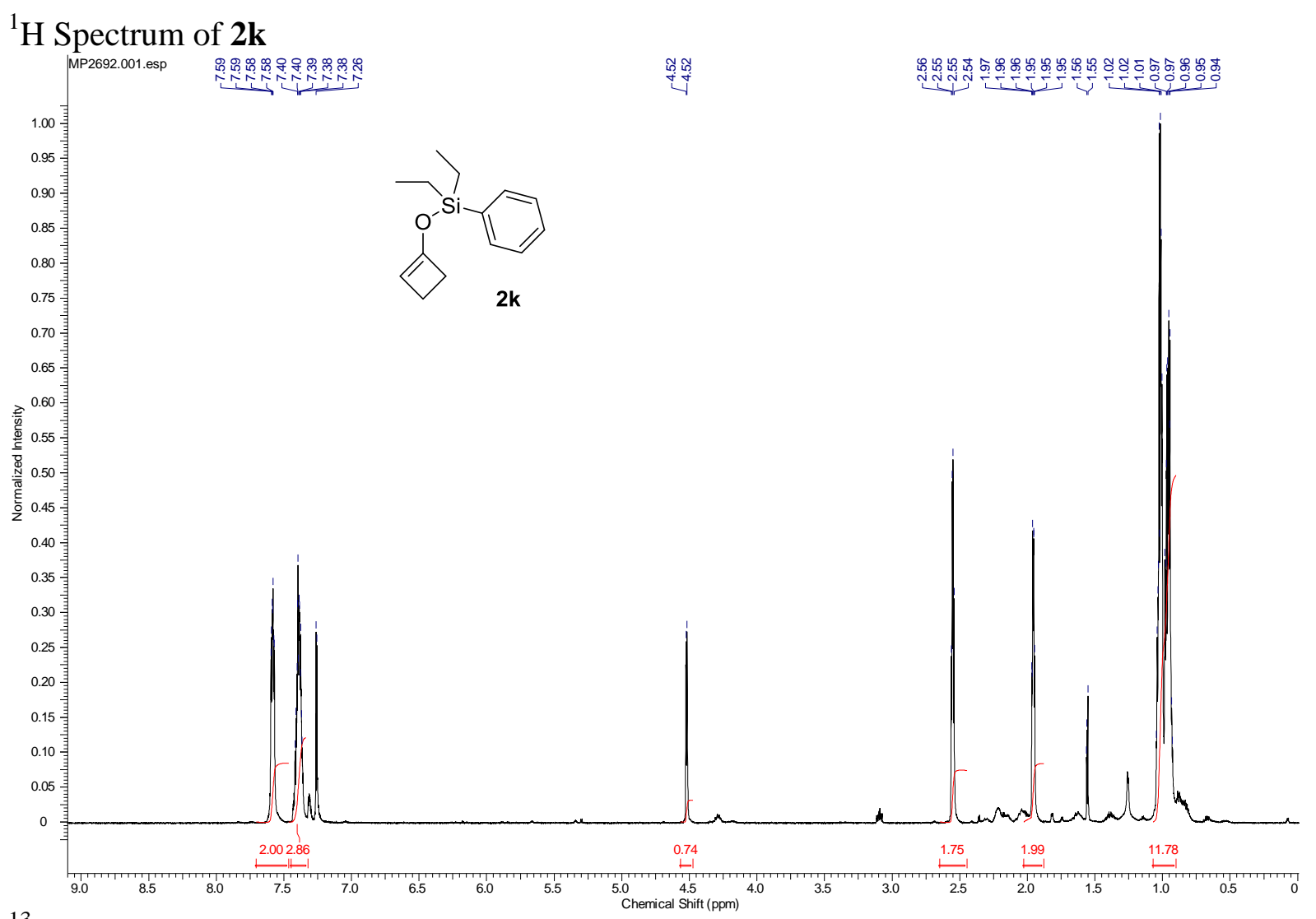

${ }^{13} \mathrm{C}$ Spectrum of $\mathbf{2 k}$

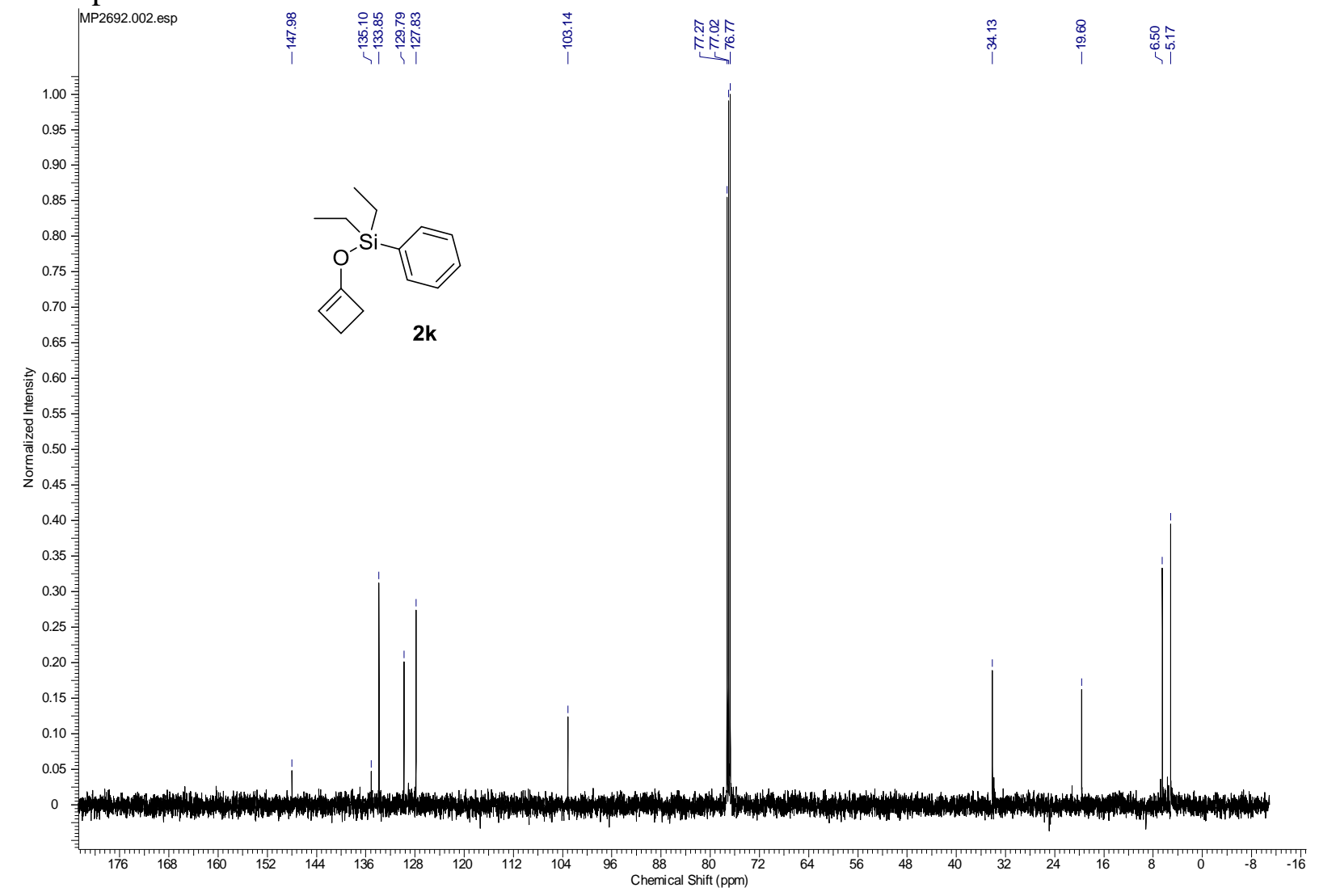




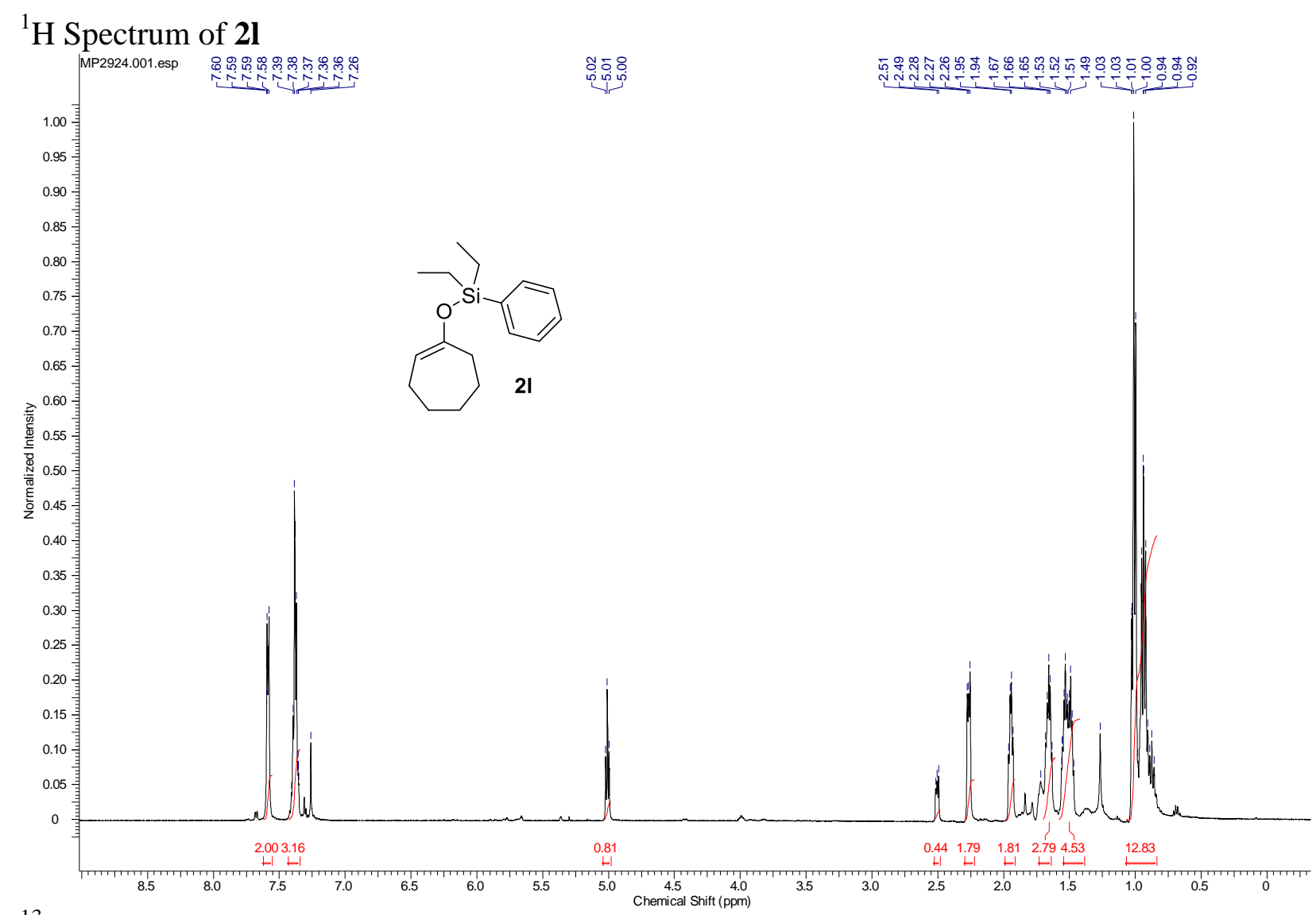

\section{${ }^{13} \mathrm{C}$ Spectrum of $\mathbf{2 l}$}

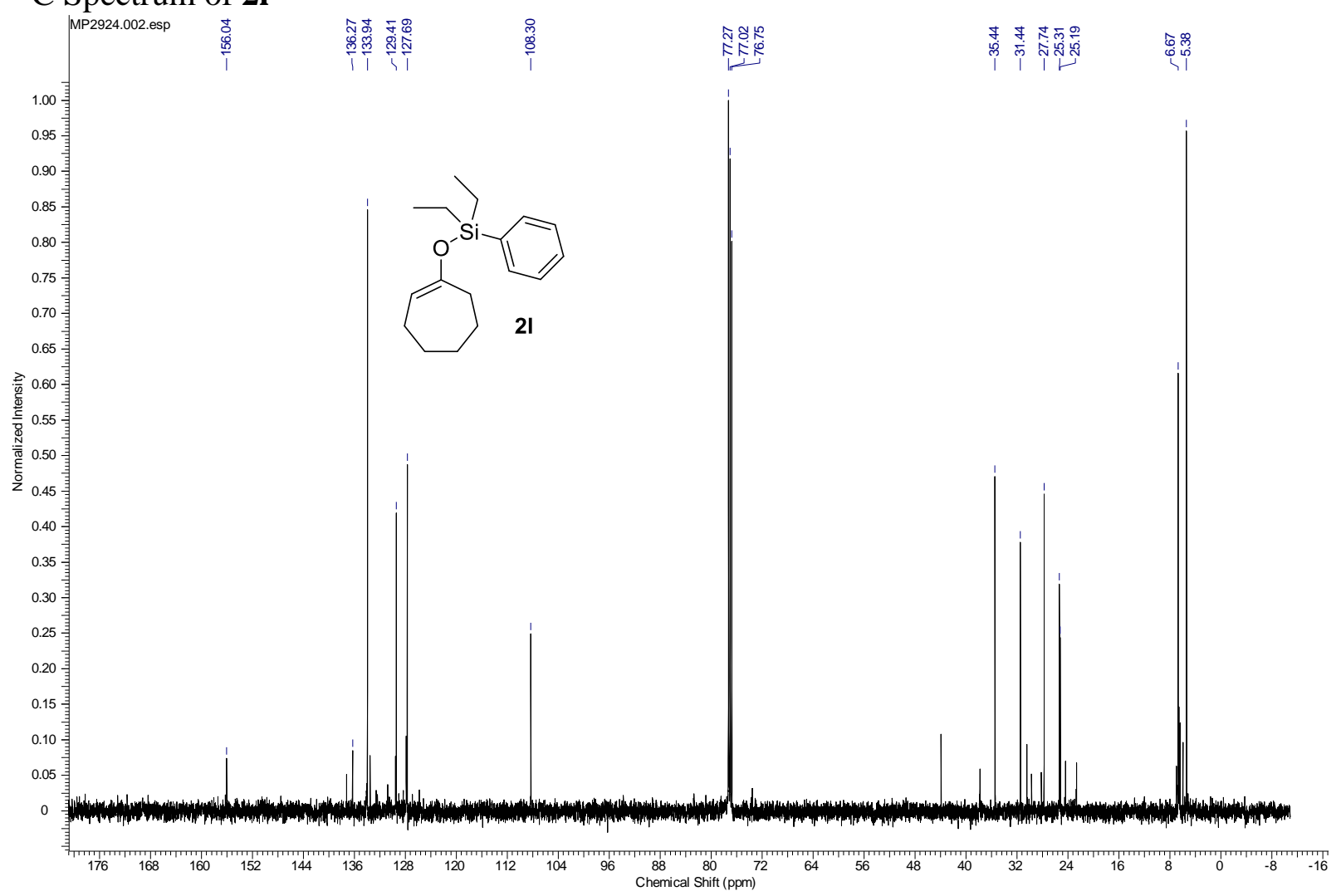




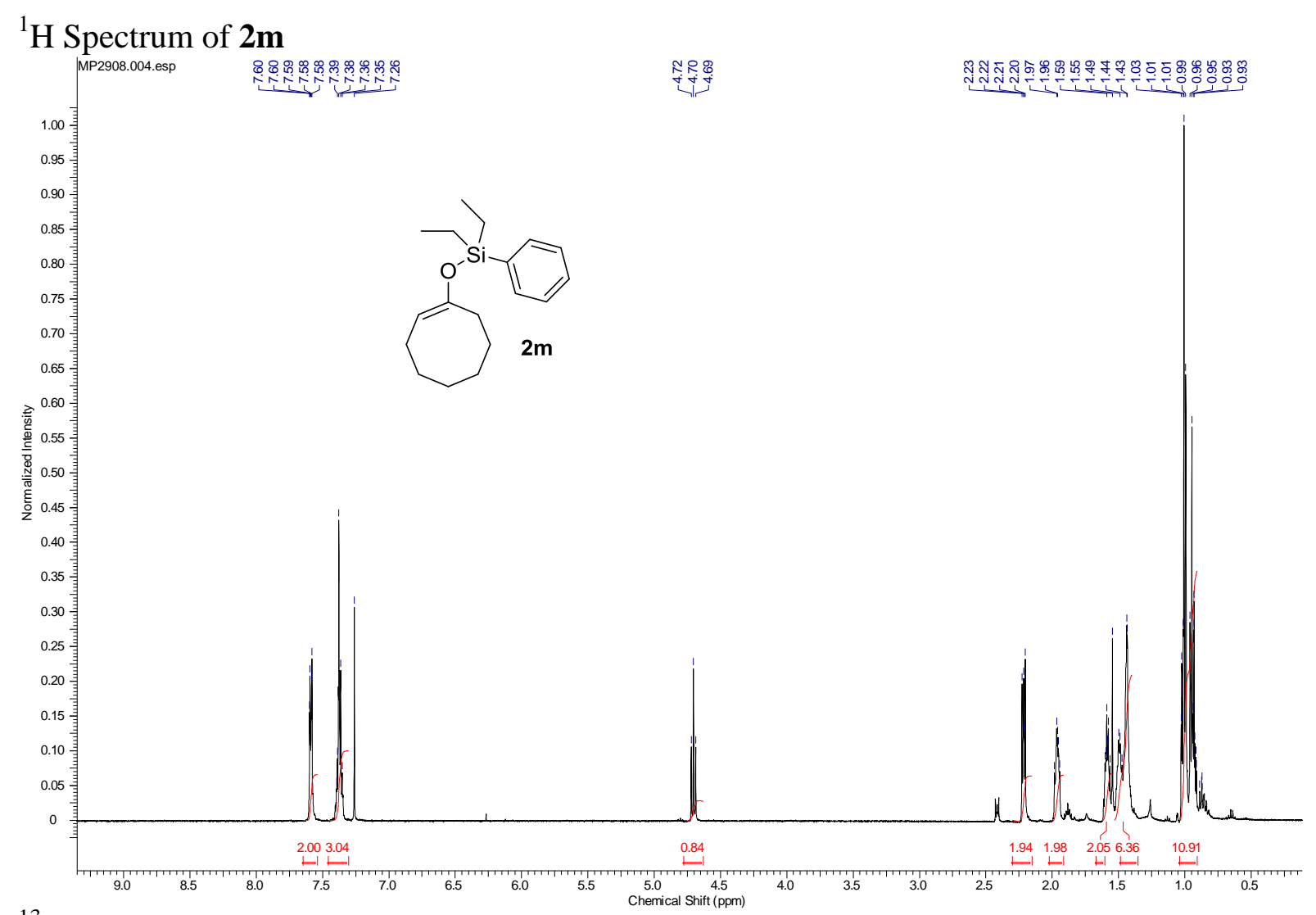

${ }^{13} \mathrm{C}$ Spectrum of $\mathbf{2 m}$

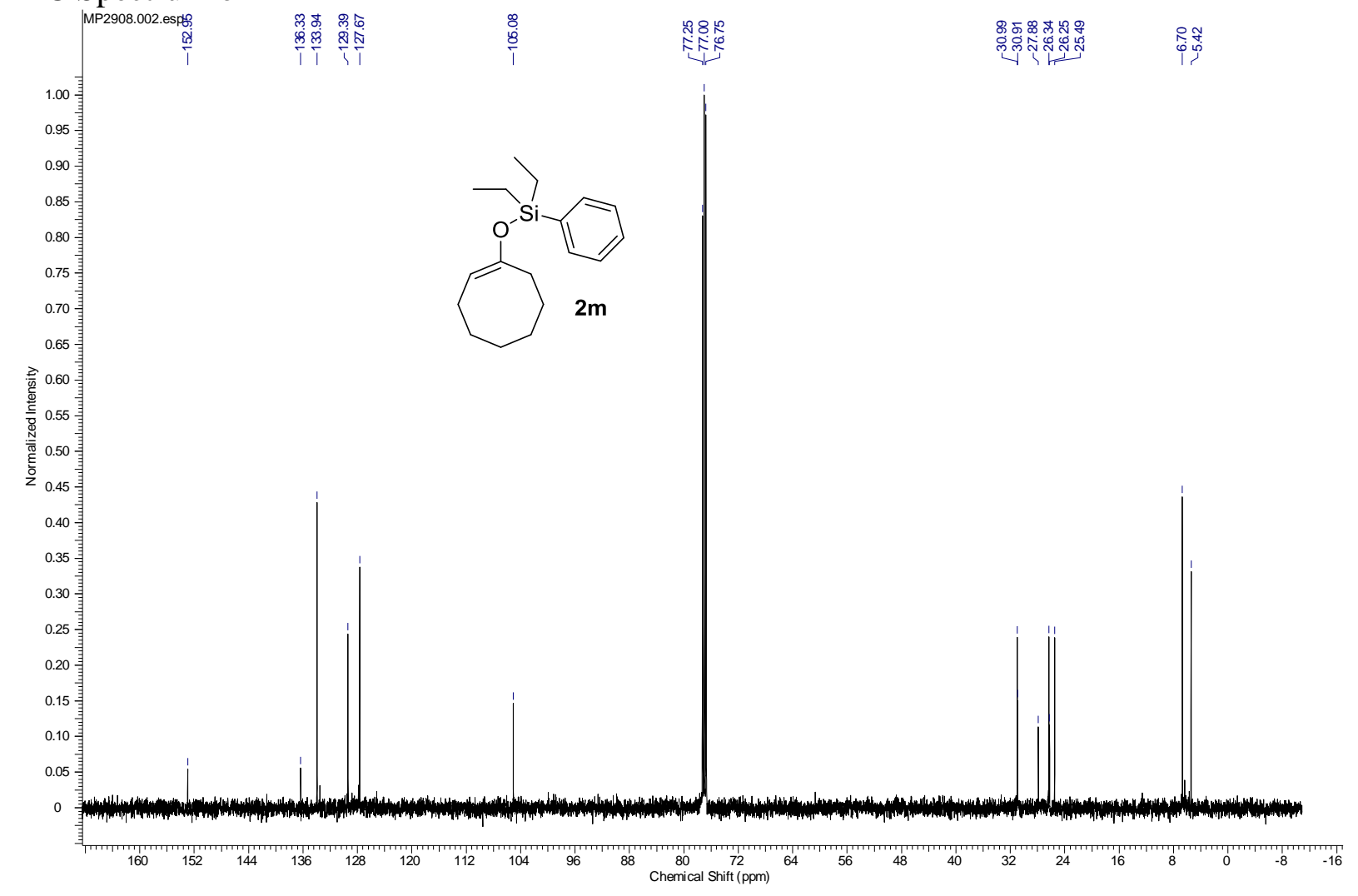




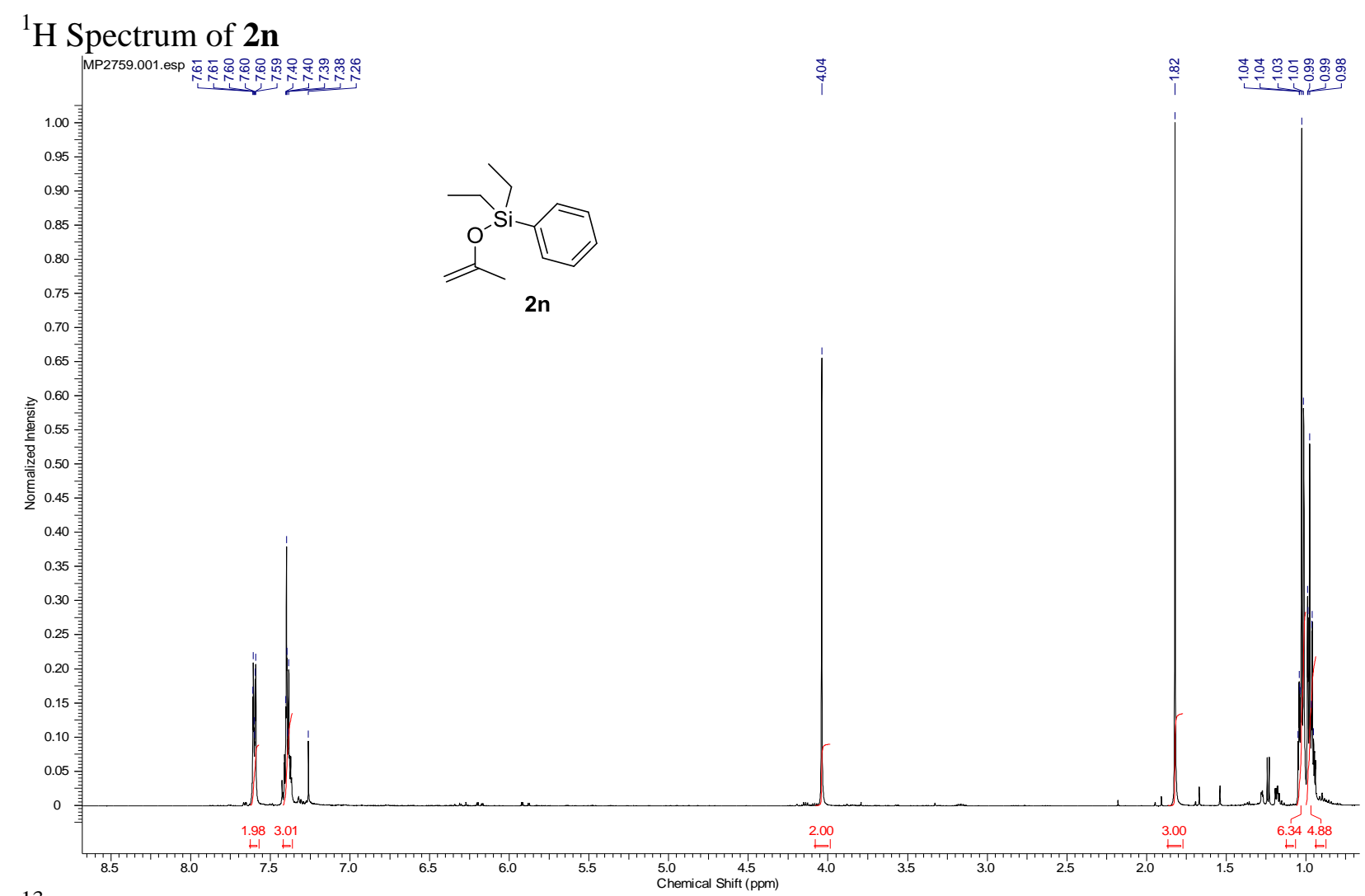

${ }^{13} \mathrm{C}$ Spectrum of $\mathbf{2 n}$

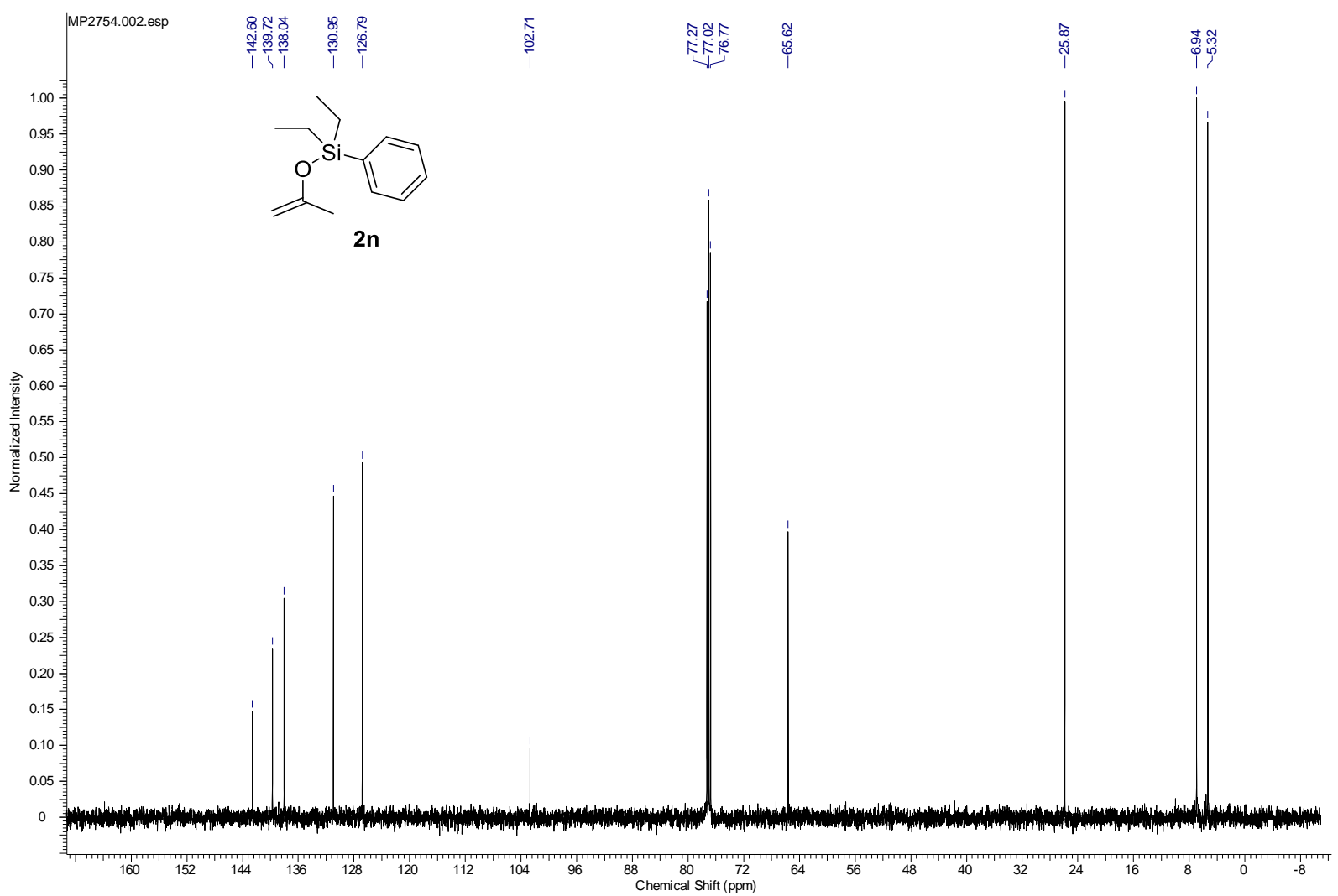




\section{${ }^{1} \mathrm{H}$ Spectrum of $\mathbf{2 0}$}

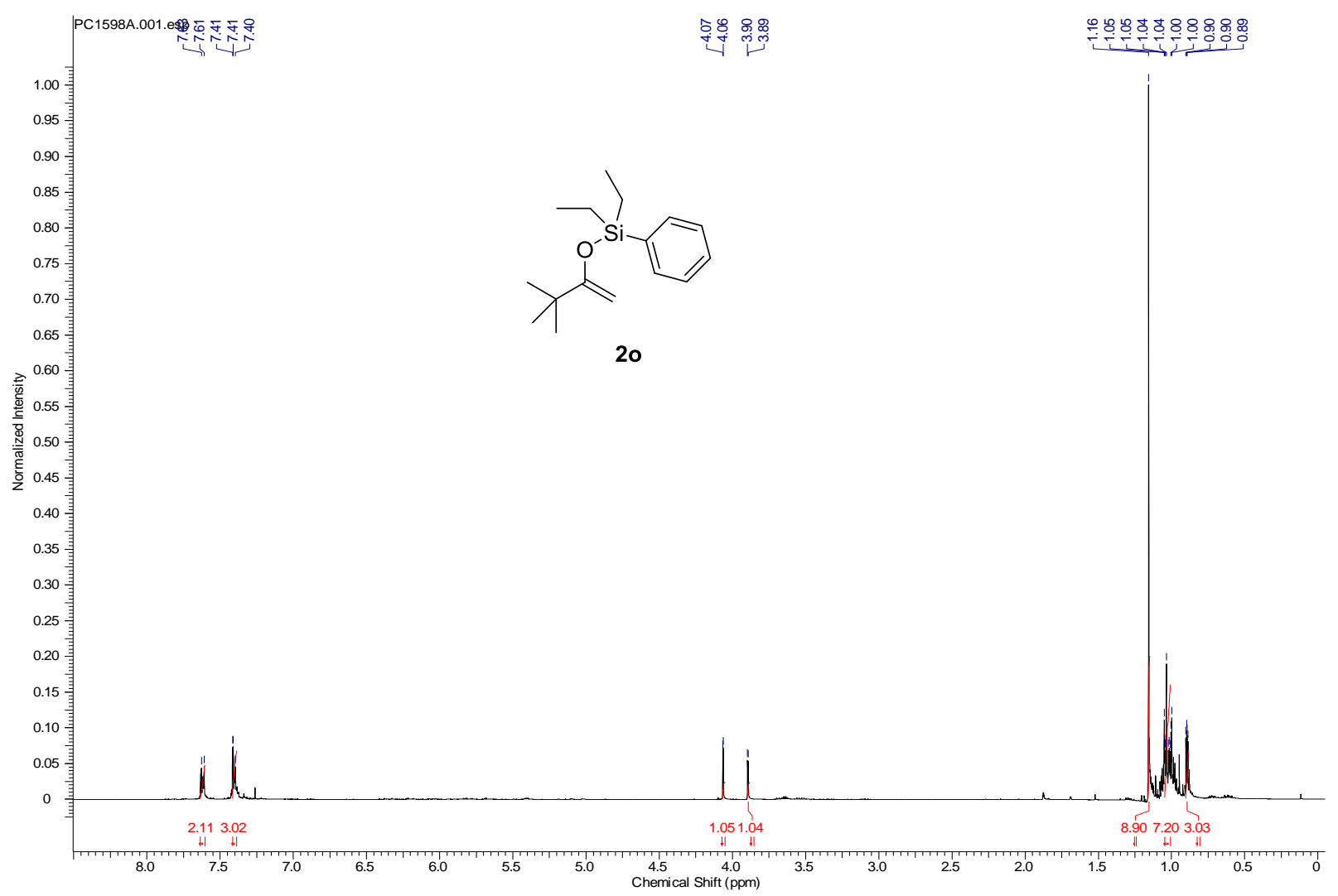

\section{${ }^{13} \mathrm{C}$ Spectrum of 20}

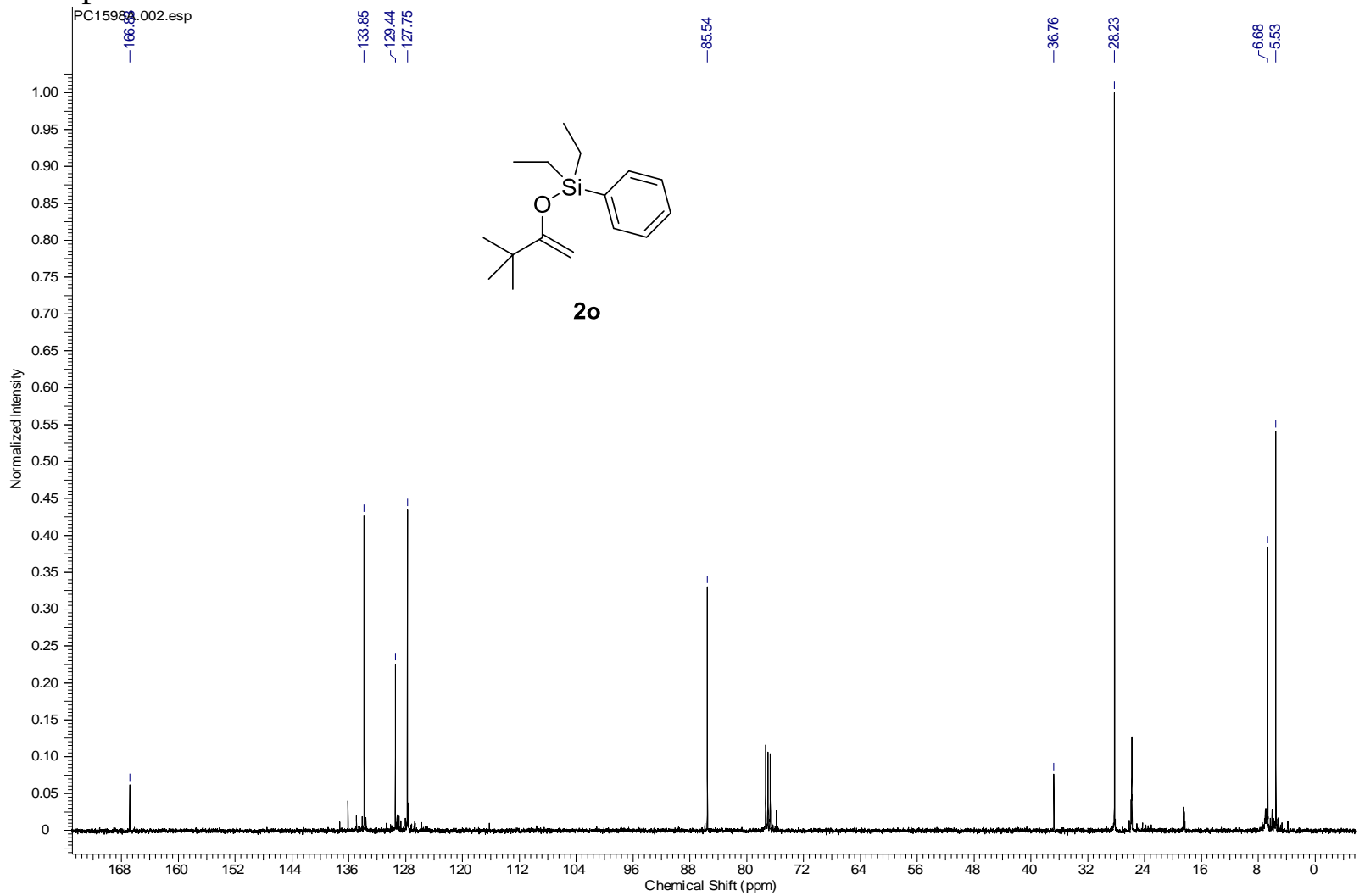




\section{${ }^{1} \mathrm{H}$ Spectrum of $\mathbf{2 p}$}

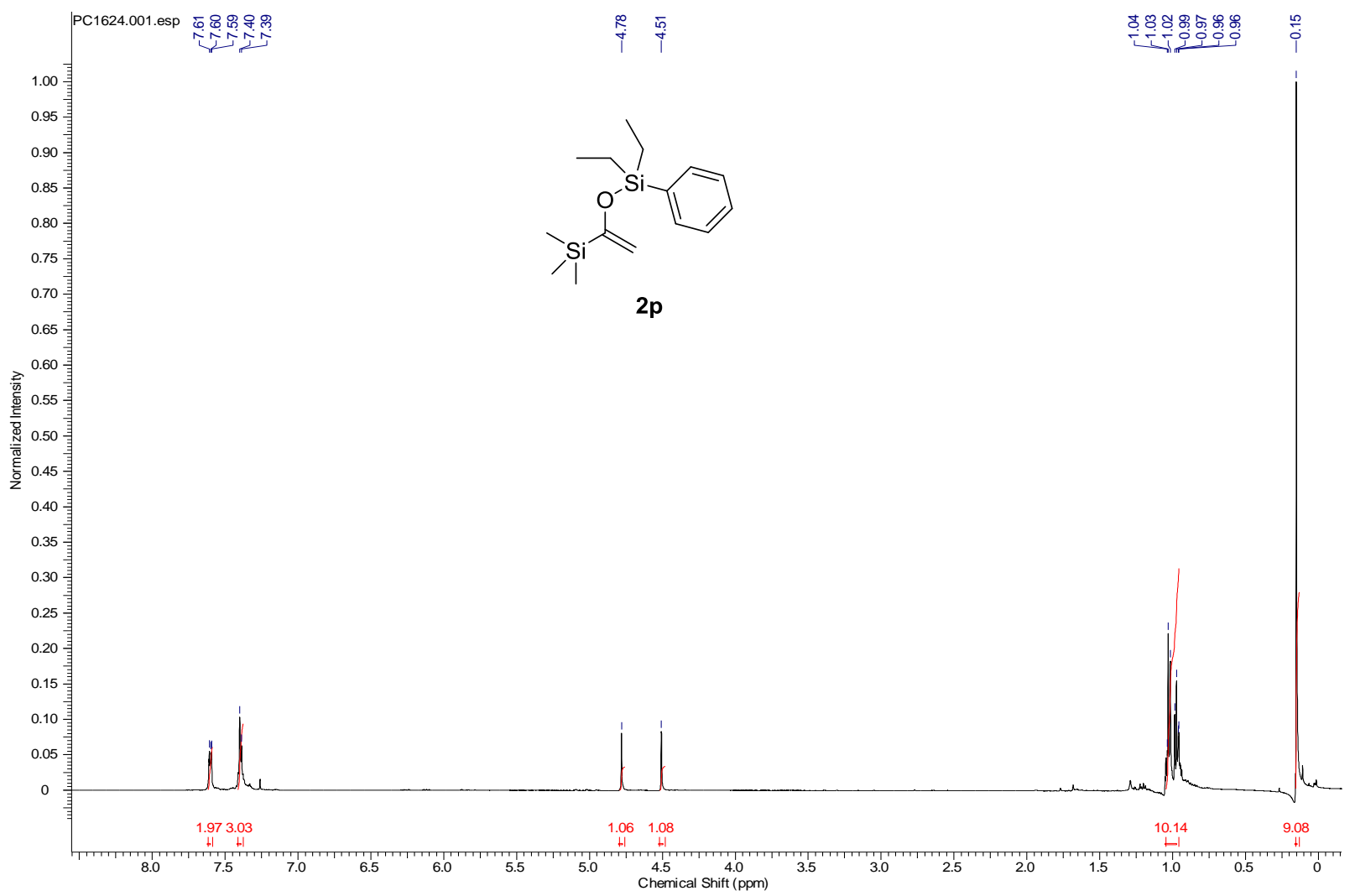

\section{${ }^{13} \mathrm{C}$ Spectrum of $\mathbf{2 p}$}

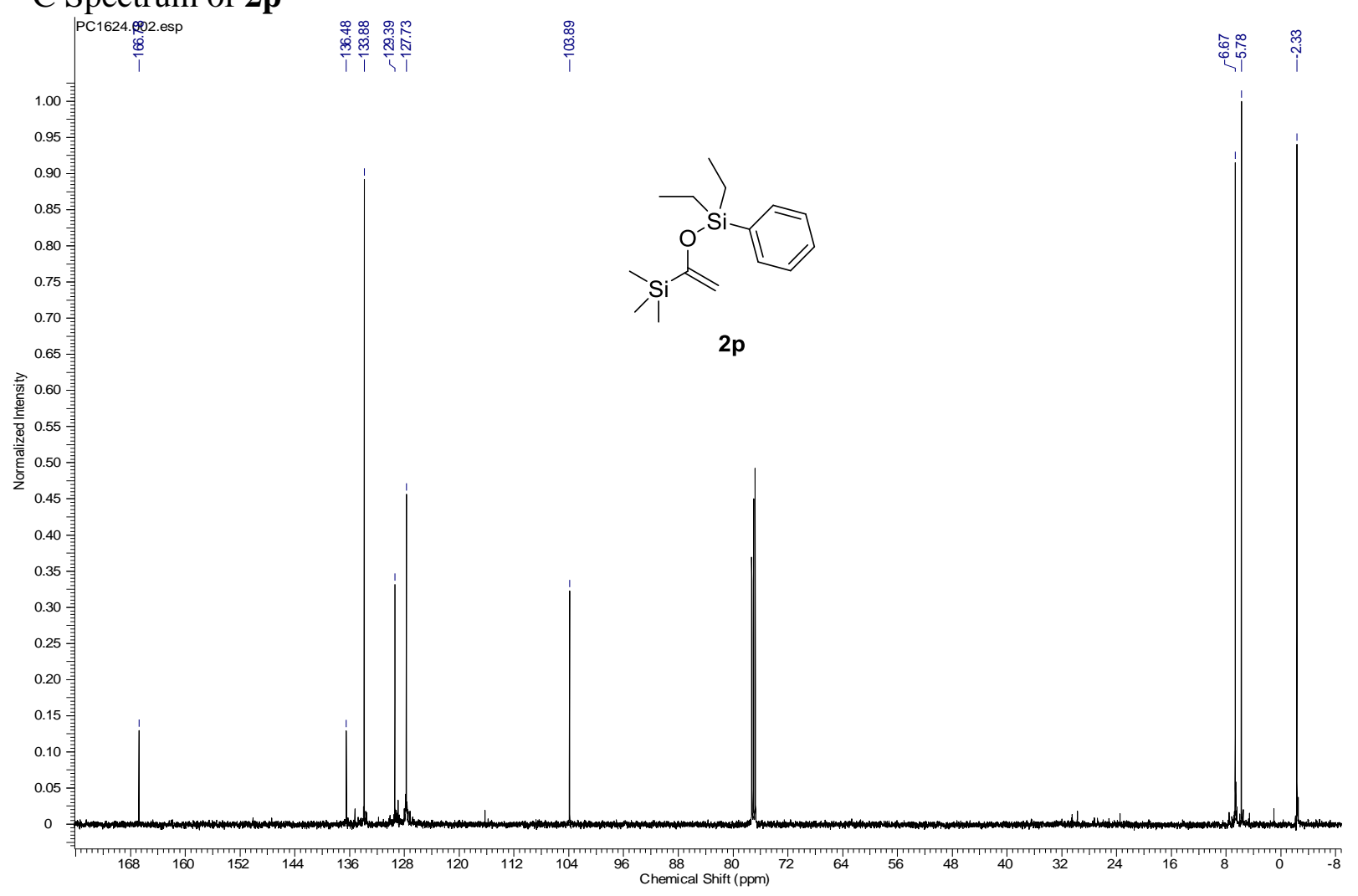




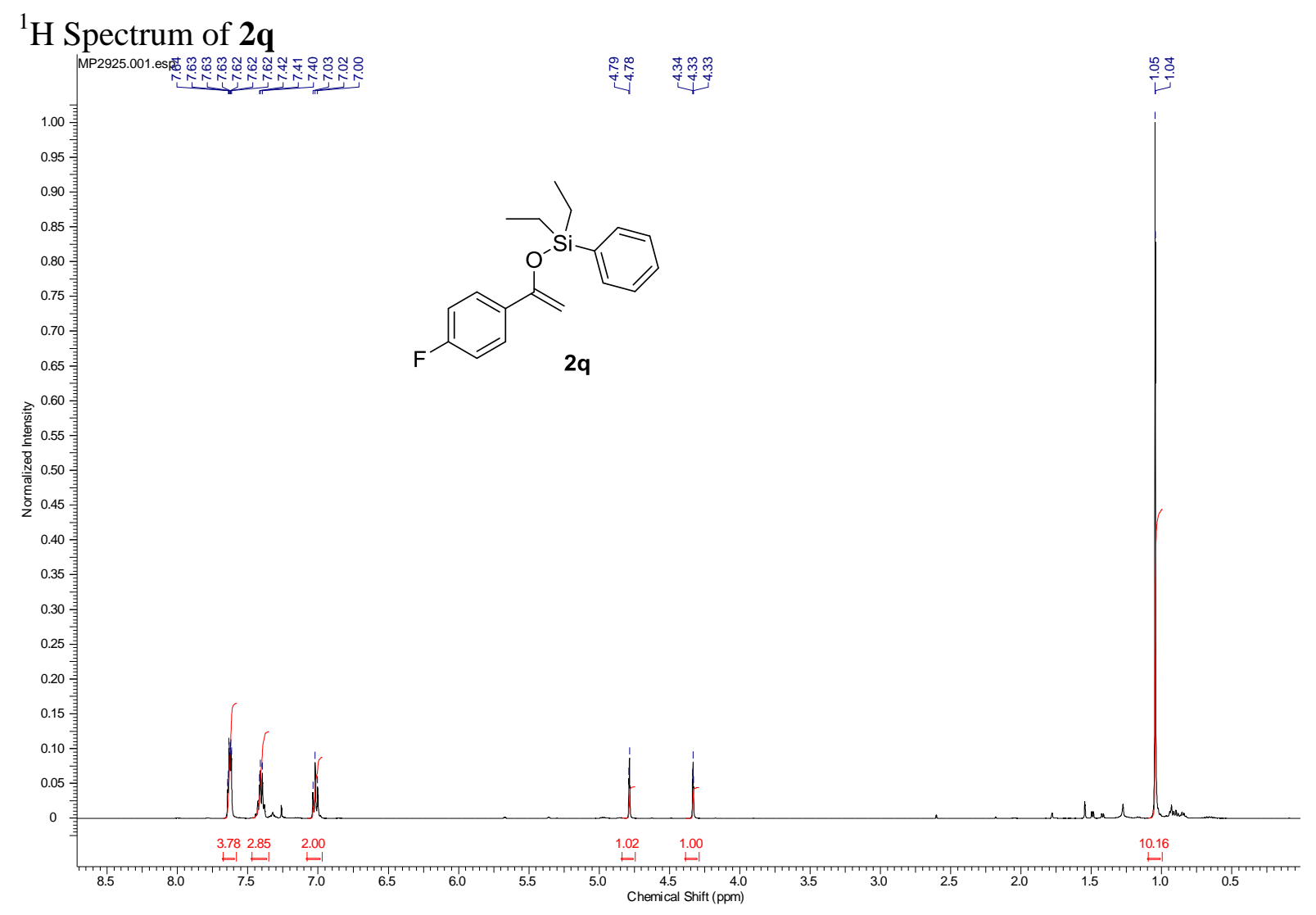

\section{${ }^{13} \mathrm{C}$ Spectrum of $\mathbf{2 q}$}

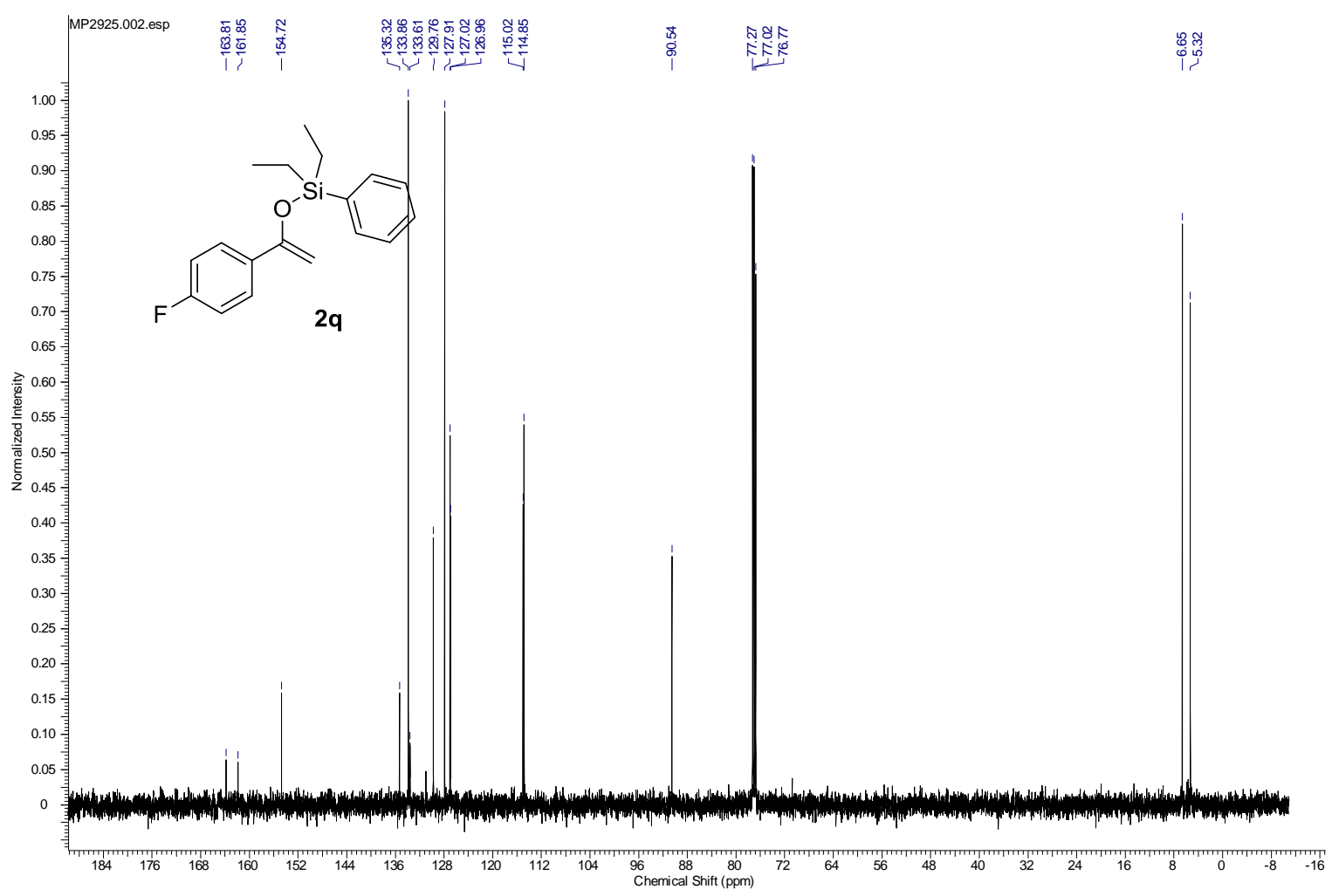




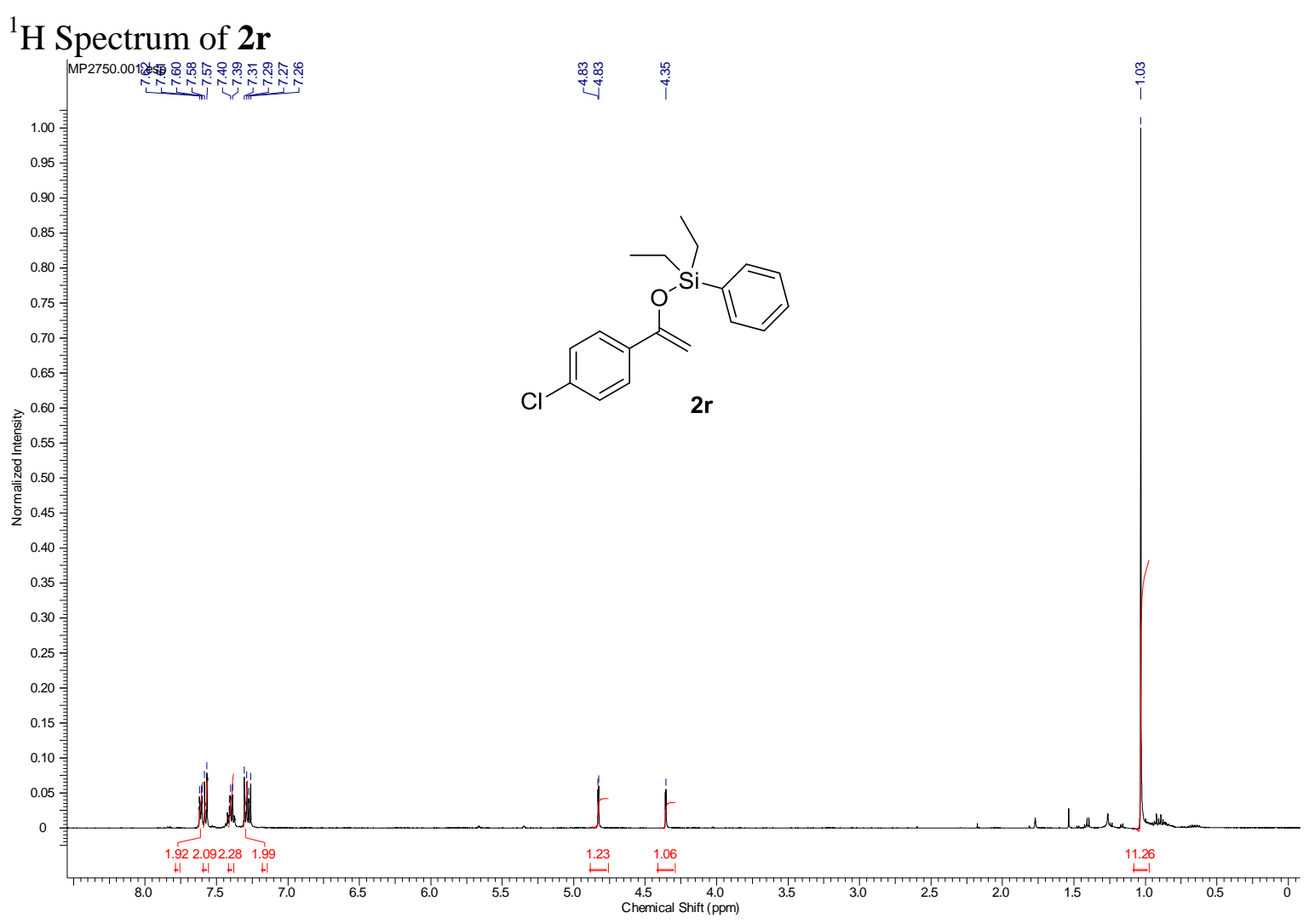

${ }^{13} \mathrm{C}$ Spectrum of $\mathbf{2 r}$

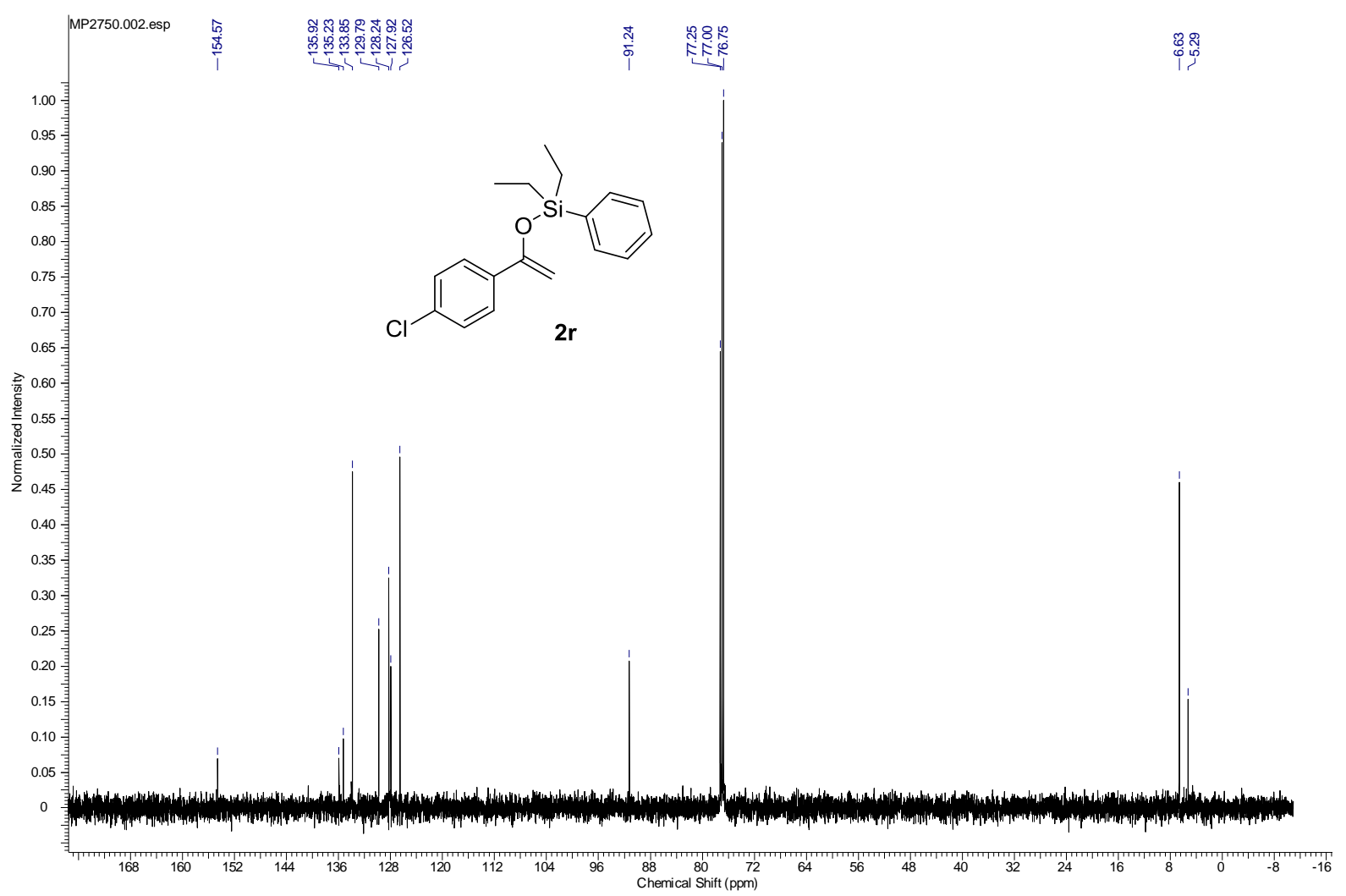




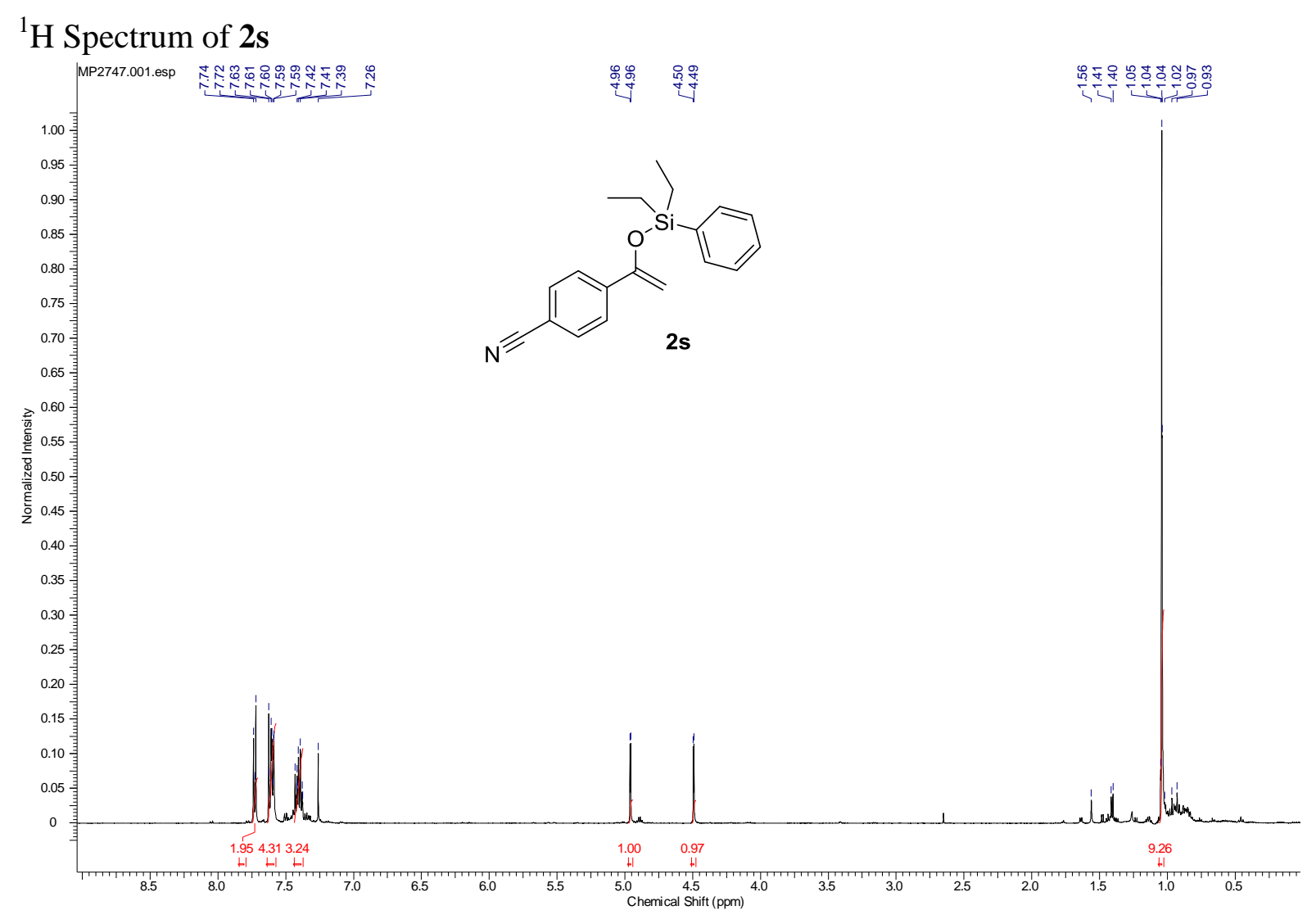

${ }^{13} \mathrm{C}$ Spectrum of $2 \mathrm{~s}$

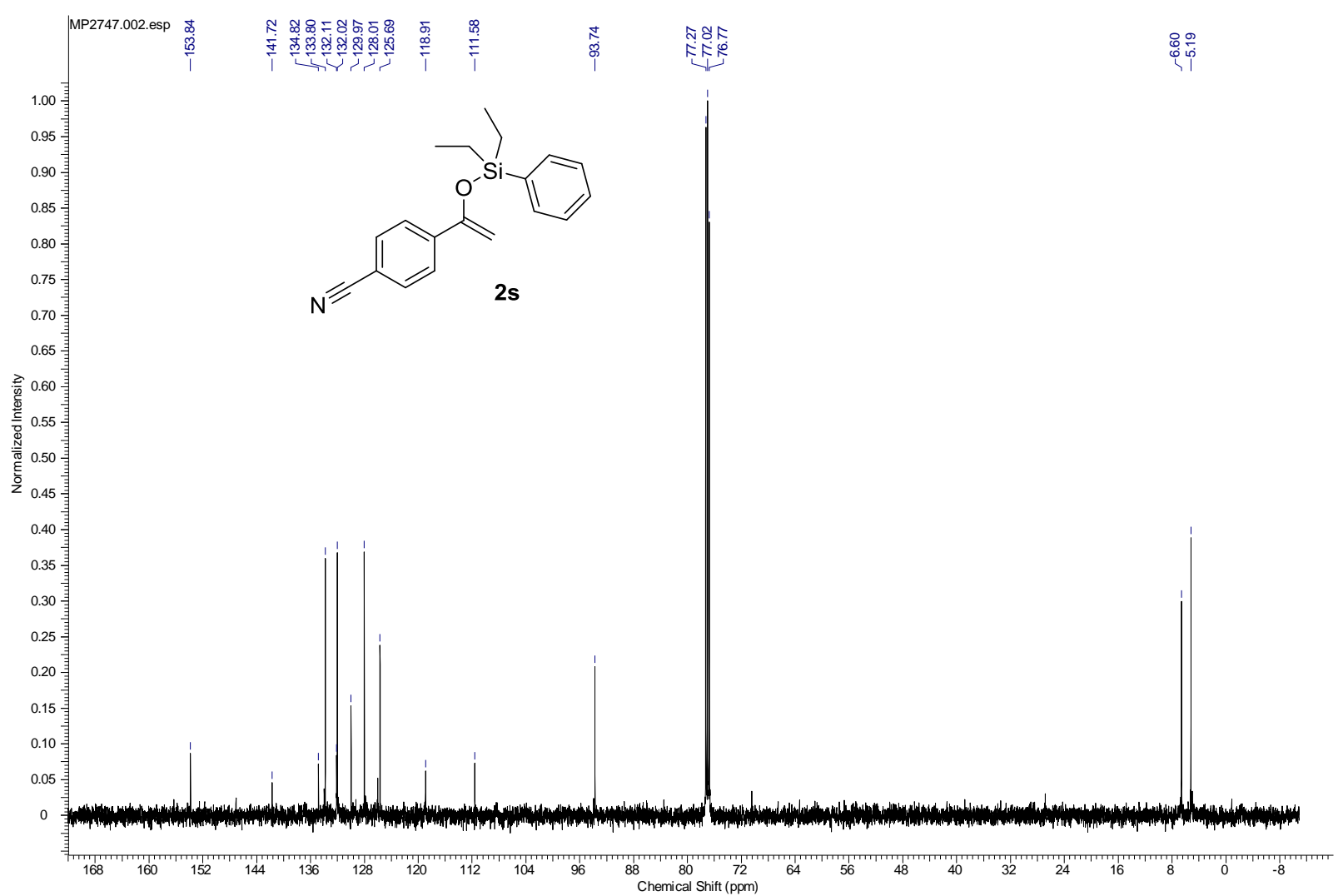




\section{${ }^{1} \mathrm{H}$ Spectrum of $\mathbf{2 t}$}

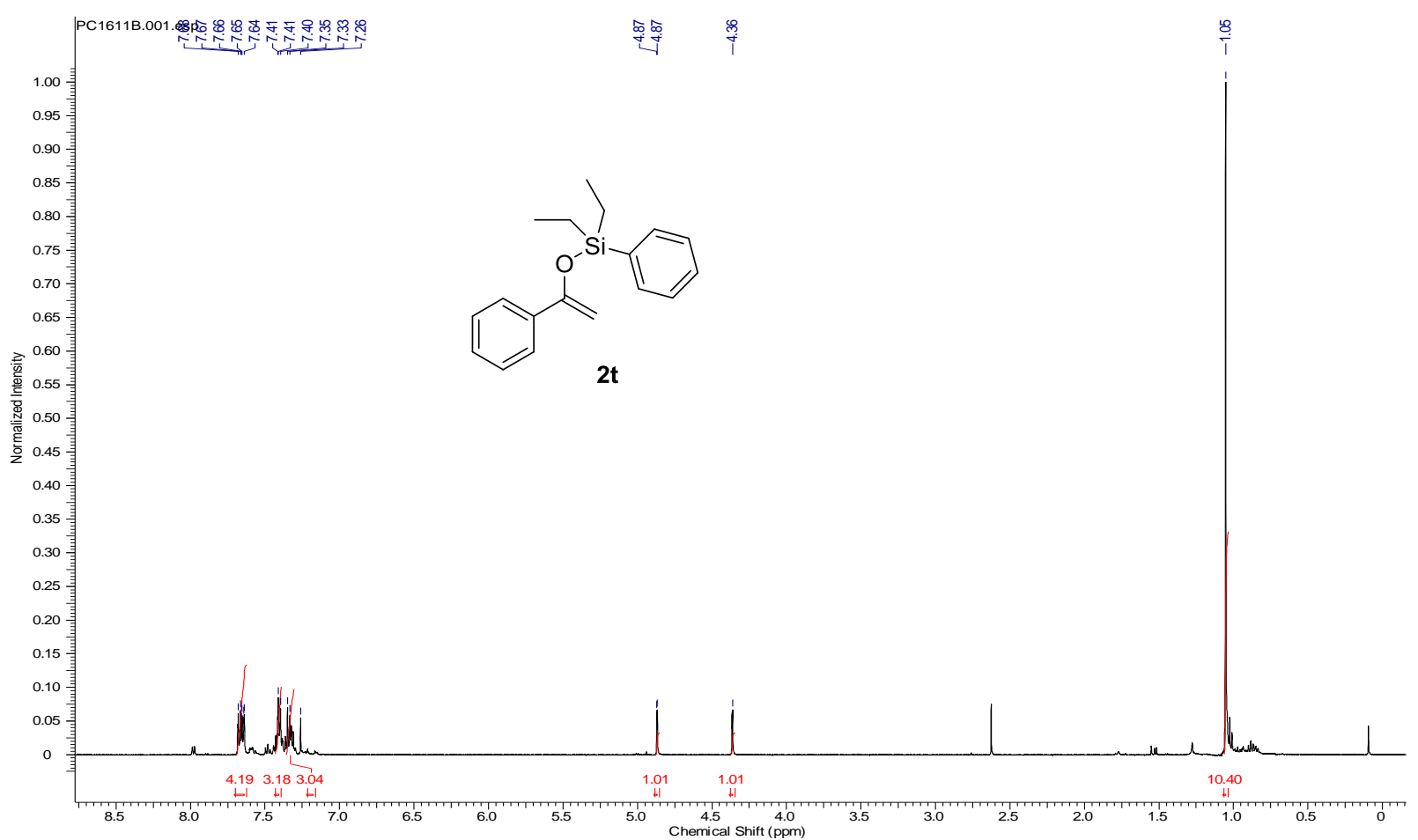

\section{${ }^{13} \mathrm{C}$ Spectrum of $\mathbf{2 t}$}

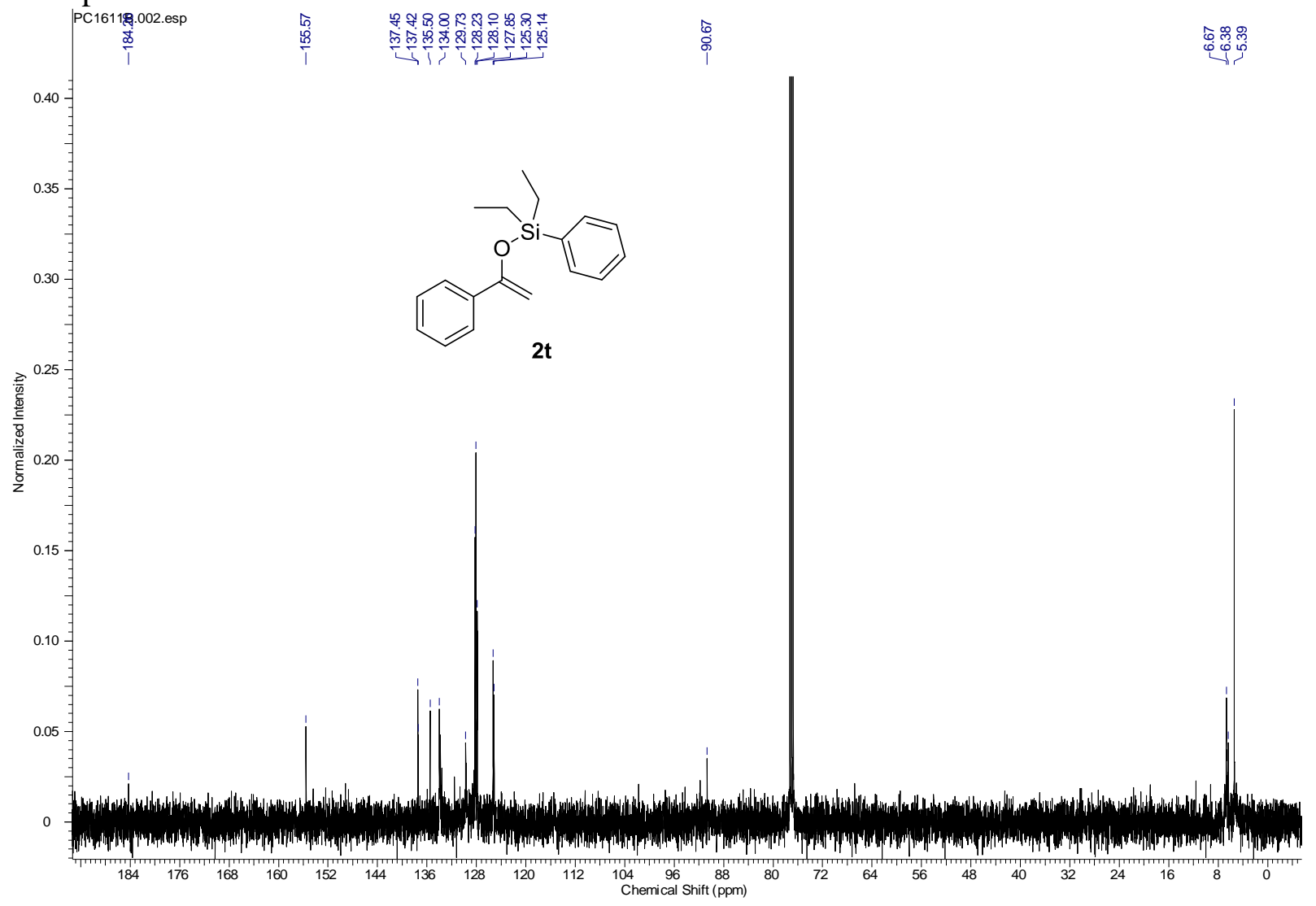




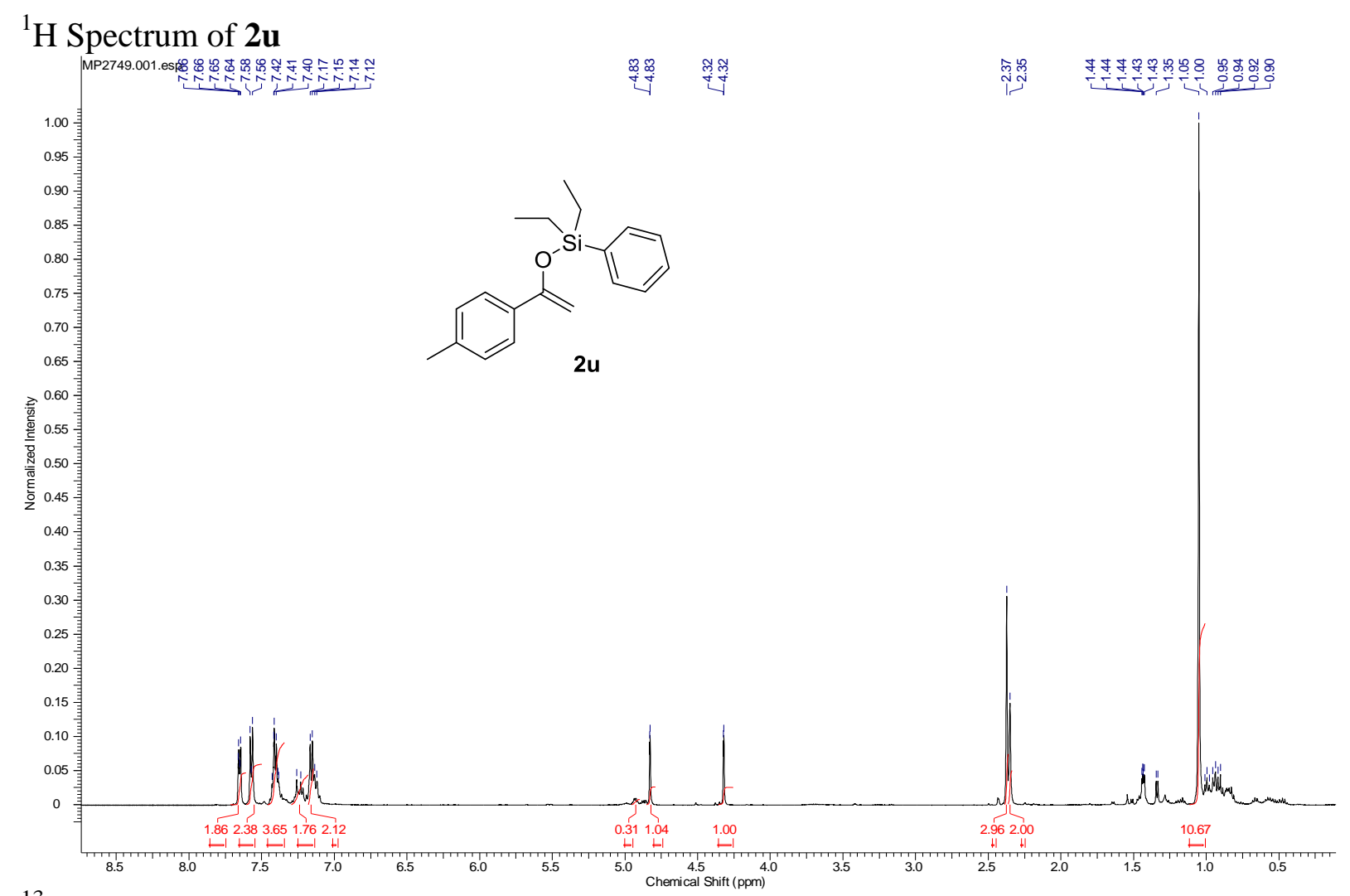

\section{${ }^{13} \mathrm{C}$ Spectrum of $\mathbf{2 u}$}

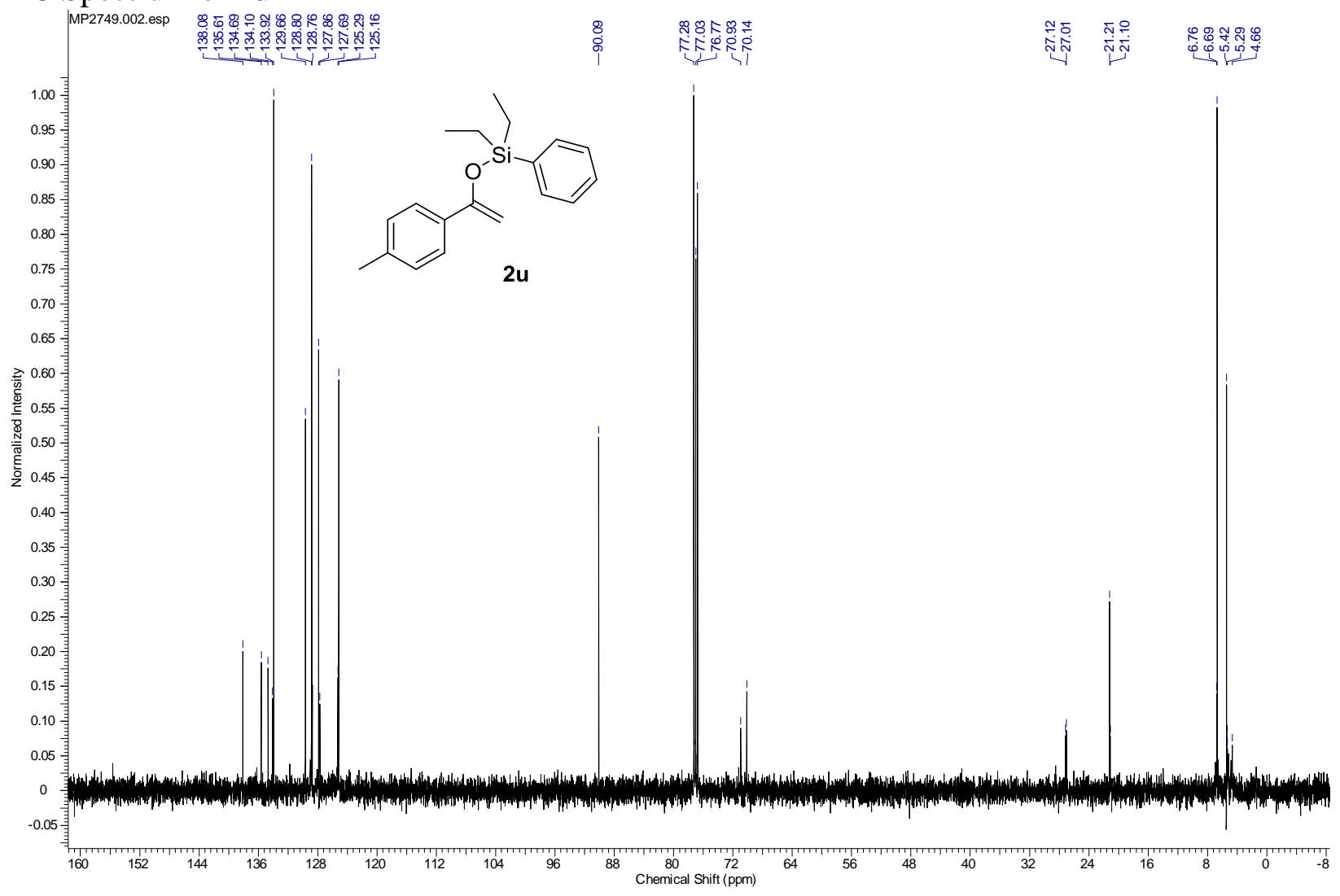




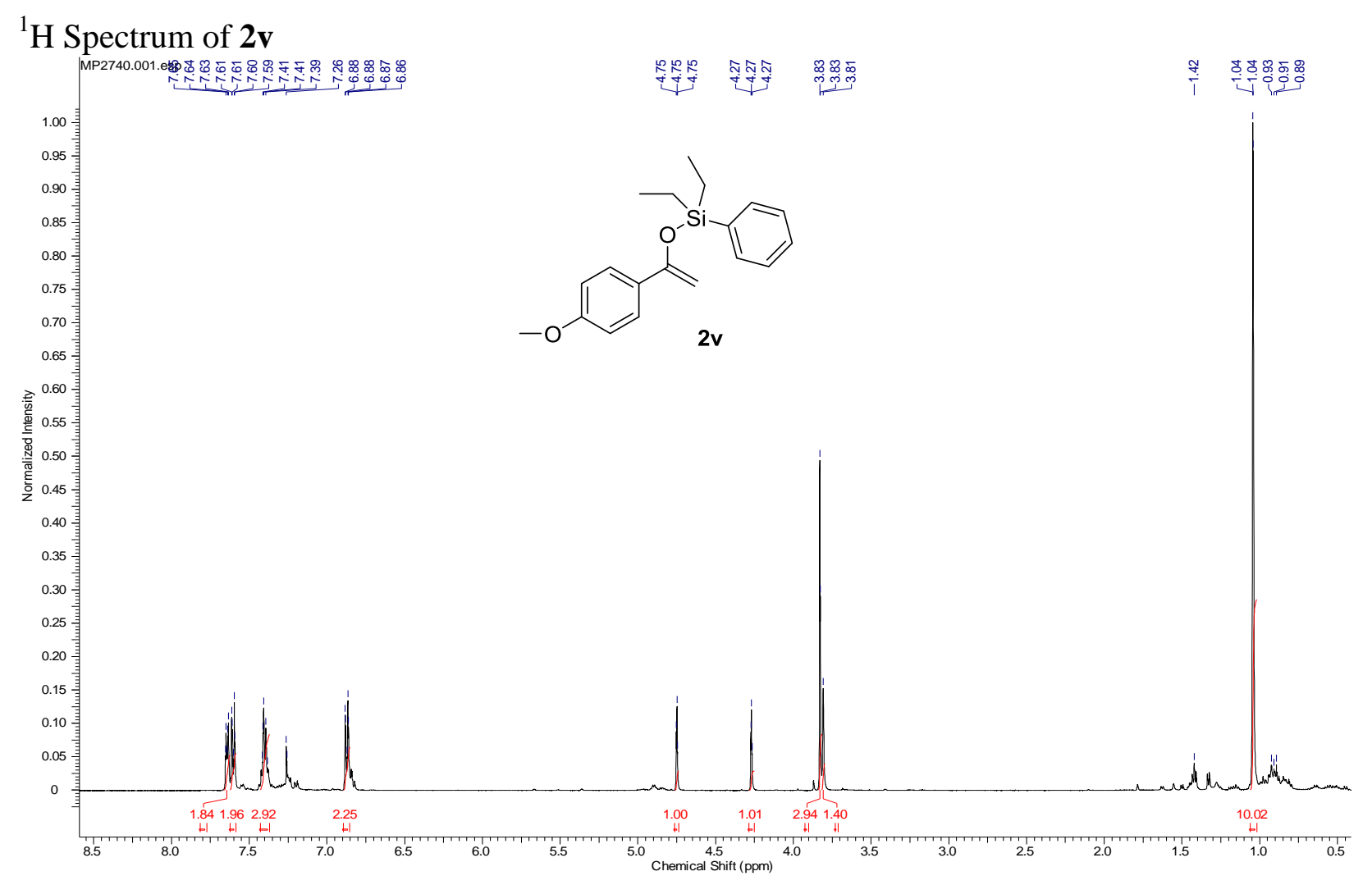

\section{${ }^{13} \mathrm{C}$ Spectrum of $\mathbf{2 v}$}

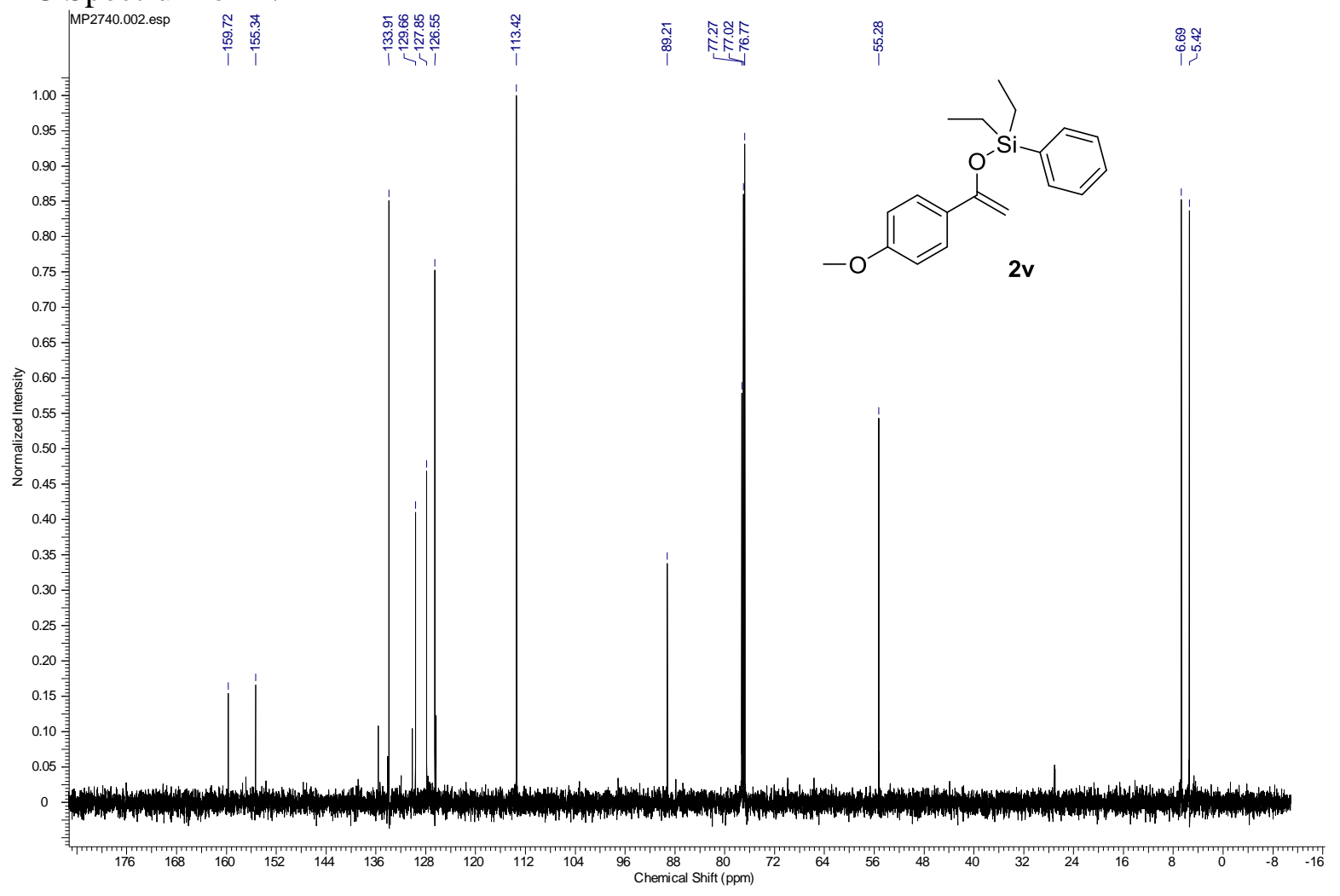



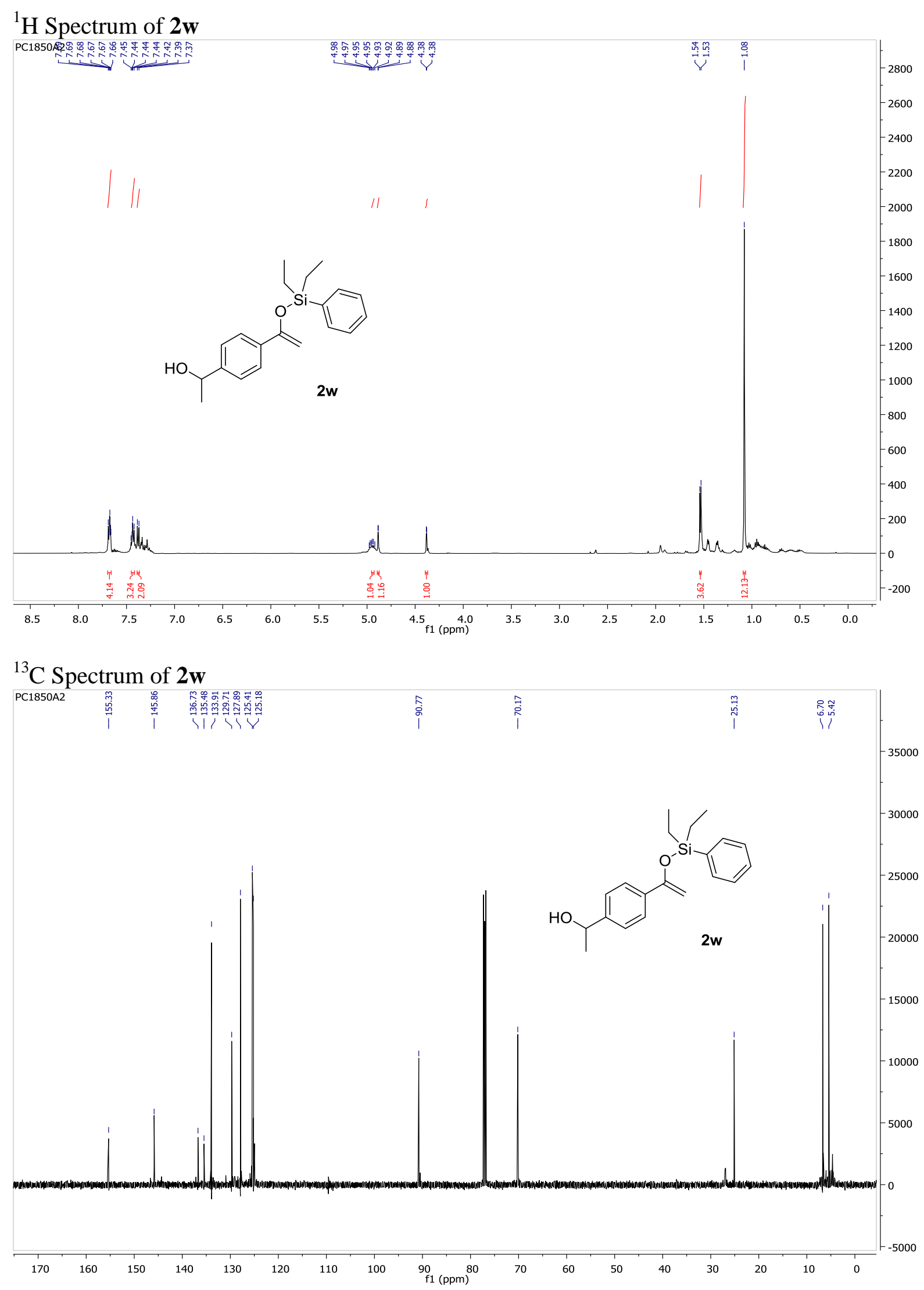

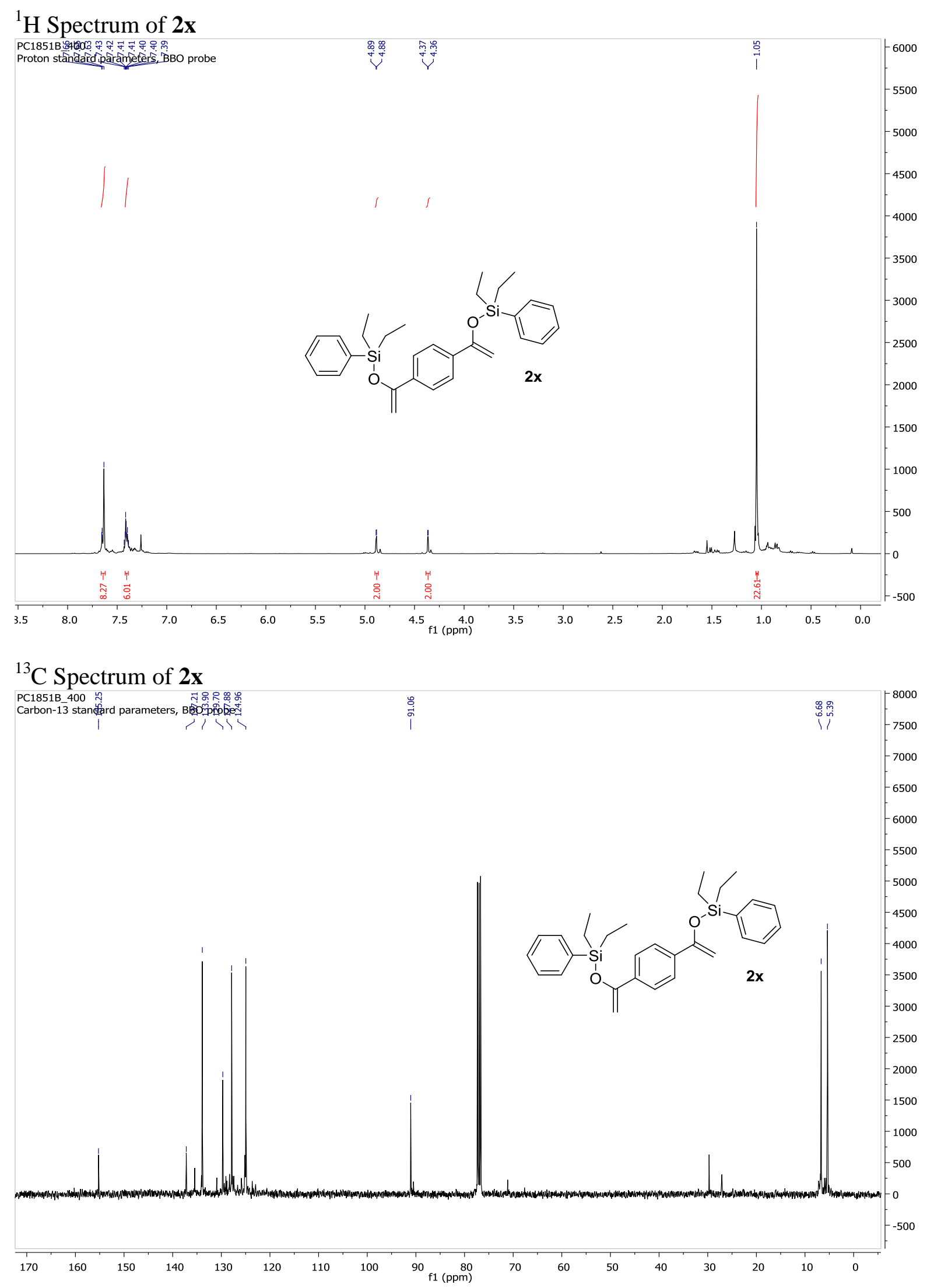


\section{${ }^{1} \mathrm{H}$ Spectrum of $\mathbf{2 y}, \mathbf{2 y}{ }^{\prime}$}

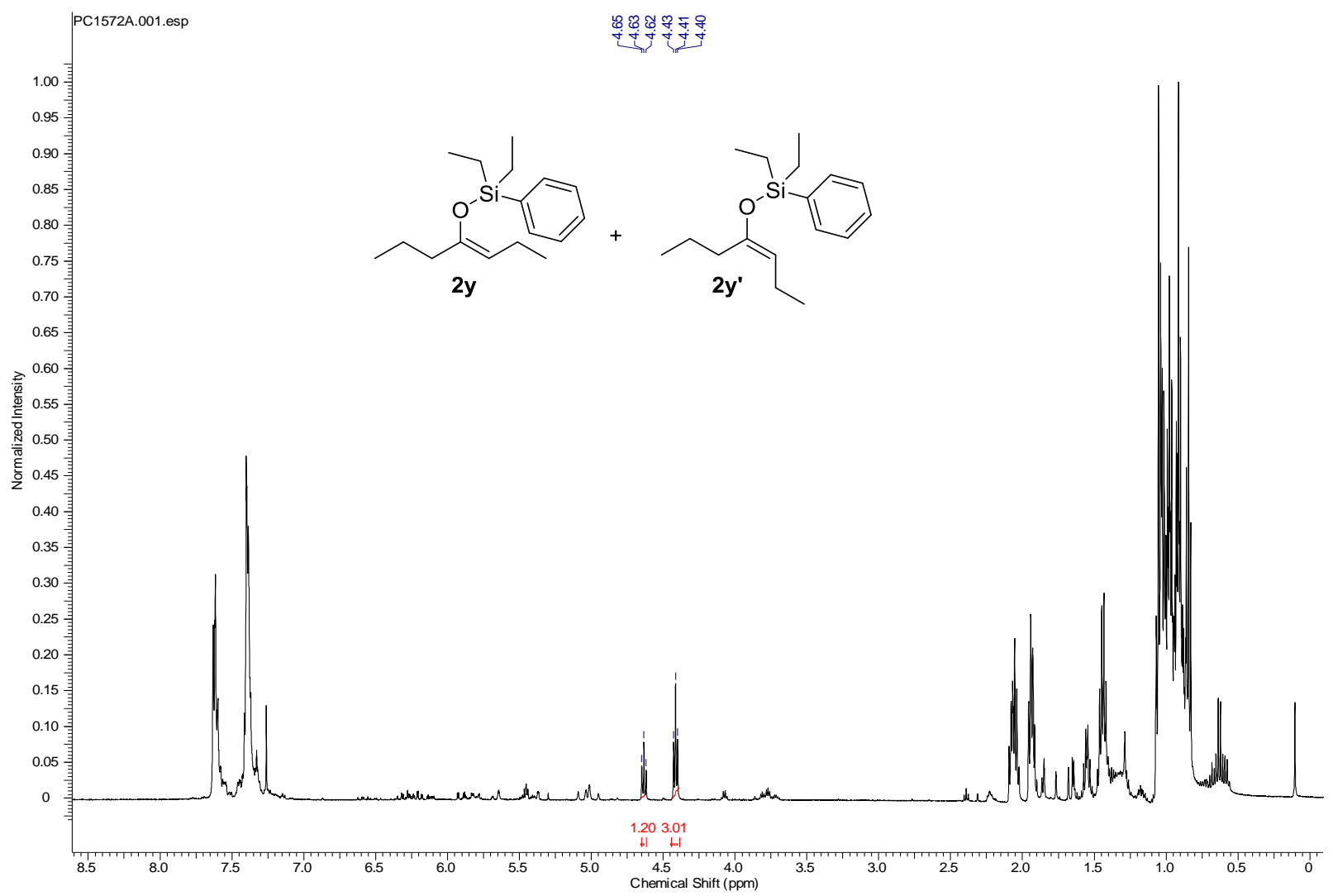

${ }^{13} \mathrm{C}$ Spectrum of $\mathbf{2 y}, \mathbf{2 y}$,

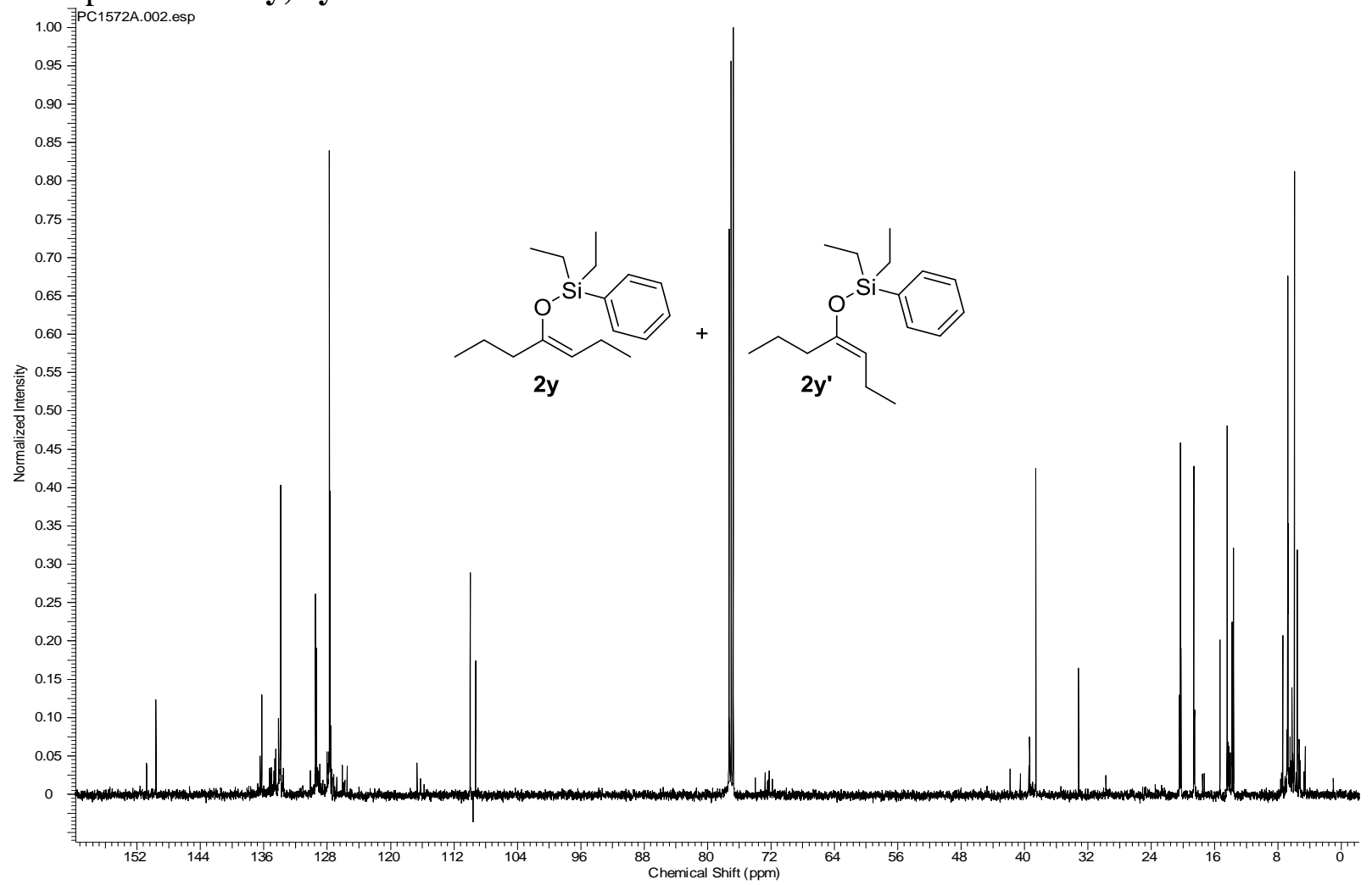




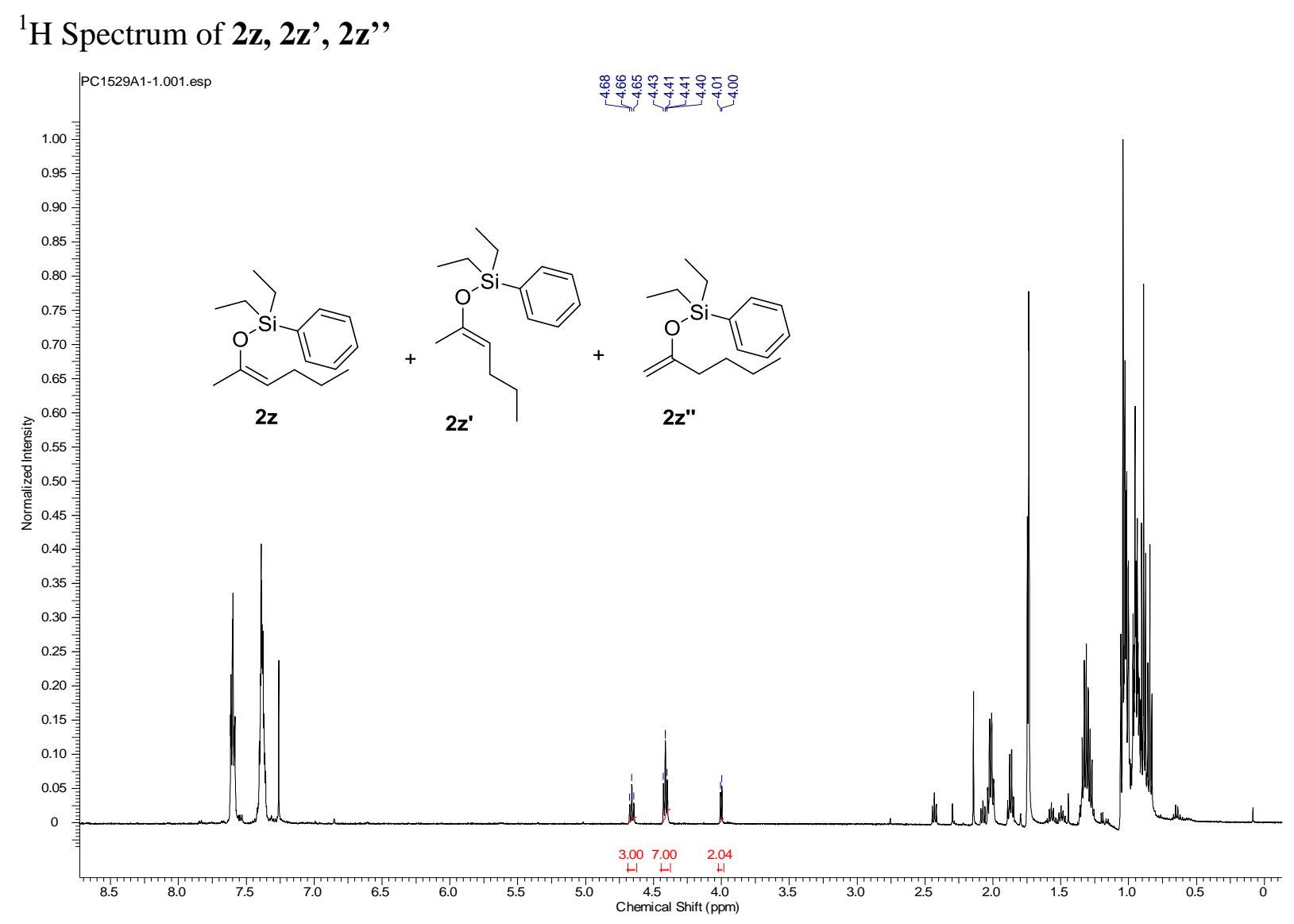

${ }^{13} \mathrm{C}$ Spectrum of $\mathbf{2 z}, \mathbf{2 z}, \mathbf{2 z}$,

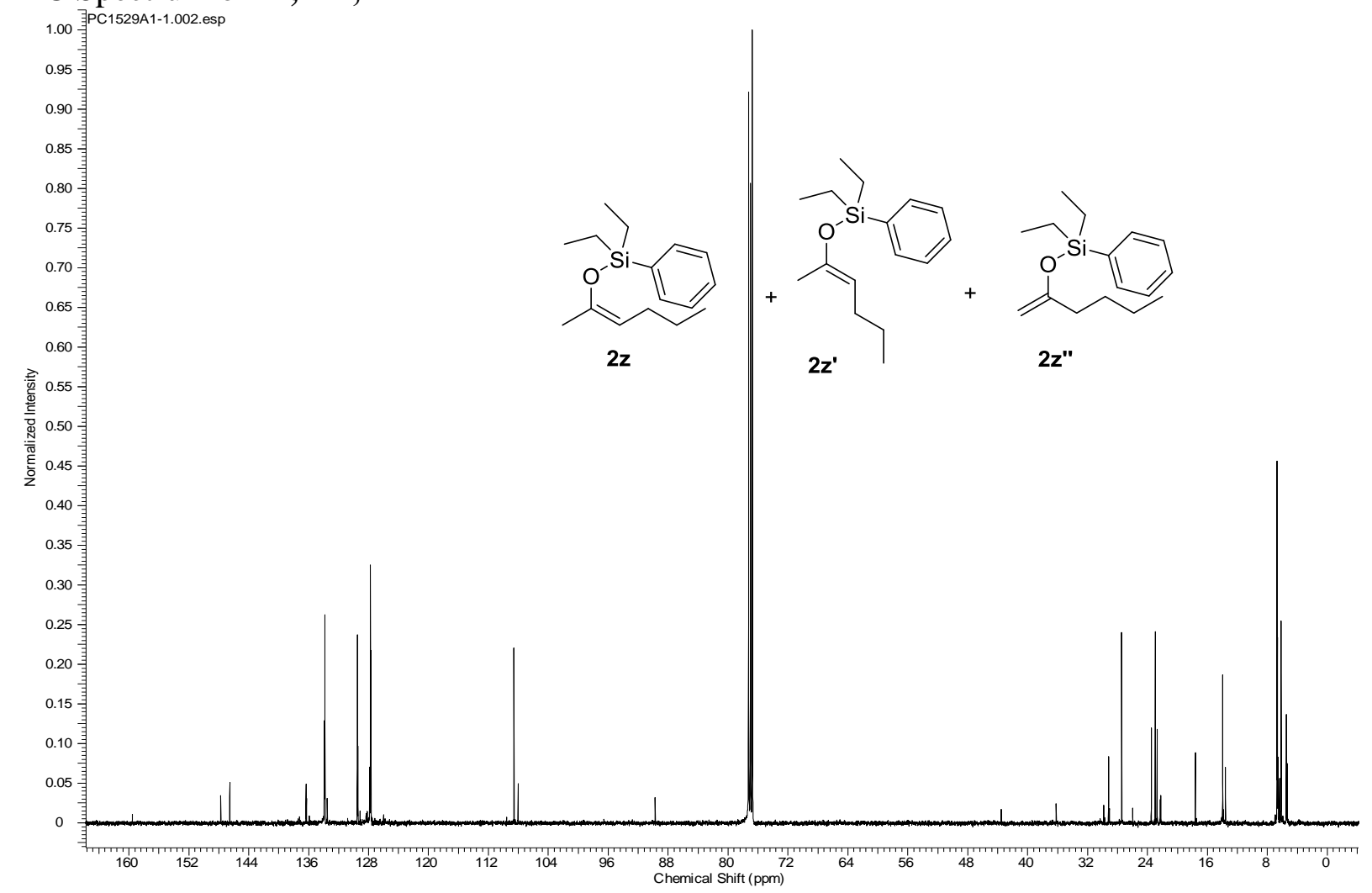




\section{${ }^{1} \mathrm{H}$ Spectrum of $\mathbf{2 a a}$}

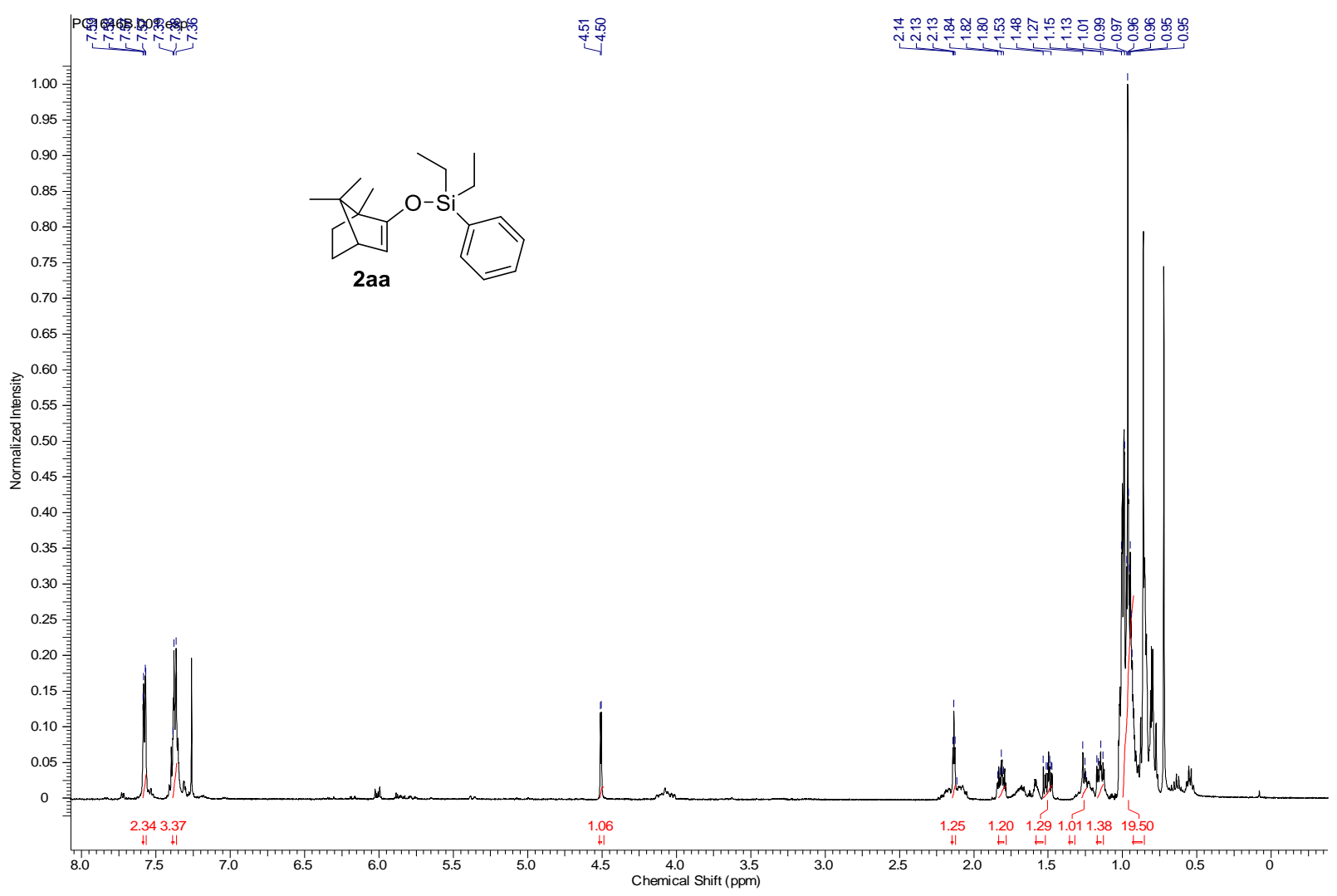

\section{${ }^{13} \mathrm{C}$ Spectrum of $\mathbf{2 a a}$}

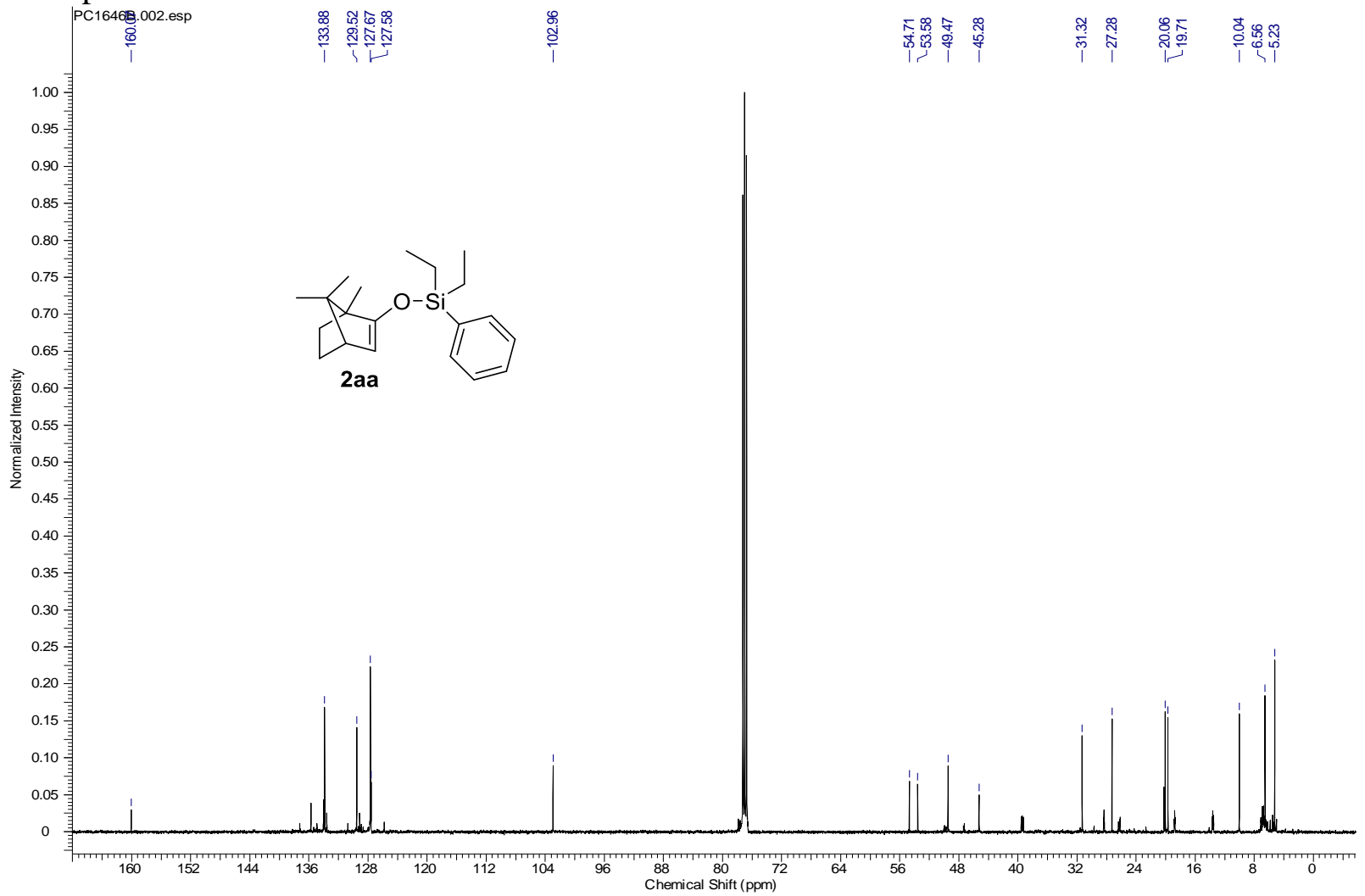



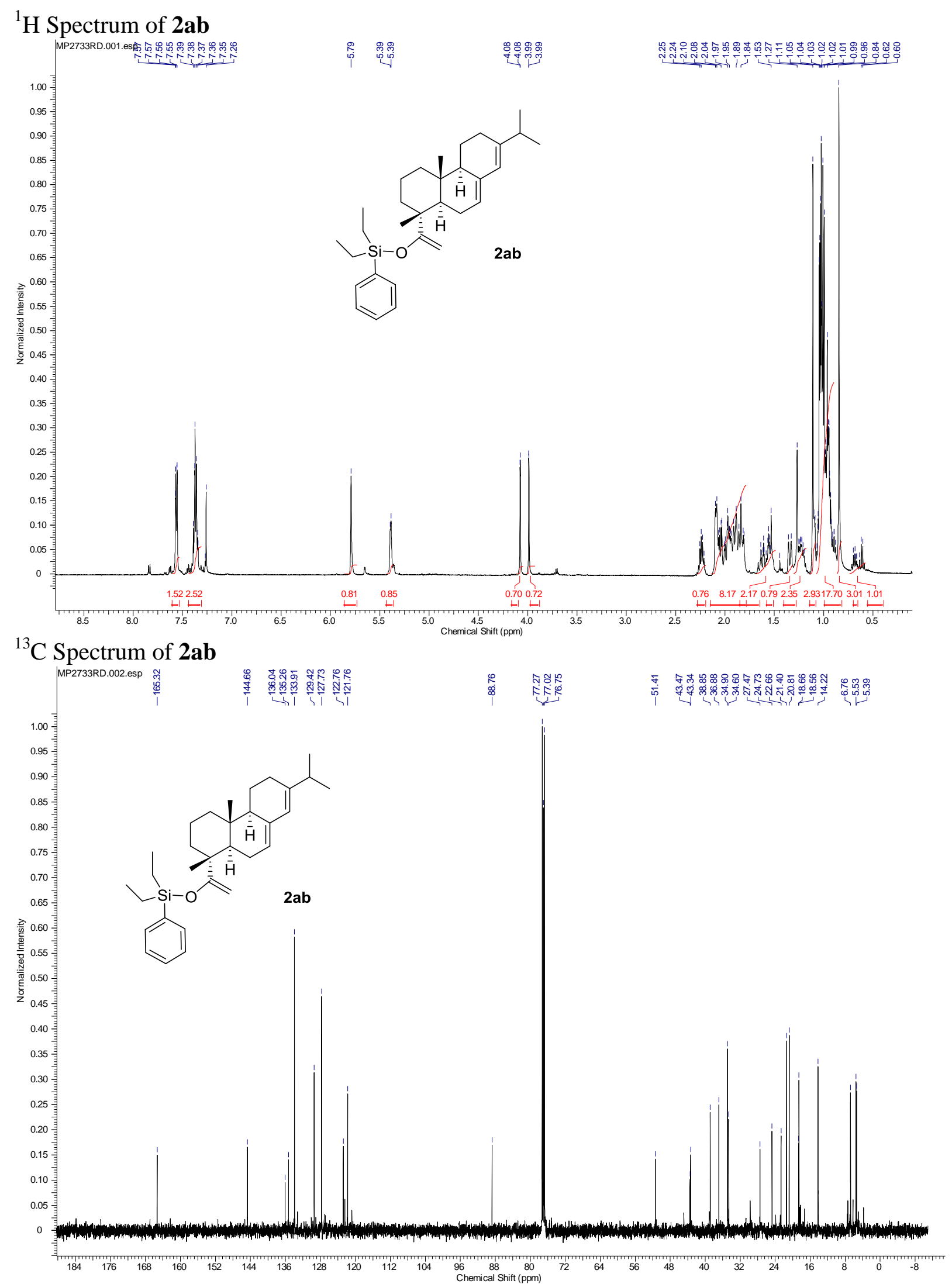


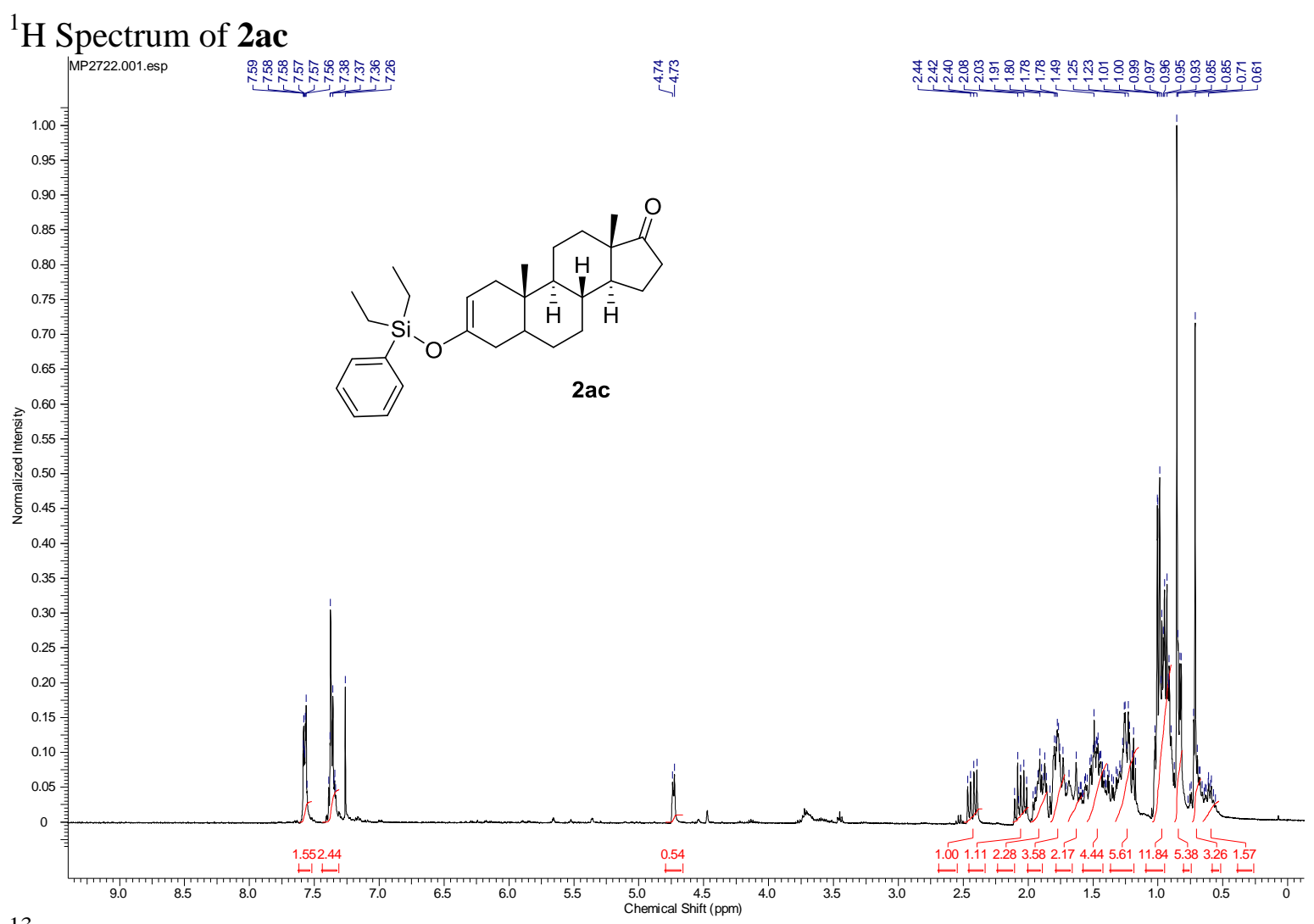

${ }^{13} \mathrm{C}$ Spectrum of $\mathbf{2 a c}$

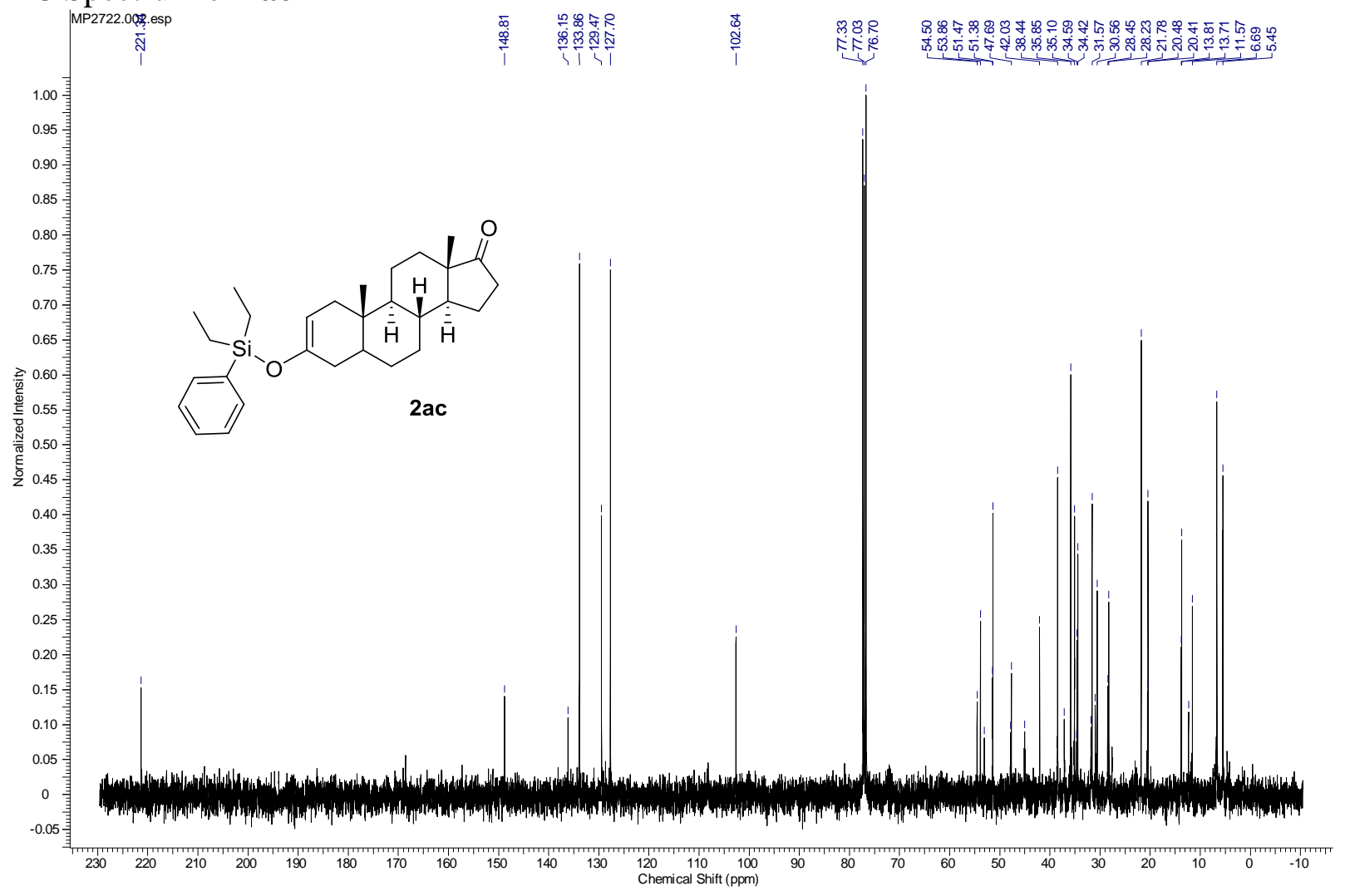




\section{${ }^{1} \mathrm{H}$ Spectrum of 1a-d}

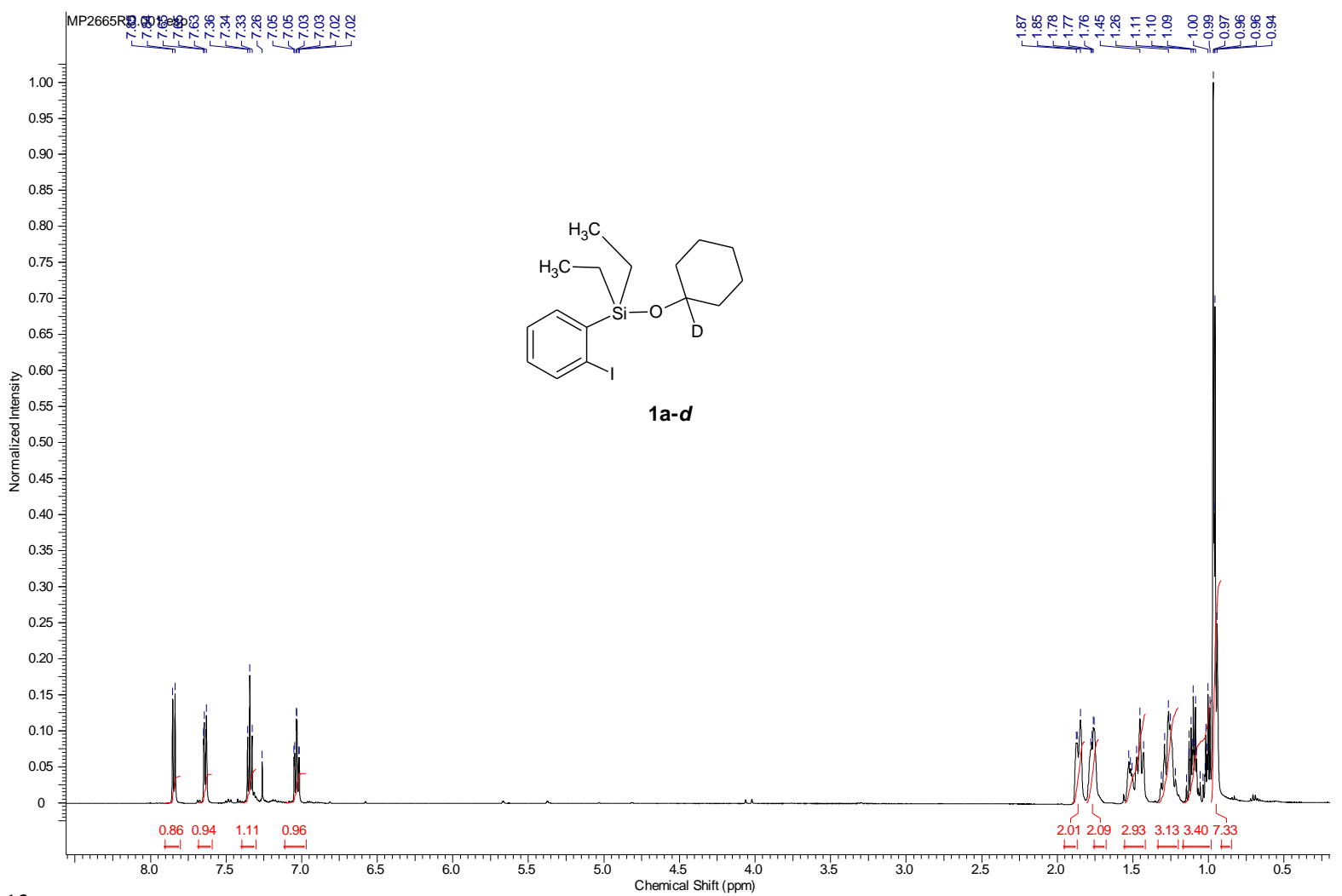

\section{${ }^{13} \mathrm{C}$ Spectrum of 1a-d}

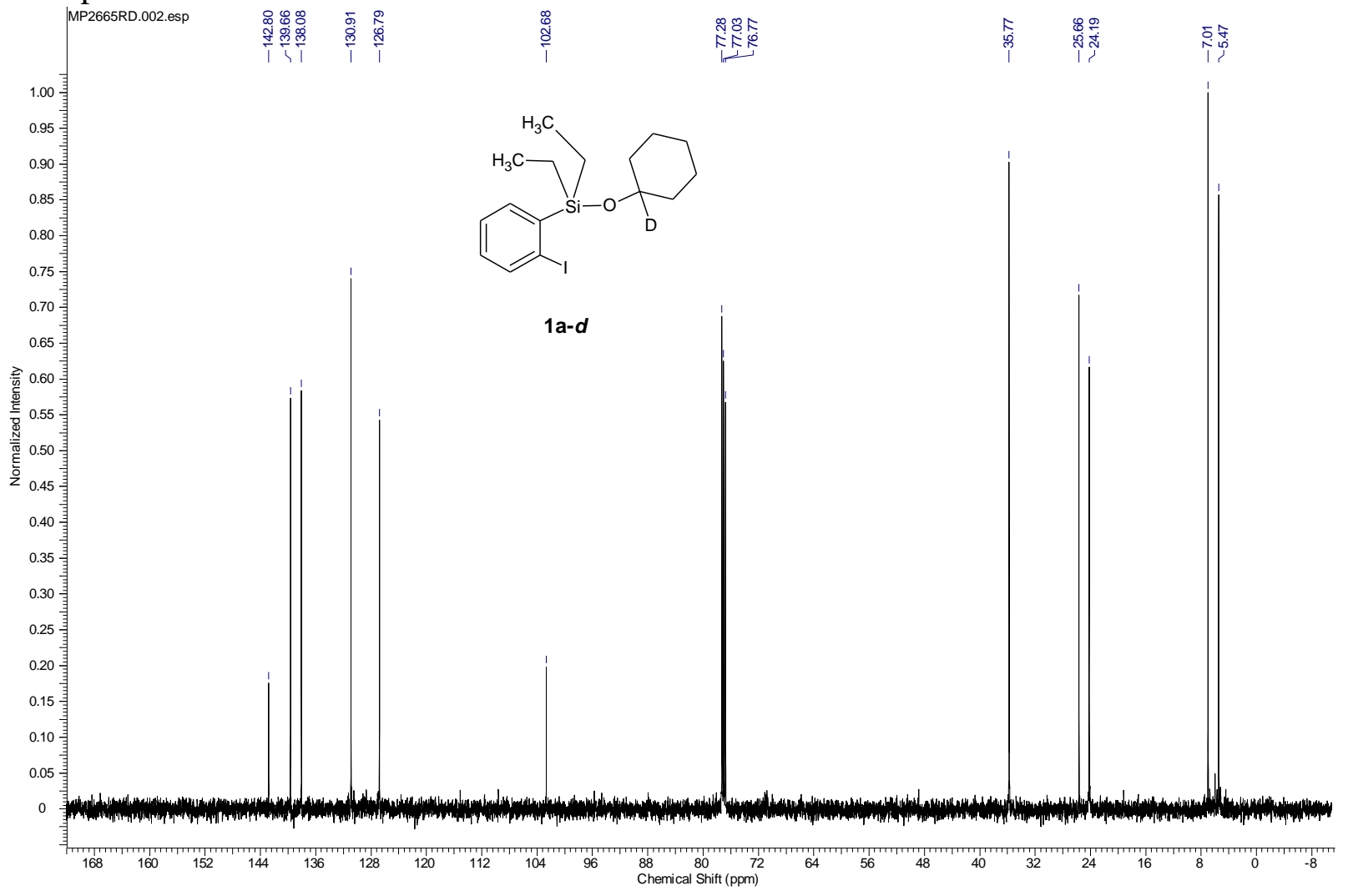




\section{${ }^{2} \mathrm{H}$ Spectrum of $\mathbf{1 a - d}$}

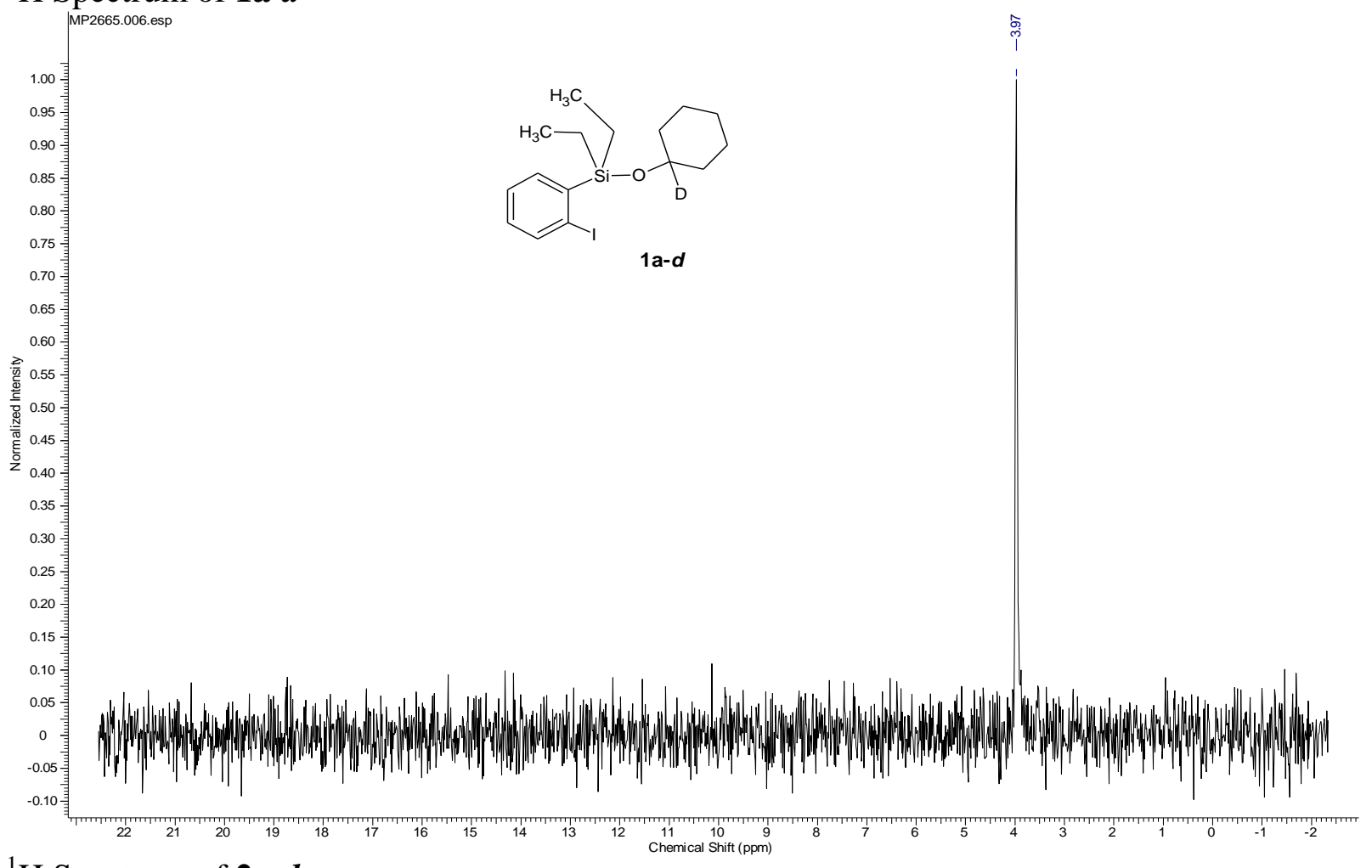

${ }^{1} \mathrm{H}$ Spectrum of $\mathbf{2 a -} \boldsymbol{d}$

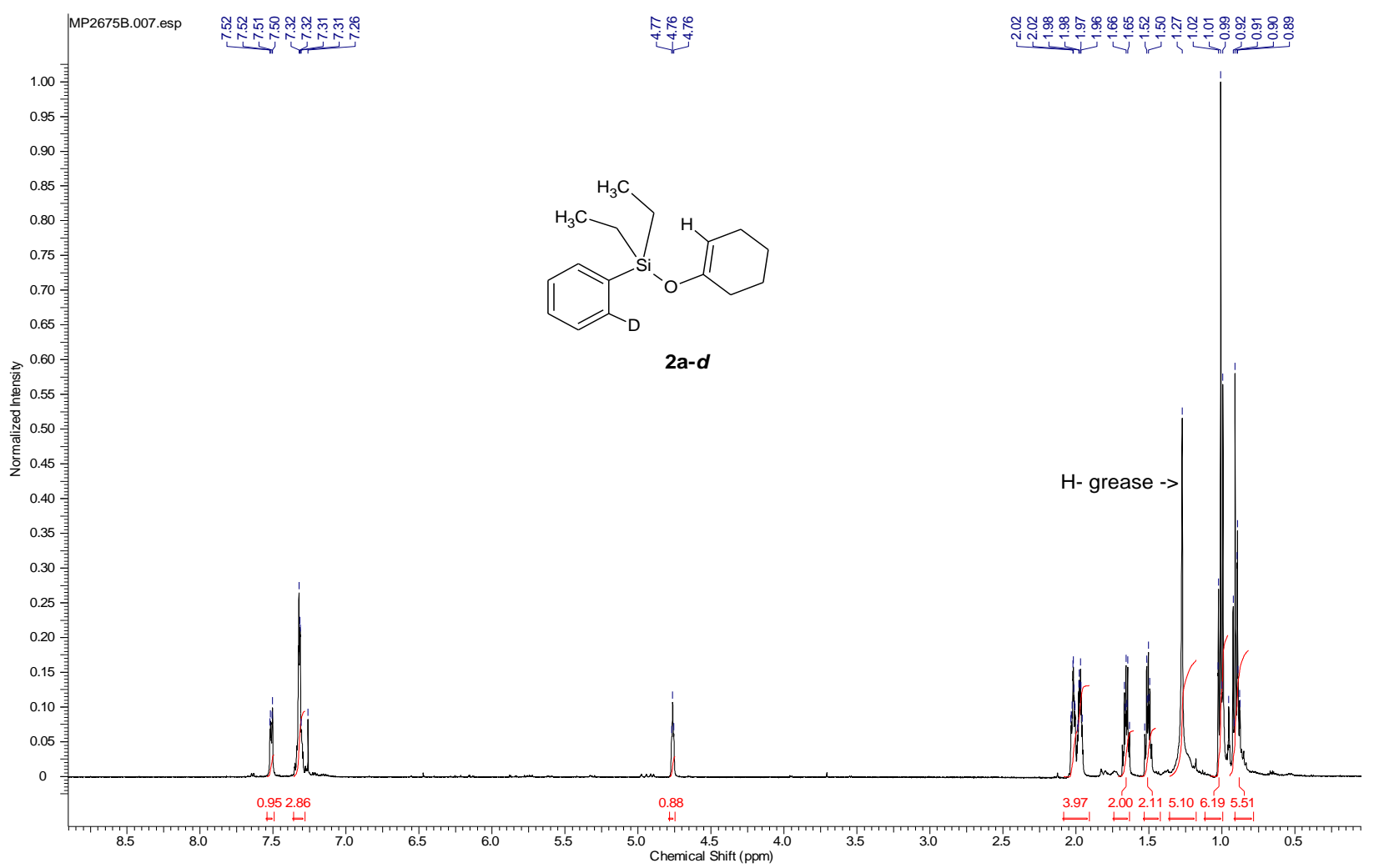




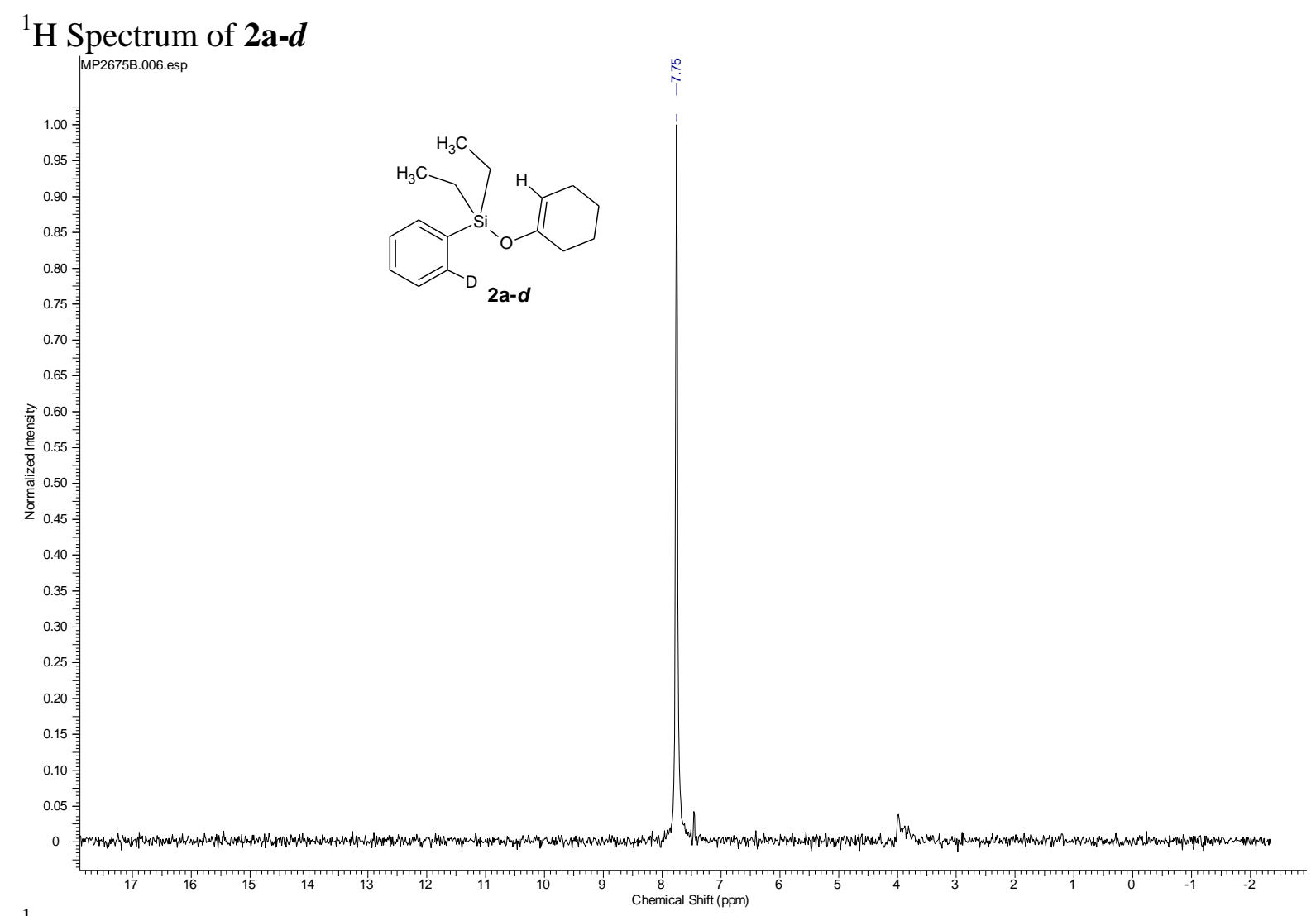

${ }^{1} \mathrm{H}$ Spectrum of $\mathbf{1 3}$

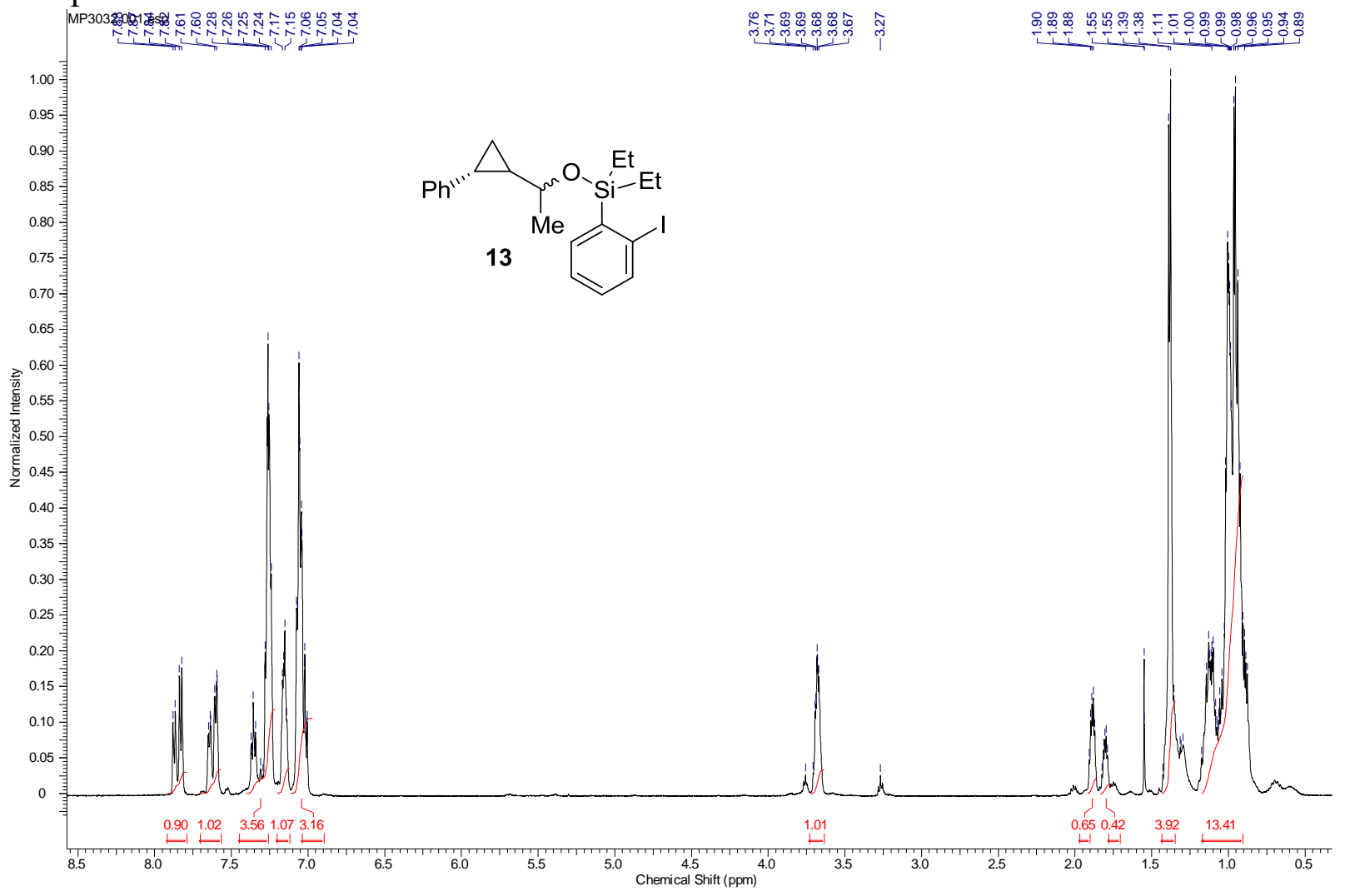




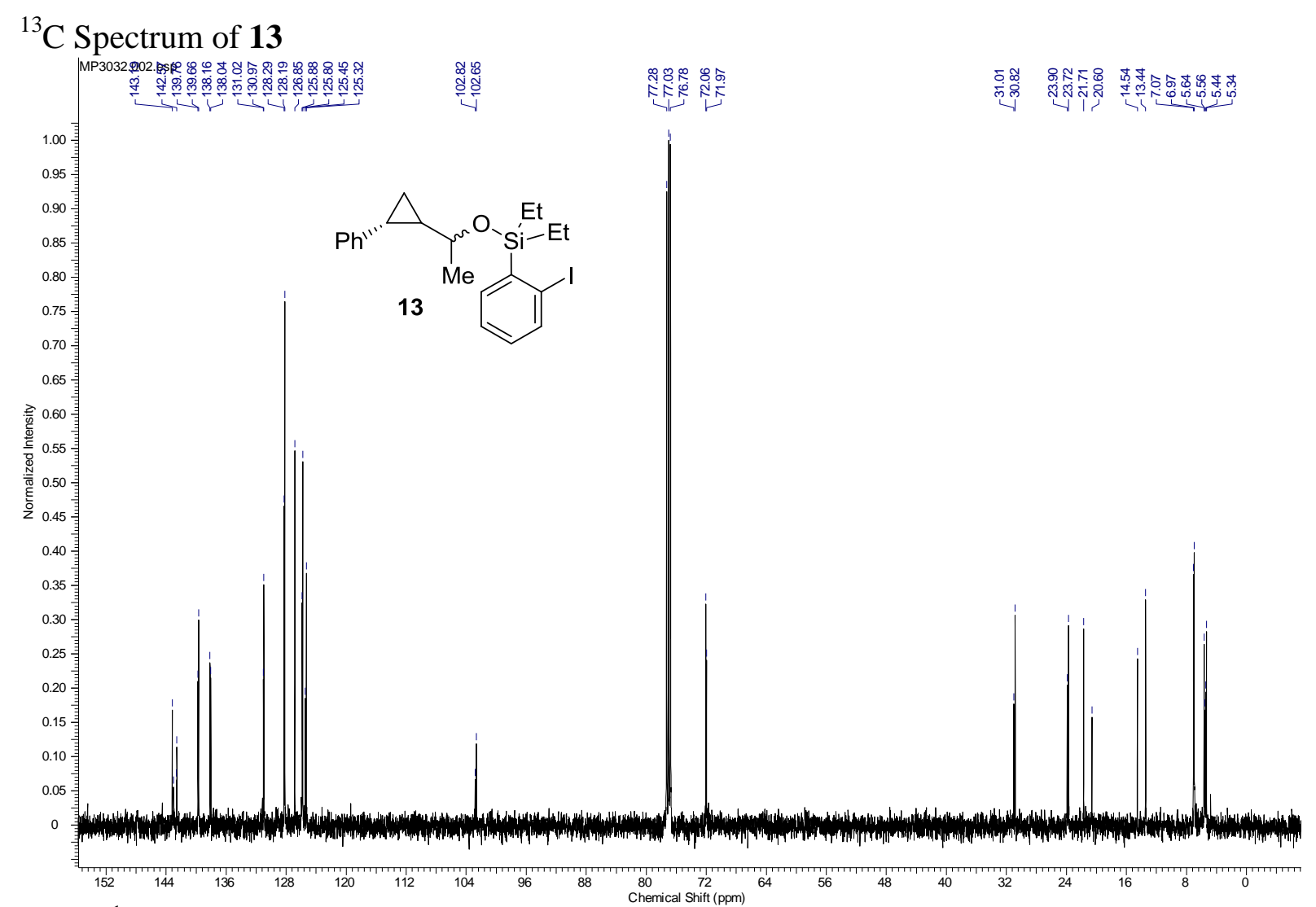

\section{Crude ${ }^{1} \mathrm{H}$ Spectrum of $\mathbf{1 6}$}

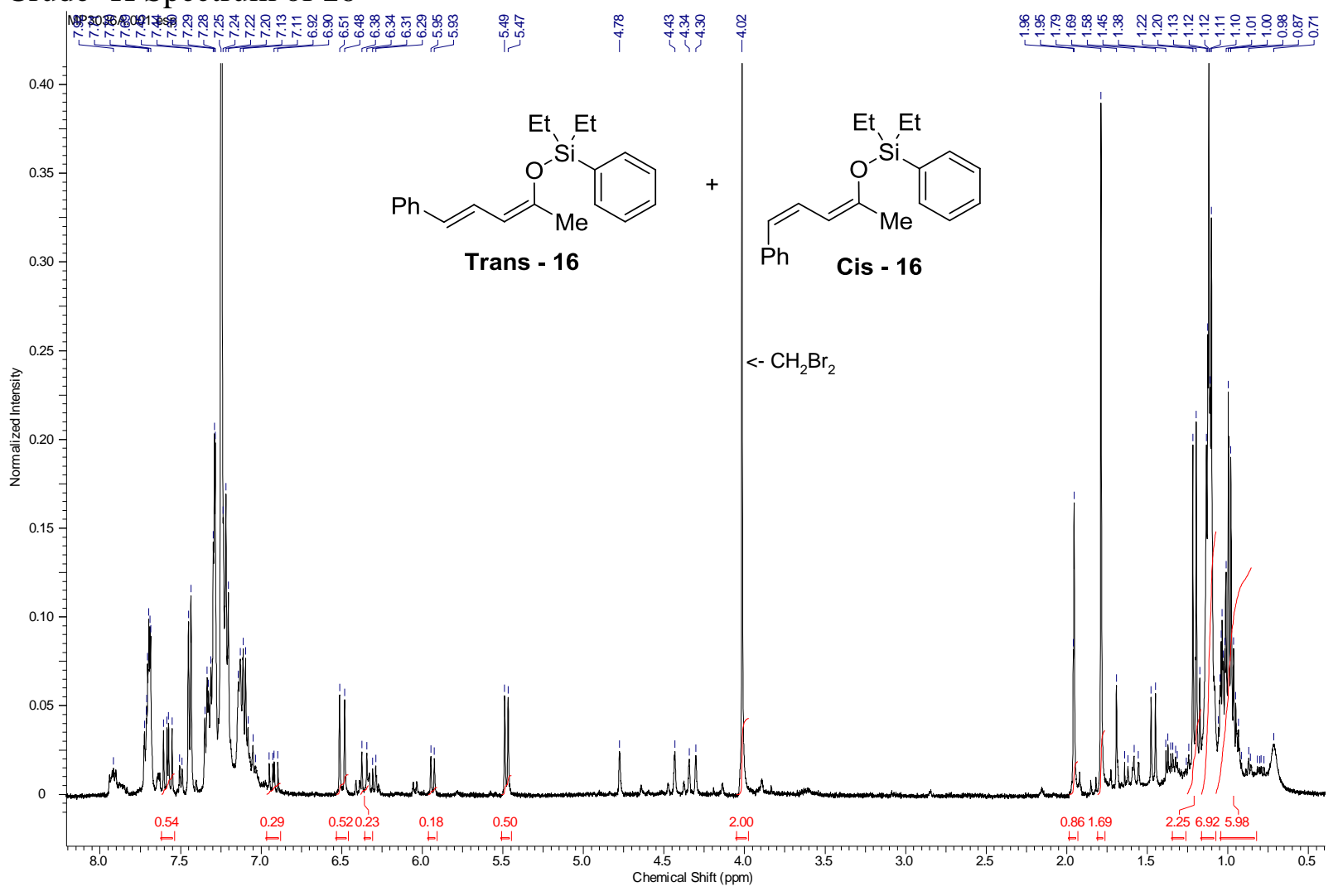




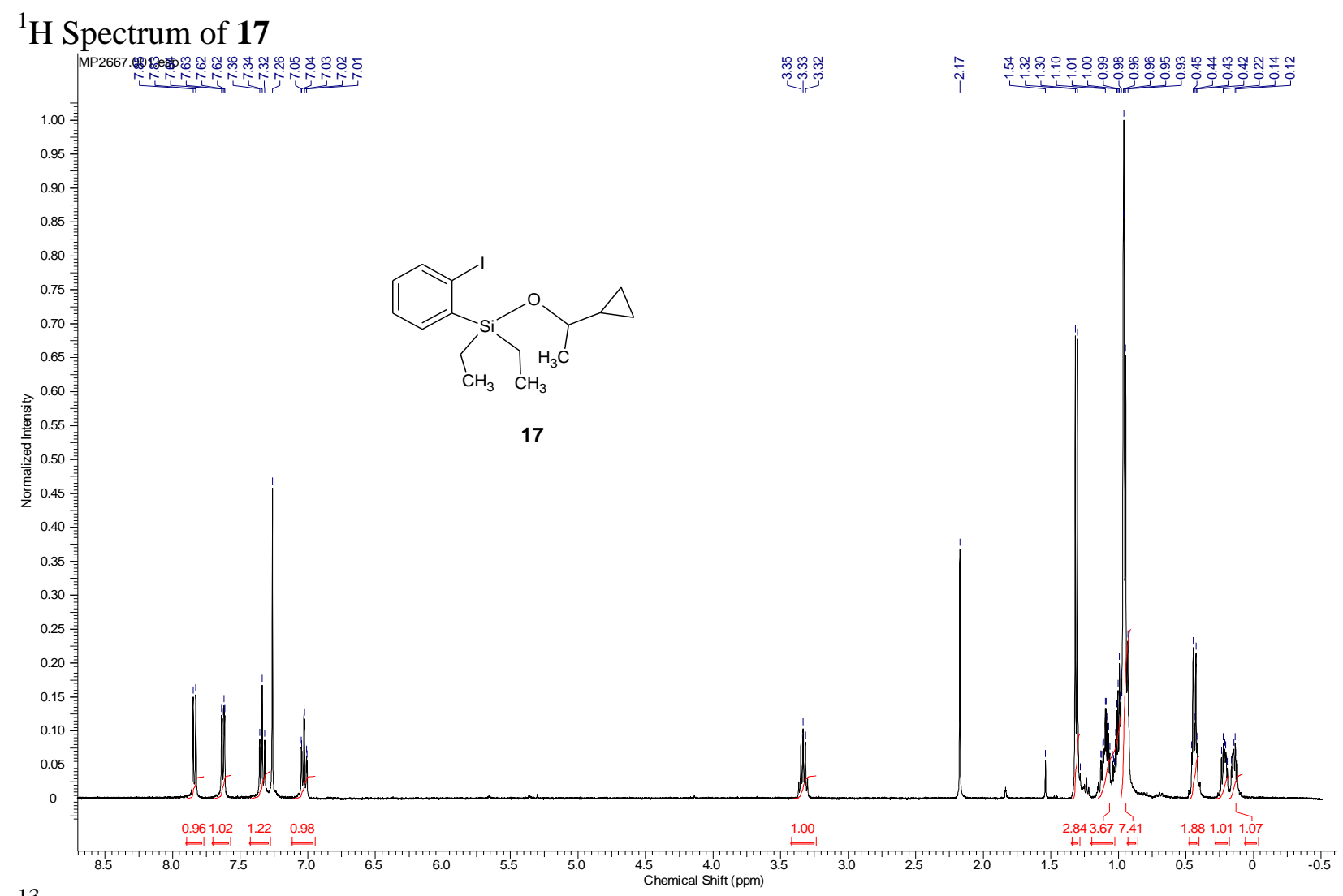

${ }^{13} \mathrm{C}$ Spectrum of $\mathbf{1 7}$

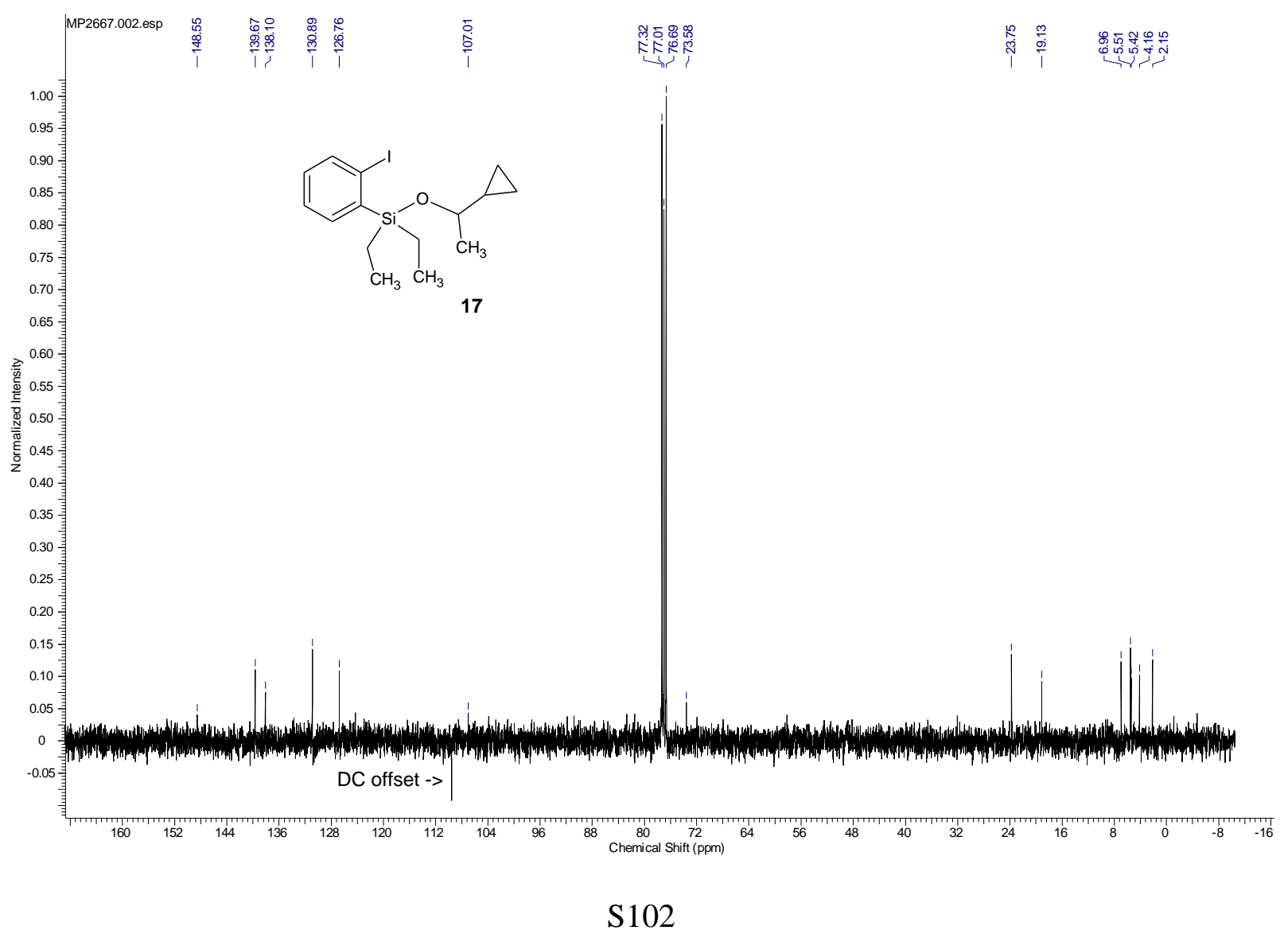




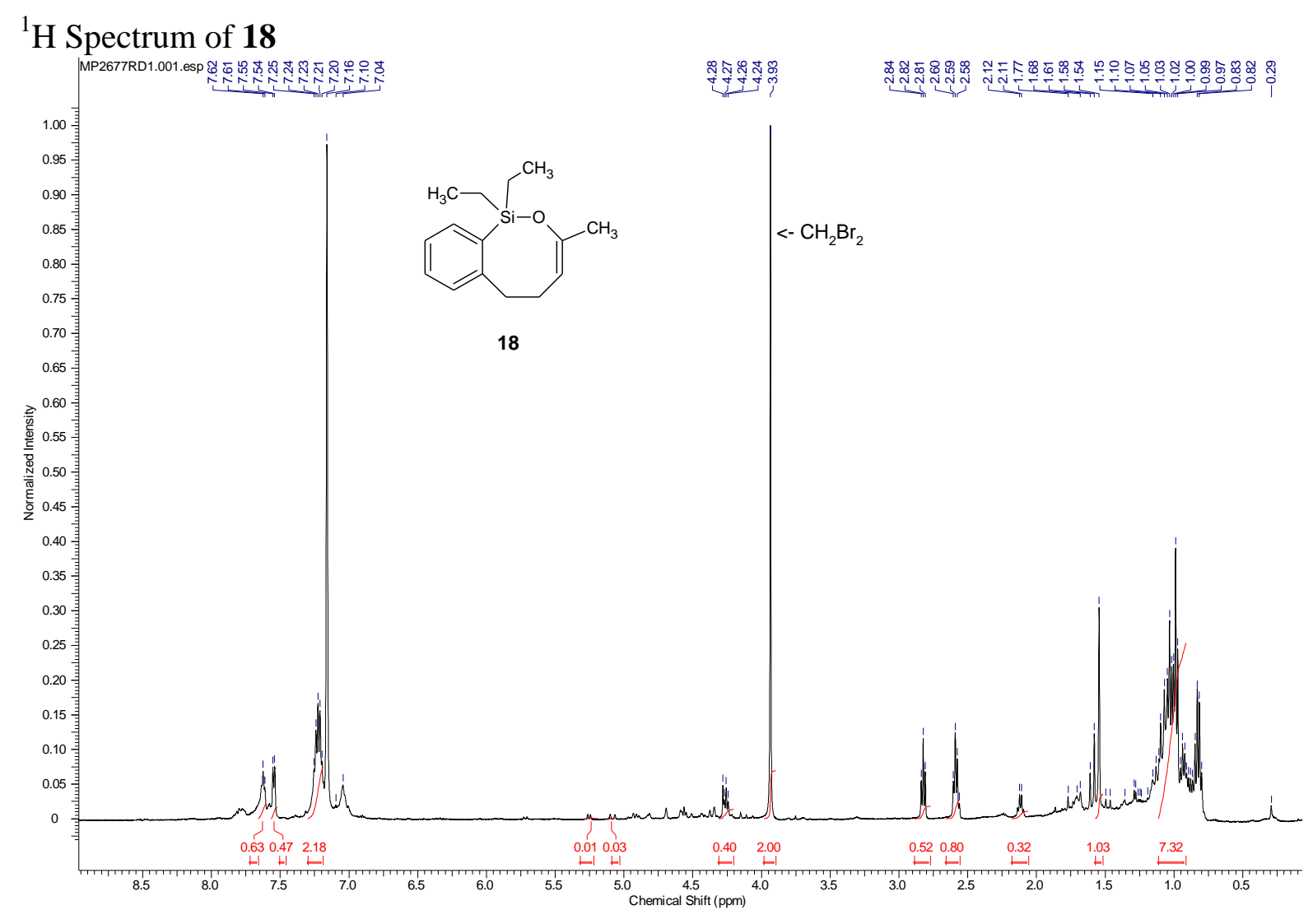




\section{References}

(1) González, M. A.; Correa-Royero, J.; Agudelo, L.; Mesa, A.; Betancur-Galvis, L. Eur. J. Med. Chem. 2009, 44, 2468.

(2) Curran, D. P.; Kim, D.; Liu, H. T.; Shen, W. J. Am. Chem. Soc. 1988, 110, 5900.

(3) Bag, S.; Patra, T.; Modak, A.; Deb, A.; Maity, S.; Dutta, U.; Dey, A.; Kancherla, R.; Maji, A.; Hazra, A.; Bera, M.; Maiti, D. J. Am. Chem. Soc. 2015 137, 11888.

(4) Kakei, H.; Sone, T.; Sohtome, Y.; Matsunaga, S.; Shibasaki, M. J. Am. Chem. Soc. 2007, 129, 13410. 